Universidade de São Paulo

Instituto de Astronomia, Geofísica e Ciências Atmosféricas

Departamento de Astronomia

Diana Renata Gonçalves Gama

Formação Estelar no Complexo de Nuvens

Moleculares em Monoceros

São Paulo

2012 

Diana Renata Gonçalves Gama

\section{Formação Estelar no Complexo de Nuvens Moleculares em Monoceros}

Dissertação apresentada ao Departamento de Astronomia do Instituto de Astronomia, Geofísica e Ciências Atmosféricas da Universidade de São Paulo como requisito parcial para a obtenção do título de Mestre em Ciências.

Versão corrigida. O original encontra-se disponível na Unidade.

Área de Concentração: Astronomia Orientadora: Prof. ${ }^{a}$ Dr. ${ }^{a}$ Jane Gregorio-Hetem

São Paulo 

Dedico esse trabalho aos meus pais, que tornaram possíveis meus passos, desde os primeiros. 



\section{Agradecimentos}

Grande parte desse trabalho não seria possível - ou seria certamente mais difícil - sem a presença de algumas pessoas nessa etapa tão importante da minha vida. Se por um lado falta espaço para todos os nomes, por outro lado sobra gratidão e apreço.

Primeiramente, agradeço à minha família: meus pais Mercedes e Dimas que foram o meu porto seguro (mesmo com a distância), sempre esperando pela minha próxima decisão, sempre sentindo orgulho do caminho que escolhi, sempre mantendo firme o propósito de me educar e ensinar a valorizar cada conquista em minha vida; às minhas irmãs Daniela e Debora que foram exemplos de força de vontade e resistência, exemplos de que alcançar um objetivo não é fácil, porém não é impossível quando se quer de verdade; aos meus padrinhos Glória e Gilberto que acreditaram no meu sonho e no meu potencial, me apoiando sempre e como podiam, mostrando o real significado da responsabilidade que lhes foi concedida; a todos que me proporcionaram momentos divertidos, palavras de motivação, companheirismo incomparável, que me deram motivos para nunca me esquecer de onde eu vim - em especial ao meu primo Yuri que, com entusiasmo e fascinação, me ensinou a olhar as estrelas e nelas encontrar inspiração, sem imaginar que anos depois essa ainda seria a paixão que me move.

Aos meus amigos, de longe e de perto: Luciana Caminha que sempre me acompanhou e incentivou; Elisa Zamat que sempre teve um cantinho no coração guardado só para mim; Thiago Lopes que sempre me fez rir e pensar; Rafael Everton que nunca me deixou desistir e sempre me ouviu com atenção e interesse; Aline Durães que sempre me ofereceu sinceridade e apoio; aos querido Osvaldo Souza, Gabriel Barros e Jeferson Felix que acompanharam em grande parte da minha vida acadêmica, companheiros também de observações em Valinhos e de aventuras no grupo Sputnik; Thiago Monfredini com quem 
desenvolvi uma amizade verdadeira, que foi além das observações no $R O I$ (e além dos masers!) e Fran Lima com quem aprendi muito sobre amizade, convivência, desabafos, cafés e $\mathrm{HT}_{\mathrm{E} X}$. Agradeço às pessoas que me apoiaram e me ajudaram a chegar onde eu estou, e agradeço às que não apoiaram também, pois certamente aproveitei essa chance para me tornar uma pessoa mais forte.

À Jane devo a maior parte dos agradecimentos desse trabalho, por me orientar ao longo desses anos, por dar vida à nossa pesquisa, por acreditar em meu potencial, por tornar real um sonho.

Ao meu colaborador Williams que abriu meus horizontes, deu crédito às minhas palavras, me iniciou na Rádio Astronomia, possibilitou novos caminhos e me desafiou com questionamentos que só me ajudaram a crescer.

Ao meu relator Antônio Mário Magalhães pelas sugestões relevantes e críticas construtivas.

Aos professores que fizeram parte da minha formação.

Aos colegas do Departamento de Astronomia da USP, que em algum momento me auxiliaram na pesquisa, na redação dessa dissertação, na discussão de assuntos relevantes e/ou contribuíram para um ambiente de trabalho diferenciado: Rodrigo Vieira, Marcus Duarte, Bruno Dias, Mairan Teodoro, Bruno Quint, Vinícius Placco, Oscar Cavichia, Felipe Navarete, Tiago Ricci, Grzegorz Kowal, Fellipy Silva, Pedro Beaklini, Paulo Lago, Carlos Braga e Gustavo Silva.

Ao pessoal da Secretaria do Departamento, Secretaria da Pós-Graduação e funcionários do IAG que me auxiliaram no que puderam e executaram seu trabalho de forma muito competente.

À CNPq, pelo apoio financeiro, sob o projeto $\mathrm{n}^{o}$ : 133153/2010-0.

Em especial, meus mais sinceros agradecimentos ao Luan que me amparou incondicionalmente, me deu impulso para seguir, me acalmou enquanto eu achava que tudo ia desmoronar, me animou quando perdi a coragem. Ao Luan, que foi a rocha mais sólida, a base mais firme.

Esta dissertação foi escrita em LATEX com a classe IAGTESE, para teses e dissertações do IAG. 
"Pouca gente, se é que alguém o fez, parece ter compreendido o mais autêntico princípio da realidade: novo conhecimento conduz sempre a mistérios ainda mais espantosos. Maior conhecimento fisiológico do cérebro torna a existência da alma menos possível, ainda que mais provável pela própria natureza da pesquisa."

Stephen King 



\section{Resumo}

Comparamos duas nuvens moleculares, Rosette (RMC) e Monoceros R2 (Mon R2), localizadas no Complexo de Monoceros, com o objetivo de estudar suas condições físicas relacionadas às primeiras fases da formação estelar. Tratam-se de regiões interessantes por apresentarem características que podem ser confrontadas com a hipótese de formação estelar provocada pela passagem de nuvens de altas velocidades atravessando o plano Galáctico (HVCs). Avaliamos as propriedades dessas nuvens por meio de mapas de vários traçadores da formação estelar com base em diferentes bandas espectrais visando estudar a estrutura de densidade das nuvens, bem como os objetos estelares jovens, em particular as fontes masers de $\mathrm{H}_{2} \mathrm{O}$ que apresentam características típicas de protoestrelas massivas. Nossa análise permitiu verificar algumas semelhanças entre RMC e Mon R2, mas também nos revelou diferenças interessantes. De uma forma geral há concordância entre $\mathrm{A}_{V}, \mathrm{CO}$ e emissão de poeira em $100 \mu \mathrm{m}$; RMC possui muitos clumps, entretanto poucos aglomerados e nebulosidades - exceto uma única região HII principal (NGC2244) - enquanto Mon R2 apresenta poucos clumps, vários aglomerados jovens e pequenas nebulosidades; em RMC há mais estrelas massivas, distribuídas uniformemente; Mon R2 tem poucas estrelas B, distribuídas em estruturas filamentárias com maiores índices de $\mathrm{A}_{V}$, do que em RMC; as fontes emissão masers apresentam cores IRAS compatíveis com candidatas a protoestrelas massivas, mas não parecem estar associadas a fontes de raios-X, sugerindo que masers estão relacionados à fase protoestelar, ao passo que fontes-X representam fase Pré-Sequência Principal. Concluímos que a distribuição de objetos e a estrutura das nuvens estão de acordo com as simulações dos modelos de HVCs. Porém, nossos resultados também são compatíveis com modelos alternativos, que simulam a dinâmica da Galáxia, para explicar o cenário de formação estelar no Complexo de Monoceros. 



\section{Abstract}

We compare two molecular clouds of the Monoceros Complex in order to study their physical conditions related to the early stages of star formation. The selected clouds, Rosette (RMC) and Monoceros R2 (Mon R2), are interesting regions due to their characteristics that may be confronted with the hypothesis of star formation triggered by high velocity clouds (HVCs) crossing through the Galactic plane. We evaluate the properties of these clouds using maps obtained on the basis of different spectral bands to trace the density of the clouds and the young stellar objects, in particular $\mathrm{H}_{2} \mathrm{O}$ masers that show typical features of massive protostars. This analysis allowed us to verify some similarities between RMC and Mon R2, but also revealed interesting differences. In a general way there is an agreement between $\mathrm{A}_{V}, \mathrm{CO}$ and dust emission at $100 \mu \mathrm{m}$; RMC has many clumps, a few clusters and a single main nebulosity that is an HII region around NGC2244, while Mon R2 has a few clumps, several young clusters and small nebulosities. In RMC there is a large number of massive stars, uniformly distributed, while Mon R2 has a few B stars, distributed in filamentary structures with levels of $\mathrm{A}_{V}$ higher than in RMC; maser sources have IRAS colors compatible with massive protostars candidates, but do not seem to be associated with X-ray sources, suggesting that masers are more related to the protostellar phase, while X-ray sources are related to pre main sequence phase. We conclude that the distribution of objects and the structure of the clouds are in accordance with the simulations of HVC models. However, our results are also compatible with alternative models of the Galaxy dynamics that explain the scenario of star formation in the Monoceros Complex. 



\section{Sumário}

1. Introdução . . . . . . . . . . . . . . . . . . . . . . . . . . . . 17

1.1 Características do meio interestelar . . . . . . . . . . . . . . . . . 18

1.2 As fases do meio interestelar . . . . . . . . . . . . . . . . . . . . . 18

1.3 Cenários de formação estelar: características gerais . . . . . . . . . . . . 20

1.4 Classificação dos objetos jovens: uma sequência evolutiva . . . . . . . . . . 21

1.5 Traçadores da formação estelar . . . . . . . . . . . . . . . . . . 22

1.6 A formação estelar no Complexo Orion-Monoceros . . . . . . . . . . . . . . 27

2. A Nuvem Molecular Rosette $(R M C)$. . . . . . . . . . . . . . . . . . . . . 33

2.1 Identificação óptica de estrelas massivas em RMC . . . . . . . . . . . . . . . 34

2.2 Detecção de estrelas jovens em Rosette por meio de raios-X . . . . . . . . . 38

2.3 Caracterização de aglomerados jovens, com base no infravermelho próximo 41

2.4 Distribuição de gás e poeira: mapas de CO e observações IRAS . . . . . 43

3. A Nuvem Molecular Monoceros (Mon R2) . . . . . . . . . . . . . . . . . . . . . 51

3.1 Identificação óptica de nebulosas de reflexão e aglomerados estelares . . . . 51

3.2 Detecção de estrelas jovens em Mon R2 por meio de raios-X . . . . . . . . 54

3.3 Caracterização de aglomerados jovens, com base no infravermelho próximo 57

3.4 Distribuição de gás molecular e associação com fontes IRAS . . . . . . . . 57

4. Metodologia e Análise Comparativa . . . . . . . . . . . . . . . . . . . 65

4.1 Distribuição das nuvens: mapas de extinção, gás molecular e poeira . . . 67

4.2 Comparação do conteúdo estelar com as regiões densas . . . . . . . . . . . 68 
4.3 Seleção das sub-regiões de interesse . . . . . . . . . . . . . . . . . . 71

4.4 Características das sub-regiões . . . . . . . . . . . . . . . . . . . . . 79

4.4.1 Análise no infravermelho próximo para RMC . . . . . . . . . . 79

4.4.2 Análise no infravermelho próximo para Mon R2 . . . . . . . . . 79

5. Discussão dos Resultados . . . . . . . . . . . . . . . . . . . . . . . . . . . . . . 85

5.1 Estrutura das nuvens . . . . . . . . . . . . . . . . . . . 88

5.2 Características dos clumps . . . . . . . . . . . . . . . . . . 91

5.3 Distribuição espacial da população estelar . . . . . . . . . . . . . . . . . 94

5.4 Estágio evolutivo das fontes $I R A S \ldots \ldots \ldots$

5.5 Aglomerados Jovens _ . . . . . . . . . . . . . . . . . . . . . . . . . . . . 97

5.6 O cenário de formação estelar no Complexo de Monoceros . . . . . . . . . 102

6. Considerações Finais . . . . . . . . . . . . . . . . . . . . . . 105

6.1 Conclusões . . . . . . . . . . . . . . . . . . . . . 105

6.2 Perspectivas . . . . . . . . . . . . . . . . . . . . 107

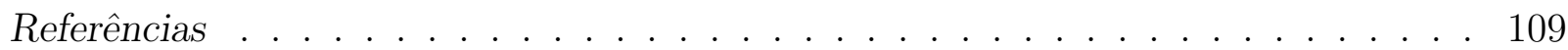

$\begin{array}{ll}\text { Apêndice } & 119\end{array}$

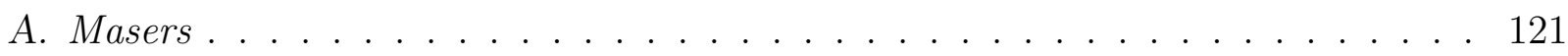

B. Busca de Fontes Maser no ROI . . . . . . . . . . . . . . . . . . . . . . . . . . . 125

B.1 O rádio telescópio . . . . . . . . . . . . . . . . . . . 125

B.1.1 Rastreio . . . . . . . . . . . . . . . . . 125

B.1.2 Aquisição . . . . . . . . . . . . . . . . . 126

B.1.3 Redução dos dados . . . . . . . . . . . . . . . . . 126

B.2 Observações . . . . . . . . . . . . . . . . . . . . . . . . . . . 128

B.2.1 Mon R2 . . . . . . . . . . . . . . . . . . . . . . . . . . . 128

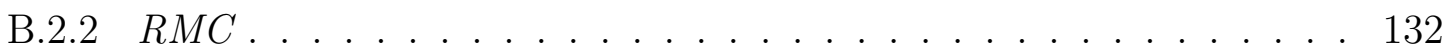




\section{Acrônimos}

- 2MASS: Two Micron All Sky Survey

- DSS: Digitized Sky Survey

- GMC: Giant Molecular Cloud

- GSC: Guide Star Catalog

- HVC: High Velocity Cloud

- IRAC: Infrared Array Camera

- IRAS: Infrared Astronomical Satellite

- ISIS: Imaging Speckle Interfereometer in Space

- MIPS: Multiband Imaging Photometer for Spitzer

- MS: Main Sequence

- MSX: Midcourse Space Experiment

- NTT: New Technology Telescope

- PMS: Pre Main Sequence

- POSS: Palomar Observatory Sky Survey

- PSC: Point Source Catalogue

- ROI: Rádio Observatório de Itapetinga

- ROSAT: Röngstensatellit

- RMC: Rosette Molecular Cloud

- SOFI: Son of ISAAC (espectrógrafo infravermelho e câmera de imageamento do NTT)

- UC HII: Ultra-Compact HII Region

- YSO: Young Stellar Object 
Capítulo 1

\section{Introdução}

O estudo das condições físicas que influenciam localmente as primeiras fases da formação estelar tem sido de grande interesse científico. Por um lado, os avanços tecnológicos e instrumentais proporcionaram um grande progresso na detecção de objetos protoestelares de baixa massa, possibilitando a realização de surveys e a construção de bancos de dados, principalmente no infravermelho. Por outro lado, os processos iniciais de formação de estrelas massivas são ainda pouco conhecidos e não há um cenário amplamente aceito (Zinnecker e Yorke, 2007).

Com o objetivo de apresentar o contexto em que se enquadra a pesquisa desenvolvida no presente trabalho, apresentamos neste capítulo uma introdução geral ao tema, dividindoo em três partes principais: (i) características típicas de regiões de formação de estrelas massivas e de baixa massa; (ii) classificação observacional dos objetos jovens, com base em um cenário evolutivo; (iii) diferentes traçadores da formação estelar. Neste caso, discutemse as diferentes bandas espectrais como base de dados para avaliar a estrutura de densidade das nuvens - mapas de extinção visual $\left(\mathrm{A}_{V}\right)$; emissão de poeira detectada no infravermelho distante e no milimétrico; distribuição do gás por meio de mapas de $\mathrm{CO}$ - bem como a busca e a identificação de objetos estelares jovens e aglomerados galácticos (associações OB, fontes infravermelhas, masers, fontes de raios-X). Finalmente, apresenta-se a motivação na escolha dos alvos estudados no presente trabalho, encontrados no complexo de nuvens moleculares em Monoceros. 


\subsection{Características do meio interestelar}

Todo o espaço que permeia as estrelas parece estar vazio. Na realidade, o que há nesse espaço é o chamado meio interestelar, constituido por diversos elementos. Esses elementos se combinam constituindo gás e poeira em grande quantidade. A partir dessa composição, os objetos interestelares são estruturados em regiões HII, nebulosas de reflexão, remanescentes de supernova e nuvens moleculares, por exemplo (Tielens, 2005). Todos esses objetos desempenham um papel crucial na evolução da Galáxia, pois a dinâmica desses componentes traduz química e fisicamente o enriquecimento do meio interestelar e, consequentemente, afeta de maneira direta a formação de novas gerações de estrelas a partir da morte de outras.

As regiões HII são, resumidamente, nebulosas de gás ionizado formadas pela influência de estrelas jovens massivas e quentes sobre o gás. As nebulosas de reflexão, por outro lado, refletem a luz de estrelas vizinhas. Os remanescentes de supernovas são formados quando uma estrela ejeta material por meio de uma explosão, no fim de sua vida.

Já as nuvens moleculares são percebidas como uma aparente falta de objetos na direção de determinadas regiões do céu, em comprimentos de onda ópticos. Na verdade, são densas nuvens formadas de gás molecular que absorvem a luz visível. Em condições específicas, essas grandes nuvens se tornam berçários de estrelas e por este motivo são os objetos abordados nesse trabalho.

\subsection{As fases do meio interestelar}

O meio interestelar é composto basicamente por poeira, moléculas e gás. A poeira interestelar é responsável pela absorção e espalhamento da luz das estrelas. Moléculas são constituídas nas regiões mais densas e, em muitos casos, as espécies se encontram e reagem sobre o grão de poeira para então serem ejetadas e voltar para o meio (Tielens, 2005). Já o gás se organiza no meio interestelar em uma variedade de fases.

As fases do gás no meio interestelar são caracterizadas pelas diferentes densidades e temperaturas. A Tabela 1.1 apresenta as propriedades físicas das fases do meio interestelar, de acordo com Kwok (2007).

Essencialmente, cada uma dessas fases pode ser caracterizada da seguinte forma: 
Tabela 1.1 - Componentes do meio interestelar e suas propriedades físicas. Tabela adaptada de Kwok (2007).

\begin{tabular}{|c|c|c|c|c|c|}
\hline $\begin{array}{l}\text { Componente do } \\
\text { meio interestelar }\end{array}$ & $\begin{array}{l}\text { Designações } \\
\text { comuns }\end{array}$ & $\begin{array}{l}\text { Temperatura } \\
(\mathrm{K})\end{array}$ & $\begin{array}{c}\text { Densidade }\left[\mathrm{n}_{H_{2}}\right] \\
\left(\mathrm{cm}^{-3}\right)\end{array}$ & $\begin{array}{l}\text { Estado do } \\
\text { hidrogênio }\end{array}$ & $\begin{array}{l}\text { Diagnósticos } \\
\text { típicos }\end{array}$ \\
\hline Meio ionizado quente & Gás coronal & $10^{6}$ & 0.003 & $\mathrm{H}^{+}$ & Emissão de raios-X, Absorção UV. \\
\hline Meio ionizado morno & Gás ionizado difuso & $10^{4}$ & $>10$ & $\mathrm{H}^{+}$ & Óptico, UV, IV, H $\alpha$, regiões HII. \\
\hline Meio neutro morno & HI internuvens & $8 \times 10^{3}-10^{4}$ & 0.1 & $\mathrm{H}^{0}$ & Emissão em $21 \mathrm{~cm}$ \\
\hline Meio neutro atômico morno & Nuvens difusas & 100 & $10-100$ & $\mathrm{H}^{0}+\mathrm{H}_{2}$ & $21 \mathrm{~cm}$ em absorção, $3.4 \mu \mathrm{m}$ em absorção. \\
\hline Meio neutro frio molecular & Nuvens moleculares & 1 & $10^{3}-10^{5}$ & $\mathrm{H}_{2}$ & $\begin{array}{l}\text { Extinção, IV distante, } \\
\text { rádio }\left(\mathrm{CO}, \mathrm{CS}, \mathrm{NH}_{3} \ldots\right)\end{array}$ \\
\hline Cores moleculares quentes & Cores protoestelares & $100-300$ & $>10^{6}$ & $\mathrm{H}_{2}$ & $\begin{array}{c}\text { Emissão rotovibracional } \\
\left(\mathrm{CH}_{3} \mathrm{CN}, \mathrm{CH}_{3} \mathrm{OH}, \mathrm{HCN} \ldots\right) .\end{array}$ \\
\hline
\end{tabular}

- Gás Coronal: aquecido e ionizado por choques impulsionados por ventos estelares e explosões de supernovas;

- Gás Ionizado: possui baixa densidade e sofre incidência direta de radiação ultravioleta e raios cósmicos;

- Gás Neutro: localizado em ambientes quentes intra-nuvens ou organizado em nuvens HI frias difusas na forma atômica;

- Gás Molecular: disposto em nuvens escuras e nuvens moleculares gigantes, formando sítios de formação estelar ativa.

A Figura 1.1 representa um esquema ilustrativo das fases do meio interestelar.

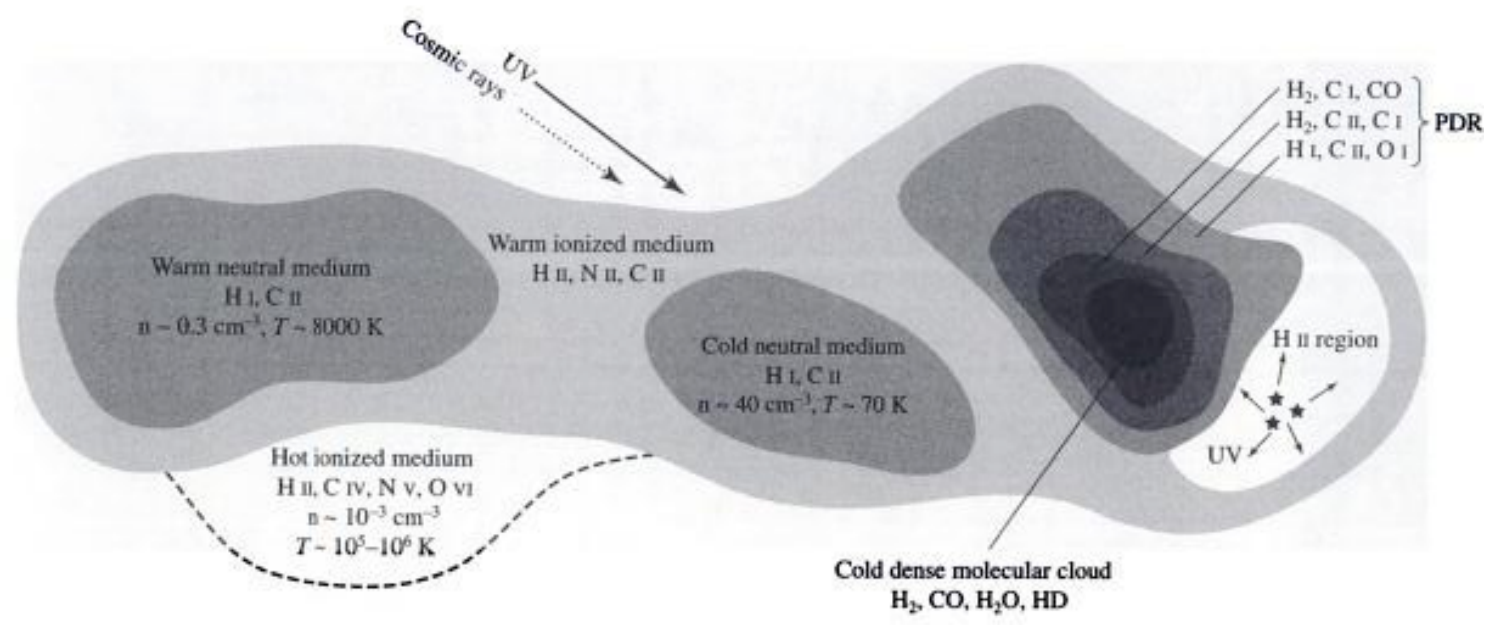

Figura 1.1: Diagrama esquemático das fases do meio interestelar, extraído de Kwok (2007). 
O estudo e a caracterização das propriedades das nuvens moleculares permitem conhecer melhor os estágios iniciais dos processos de formação estelar, uma vez que estrelas se formam a partir do colapso gravitacional de tais nuvens.

\subsection{Cenários de formação estelar: características gerais}

Enquanto alguns trabalhos teóricos propõem um modelo de formação de estrelas massivas $\left(\mathrm{M}_{*}>8 \mathrm{M}_{\odot}\right)$ como sendo uma versão similar aos processos de colapso de nuvem e subsequente acresção, que ocorre na formação de estrelas de baixa massa (McKee e Tan, 2003), outros processos como acresção competitiva e coalescência de protoestrelas de massa intermediária $\left(\mathrm{M}_{*} \sim 2-8 \mathrm{M}_{\odot}\right)$ são igualmente propostos (Bonnell et al., 2004). Uma sequência empírica tem sido identificada, desde condensações de nuvens escuras observadas no infravermelho, passando para núcleos densos e mais quentes de nuvens, formando regiões desde hipercompactas, passando para compactas e finalmente chegando às clássicas regiões HII. Entretanto, ainda não está claro qual dos modelos teóricos é mais adequado para este cenário.

Por outro lado, os estágios iniciais dos processos de formação de estrelas de baixa massa $\left(\mathrm{M}_{*}<2 \mathrm{M}_{\odot}\right)$ podem ser melhor compreendidos por meio da caracterização e do estudo das propriedades de pequenas condensações que são formadas a partir do colapso gravitacional de nuvens moleculares. Os trabalhos que utilizam observações milimétricas de nuvens galácticas classificam essas condensações (de acordo com o termo original) como:

- starless core: gás e poeira concentrada, sem a presença de estrelas,

- prestellar core: ainda sem estrelas, mas com densidades maiores, e

- protostar: protestrelas, conhecidas como objetos de Classe 0.

Bergin e van Dishoeck (2012) discutem a presença de água nas diferentes fases de formação de estrelas e planetas, que assume a forma de vapor, em condições turbulentas e de alta temperatura associadas a estrelas massivas, ou a forma de mantos de gelo em grãos, presentes nos discos protoplanetários. Nesse contexto, Bergin e van Dishoeck (2012) apresentam o quadro geral dos estágios de formação estelar e das condições físicas dessas regiões, resumindo e exemplificando muito bem as condições físicas das regiões e 
objetos relacionados ao nosso trabalho. A Tabela 1.2 resume tais características e a Figura 1.2 mostra nossa proposta esquemática, para apresentar como se interligam as diferentes fases, desde grandes complexos de nuvens moleculares gigantes (GMCs) até formar a região HII, no caso de estrelas massivas (OB). Para estrelas de baixa massa, do tipo T Tauri, ou de massa intermediária (Herbig $\mathrm{Ae} / \mathrm{Be}$ ), que neste trabalho nos referimos de forma genérica como estrelas pré-sequência principal $(P M S)$, as etapas são melhor conhecidas e classificadas de acordo com a evolução da estrutura circunstelar que leva à formação de um disco protoplanetário.

Tabela 1.2 - Propriedades físicas relevantes do gás associado a diferentes estágios de formação estelar. Tabela adaptada de Bergin e van Dishoeck (2012).

\begin{tabular}{|c|c|c|c|c|c|c|}
\hline & \multirow[b]{2}{*}{$\begin{array}{c}\text { Nuvem } \\
\text { Molecular }\end{array}$} & \multirow[b]{2}{*}{$\begin{array}{c}\text { Clump } \\
\text { (prestellar core) }\end{array}$} & \multicolumn{3}{|c|}{ Protoestrela } & \multirow[b]{2}{*}{ Disco } \\
\hline & & & Core quente & Fluxo extenso & Gás sob choque & \\
\hline$\rho\left(\mathrm{cm}^{-3}\right)$ & $\sim 10^{3}$ & $10^{5}-10^{6}$ & $>10^{6}$ & $10^{3}-10^{4}$ & $10^{5}-10^{6}$ & $10^{6}-10^{15}$ \\
\hline $\mathrm{T}(\mathrm{K})$ & $10-20$ & $\sim 10$ & $100-300$ & $10-100$ & $100-1000$ & $10-2000$ \\
\hline $\mathrm{M}\left(\mathrm{M}_{\odot}\right)$ & $10^{3}$ a $10^{5}$ & $10-10^{3} c$ & $10^{-2 d}$ & $\ldots$ & $\ldots$ & $10^{-3}-1^{e}$ \\
\hline $\mathrm{v}\left(\mathrm{km} \mathrm{s}^{-1}\right)^{a}$ & $1-3$ & $0.3-1.5$ & $5-15$ & $5-15$ & $10-50$ & $1-5$ \\
\hline $\mathrm{D}^{b}$ & $>10 \mathrm{pc}$ & $0.1 \mathrm{pc}$ & $20 \mathrm{R}_{\odot}$ & $0.1-1 \mathrm{pc}$ & $\ldots$ & 100 - 1000 U.A. \\
\hline
\end{tabular}

Rádio-telescópios modernos, operando em comprimentos de onda sub-milimétricos, oferecem altas sensibilidades e resolução espacial necessárias para detectar condensações frias e compactas, que têm propriedades intermediárias entre os fragmentos de nuvens (clumps) mais difusos, detectados em CO, e os YSOs infravermelhos (André et al., 2000; Ward-Thompson et al., 2007). Esse tipo de detalhamento observacional permitiu estabelecer uma sequência evolutiva, com fases separadas de acordo com as mudanças no regime de opacidade da matéria circunstelar, como descreveremos a seguir.

\subsection{Classificação dos objetos jovens: uma sequência evolutiva}

A evolução na $P M S$ ocorre em estágios marcados pela diminuição da massa do envoltório circunstelar à medida que a massa do $Y S O$ aumenta por acresção a partir do prestellar core André et al. (2007). Assim, objetos Classe 0 possuem $M_{*}<<M_{e n v}$, apresentando um máximo de emissão nos comprimentos de onda milimétricos (André et al., 1993) e evoluem para YSOs Classe I com $M_{*}<M_{e n v}$ (Lada, 1987; André e Montmerle, 1994) 


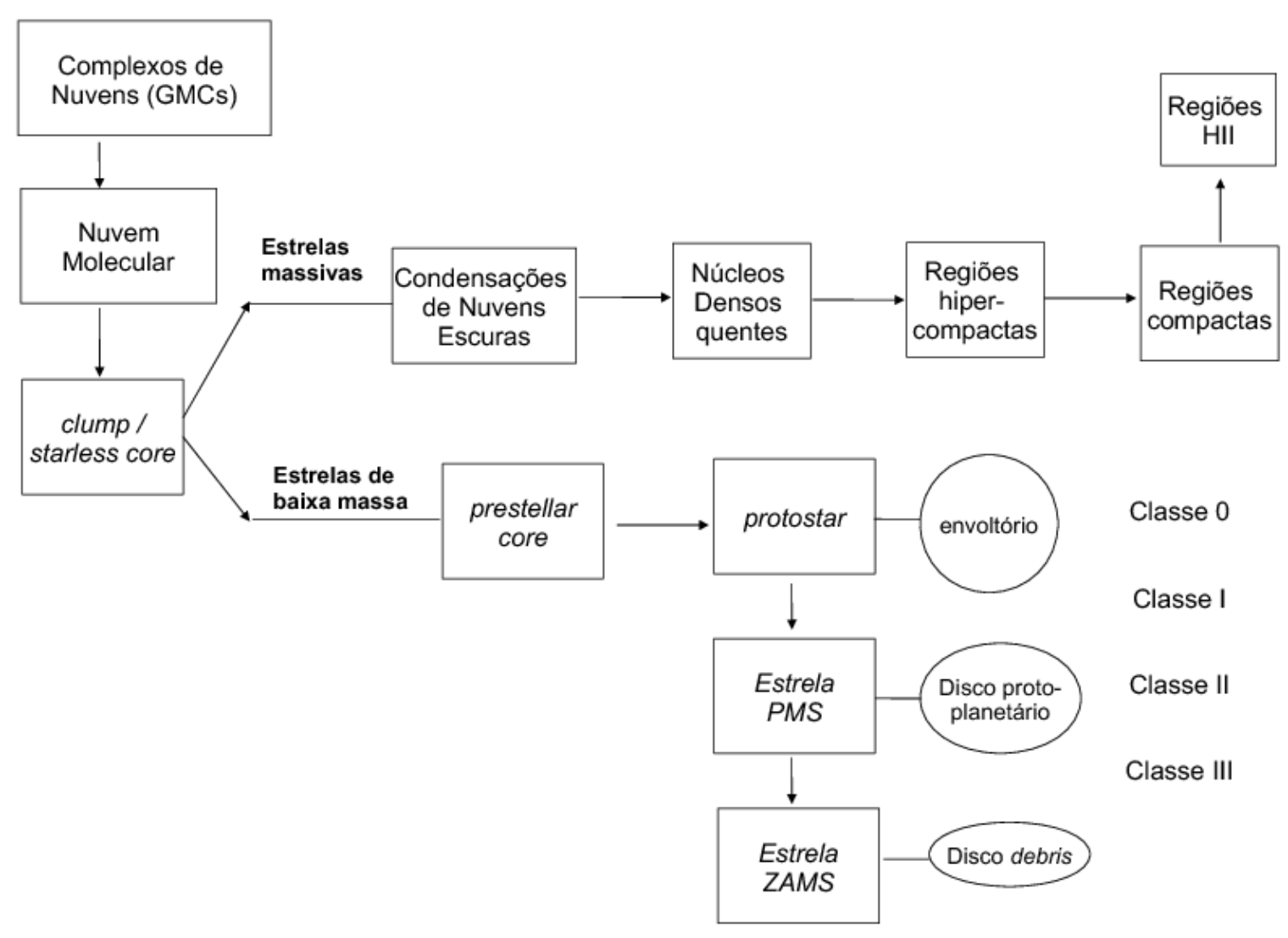

Figura 1.2: Esquema ilustrativo das diferentes etapas de formação estelar. Os detalhes das etapas de formação de estrelas massivas são menos conhecidos do que no caso das estrelas de baixa massa. Para estas, uma sequência evolutiva é proposta com base na quantidade de material circunstelar, de Classe 0 (mais embebida) até Classe III (desaparecimento do disco/formação de planetas).

emitindo principalmente no infravermelho. Efeitos combinados da rotação e da queda de material em acresção promovem a ejeção de material na direção dos polos e a sedimentação do material circunstelar no plano equatorial, formando um disco ao redor de uma estrela visível, que passa a ser considerada uma estrela PMS Classe II ou III $\left(M_{e n v} \sim 0\right)$. O esquema da Figura 1.3, adaptado de Feigelson e Montmerle (1999), ilustra as diferentes características das Classes 0 a III.

\subsection{Traçadores da formação estelar}

Uma das formas de buscar por estrelas jovens é através da detecção de raios-X, graças aos intensos processos energéticos típicos da juventude estelar. A emissão de raios-X é 


\begin{tabular}{|c|c|c|c|c|c|}
\hline Properties & $\begin{array}{l}\text { Infalling } \\
\text { Protostar }\end{array}$ & $\begin{array}{l}\text { Evolved } \\
\text { Protostar }\end{array}$ & $\begin{array}{c}\text { Classical } \\
\text { T Tauri } \\
\text { Star }\end{array}$ & $\begin{array}{c}\text { Weak-lined } \\
\text { T Tauri } \\
\text { Star }\end{array}$ & $\begin{array}{c}\text { Main } \\
\text { Sequence } \\
\text { Star }\end{array}$ \\
\hline SKetCh & $\frac{x^{\prime}}{4}$ & $\frac{1}{\therefore /}$ & $\frac{1}{11}$ & $\frac{i}{i+i}$ & $\circ 0$ \\
\hline $\begin{array}{c}\text { AGE } \\
\text { (YEARS) }\end{array}$ & $10^{4}$ & $10^{5}$ & $10^{6}-10^{7}$ & $10^{6}-10^{7}$ & $>10^{7}$ \\
\hline $\begin{array}{c}\text { mm/lNFRARED } \\
\text { CLASS }\end{array}$ & Class 0 & Class I & Class II & Class III & (Class III) \\
\hline Disk & Yes & Thick & Thick & $\begin{array}{c}\text { Thin or } \\
\text { Non-existent }\end{array}$ & $\begin{array}{c}\text { Possible } \\
\text { Planetary } \\
\text { System }\end{array}$ \\
\hline X-RAY & $?$ & Yes & Strong & Strong & Weak \\
\hline $\begin{array}{c}\text { THERMAL } \\
\text { RADIO }\end{array}$ & Yes & Yes & Yes & No & No \\
\hline $\begin{array}{c}\text { NON-THERMAL } \\
\text { RADIO }\end{array}$ & No & Yes & No? & Yes & Yes \\
\hline
\end{tabular}

Figura 1.3: Características das etapas na pré-sequência principal e as categorias estabelecidas com observações milimétricas e no infravermelho. Figura extraída de Feigelson e Montmerle (1999).

associada com atividade magnética e/ou ventos estelares (Kohno et al., 2002) e é proporcional à luminosidade bolométrica (Berghöefer et al., 1997). Isso pode ser atribuído ao aquecimento do plasma pelo choque induzido através do vento estelar (Lucy e White, 1980).

Objetos estelares jovens de baixa massa apresentam espectro e variabilidade temporal de emissão em raios-X similares aos observados para as estrelas do tipo solar, mas com uma luminosidade muito maior (Feigelson e Decampli, 1981; Montmerle et al., 1983). Conforme a estrela evolui para a $M S$, a intensidade da emissão de raios-X diminui.

No caso das estrelas T Tauri, a luminosidade em raios-X $\left(\mathrm{L}_{X}\right)$ com relação a luminosidade bolométrica $\left(\mathrm{L}_{b o l}\right)$ é $\mathrm{L}_{X} / \mathrm{L}_{b o l} \sim 10^{-4}$, enquanto que para massas maiores, como nas Herbig Ae/Be, é de $\mathrm{L}_{X} / \mathrm{L}_{b o l} \sim 10^{-6}$. Assim, a busca por grandes amostras de estrelas jovens de baixa massa é muito mais eficiente via detecção de raios-X do que no visível. 
As buscas baseadas na emissão $\mathrm{H} \alpha$, por exemplo, foram realizadas com sucesso na direção de regiões de formação estelar próximas, como Taurus, Chamaeleon e $\rho$ Ophiucus, entre outras, localizadas a cerca de 150 pc. No entanto, em regiões mais distantes, a baixa luminosidade das T Tauri faz com que elas fiquem abaixo do limite de detecção dos surveys ópticos. Com o advento dos satélites de raios-X, foi possível identificar um número consideravelmente maior de estrelas T Tauri, como indicam, por exemplo, resultados alcançados com o ROSAT All Sky Survey (Alcalá et al., 1995).

Mais recentemente, o poder das observações de raios-X em revelar grandes amostras de estrelas jovens tornou-se bem estabelecido para nuvens de distância intermediária, até alguns kpc (Gregorio-Hetem et al., 2009), e pode ser ilustrado pelos trabalhos realizados com o satélite Chandra, como por exemplo o projeto COUP ("Chandra Orion Ultradeep Project") (Getman et al., 2005), que revelou mais de 1600 fontes de raios-X associadas ao aglomerado estelar da Nebulosa de Orion. Outro exemplo é o trabalho de Wang et al. (2008), um estudo da região M17, que se encontra a cerca de $1.5 \mathrm{kpc}$, onde foram detectadas pelo Chandra cerca de 1000 fontes-X.

Entre as regiões estudadas com o satélite XMM-Newton destacam-se por exemplo Vela OB2 (Jeffries et al., 2009); Orion (López-Santiago e Caballero, 2008); e o projeto XEST ("XMM-Newton Extended Survey of Taurus") (Guedel et al., 2007). Em cada nuvem, milhares de fontes pontuais tem sido detectadas, quase todas identificadas com estrelas $P M S$ com massas até o limite de anãs marrons.

No domínio do infravermelho distante, tem sido muito amplo o uso dos dados em comprimentos de onda mais longos, para mapear a emissão fria de poeira interestelar ou circunstelar, bem como para detectar a emissão morna de poeira nas proximidades da estrela rodeada por um envoltório e/ou disco circunstelar. Neste caso, em particular, tem sido de grande importância a disponibilidade dos dados do Catálogo 2MASS (Two Micron All Sky Survey) (Skrutskie et al., 2006) na análise do estágio evolutivo das estrelas PMS, bem como de aglomerados estelares jovens.

Da mesma forma, temos o levantamento realizado pelo IRAS (Infrared Astronomical Satellite) nos comprimentos de onda na faixa do infravermelho distante (12, 25, 60 e 100 $\mu \mathrm{m})$ que permitiu a sensibilidade adequada para a detecção de objetos de baixa temperatura e pouca luminosidade, fornecendo um meio satisfatório de se buscar candidatas a 
protoestrelas (Beichman et al., 1986). Imagens obtidas pelo IRAS possibilitam verificar a presença de objetos embebidos em regiões de formação estelar.

Posteriormente, outros surveys forneceram dados com melhor sensibilidade e maior faixa espectral, embora com menor abrangência espacial, tais como o MSX ("Midcourse Space Experiment") (Price et al., 1994), ISO, Akari. Spitzer e Herschel, fornecendo dados fotométricos e/ou espectroscópicos que têm permitido análises detalhadas tanto dos objetos estelares quanto do meio que os circundam.

Motte et al. (2010) apresentam um levantamento de YSOs de massa intermediária e alta massa, denominado HOBYS ("Herschel imaging survey of OB Young Stellar objects"). Utilizando observações nas bandas entre 70 e $500 \mu \mathrm{m}$, foram identificados 46 prestellar cores de alta massa. A Figura 1.4 mostra os cores mais massivos da nuvem molecular Rosette, classificando a natureza dos mesmos e estimando seu estágio evolutivo.

A emissão rádio devida a transições moleculares também é uma excelente traçadora da formação estelar, tanto pelos mapeamentos de densidade, temperatura e velocidades das nuvens moleculares, via detecção de $\mathrm{CO}, \mathrm{NH}_{3}$, CS, etc., quanto pela detecção da emissão maser, comumente associada às estrelas jovens massivas.

No estudo de evidências que traçam a formação de estrelas, a identificação de emissão maser se mostra importante por geralmente ser observada na direção de envoltórios de objetos estelares jovens nas fases mais iniciais de sua formação. Através da intensidade da emissão maser é possível obter informações a respeito das condições físicas do meio que abriga tais objetos (Reid e Moran, 1981). A emissão maser tem sido, com efeito, observada no envoltório molecular de estrelas em formação, em seus estágios mais primordiais (Breen et al., 2010) e o fato de estarem altamente embebidas não impede que sejam detectadas na faixa de radiofrequências. Um maior detalhamento dos processos físicos relacionados com a emissão maser é apresentado no Apêndice A.

Finalmente, destaca-se a importância das observações polarimétricas, que revelam a distribuição e o alinhamento de grãos que permeiam tanto o meio interestelar quanto o material circunstelar. Rodrigues et al. (2009), por exemplo, realizaram um estudo dos dados polarimétricos de uma centena de estrelas Herbig Ae/Be. Embora o trabalho tenha detectado polarização intrínseca em boa parte da amostra estudada, revelando uma simetria não esférica na estrutura circunstelar de tais objetos, os resultados mais interessantes 


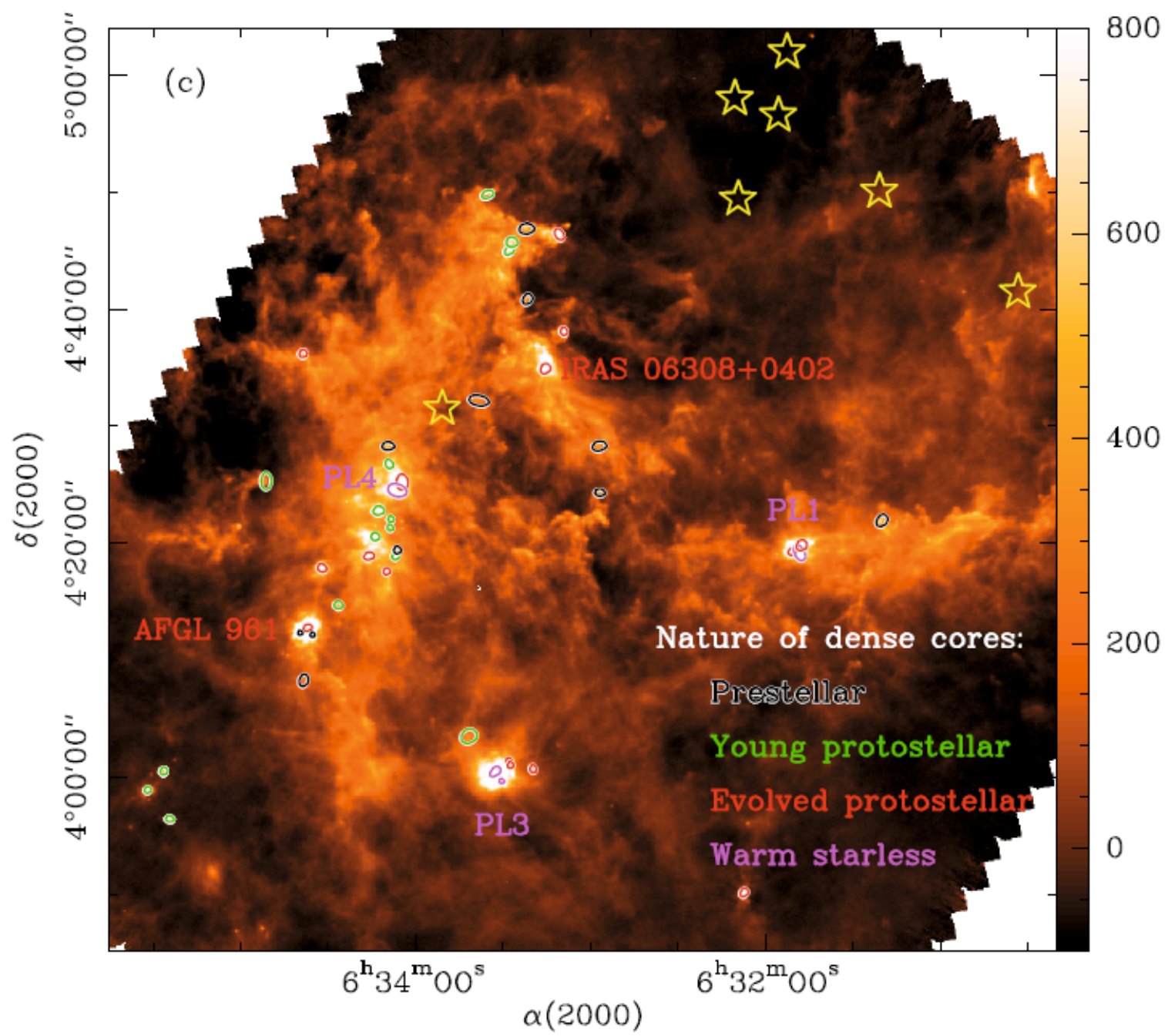

Figura 1.4: Distribuição espacial dos cores massivos presentes em Rosette sobre um mapa de $160 \mu \mathrm{m}$ detectados pelo satélite Herschel. O aglomerado OB NGC2244 está marcado com estrelas, enquanto as cinco fontes $\operatorname{IRAS}$ com $\mathrm{L}_{I R}>10^{3} \mathrm{~L}_{\odot}$ aparecem indicadas em vermelho e pink. Figura extraída de Motte et al. (2010)

foram focados no meio ambiente interestelar. Para os objetos mais polarizados, os autores verificaram uma correlação entre o eixo de simetria do material circunstelar das Herbig Ae/Be e a direção do campo magnético interestelar, um indicativo do efeito do meio ambiente no achatamento da estrutura circunstelar e na definição de seu eixo de momento angular.

Um outro exemplo da correlação do campo magnético com a morfologia de nuvens escuras, evidenciada pelo mapeamento polarimétrico, foi apresentado por Pereyra e Magalhães (2004) para Musca, uma nuvem filamentária encontrada na região de Chamaeleon. 
Mais recentemente, os estudos polarimétricos tem sido dedicados ao detalhamento dos diferentes tipos de grãos presentes em discos circunstelares, servindo-se de dados obtidos no infravermelho, como no caso do trabalho de Pereyra et al. (2012), dedicado ao estudo da estrutura circunstelar do sistema binário PDS144, selecionado entre as Herbig Ae/Be previamente estudadas por Pereyra et al. (2009).

\subsection{A formação estelar no Complexo Orion-Monoceros}

Como mencionado acima, os surveys em raios-X e a exploração dos dados IRAS propiciaram uma revolução no estudo de grandes amostras de estrelas jovens, que ocorreu no final da década de 80. Naquela mesma época, os mapeamentos de CO propiciaram diversos estudos da distribuição de gás na Galáxia e, consequentemente, a estrutura em grande escala dos principais centros de formação estelar.

Uma proposta bastante interessante para explicar a presença de regiões de formação estelar fora do plano Galáctico foi baseada na hipótese de nuvens moleculares a altas velocidades $(H V C s)$, provenientes de altas latitudes que colidem contra o plano Galáctico e o atravessam (Mirabel e Franco, 1981).

O choque contra o gás e o arrasto desse material explicariam a presença de estrelas jovens, bem como a estrutura filamentária das nuvens localizadas a altas latitudes Galácticas. Franco et al. (1988), por exemplo, apresentaram um modelo, explicado pelas HVCs, para a origem comum dos complexos moleculares de Orion e Monoceros, que se encontram a cerca de 100 pc distantes do plano Galáctico.

Lépine e Duvert (1994) propõem um modelo semelhante para explicar a estrutura filamentária e inclinada de nuvens como Chamaeleon, Ophiucus, Orion e Taurus-AurigaPerseus, formada pela passagem de HVCs. Enquanto tais trabalhos realizaram simulações bidimensionais, Hetem (1995) apresenta resultados mais detalhados, obtidos com simulações 3D, que confirmaram as previsões de formação de GMCs a 200 pc abaixo do disco Galáctico, cerca de 10 milhões de anos após o choque de $H C V s$, que também explicam a distribuição espacial de estrelas $\mathrm{OB}$ nessas regiões.

Para ilustrar alguns casos de nuvens que se encontram abaixo do disco Galáctico, apresentamos na Figura 1.5 um mapa de extinção visual $\left(\mathrm{A}_{V}\right)$, extraído do catálogo de Dobashi et al. (2005), cobrindo longitudes galácticas de $\sim 190^{\circ}$ a $320^{\circ}$. Nota-se neste 
quadrante galáctico os complexos de nuvens em baixas latitudes, como em Chamaeleon: Cha I, II, III $\left(\mathrm{b} \sim-15^{\circ}\right)$ e Musca $\left(\mathrm{b} \sim-10^{\circ}\right)$, e o Complexo Orion-Monoceros $\left(\mathrm{b}<-12^{\circ}\right)$. Como pode ser verificado na Figura 1.5, a distribuição das regiões mais densas em Chamaeleon e também em Orion-Monoceros tendem a ser filamentárias e inclinadas, de acordo com os modelos de HVCs mencionados acima. Essas estruturas também são correlacionadas com as observações de CO, de acordo com o mapa apresentado no trabalho de Bloemen et al. (1990) e reproduzidas no painel superior da Figura 1.6. No painel inferior desta mesma figura apresentamos o resultado do modelo utilizado por Hetem $(1995,1996)$, que reproduz a distribuição de densidades no complexo Orion-Monoceros, adotando-se a hipótese da passagem de uma $H V C$.

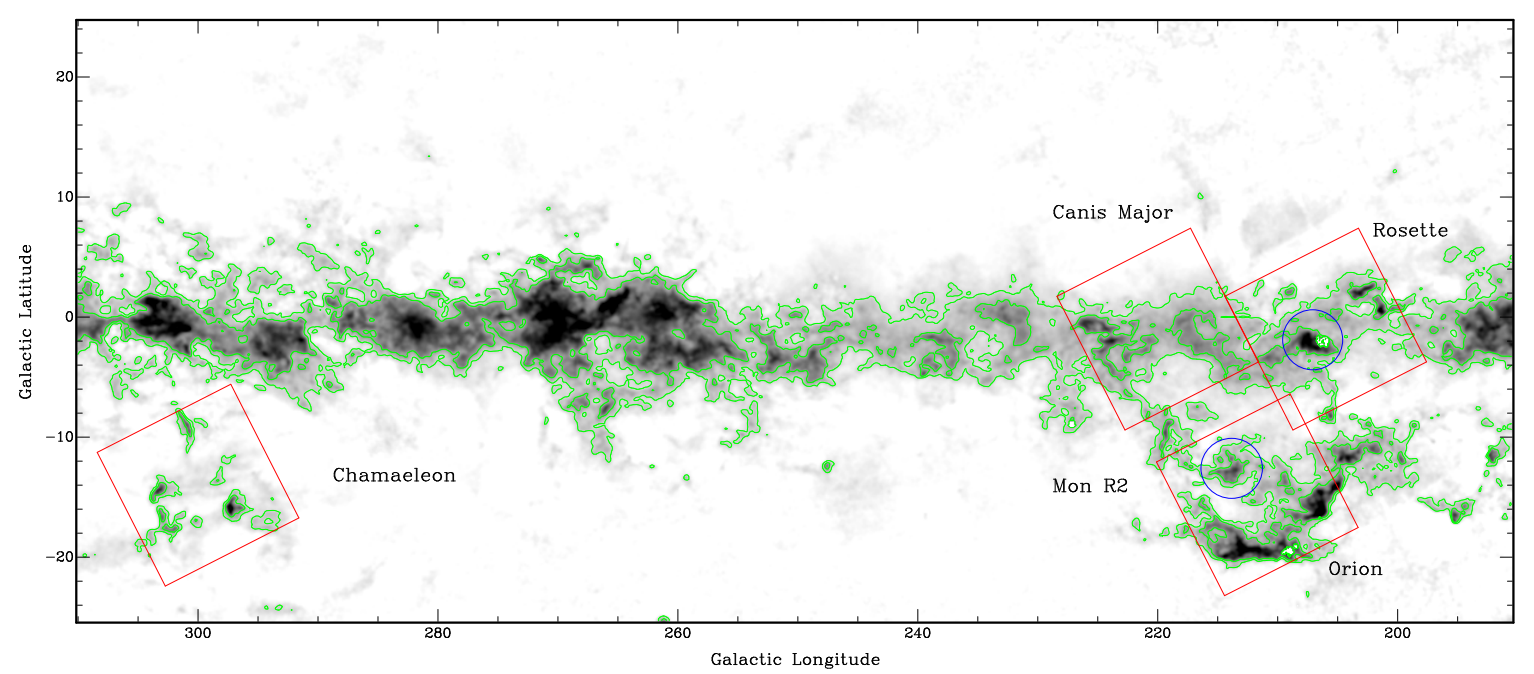

Figura 1.5: Mapa de extinção visual indicando os contornos de $\mathrm{A}_{V}=0.5$ e 1.0 mag. Destaque para alguns complexos de nuvens moleculares (losângulos vermelhos) e para as nuvens Rosette e Mon R2 (círculos azuis, com raio de $5^{\circ}$ ), estudadas neste trabalho (vide Capítulos 2 e 3 ).

Também destacam-se na Figura 1.5 as GMCs Canis Major, Mon R2 e Rosette, que foram estudadas por Gregorio-Hetem et al. $(1998,2010)$ com base na detecção de fontes de raios-X pelo satélite ROSAT. A maioria destas fontes foi identificada como estrelas $P M S$, propiciando um melhor detalhamento da distribuição de massas nestas GMCs, que previamente eram conhecidas como formadoras unicamente de estrelas de alta massa. 

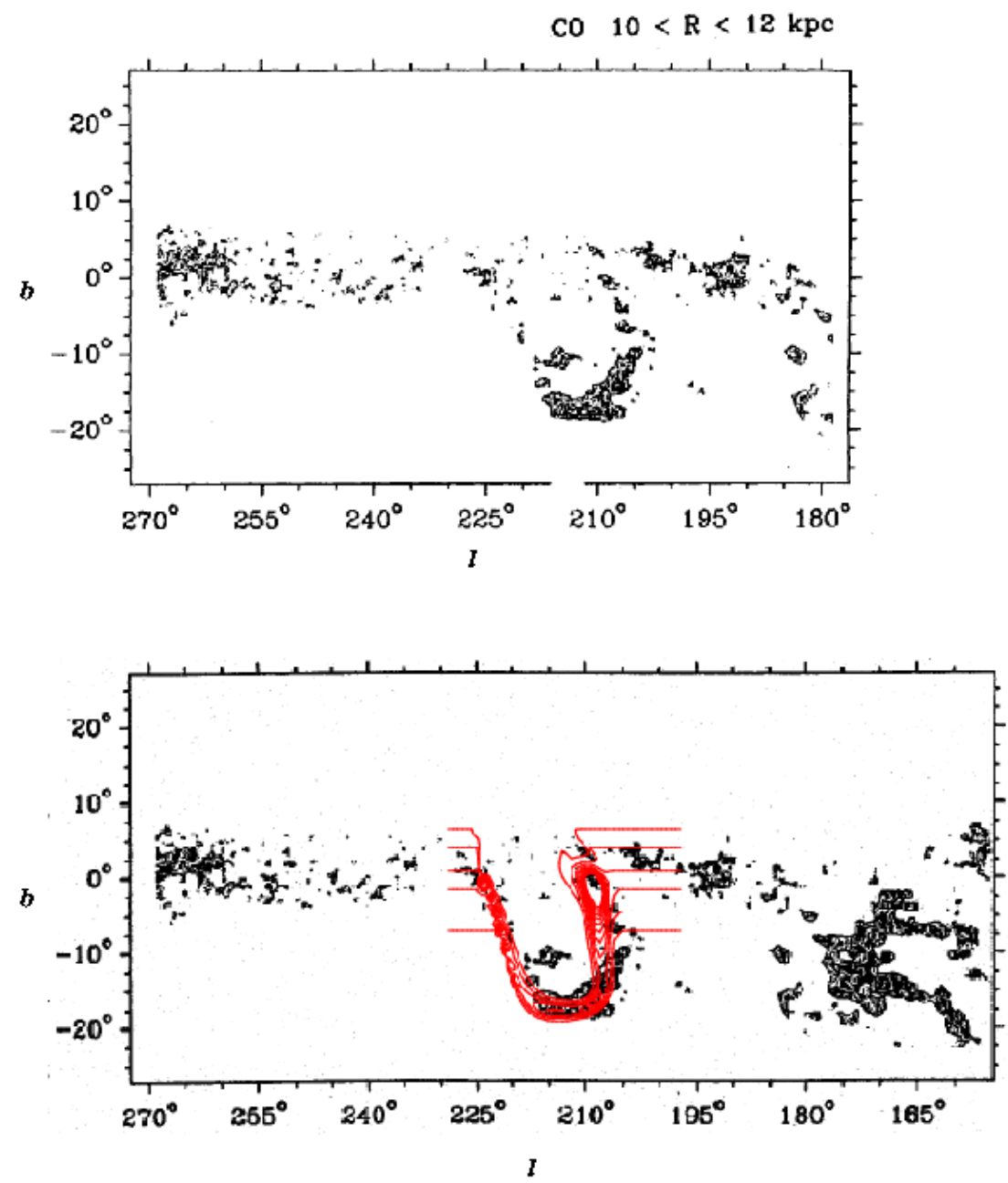

Figura 1.6: O painel da superior apresenta parte do mapa de CO, extraído do trabalho de Bloemen et al. (1990), mostrando a distribuição de gás no complexo de nuvens moleculares na região Orion-Monoceros. No painel inferior são apresentados os resultados do modelo de $H V C s$ que sinteticamente reproduz a distribuição de CO na mesma região, de acordo com o calculado por Hetem (1996).

Estrelas OB mostram forte influência nos efeitos da formação de estrela de baixa massa, como revelam alguns estudos sobre a origem de estrelas massivas (Parker e Goodwin, 2007; Preibisch e Zinnecker, 1999; Dolan e Mathieu, 2002). A determinação da Função de Massa Inicial nesses estudos se mostra importante na comparação entre diversas regiões de formação estelar.

Motivados pelas características interessantes acima descritas para as GMCs localizadas 
no Complexo de Monoceros, realizamos no presente trabalho um estudo comparativo que busca relacionar as condições físicas das nuvens com a distribuição das estrelas jovens de diferentes massas. O levantamento de objetos associados a nuvens moleculares, cujas propriedades são consistentes com regiões de formação estelar, nos permite explorar sua natureza e conhecer melhor o cenário de evolução de protoestrelas presentes nessas regiões, para que estudos mais detalhados possam ser realizados de forma estatística. Assim, é possível obter um conhecimento mais aprofundado das condições de formação e evolução de protoestrelas.

Ao se comparar as GMCs entre si, procura-se revelar diferenças e semelhanças que possam contribuir para um melhor entendimento do cenário de formação estelar no qual se inserem essas nuvens. Apesar de se tratarem de regiões já muito estudadas, em comprimentos de onda desde raios-X até rádio, ainda não foi realizado um levantamento sistematizado de suas características, visando um estudo comparativo e em grande escala das condições de formação estelar no contexto do Complexo de Monoceros, mais especificamente na região da associação Mon OB2 (vide Capítulo 4). A combinação de dados rádio, milimétricos e infravermelhos torna possível a verificação de evidências do estágio evolutivo dos YSOs associados a nuvens, em particular os grandes complexos moleculares que podem apresentar vários proto-aglomerados.

Para apresentar a distribuição espacial dos objetos notáveis encontrados na região de nosso interesse, apresentamos na Figura 1.7 uma composição de imagens ópticas, extraídas do DSS, mostrando algumas estrelas brilhantes das constelações de Orion e Canis Major, bem como algumas nebulosas de emissão. Para mostrar a distribuição das nuvens associadas a estas regiões, elaboramos um mosaico baseado nas imagens compostas IRAS-ISIS (bandas 12, 25, 60 e $100 \mu \mathrm{m}$ ), que revelam a poeira morna, devida a presença de estrelas, bem como a poeira fria, devida a presença de nuvens escuras, como pode ser visto na Figura 1.8. Ambas figuras ilustram de forma geral e introdutória o que se pretende discutir em detalhe no presente trabalho. 


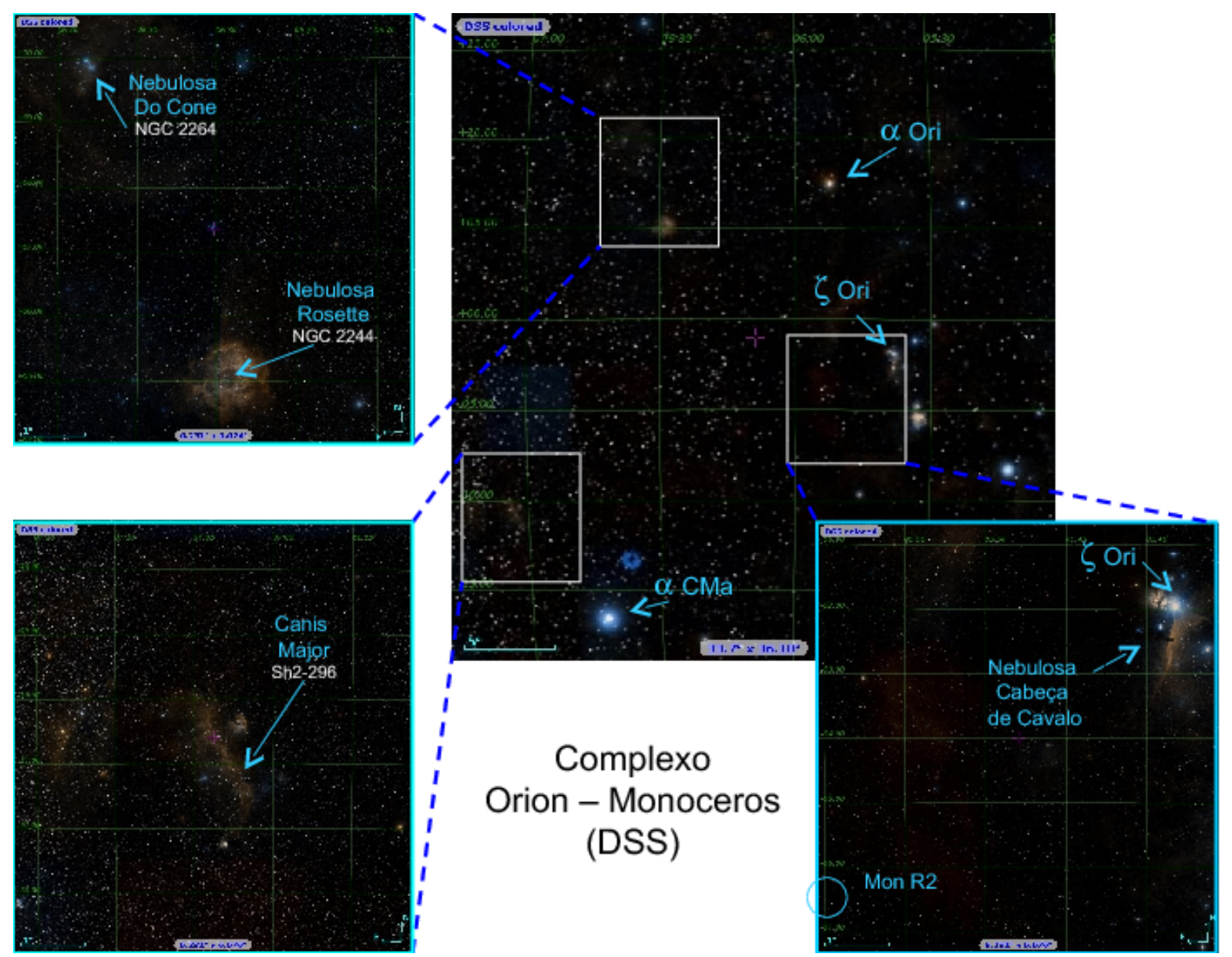

Figura 1.7: Visão geral da região onde se localiza o complexo Orion-Monoceros de nuvens moleculares, com base na imagem óptica obtida pelo DSS. Para referência, indica-se a posição das estrelas Betelgeuse ( $\alpha$ Ori), Anitak ( $\zeta$ Ori) e Sirius ( $\alpha$ CMa); e das nebulosas e/ou aglomerados estelares (Rosette/NGC2244, Nebulosa do Cone/NGC2264, Mon R2 e Canis Major/Sh2-296). Mapas extraídos do atlas ALADIN (http://aladin.u-strasbg.fr/aladin.gml).

A estrutura da dissertação divide-se nas seguintes partes. Os Capítulos 2 e 3 são dedicados à descrição das características das nuvens Rosette e Mon R2 (respectivamente) e os grupos estelares, escolhidos como principais alvos de estudo neste trabalho. No Capítulo 4 analisamos e comparamos todas as informações coletadas para essas nuvens, em diversos domínios espectrais. Tais resultados são discutidos no Capítulo 5, enquanto que no Capítulo 6 resumimos as conclusões alcançadas e discutimos as perspectivas da continuidade do trabalho. No Apêndice A apresentamos um resumo das características físicas da emissão maser, dada a importância de sua associação às nossas regiões de interesse. No Apêndice B descrevemos o andamento de um projeto de radio-observações de masers, relacionado ao presente trabalho, que está sendo desenvolvido em paralelo. 


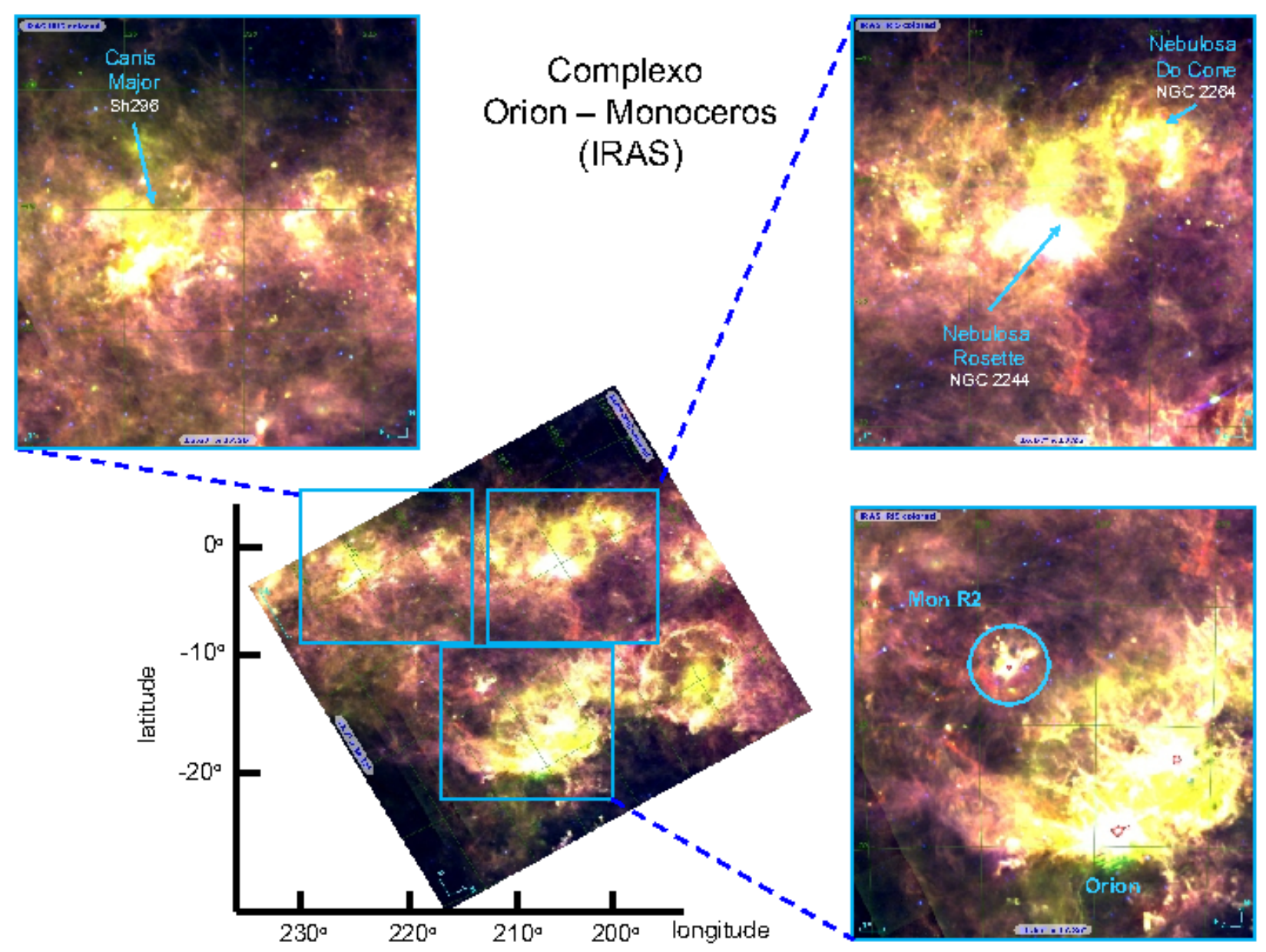

Figura 1.8: Idem à Figura 1.7, com base na imagem composta a partir dos dados IRAS, na qual as cores mais claras indicam emissão morna, devida a radiação estelar, e as cores escuras mostram a emissão fria de poeira. O painel central ilustra o quadro geral do complexo Orion-Monoceros, no sistema de coordenadas equatoriais (B1950) comparado às coordenadas galácticas, visando destacar as principais regiões (quadros laterais em zoom).

Finalmente, destaca-se que neste trabalho utilizamos o ponto decimal para padronização das informações provindas de diversos trabalhos e necessidade de introduzir tais dados em programas de computador e catálogos de busca nesse formato. Salientamos, ainda, que os termos em inglês foram adotados devido a sua ampla utilização na área e a tradução direta nem sempre se mostra suficiente. Uma lista de acrônimos usualmente adotados apresenta-se na página 15. 
Capítulo 2

\section{A Nuvem Molecular Rosette (RMC)}

Como mencionado no capítulo anterior, a motivação na escolha das nuvens moleculares no Complexo de Monoceros foi inspirada em sua localização privilegiada na Galáxia, dada a hipótese do choque de $H V C s$ provocando uma distribuição peculiar das nuvens e das estrelas recém formadas, distantes do disco galáctico. Além disso, o trabalho baseado nas observações ROSAT mostrou que a população jovem de baixa massa era conhecida de forma incompleta, indicando a presença de estrelas do tipo T Tauri nessas GMCs onde apenas estrelas massivas haviam sido identificadas anteriormente (Gregorio-Hetem et al., 1998). Assim, o estudo comparativo das nuvens Rosette - que se encontra no plano da Galáxia - e Mon R2, que está a cerca de $-12^{\circ}$ do disco galáctico, nos revela diferenças e semelhanças que podem contribuir para a melhor compreensão do cenário de formação estelar no Complexo de Monoceros.

A literatura estabelece a base necessária para a compreensão da região. Recentemente, Bo Reipurth organizou uma compilação intitulada Handbook of Star Forming Regions, que reúne (em dois volumes) informações das mais importantes regiões de formação estelar. Os livros contêm uma excelente caracterização das regiões selecionadas e dos aglomerados jovens associados, mostrando a riqueza de detalhes a respeito das condições físicas das nuvens moleculares estudadas. Entre os temas revisados nessa obra, um é artigo dedicado à RMC, a nuvem molecular gigante Rosette (Román-Zúñiga e Lada, 2008). Um outro artigo é dedicado à nuvem molecular gigante Mon R2 (Carpenter e Hodapp, 2008). Por meio desses trabalhos, é possível realizar um levantamento sistemático das características da região, visando um estudo comparativo e em grande escala das condições de formação estelar no contexto do Complexo de Monoceros. 
Neste capítulo e nos próximos apresenta-se a coletânea de dados realizada para cada nuvem, separando-se as informações por domínio espectral. Também como identificamos e selecionamos alguns dos objetos interessantes (protoestrelas, estrelas massivas, aglomerados estelares) associados a essas regiões, que foram incluídos na análise comparativa realizada no presente trabalho.

\subsection{Identificação óptica de estrelas massivas em RMC}

Resultados prévios obtidos para RMC mostram que se trata de uma região muito interessante para se estudar a formação estelar, dada sua complexidade e sua variedade de condições físicas, tanto propícias à formação de aglomerados estelares jovens de baixa massa, como estrelas massivas e de massa intermediária. A Figura 2.1 apresenta a localização de Rosette no contexto do Complexo de Monoceros. 


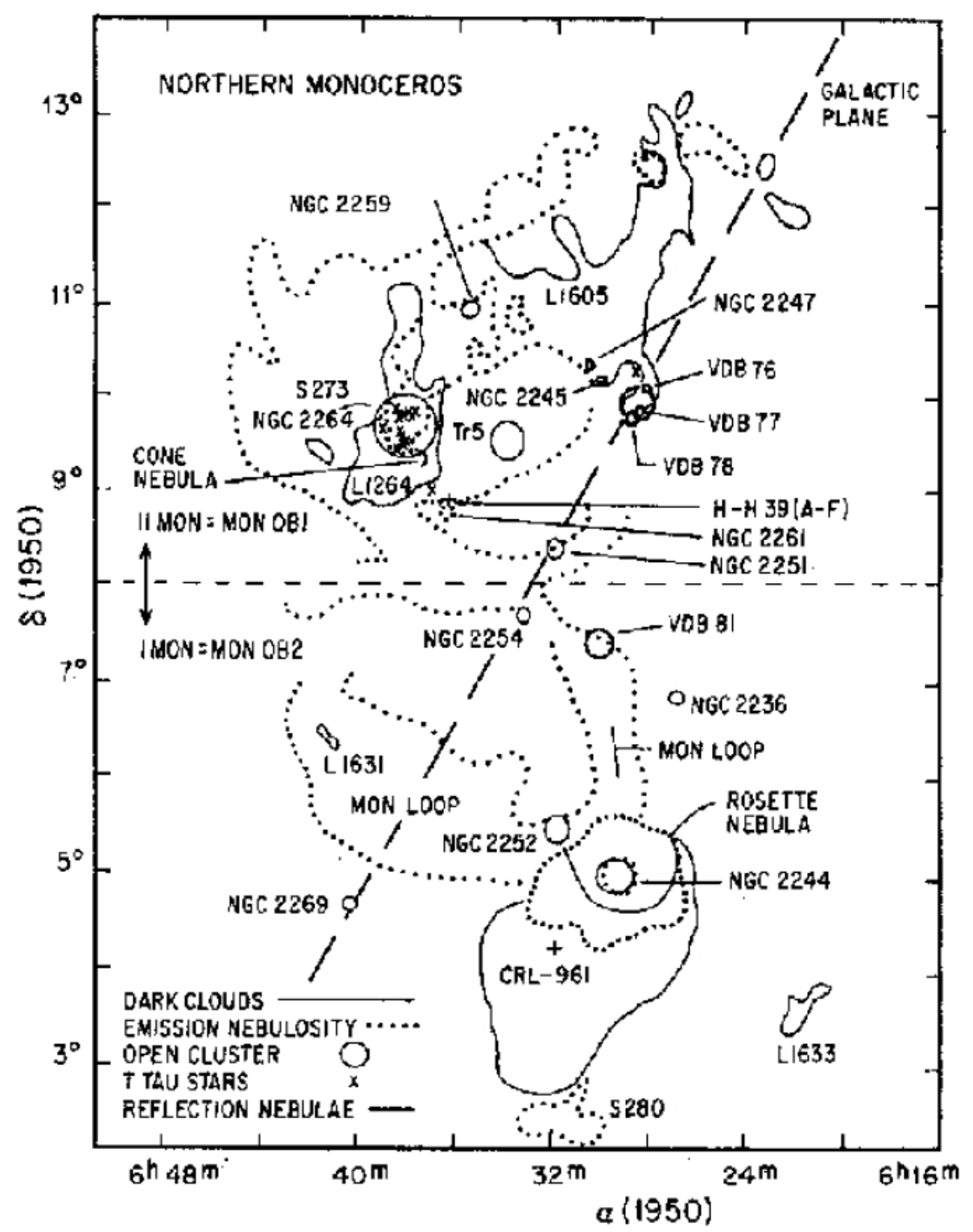

Figura 2.1: Localização de Rosette, indicando a posição de nuvens escuras e da Nebulosa Rosette. Figura de Pérez (1991).

A Tabela 2.1 traz a população de estrelas OB (7 estrelas tipo O, e 24 tipo B) contida no aglomerado NGC2244, no centro da Nebulosa Rosette, enquanto a Tabela 2.2 lista outros algomerados jovens localizados nas suas proximidades, os quais foram listados no trabalho de Román-Zúñiga e Lada (2008) com base em uma coletânea realizada a partir de trabalhos anteriores. Na Seção 2.4 discutiremos a distribuição de clumps detectados em RMC por Williams et al. (1995). A Tabela 2.2 também apresenta o raio dos clumps aos quais se associam alguns dos aglomerados jovens. 
Tabela 2.1 - Membros OB do aglomerado NGC2244, localizado na nuvem molecular Rosette. Tabela adaptada de Román-Zúñiga e Lada (2008).

\begin{tabular}{|c|c|c|c|c|c|c|}
\hline Identifição Óptica & $\begin{array}{c}1 \\
\left({ }^{\circ}\right)\end{array}$ & $\begin{array}{l}\mathrm{b} \\
\left({ }^{\circ}\right)\end{array}$ & $\begin{array}{c}\text { A.R. }(\mathrm{J} 2000) \\
(\mathrm{h} \mathrm{m} \mathrm{s})\end{array}$ & $\begin{array}{c}\operatorname{Dec}(\mathrm{J} 2000) \\
\left({ }^{\circ}, "\right)\end{array}$ & Tipo Espectral & $\begin{array}{c}\mathrm{m}_{V} \\
(\mathrm{mag})\end{array}$ \\
\hline HD 46223 & 206.2617 & -02.0425 & 06:32:09.32 & $+04: 49: 24.6$ & $\mathrm{O} 4 \mathrm{~V}$ & 7.32 \\
\hline HD 46150 & 206.1821 & -02.0410 & $06: 31: 55.52$ & $+04: 56: 34.3$ & $\mathrm{O} 5 \mathrm{~V}$ & 6.75 \\
\hline HD 46485 & 206.5351 & -01.5011 & $06: 33: 50.95$ & $+04: 31: 31.6$ & $\mathrm{O} 7 \mathrm{~V}$ & 8.20 \\
\hline HD 46056 & 206.2009 & -01.1450 & $06: 31: 20.87$ & $+04: 50: 03.9$ & $\mathrm{O} 8 \mathrm{~V}$ & 8.16 \\
\hline HD 46149 & 206.1312 & -02.0220 & $06: 31: 52.54$ & $+05: 01: 59.1$ & $08.5 \mathrm{~V}$ & 7.59 \\
\hline HD 258691 & 206.2219 & -02.2919 & $06: 30: 33.31$ & $+04: 41: 27.6$ & O9V & 9.70 \\
\hline HD 46202 & 206.1848 & -02.0012 & $06: 32: 10.48$ & $+04: 57: 59.7$ & O9V & 8.20 \\
\hline HD 259238 & 206.1456 & -01.5601 & $06: 32: 18.22$ & $+05: 03: 21.7$ & Bov & 11.10 \\
\hline HD 46106 & 206.1155 & -02.0538 & $06: 31: 38.40$ & $+05: 01: 36.3$ & $\mathrm{~B} 0.2 \mathrm{~V}$ & 7.95 \\
\hline MJD95 & 206.4285 & -02.2195 & 06:31:37.08 & $+04: 45: 53.7$ & $\mathrm{~B} 0.5 \mathrm{~V}$ & 15.15 \\
\hline HD 259135 & 206.2223 & -02.0450 & $06: 32: 00.61$ & $+04: 52: 41.0$ & B $0.5 \mathrm{~V}$ & 8.54 \\
\hline GSC 00154-00234 & 206.3659 & -01.4514 & $06: 33: 37.49$ & $+04: 48: 47.0$ & $\mathrm{~B} 0.5 \mathrm{~V}$ & 11.89 \\
\hline HD 259012 & 206.2104 & -02.1146 & $06: 31: 33.46$ & $+04: 50: 39.7$ & $\mathrm{~B} 1 \mathrm{~V}$ & 9.35 \\
\hline HD 259105 & 206.1830 & -02.0514 & $06: 31: 52.00$ & $+04: 55: 57.3$ & $\mathrm{~B} 1 \mathrm{~V}$ & 9.42 \\
\hline GSC $00154-02337$ & 206.2324 & -02.0349 & $06: 32: 06.13$ & $+04: 52: 15.3$ & B1III & 9.73 \\
\hline HD 46484 & 206.4657 & -01.4538 & $06: 33: 54.41$ & $+04: 39: 44.6$ & $\mathrm{~B} 1 \mathrm{~V}$ & 7.65 \\
\hline $\mathrm{BD}+051281 \mathrm{~B}$ & 206.1933 & -02.0350 & $06: 31: 58.93$ & $+04: 55: 39.9$ & $\mathrm{~B} 1.5 \mathrm{~V}$ & 10.38 \\
\hline HD 259172 & 206.1133 & -01.5839 & $06: 32: 02.59$ & $+05: 05: 08.6$ & $\mathrm{~B} 2 \mathrm{~V}$ & 10.71 \\
\hline GSC $00154-02247$ & 206.1805 & -01.4406 & $06: 33: 06.56$ & $+05: 06: 03.4$ & B2 & 12.85 \\
\hline GSC 00154-02504 & 206.2031 & -02.1204 & $06: 31: 31.47$ & $+04: 50: 59.6$ & $\mathrm{~B} 2.5 \mathrm{~V}$ & 10.64 \\
\hline GSC 00154-02141 & 206.1929 & -02.0654 & $06: 31: 47.89$ & $+04: 54: 18.1$ & $\mathrm{~B} 2.5 \mathrm{~V}$ & 11.66 \\
\hline GSC $00154-02187$ & 206.1900 & -02.0334 & $06: 31: 58.91$ & $+04: 56: 16.2$ & $\mathrm{~B} 2.5 \mathrm{~V}$ & 11.26 \\
\hline GSC 00154-01007 & 206.1459 & -01.5824 & $06: 32: 09.84$ & $+05: 02: 13.4$ & $\mathrm{~B} 2.5 \mathrm{~V}$ & 11.22 \\
\hline GSC 00154-01016 & 206.3006 & -02.0212 & $06: 32: 24.24$ & $+04: 47: 03.7$ & $\mathrm{~B} 2.5 \mathrm{~V}$ & 11.41 \\
\hline GSC 00154-01247 & 206.2704 & -01.3625 & $06: 33: 50.56$ & $+05: 01: 37.6$ & $\mathrm{~B} 2.5 \mathrm{~V}$ & 11.12 \\
\hline GSC 00154-01753 & 206.2144 & -02.0020 & $06: 32: 15.49$ & $+04: 55: 20.4$ & B3 & 12.01 \\
\hline MJD95 & 206.3723 & -01.9776 & $06: 32: 22.49$ & $+04: 55: 34.2$ & B3V & 15.39 \\
\hline HD 259268 & 206.1301 & -01.5514 & $06: 32: 23.04$ & $+05: 02: 45.7$ & B3 & 11.09 \\
\hline HD 259300 & 206.2155 & -01.5631 & $06: 32: 29.39$ & $+04: 56: 56.1$ & B3V & 10.79 \\
\hline MJD95 & 206.4004 & -01.7695 & $06: 33: 10.16$ & $+04: 59: 49.9$ & B3V & 14.98 \\
\hline GSC 00154-02164 & 206.3304 & -01.5601 & $06: 32: 51.79$ & $+04: 47: 16.2$ & B5V & 12.88 \\
\hline
\end{tabular}


Tabela 2.2 - Aglomerados jovens localizados na nuvem molecular Rosette. Tabela adaptada de Román-Zúñiga e Lada (2008).

\begin{tabular}{|c|c|c|c|c|c|}
\hline Identificação ${ }^{(1)}$ & $\begin{array}{c}1 \\
\left({ }^{\circ}\right)\end{array}$ & $\begin{array}{c}\mathrm{b} \\
\left({ }^{\circ}\right)\end{array}$ & $\begin{array}{l}\text { A.R. (J2000) } \\
\quad(\mathrm{h} \mathrm{m} \mathrm{s})\end{array}$ & $\begin{array}{c}\operatorname{Dec}(\mathrm{J} 2000) \\
\left({ }^{\circ}, "\right)\end{array}$ & $\begin{array}{l}\mathrm{R}_{\text {clump }} \\
(\mathrm{pc})\end{array}$ \\
\hline PL01 & 206.5027 & -02.2249 & $06: 31: 49.32$ & $+04: 19: 34.7$ & 2.21 \\
\hline PL02 & 206.4616 & -01.5546 & $06: 33: 16.78$ & $+04: 35: 32.1$ & 1.33 \\
\hline PL03 & 207.197 & -02.086 & 06:33:33.07 & $+04: 00: 11.2$ & 2.79 \\
\hline PL04 & 207.0154 & -01.4827 & $06: 34: 13.92$ & $+04: 25: 05.4$ & 2.36 \\
\hline PL05 & 207.0843 & -01.4700 & $06: 34: 30.70$ & $+04: 20: 01.8$ & 1.50 \\
\hline PL06 & 207.1556 & -01.4825 & $06: 34: 38.76$ & $+04: 12: 55.4$ & 3.06 \\
\hline PL07 & 207.3410 & -01.4305 & $06: 35: 29.78$ & $+03: 59: 10.9$ & 2.79 \\
\hline REFL08 & 207.0722 & -01.4927 & $06: 34: 18.98$ & $+04: 20: 03.6$ & 1.71 \\
\hline REFL09 & 207.47 & -01.56 & $06: 35: 07.73$ & $+03: 41: 34.7$ & 3.60 \\
\hline PouC & 206.4955 & -01.5853 & $06: 33: 12.68$ & $+04: 31: 00.5$ & - \\
\hline PouD & 207.1713 & -02.0505 & $06: 33: 40.98$ & $+04: 03: 56.1$ & - \\
\hline REFL10 & 205.55 & -02.06 & 06:31:06.79 & $+05: 14: 50.0$ & - \\
\hline NGC2237 & 206.2032 & -02.0444 & $06: 31: 58.51$ & $+04: 54: 35.7$ & - \\
\hline NGC2244 & 206.0712 & -02.2040 & $06: 30: 36.10$ & $+04: 58: 50.6$ & - \\
\hline \multicolumn{6}{|c|}{$\begin{array}{l}\text { Notas: (1) Identificações correspondem às referências: PL - Phelps e Lada (1997) } \\
\text { REFL - Román-Zúñiga (2006); Pou - Poulton et al. (2008). }\end{array}$} \\
\hline
\end{tabular}




\subsection{Detecção de estrelas jovens em Rosette por meio de raios-X}

A nebulosa Rosette, uma famosa região HII escavada por fortes ventos de estrelas dos tipos $\mathrm{O}$ e B, é parte de um complexo maior - uma nuvem molecular que se estende por $\sim 100$ pc. Gregorio-Hetem et al. (1998) centraram o campo do satélite ROSAT no sudoeste da região HII, onde há densas concentrações de gás molecular, originalmente vistas em mapas de CO construídos por Blitz e Thaddeus (1980). Foram detectadas 21 fontes pontuais de raios-X, 14 delas com contrapartidas no GSC (Guide Star Catalog) do Telescópio Espacial Hubble, listadas na Tabela 2.3. A Figura 2.2 mostra contornos de raios-X, resultantes das observações realizadas pelo ROSAT, sobrepostos em imagem óptica POSS (R) cobrindo uma área de $2^{\circ}$ x $2^{\circ}$.

Posteriormente, Townsley et al. (2003) conduziram um estudo da emissão em raios$\mathrm{X}$ em regiões HII galácticas com base nos dados do Satélite Chandra, portanto com uma sensibilidade maior que aquela das observações $R O S A T$, mas com abrangência espacial bem menor (campos de $17^{\prime}$ x 17'). Na região central da Nebulosa Rosette eles encontraram mais de 300 fontes pontuais. Além disso, foi detectada emissão difusa de gás quente (0.7 a 8 M Kelvin), proveniente de ventos de estrelas $\mathrm{O}$ em choque com o meio interestelar. No segundo artigo da série, Wang et al. (2009) apresentaram uma análise detalhada dos dados Chandra para a Nebulosa Rosette e identificaram, com base nos dados infravermelhos, os aglomerados jovens associados às fontes de raios-X, permitindo inferir a presença de 1700 estrelas jovens na região.

Para ilustrar a distribuição espacial das fontes detetadas pelo Chandra, em comparação com as observações ROSAT, o painel a direita da Figura 2.2 apresenta os resultados obtidos por Wang et al. (2009).

Tabela 2.3 - Fontes pontuais ROSAT (raios-X) associadas a RMC. Tabela baseada em Gregorio-Hetem et al. (1998).

\begin{tabular}{|c|c|c|c|c|c|c|c|c|}
\hline $\begin{array}{c}\text { No. } \\
-\end{array}$ & $\begin{array}{c}\text { Nome } \\
\text { RXJ }\end{array}$ & $\begin{array}{c}1 \\
\left({ }^{\circ}\right)\end{array}$ & $\begin{array}{c}\mathrm{b} \\
\left({ }^{\circ}\right)\end{array}$ & $\begin{array}{c}\mathrm{AR} \\
(\mathrm{h} \mathrm{m} \mathrm{s})\end{array}$ & $\begin{array}{c}\text { Dec } \\
\left({ }^{\circ}, "\right)\end{array}$ & $\begin{array}{c}\text { Cnts/Ks } \\
- \\
\end{array}$ & $\begin{array}{l}\log \mathrm{L}_{X} \\
(\mathrm{erg} / \mathrm{s})\end{array}$ & $\begin{array}{c}\text { Identificação óptica } \\
-\end{array}$ \\
\hline 1 & $0631.5+0403$ & 207.05 & -02.57 & $6: 31: 31.3$ & 4:03:31 & $2.7 \pm 1.1$ & $30.82 \pm 0.20$ & GSC 0154-01486 \\
\hline 2 & $0632.1+0447$ & 206.46 & -02.09 & $6: 32: 06.5$ & $4: 47: 50$ & $19.1 \pm 2.2$ & $31.67 \pm 0.07$ & $\ldots$ \\
\hline 3 & $0632.3+0451$ & 206.43 & -02.02 & $6: 32: 19.5$ & $4: 51: 35$ & $8.5 \pm 1.5$ & $31.31 \pm 0.10$ & GSC 0154-02464 \\
\hline 4 & $0632.6+0349$ & 207.40 & -02.42 & $6: 32: 41.7$ & $3: 49: 12$ & $15.9 \pm 1.9$ & $31.59 \pm 0.07$ & GSC $0154-02220^{(1)}$ \\
\hline 5 & $0632.8+0444$ & 206.59 & -01.95 & $6: 32: 53.3$ & $4: 44: 24$ & $7.6 \pm 1.4$ & $31.27 \pm 0.10$ & $\ldots$ \\
\hline 6 & $0633.2+0446$ & 206.61 & -01.88 & $6: 33: 12.1$ & $4: 46: 29$ & $22.7 \pm 2.2$ & $31.74 \pm 0.07$ & GSC $0154-00114^{(2)}$ \\
\hline 7 & $0633.3+0410$ & 207.15 & -02.10 & $6: 33: 22.0$ & $4: 10: 34$ & $3.1 \pm 0.9$ & $30.88 \pm 0.15$ & $\ldots$ \\
\hline 8 & $0633.8+0431$ & 206.89 & -01.84 & $6: 33: 50.5$ & $4: 31: 34$ & $10.6 \pm 1.5$ & $31.41 \pm 0.09$ & GSC $0154-01414^{(3)}$ \\
\hline
\end{tabular}


Tabela 2.3 - continuação.

\begin{tabular}{ccccccccc}
\hline \hline No. & Nome & $\begin{array}{c}\mathrm{l} \\
\text { RXJ }\end{array}$ & $\begin{array}{c}\mathrm{b} \\
\left.{ }^{\circ}\right)\end{array}$ & $\begin{array}{c}\mathrm{AR} \\
\left({ }^{\circ}\right)\end{array}$ & $\begin{array}{c}\text { Dec } \\
(\mathrm{h} \mathrm{m} \mathrm{s})\end{array}$ & $\begin{array}{c}\text { Cnts/Ks } \\
\left({ }^{\circ},\right)\end{array}$ & $\begin{array}{c}\log L_{X} \\
(\mathrm{erg} / \mathrm{s})\end{array}$ & $\begin{array}{c}\text { Identificação óptica } \\
-\end{array}$ \\
\hline 9 & $0634.0+0419$ & 207.09 & -01.88 & $6: 34: 03.3$ & $4: 19: 40$ & $2.0 \pm 0.6$ & $30.69 \pm 0.16$ & GSC 0154-02156 \\
10 & $0634.1+0429$ & 206.96 & -01.79 & $6: 34: 07.3$ & $4: 29: 19$ & $1.5 \pm 0.6$ & $30.56 \pm 0.20$ & GSC 0154-00020 \\
11 & $0634.2+0428$ & 206.99 & -01.77 & $6: 34: 16.5$ & $4: 28: 01$ & $10.2 \pm 1.2$ & $31.39 \pm 0.08$ & $\ldots$ \\
12 & $0634.3+0403$ & 207.37 & -01.94 & $6: 34: 21.5$ & $4: 03: 35$ & $4.5 \pm 0.8$ & $31.04 \pm 0.10$ & GSC 0154-00622 \\
13 & $0634.5+0419$ & 207.16 & -01.77 & $6: 34: 33.4$ & $4: 19: 15$ & $5.0 \pm 0.9$ & $31.08 \pm 0.10$ & $\ldots$ \\
14 & $0634.6+0422$ & 207.13 & -01.73 & $6: 34: 38.3$ & $4: 22: 04$ & $2.4 \pm 0.7$ & $30.76 \pm 0.14$ & $(5)$ \\
15 & $0634.8+0400$ & 207.46 & -01.85 & $6: 34: 49.8$ & $4: 00: 45$ & $1.9 \pm 0.6$ & $30.66 \pm 0.16$ & GSC 0154-01958 \\
16 & $0634.9+0405$ & 207.39 & -01.79 & $6: 34: 54.5$ & $4: 05: 53$ & $1.5 \pm 0.5$ & $30.56 \pm 0.17$ & GSC 0154-02560 \\
17 & $0635.3+0428$ & 207.12 & -01.55 & $6: 35: 19.8$ & $4: 28: 05$ & $3.3 \pm 0.9$ & $30.90 \pm 0.14$ & $\ldots$ \\
18 & $0635.7+0424$ & 207.21 & -01.47 & $6: 35: 45.3$ & $4: 24: 48$ & $2.8 \pm 1,1$ & $30.83 \pm 0.20$ & $\ldots$ \\
19 & $0636.0+0418$ & 207.34 & -01.44 & $6: 36: 06.1$ & $4: 18: 53$ & $5.7 \pm 1.3$ & $31.14 \pm 0.12$ & GSC 0154-01628 \\
20 & $0636.1+0404$ & 207.56 & -01.53 & $6: 36: 10.3$ & $4: 04: 51$ & $5.5 \pm 1.1$ & $31.12 \pm 0.11$ & $\ldots$ \\
21 & $0636.9+0408$ & 207.59 & -01.33 & $6: 36: 54.7$ & $4: 08: 54$ & $9.3 \pm 1.5$ & $31.35 \pm 0.09$ & GSC 0154-00732 \\
\hline
\end{tabular}

Outras identificações: (1) HD $46301\left(\mathrm{~F} 5, m_{V}=7.8\right)$; (2) HD 259512 (F6, $m_{V}=10$ ) e IRAS 06306+0448; (3) HD $46485\left(\mathrm{O} 8, m_{V}=8.58\right)$ e IRAS 06312+0434; (4) IRAS 0614+0421; (5) IRAS 06321+0424. 


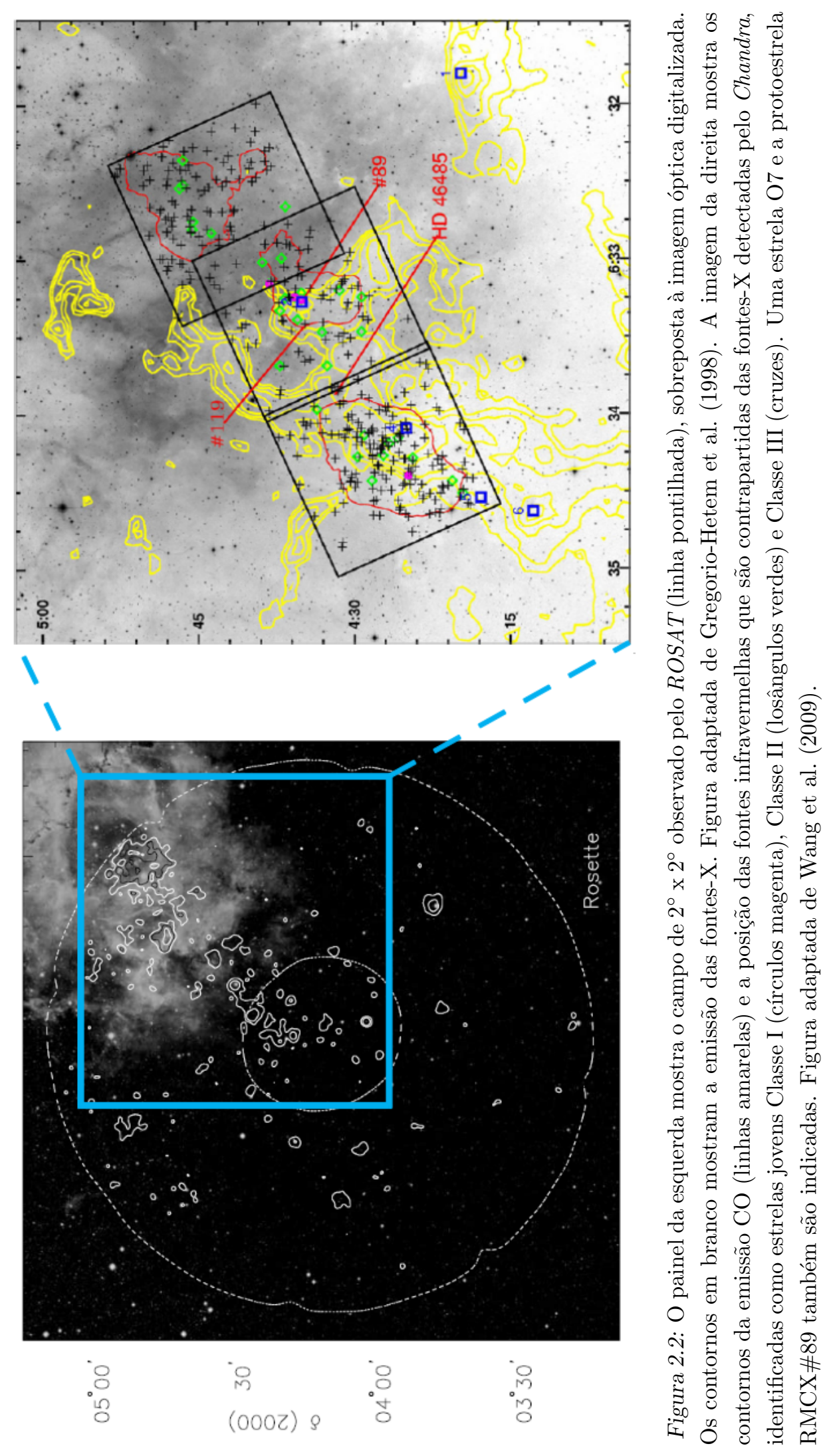




\subsection{Caracterização de aglomerados jovens, com base no infravermelho próximo}

Com a publicação dos catálogos do levantamento All-Sky 2MASS, a região de Rosette foi a primeira a ser completamente coberta espacialmente (Román-Zúñiga e Lada, 2008). Embora o $2 M A S S$ possua uma grande uniformidade em seus dados, sua sensibilidade é baixa. Devido à distância de RMC (1.6 kpc), esse levantamento tem um limite de detecção de $\mathrm{K}=14.3 \mathrm{mag}$.

Li e Smith (2005) estudaram a densidade estelar em Rosette e identificaram duas concentrações principais, correspondentes ao aglomerado NGC2244 e à parte mais densa de RMC. Ao analisar a distribuição de fontes com excesso na cor $H-K$ na nuvem, torna-se evidente uma estrutura filamentária em grande escala. Aglomerados embebidos e estrelas massivas apresentam-se associados espacialmente à essa estrutura. Os candidatos a aglomerados embebidos estão divididos nas sub-regiões A, B, C e D. As características das sub-regiões estão listadas na Tabela 2.4.

A Figura 2.3 mostra a clara correspondência entre as sub-regiões e a distribuição das fontes IRAS mais luminosas. Outro ponto a ser ressaltado é que ambas componentes do sistema binário AFGL 961 são identificadas como candidatas a protoestrelas do tipo O. A componente a leste de AFGL 961 tem massa estimada em $\sim 130 \mathrm{M}_{\odot}$ - sendo provavelmente uma das protoestrelas em formação mais massivas no complexo. Uma estrela do tipo O pode vir a ser uma terceira componente do sistema, devido a sua proximidade com AFGL 961. 
Tabela 2.4 - Aglomerados de fontes detectadas no infrevarmelho próximo (2MASS), localizadas na RMC. Tabela baseada em Li e Smith (2005).

\begin{tabular}{|c|c|c|c|c|c|c|c|c|}
\hline \multirow{3}{*}{$\begin{array}{c}\text { Região } \\
\text { da amostra }\end{array}$} & \multicolumn{4}{|c|}{ Coordenadas Centrais } & \multirow{3}{*}{$\begin{array}{c}\text { Escala } \\
\text { espacial } \\
(\text { arcmin) }\end{array}$} & \multirow{3}{*}{$\begin{array}{c}A_{V} \\
(\mathrm{mag})\end{array}$} & \multirow{3}{*}{$\begin{array}{l}\text { População } \\
\text { embebida }\end{array}$} & \multirow{3}{*}{$\begin{array}{l}\text { Massa do objeto } \\
\text { mais massivo } \\
\qquad\left(\mathrm{M}_{\odot}\right)\end{array}$} \\
\hline & \multicolumn{2}{|c|}{ Galácticas } & \multicolumn{2}{|c|}{ Equatoriais (J2000) } & & & & \\
\hline & $1\left(^{\circ}\right)$ & $\mathrm{b}\left({ }^{\circ}\right)$ & A.R. (h m s) & $\operatorname{Dec}\left({ }^{\circ}, "\right)$ & & & & \\
\hline $\mathrm{A}$ & 203.8079 & -02.4580 & $06: 31: 28.20$ & 04:19:06.6 & $27.90 \times 15.78$ & $0.2-15.0$ & 41 & 20 \\
\hline $\mathrm{B}$ & 206.7739 & -01.9602 & $06: 33: 10.80$ & $04: 34: 40.8$ & $7.20 \times 19.40$ & $0.5-19.5$ & 47 & 20 \\
\hline $\mathrm{C}$ & 207.1228 & -01.8243 & $06: 34: 18.48$ & $04: 19: 51.6$ & $32.00 \times 32.00$ & $0.5-21.5$ & 123 & 130 \\
\hline $\mathrm{D}$ & 206.9162 & -01.8528 & $06: 33: 49.50$ & $04: 30: 04.5$ & $74.25 \times 38.33$ & $0.5-24.5$ & 251 & 50 \\
\hline
\end{tabular}

No levantamento de Wang et al. (2008) foram detectadas 919 fontes de raios-X, sendo que $77 \%$ delas possuem contrapartidas no óptico ou infravermelho próximo (identificadas pelo FLAMINGOS). A população selecionada nesse levantamento tem massa estimada entre 0.5 e $3 M_{\odot}$, e a distribuição espacial dessas fontes está altamente concentrada nas vizinhanças da estrela central do tipo O5, HD 46150. O excesso na banda K é associado à presença de poeira circunstelar e é frequentemente utilizado para identificar estrelas jovens. Neste caso a emissão do disco é proveniente da região mais quente ( $\mathrm{T} \sim 1200 \mathrm{~K}$ ), ou seja, da região mais próxima da estrela.

A Figura 2.4 mostra a distribuição de densidade superficial de estrelas candidatas a contrapartidas infravermelhas (com base nos dados JHK) das fontes detectadas pelo Chandra e estudadas por Wang et al. (2009). Um detalhamento da região em torno da protoestrela RMCX\#89 é apresentado na forma de imagem combinada em três cores com dados Spitzer/IRAC+MIPS, indicando-se as categorias de estrelas jovens associadas. A estrela localiza-se dentro de uma região brilhante de poeira aquecida, rodeada por um conjunto de filamentos infravermelhos que se prolongam por vários minutos de arco, e provavelmente está associada com a região maior de fotodissociação em Rosette. 


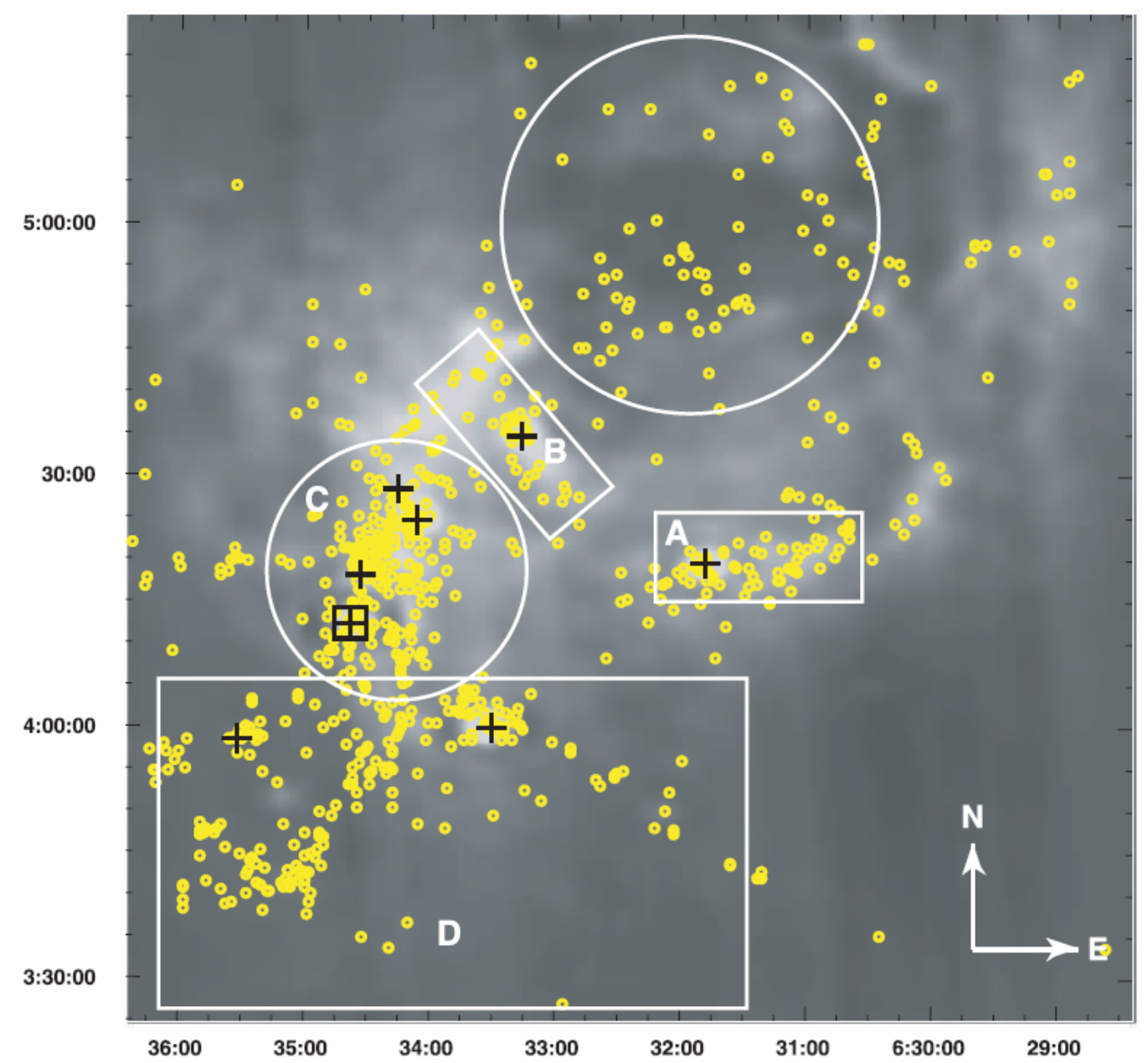

Figura 2.3: Distribuição espacial das fontes $2 M A S S$ com $H-K>0.7$ na RMC, com um campo de $2^{\circ} \times 2^{\circ}$ centrado em $\alpha=06^{h} 32^{m} 24^{s}, \delta=+04^{\circ} 25^{\prime} 00^{\prime \prime}$ (J2000). Regiões bem confinadas de formação de aglomerados estelares estão marcadas como sub-regiões, nomeadas como A, B, C e D. As posições dos sete aglomerados estelares, identificados por Phelps e Lada (1997), estão representados por cruz. A localização das estrelas candidatas a protoestrelas tipo O, associadas a AFGL 961, está indicada por um quadrado em preto. Um círculo na parte superior direita da imagem indica a localização do aglomerado aberto jovem NGC2244 e sua região HII associada. Figura de Li e Smith (2005).

\subsection{Distribuição de gás e poeira: mapas de $C O$ e observações IRAS}

A distribuição de gás molecular em RMC foi estudada em CO por Blitz e Thaddeus (1980) e foi re-analizada por Williams et al. (1995) por meio do algoritmo CLUMPFIND. As estruturas, contendo gás moderadamente denso, $n_{H_{2}} \simeq 10^{3} \mathrm{~cm}^{-3}$, são traçadas pelas linhas $\mathrm{J}=1-0$ de ${ }^{12} \mathrm{CO}$ e ${ }^{13} \mathrm{CO}$. Nas observações de traçadores de baixas densidades, especialmente o $\mathrm{CO}$ e seus isótopos, fornecem informações a respeito da estrutura da 


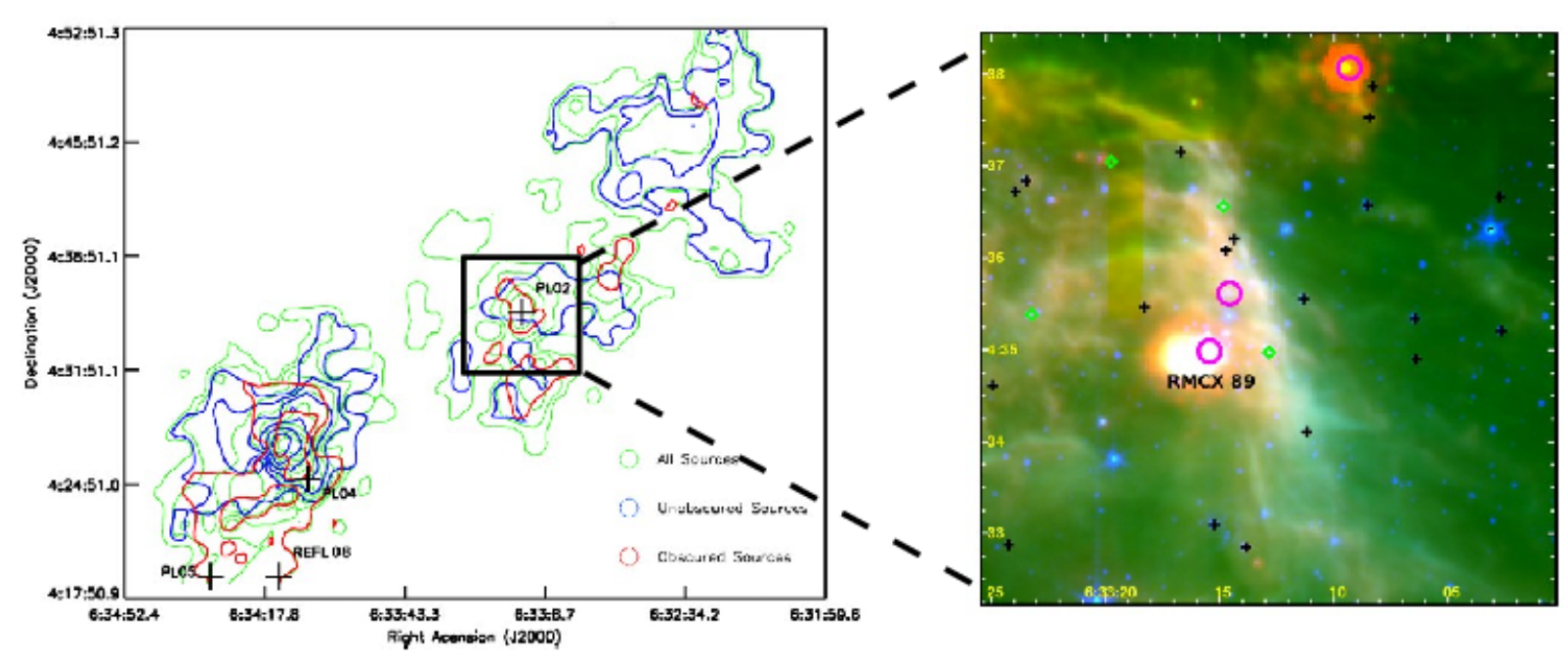

Figura 2.4: O painel da esquerda apresenta a densidade estelar superficial na região de RMC, destacando o aglomerado PL02, que abriga a fonte IRAS 06306+0437 = RMCX \#89. O painel da direita mostra, em zoom, a vizinhança da fonte RMCX \#89 e 25 estrelas com emissão em raio-X no raio de 1 pc. A composição RGB das bandas do Spitzer representam, respectivamente, emissão em 24, 8 e $3.6 \mu$ m. Figuras de Wang et al. (2009).

nuvem molecular. A emissão de ${ }^{12} \mathrm{CO}$ mostra menos contraste do que a emissão de ${ }^{13} \mathrm{CO}$, pois o ${ }^{12} \mathrm{CO}$ tem maior profundidade óptica, enquanto o ${ }^{13} \mathrm{CO}$ é opticamente fino na região de interesse. O estudo de Williams et al. (1995) confirmou a presença de diversos clumps gravitacionalmente ligados. Suas características estão listadas na Tabela 2.5. 
Tabela 2.5 - Lista dos clumps em RMC e suas propriedades, deduzidas da combinação de observações ${ }^{12} \mathrm{CO}$ e ${ }^{13} \mathrm{CO}$. Tabela baseada em Williams et al. (1995).

\begin{tabular}{|c|c|c|c|c|c|c|c|c|c|c|}
\hline Clump & $\begin{array}{l}1 \text { pico } \\
\left({ }^{\circ}\right)\end{array}$ & $\begin{array}{c}\mathrm{b} \text { pico } \\
\left({ }^{\circ}\right)\end{array}$ & $\begin{array}{c}\text { A.R. }(\mathrm{J} 2000) \\
(\mathrm{h} \mathrm{m} \mathrm{s})\end{array}$ & $\begin{array}{c}\operatorname{Dec}(\mathrm{J} 2000) \\
\left({ }^{\circ}, "\right)\end{array}$ & $\begin{array}{c}\mathrm{v}_{\text {pico }} \\
\left(\mathrm{km} \cdot \mathrm{s}^{-1}\right)\end{array}$ & $\begin{array}{l}\mathrm{T}_{e x} \\
(\mathrm{~K})\end{array}$ & $\begin{array}{l}\Delta \mathrm{R} \\
(\mathrm{pc})\end{array}$ & $\begin{array}{c}\Delta \mathrm{v} \\
\left(\mathrm{km} \cdot \mathrm{s}^{-1}\right)\end{array}$ & $\begin{array}{c}\mathrm{M}_{L T E} \\
\left(\mathrm{M}_{\odot}\right)\end{array}$ & $\begin{array}{r}\mathrm{M}_{\text {grav }} \\
\left(\mathrm{M}_{\odot}\right)\end{array}$ \\
\hline 1 & 207.000 & -1.823 & $06: 34: 05.12$ & $04: 26: 26.10$ & 15.6 & 30.9 & 2.36 & 2.13 & 2532 & 674 \\
\hline 2 & 207.250 & -1.823 & $06: 34: 32.78$ & 04:13:07.30 & 12.9 & 8.3 & 3.06 & 2.46 & 2417 & 1160 \\
\hline 3 & 207.550 & -1.723 & $06: 35: 27.31$ & $03: 59: 54.10$ & 12.9 & 11.7 & 4.21 & 2.44 & 2373 & 1572 \\
\hline 4 & 207.775 & -1.773 & $06: 35: 41.48$ & $03: 46: 32.20$ & 11.5 & 8.3 & 3.77 & 1.74 & 2035 & 723 \\
\hline 5 & 207.700 & -1.923 & $06: 35: 01.15$ & 03:46:23.90 & 14.9 & 9.4 & 3.60 & 2.11 & 1700 & 1011 \\
\hline 6 & 207.100 & -1.848 & $06: 34: 10.84$ & $04: 20: 25.20$ & 10.8 & 9.9 & 2.82 & 1.93 & 1540 & 661 \\
\hline 7 & 207.275 & -2.148 & $06: 33: 26.11$ & 04:02:49.30 & 15.6 & 20.3 & 2.79 & 1.59 & 1175 & 444 \\
\hline 8 & 207.125 & -1.898 & 06:34:02.92 & $04: 17: 42.50$ & 12.9 & 11.3 & 1.90 & 1.86 & 1059 & 412 \\
\hline 9 & 207.350 & -1.898 & $06: 34: 27.81$ & 04:05:43.70 & 12.2 & 10.4 & 2.96 & 2.04 & 955 & 780 \\
\hline 10 & 206.825 & -1.998 & 06:33:08.34 & 04:30:55.10 & 16.3 & 19.8 & 1.94 & 2.21 & 934 & 598 \\
\hline 11 & 206.850 & -2.373 & $06: 31: 50.99$ & $04: 19: 13.40$ & 14.2 & 16.8 & 2.21 & 2.08 & 847 & 602 \\
\hline 12 & 207.350 & -1.423 & $06: 36: 09.34$ & $04: 18: 49.50$ & 12.2 & 11.4 & 2.64 & 2.11 & 727 & 738 \\
\hline 13 & 206.875 & -1.898 & $06: 33: 35.25$ & 04:31:01.10 & 14.9 & 19.6 & 1.99 & 2.40 & 701 & 721 \\
\hline 14 & 207.075 & -1.873 & $06: 34: 02.73$ & 04:21:03.70 & 12.9 & 10.5 & 1.98 & 2.18 & 657 & 595 \\
\hline 15 & 207.500 & -2.048 & $06: 34: 12.35$ & 03:53:36.10 & 10.2 & 9.3 & 3.01 & 2.68 & 652 & 1364 \\
\hline 16 & 207.400 & -1.948 & $06: 34: 22.66$ & 04:01:41.10 & 16.3 & 15.8 & 2.31 & 1.64 & 526 & 390 \\
\hline 17 & 207.100 & -1.873 & 06:34:05.50 & $04: 19: 43.80$ & 16.3 & 17.4 & 1.95 & 1.71 & 467 & 359 \\
\hline 18 & 206.775 & -1.948 & $06: 33: 13.49$ & $04: 34: 57.70$ & 15.6 & 18.5 & 1.33 & 1.74 & 452 & 252 \\
\hline 19 & 207.150 & -1.798 & $06: 34: 27.06$ & 04:19:08.20 & 12.2 & 10.3 & 1.50 & 1.73 & 407 & 282 \\
\hline 20 & 207.225 & -1.573 & $06: 35: 23.45$ & $04: 21: 20.90$ & 11.5 & 8.6 & 2.23 & 2.62 & 372 & 967 \\
\hline 21 & 207.675 & -1.923 & $06: 34: 58.39$ & $03: 47: 43.80$ & 11.5 & 9.1 & 2.09 & 1.41 & 337 & 263 \\
\hline 22 & 206.775 & -1.773 & $06: 33: 50.89$ & $04: 39: 47.70$ & 12.9 & 17.3 & 1.40 & 2.25 & 333 & 444 \\
\hline 23 & 207.650 & -1.573 & $06: 36: 10.41$ & $03: 58: 42.40$ & 14.9 & 10.5 & 1.89 & 1.67 & 298 & 333 \\
\hline 24 & 207.900 & -1.798 & $06: 35: 49.93$ & 03:39:11.30 & 10.8 & 9.0 & 1.93 & 1.46 & 294 & 260 \\
\hline 25 & 207.600 & -1.948 & $06: 34: 44.76$ & 03:51:02.10 & 11.5 & 9.1 & 1.85 & 1.80 & 290 & 378 \\
\hline 26 & 207.800 & -1.773 & $06: 35: 44.24$ & $03: 45: 12.30$ & 14.9 & 9.4 & 2.17 & 1.53 & 221 & 318 \\
\hline 27 & 207.850 & -1.598 & $06: 36: 27.15$ & $03: 47: 21.70$ & 10.8 & 7.0 & 2.25 & 2.70 & 211 & 1034 \\
\hline 28 & 207.600 & -1.898 & $06: 34: 55.44$ & $03: 52: 24.80$ & 15.6 & 9.3 & 1.52 & 2.08 & 173 & 414 \\
\hline 29 & 206.800 & -2.523 & $06: 31: 13.41$ & 04:17:44.10 & 13.6 & 8.9 & 1.42 & 1.30 & 164 & 151 \\
\hline 30 & 206.975 & -2.498 & $06: 31: 38.14$ & 04:09:07.10 & 11.5 & 8.9 & 1.30 & 1.48 & 156 & 260 \\
\hline 31 & 207.250 & -2.523 & $06: 32: 03.25$ & 03:53:47.70 & 16.3 & 12.3 & 1.24 & 1.29 & 152 & 130 \\
\hline 32 & 206.925 & -1.648 & $06: 34: 34.23$ & $04: 35: 15.50$ & 14.9 & 13.9 & 1.31 & 1.79 & 150 & 263 \\
\hline 33 & 207.325 & -2.048 & $06: 33: 53.00$ & $04: 02: 55.20$ & 8.1 & 7.0 & 2.00 & 1.45 & 143 & 264 \\
\hline 34 & 207.375 & -1.273 & $06: 36: 44.17$ & $04: 21: 37.50$ & 14.2 & 8.7 & 1.54 & 1.53 & 117 & 226 \\
\hline 35 & 207.900 & -2.048 & $06: 34: 56.53$ & 03:32:18.00 & 15.6 & 8.8 & 1.55 & 1.35 & 103 & 178 \\
\hline 36 & 206.825 & -2.073 & $06: 32: 52.31$ & $04: 28: 50.80$ & 14.2 & 8.6 & 1.49 & 2.25 & 94 & 478 \\
\hline 37 & 207.425 & -1.373 & $06: 36: 28.31$ & $04: 16: 12.40$ & 6.8 & 8.6 & 1.71 & 1.66 & 94 & 296 \\
\hline 38 & 206.950 & -2.073 & $06: 33: 06.16$ & $04: 22: 11.60$ & 19.0 & 9.6 & 1.45 & 3.28 & 84 & 984 \\
\hline 39 & 206.925 & -1.598 & $06: 34: 44.92$ & $04: 36: 38.30$ & 14.2 & 13.9 & 0.24 & 1.41 & 81 & 68 \\
\hline 40 & 207.475 & -1.723 & 06:35:19.02 & $04: 03: 53.80$ & 5.4 & 8.8 & 1.08 & 1.43 & 72 & 138 \\
\hline 41 & 206.775 & -2.498 & $06: 31: 15.98$ & $04: 19: 45.40$ & 11.5 & 9.5 & 1.14 & 1.35 & 68 & 131 \\
\hline 42 & 207.500 & -1.348 & $06: 36: 41.94$ & $04: 12: 53.90$ & 12.9 & 7.7 & 1.40 & 1.98 & 63 & 346 \\
\hline 43 & 207.400 & -1.548 & $06: 35: 48.14$ & $04: 12: 42.90$ & 7.4 & 5.7 & 1.43 & 2.44 & 62 & 538 \\
\hline 44 & 207.300 & -1.073 & $06: 37: 18.66$ & $04: 31: 07.80$ & 10.2 & 7.4 & 1.02 & 1.82 & 62 & 213 \\
\hline 45 & 207.275 & -1.723 & 06:34:56.92 & 04:14:32.90 & 10.2 & 6.7 & 1.02 & 1.91 & 60 & 236 \\
\hline 46 & 207.750 & -2.198 & $06: 34: 07.93$ & 03:36:09.20 & 11.5 & 8.2 & 1.09 & 2.10 & 58 & 300 \\
\hline 47 & 206.900 & -2.248 & $06: 32: 23.23$ & 04:20:01.10 & 12.9 & 11.0 & 1.14 & 1.57 & 58 & 176 \\
\hline 48 & 207.275 & -1.223 & $06: 36: 43.81$ & $04: 28: 19.90$ & 10.2 & 8.5 & 0.74 & 1.40 & 58 & 92 \\
\hline 49 & 207.825 & -1.698 & $06: 36: 03.02$ & $03: 45: 56.40$ & 14.9 & 7.9 & 1.34 & 1.53 & 56 & 199 \\
\hline 50 & 207.100 & -1.648 & $06: 34: 53.59$ & $04: 25: 56.30$ & 9.5 & 8.2 & 0.80 & 1.41 & 56 & 99 \\
\hline 51 & 207.250 & -2.448 & $06: 32: 19.26$ & $03: 55: 52.10$ & 17.0 & 10.5 & 0.83 & 1.05 & 55 & 58 \\
\hline 52 & 208.175 & -1.973 & $06: 35: 42.89$ & $03: 19: 43.10$ & 10.2 & 7.2 & 1.57 & 1.04 & 53 & 108 \\
\hline 53 & 207.500 & -1.873 & $06: 34: 49.73$ & $03: 58: 25.70$ & 10.8 & 6.0 & 0.76 & 3.02 & 43 & 436 \\
\hline 54 & 207.475 & -1.873 & $06: 34: 46.97$ & $03: 59: 45.60$ & 6.8 & 6.5 & 1.11 & 1.42 & 41 & 140 \\
\hline
\end{tabular}


Tabela 2.5 (Continuação)

\begin{tabular}{|c|c|c|c|c|c|c|c|c|c|c|}
\hline Clump & $\begin{array}{l}1 \text { pico } \\
\left({ }^{\circ}\right)\end{array}$ & $\begin{array}{l}\mathrm{b} \text { pico } \\
\left({ }^{\circ}\right)\end{array}$ & $\begin{array}{l}\text { A.R. (J2000) } \\
\quad(\mathrm{h} \mathrm{m} \mathrm{s})\end{array}$ & $\begin{array}{c}\operatorname{Dec}(\mathrm{J} 2000) \\
\left({ }^{\circ}, "\right)\end{array}$ & $\begin{array}{c}\mathrm{v}_{\text {pico }} \\
\left(\mathrm{km} \cdot \mathrm{s}^{-1}\right)\end{array}$ & $\begin{array}{l}\mathrm{T}_{e x} \\
(\mathrm{~K})\end{array}$ & $\begin{array}{l}\Delta \mathrm{R} \\
(\mathrm{pc})\end{array}$ & $\begin{array}{c}\Delta \mathrm{v} \\
\left(\mathrm{km} \cdot \mathrm{s}^{-1}\right)\end{array}$ & $\begin{array}{r}\mathrm{M}_{L T E} \\
\left(\mathrm{M}_{\odot}\right)\end{array}$ & $\begin{array}{r}\mathrm{M}_{\text {grav }} \\
\left(\mathrm{M}_{\odot}\right)\end{array}$ \\
\hline 55 & 206.950 & -2.298 & $06: 32: 18.09$ & $04: 15: 58.60$ & 17.0 & 12.5 & 1.11 & 1.00 & 41 & 70 \\
\hline 56 & 206.925 & -2.423 & $06: 31: 48.62$ & 04:13:51.10 & 14.2 & 7.5 & 0.76 & 1.40 & 40 & 95 \\
\hline 57 & 207.500 & -1.773 & $06: 35: 11.10$ & 04:01:11.20 & 5.4 & 7.9 & 1.09 & 1.95 & 37 & 261 \\
\hline 58 & 207.450 & -1.273 & $06: 36: 52.45$ & $04: 17: 37.70$ & 13.6 & 8.6 & 0.71 & 1.25 & 34 & 70 \\
\hline 59 & 207.075 & -2.498 & $06: 31: 49.21$ & 04:03:47.90 & 11.5 & 8.8 & 0.87 & 1.23 & 33 & 83 \\
\hline 60 & 207.575 & -2.048 & $06: 34: 20.64$ & 03:49:36.50 & 15.6 & 5.3 & 0.65 & 0.95 & 32 & 37 \\
\hline 61 & 207.300 & -2.098 & $06: 33: 39.55$ & 04:02:52.30 & 12.9 & 5.3 & 0.78 & 0.91 & 28 & 40 \\
\hline 62 & 208.100 & -1.723 & $06: 36: 28.02$ & 03:30:35.90 & 12.9 & 5.0 & 0.70 & 1.25 & 27 & 69 \\
\hline 63 & 207.300 & -1.273 & $06: 36: 35.88$ & $04: 25: 37.30$ & 8.8 & 7.1 & 0.78 & 2.34 & 25 & 269 \\
\hline 64 & 207.350 & -1.673 & $06: 35: 15.89$ & 04:11:56.00 & 9.5 & 7.9 & 0.76 & 2.31 & 254 & 257 \\
\hline 65 & 207.300 & -1.923 & $06: 34: 16.94$ & 04:07:42.00 & 16.3 & 6.8 & 0.52 & 0.93 & 20 & 28 \\
\hline 66 & 207.500 & -1.798 & $06: 35: 05.76$ & $04: 00: 29.80$ & 17.6 & 5.0 & 0.88 & 1.45 & 19 & 117 \\
\hline 67 & 207.825 & -2.073 & $06: 34: 42.91$ & $03: 35: 36.30$ & 12.9 & 8.4 & 0.50 & 0.94 & 19 & 28 \\
\hline 68 & 207.675 & -2.073 & $06: 34: 26.34$ & $03: 43: 35.60$ & 12.9 & 5.4 & 0.69 & 1.28 & 17 & 71 \\
\hline 69 & 207.075 & -2.423 & $06: 32: 05.23$ & $04: 05: 52.20$ & 14.9 & 7.0 & 0.70 & 0.96 & 14 & 41 \\
\hline 70 & 207.450 & -1.523 & $06: 35: 59.01$ & $04: 10: 44.40$ & 15.6 & 7.4 & 0.56 & 0.97 & 13 & 33 \\
\hline
\end{tabular}

Para a nuvem molecular Rosette, Williams et al. (1995) buscaram no Catálogo de Fontes Pontuais IRAS (IRAS PSC) fontes infravermelhas com índices de cor análogos àqueles típicos de regiões de formação estelar obtidos para outras nuvens moleculares previamente estudadas. Para que os clumps pudessem ser considerados objetos pré-estelares, o critério adotado foi que as fontes deveriam apresentar fluxo em $25 \mu \mathrm{m}$ maior do que em $12 \mu \mathrm{m}$, além de ter coordenadas galácticas contidas no intervalo de $205.0^{\circ}<1<208.5^{\circ}$ e $-3.0^{\circ}<\mathrm{b}<-1.0^{\circ}$. A Tabela 2.6 apresenta as fontes IRAS associadas aos clumps de Williams et al. (1995). São listadas a densidade de fluxo infravermelho em cada banda $\left(S_{\nu}\right)$, a luminosidade infravermelha total $\left(\mathrm{L}_{I R} \propto \sum \nu S_{\nu}\right)$ e a luminosidade infravermelha estimada utilizando métodos propostos por Casoli et al. (1986) e Parker (1991), que resultam na Equação 2.1:

$$
L_{I R}\left(L_{\odot}\right)=4.7 \times 10^{-6} D^{2}\left[\frac{S_{\nu}(12 \mu m)}{0.79}+\frac{S_{\nu}(25 \mu m)}{2.0}+\frac{S_{\nu}(60 \mu m)}{3.9}+\frac{S_{\nu}(100 \mu m)}{9.9}\right]
$$

onde $D$ é a distância, em parsec, e $S_{\nu}$ é a densidade de fluxo em cada banda $I R A S$, em Janskys.

A Figura 2.5 esboça a localização destas fontes, sobrepostas ao mapa integrado de $\mathrm{CO}$, onde o símbolo representa a posição da fonte e a área do símbolo é proporcional à luminosidade infravermelha da fonte. 
Tabela 2.6 - Fontes pontuais IRAS (infravermelho distante) associadas com RMC. Tabela baseada em Williams et al. (1995).

\begin{tabular}{|c|c|c|c|c|c|c|c|c|c|c|}
\hline \multirow[b]{2}{*}{ IRAS PSC } & \multirow[b]{2}{*}{$\begin{array}{c}1 \\
\left({ }^{\circ}\right) \\
\end{array}$} & \multirow[b]{2}{*}{$\begin{array}{c}\mathrm{b} \\
\left({ }^{\circ}\right) \\
\end{array}$} & \multirow[b]{2}{*}{$\begin{array}{c}\text { A.R. (J2000) } \\
(\mathrm{hms})\end{array}$} & \multirow[b]{2}{*}{$\begin{array}{c}\text { Dec }(\mathrm{J} 2000) \\
\left({ }^{\circ}, \prime\right)\end{array}$} & \multicolumn{4}{|c|}{$S_{\nu}$} & \multirow[b]{2}{*}{$\begin{array}{l}\mathrm{L}_{I R}{ }^{1} \\
(L \odot) \\
\end{array}$} & \multirow[b]{2}{*}{$\begin{array}{c}\mathrm{L}_{I R} \\
\left(L_{\odot}\right)\end{array}$} \\
\hline & & & & & $\begin{array}{c}12 \mu \mathrm{m} \\
\mathrm{Jy}\end{array}$ & $\begin{array}{c}25 \mu \mathrm{m} \\
\mathrm{Jy}\end{array}$ & $\begin{array}{c}60 \mu \mathrm{m} \\
\mathrm{Jy}\end{array}$ & $\begin{array}{c}100 \mu \mathrm{m} \\
\mathrm{Jy}\end{array}$ & & \\
\hline $06319+0415$ & 207.27 & -1.81 & $06: 31: 59.0$ & $+04: 15: 09$ & 78.44 & 375.20 & 958.80 & 995.20 & 11000 & 7619 \\
\hline $06308+0402$ & 207.33 & -2.15 & $06: 33: 31.1$ & $+04: 00: 07$ & 13.35 & 38.29 & 602.60 & 949.00 & 5100 & 3446 \\
\hline $06291+0421$ & 206.84 & -2.38 & $06: 31: 48.5$ & $+04: 19: 31$ & 8.44 & 21.45 & 252.60 & 498.80 & 2500 & 1643 \\
\hline $06314+0427$ & 207.02 & -1.83 & $06: 34: 04.9$ & $+04: 25: 00$ & 6.08 & 6.48 & 129.30 & 323.60 & 1400 & 923 \\
\hline $06306+0437$ & 206.78 & -1.94 & $06: 33: 16.2$ & $+04: 34: 53$ & 3.02 & 11.96 & 88.52 & 274.20 & 1100 & 724 \\
\hline $06300+0530$ & 205.93 & -1.66 & $06: 32: 41.4$ & $+05: 27: 48$ & 1.46 & 1.89 & 35.78 & 130.00 & 480 & 301 \\
\hline $06315+0438$ & 206.87 & -1.73 & $06: 34: 10.7$ & $+04: 36: 08$ & 1.07 & 1.49 & 27.34 & 118.30 & 410 & 253 \\
\hline $06299+0530$ & 205.92 & -1.69 & $06: 32: 34.2$ & $+05: 27: 51$ & 1.54 & 2.04 & 30.33 & 95.07 & 380 & 244 \\
\hline $06283+0516$ & 205.93 & -2.14 & $06: 30: 58.4$ & $+05: 14: 23$ & 0.74 & 1.01 & 21.72 & 102.60 & 340 & 209 \\
\hline $06325+0354$ & 207.64 & -1.85 & $06: 35: 09.3$ & $+03: 51: 43$ & 0.72 & 2.50 & 27.26 & 67.01 & 300 & 191 \\
\hline $06294+0352$ & 207.31 & -2.54 & $06: 32: 07.5$ & $+03: 50: 07$ & 1.45 & 4.42 & 30.00 & 43.96 & 290 & 194 \\
\hline $06306+0232$ & 208.63 & -2.90 & $06: 33: 15.8$ & $+02: 30: 22$ & 0.58 & 4.53 & 25.43 & 51.45 & 270 & 177 \\
\hline $06329+0401$ & 207.58 & -1.71 & $06: 35: 32.3$ & $+03: 58: 52$ & 0.40 & 0.96 & 5.00 & 12.26 & 60 & 42 \\
\hline $06343+0425$ & 207.39 & -1.20 & $06: 37: 00.8$ & $+04: 23: 04$ & 0.25 & 1.10 & 4.86 & 7.38 & 50 & 34 \\
\hline
\end{tabular}

(1) Fornecidas por Williams et al. (1995); (2) Calculadas com a Equação 2.1.

Detalhes da distribuição do gás também podem ser vistos na Figura 2.6, que apresenta as observações em ${ }^{12} \mathrm{CO}$ e ${ }^{13} \mathrm{CO}$ obtidas por Heyer et al. (2006).

É possível perceber que existe uma correlação entre a localização das fontes e a presença do gás molecular. As fontes são consideradas, portanto, estrelas em formação, ainda embebidas no clump de origem.

Além da emissão CO, entre as informações traçadoras das condições físicas nos arredores das estrelas jovens com base em rádio-observações, destaca-se ainda a detecção de fontes maser. Como descrito no Apêndice A, o maser de água, em especial, traça estágios primitivos da formação de estrelas massivas. Essa forte emissão está relacionada com a interação entre as estrelas que se encontram em estágios iniciais da formação e o ambiente que as envolvem, o meio circunstelar. A Tabela 2.7 indica os masers de água detectados em RMC, associados com fontes IRAS. Após uma extensa busca nos catálogos de masers, os objetos de nosso interesse foram encontrados nos trabalhos de Codella et al. (1995) e Sunada et al. (2007), sendo que o primeiro é menos sensível, detectando apenas limites superiores nas medidas de fluxo de nossa amostra. 
Tabela 2.7 - Masers de água detectados na nuvem molecular Rosette.

\begin{tabular}{cccccccc}
\hline \hline IRAS PSC & $\begin{array}{c}\mathrm{l} \\
\left({ }^{\circ}\right)\end{array}$ & $\begin{array}{c}\mathrm{b} \\
\left({ }^{\circ}\right)\end{array}$ & $\begin{array}{c}\text { A.R. }(\mathrm{J} 2000) \\
(\mathrm{h} \mathrm{m} \mathrm{s})\end{array}$ & $\begin{array}{c}\text { Dec }(\mathrm{J} 2000) \\
\left({ }^{\circ}, "\right)\end{array}$ & $\begin{array}{c}\mathrm{A}_{V}{ }^{1} \\
(\mathrm{mag})\end{array}$ & $\begin{array}{c}\mathrm{F}_{\text {pico }} \\
(\mathrm{Jy})\end{array}$ & Referência \\
\hline $06298+0430$ & 206.80 & -2.15 & $06: 32: 31.5$ & $+04: 27: 32$ & 2.04 & $<2.1$ & Codella et al. (1995) \\
$06305+0440$ & 206.72 & -1.94 & $06: 33: 09.6$ & $+04: 38: 04$ & 2.07 & $<2.1$ & Codella et al. (1995) \\
$06282+0440$ & 206.46 & -2.44 & $06: 30: 53.5$ & $+04: 38: 04$ & 2.23 & $<1.8$ & Codella et al. (1995) \\
$06297+0501$ & 206.31 & -1.94 & $06: 32: 23.7$ & $+04: 59: 38$ & 1.3 & $<2.4$ & Codella et al. (1995) \\
$06306+0437$ & 206.78 & -1.94 & $06: 33: 16.4$ & $+04: 34: 57$ & 2.07 & 21.42 & Sunada et al. (2007) \\
$06291+0421$ & 206.84 & -2.38 & $06: 31: 48.1$ & $+04: 19: 31$ & 2.56 & 1.49 & Sunada et al. (2007) \\
$06318+0420$ & 207.18 & -1.79 & $06: 34: 32.3$ & $+04: 17: 48$ & 2.63 & 0.38 & Sunada et al. (2007) \\
\hline
\end{tabular}

(1) Medidas obtidas por meio do mapa de Dobashi et al. (2005). 

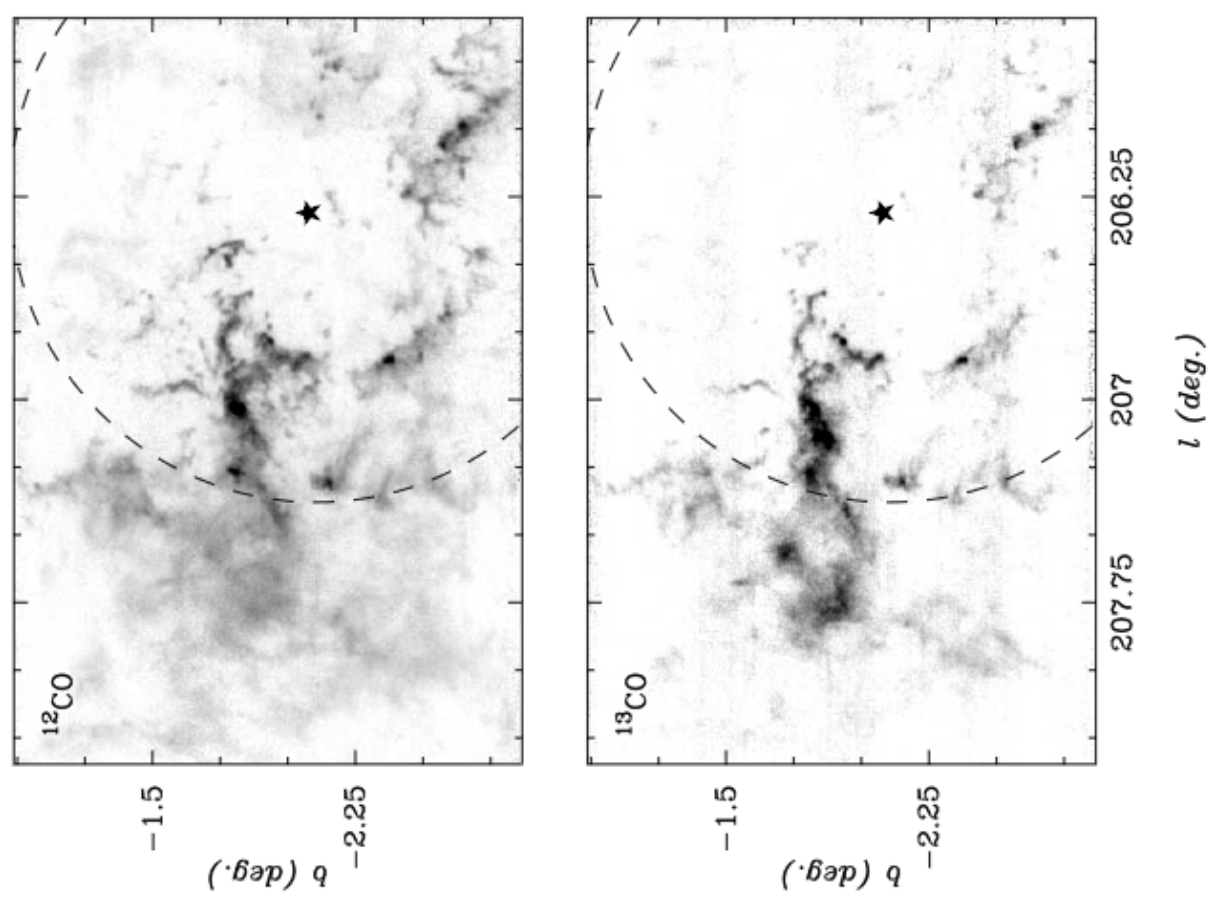

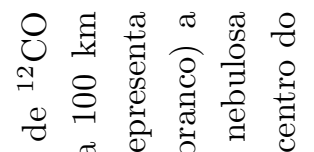

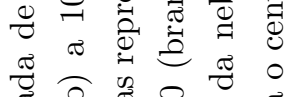

范

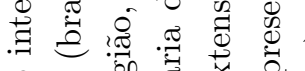

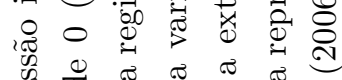

苟

\%

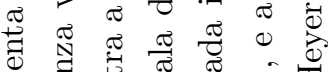

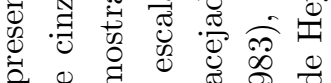

苛

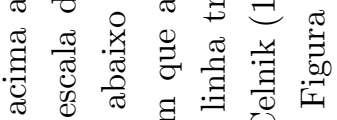

๘

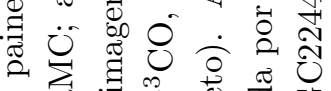

0 业

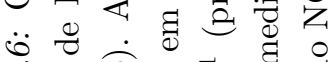

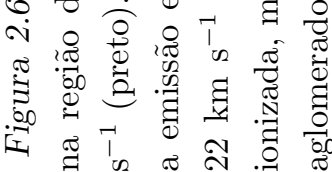

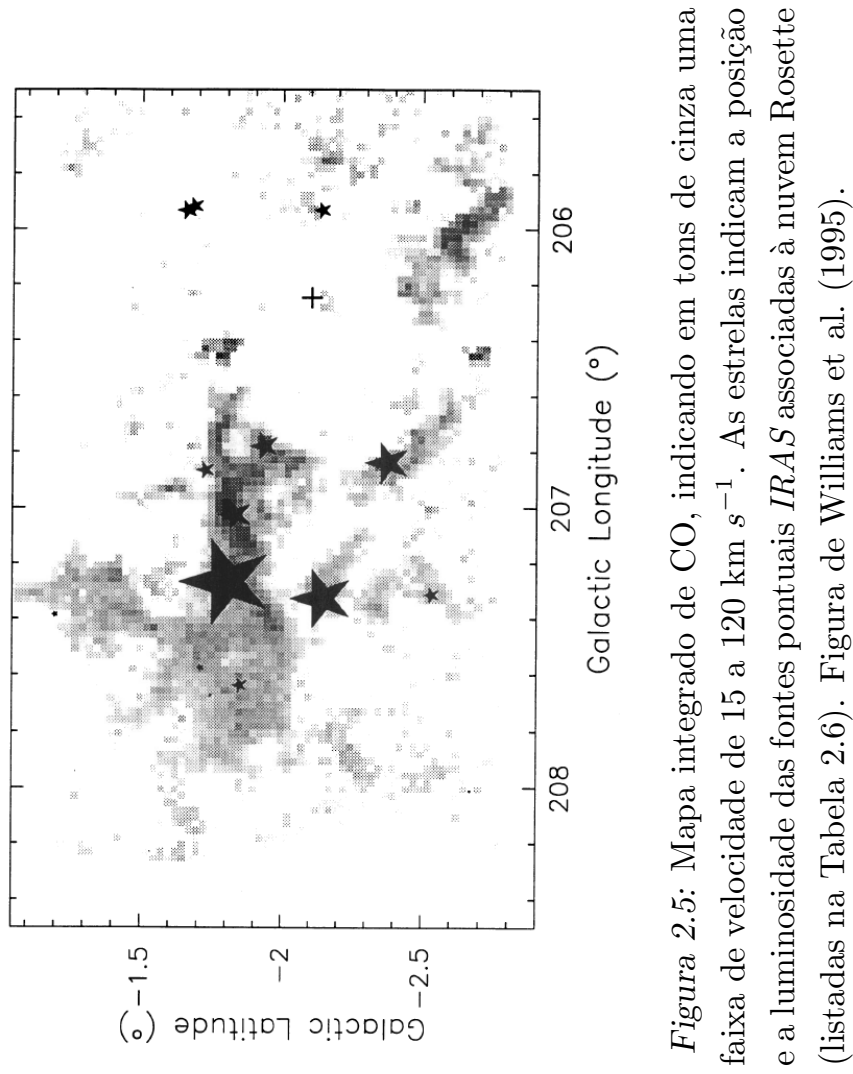


Capítulo 3

\section{A Nuvem Molecular Monoceros (Mon R2)}

Em continuidade ao que foi apresentado para a Nuvem Rosette, este capítulo mostra em detalhe a coleta de informações selecionadas para a Nuvem Molecular Monoceros, a qual costuma ser identificada como Mon R2.

Apresenta-se inicialmente uma descrição do conteúdo estelar e das nebulosas de reflexão, com base nos dados ópticos. Em seguida, discutem-se os resultados obtidos em raios-X, que permitiram ampliar a identificação de estrelas jovens nesta região, da mesma forma que se apresentam os resultados no infravermelho próximo, relacionados à caracterização dos aglomerados estelares associados a Mon R2. Para descrever as características da nuvem, mencionam-se os resultados obtidos no infravermelho distante e os mapeamentos em $\mathrm{CO}$, e finalmente são listadas as fontes maser presentes na região estudada.

\subsection{Identificação óptica de nebulosas de reflexão e aglomerados estelares}

A nuvem molecular Mon R2 localiza-se a cerca de 830 pc de distância (Herbst e Racine, 1976), sua massa é da ordem de $4 \times 10^{4} \mathrm{M}_{\odot}$ (Xie, 1992), estende-se por aproximadamente 88 pc (Carpenter e Hodapp, 2008) e tem idade estimada entre 6 e 10 Myr (Herbst e Racine, 1976). A região Mon R2 é notável por sua cadeia de nebulosas de reflexão iluminadas por estrelas do tipo B. Uma das características interessantes dessa região é estar localizada a uma latitude galáctica relativamente alta (latitudes maiores do que $|\mathrm{b}|=10^{\circ}$ ), o que minimiza a contaminação pelo campo de estrelas (Carpenter, 2000). A Figura 3.1 apresenta a uma visualização das nuvens moleculares ao redor de Mon R2 e foi esquematizada por Maddalena et al. (1986), e a Tabela 3.1 apresenta a lista de estrelas tipo B associadas às nebulosidades, selecionadas de acordo com o trabalho de Carpenter e Hodapp (2008). 


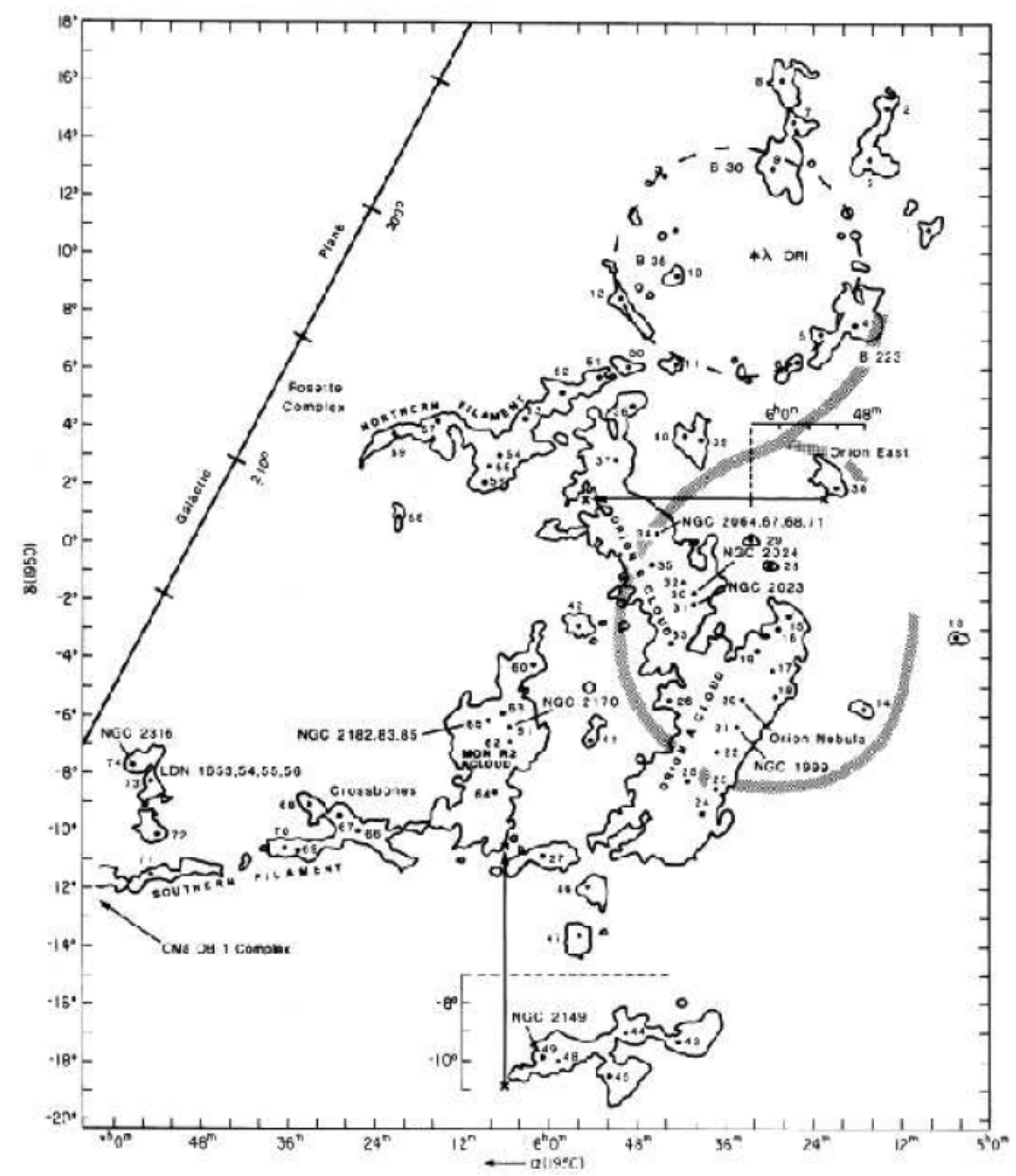

Figura 3.1: Panorama do Complexo de Monoceros e a vizinhança de Mon R2. Contornos indicam a emissão integrada de $\mathrm{CO}$ e os pontos correspondem à posição dos picos de emissão em CO. Figura extraída de Maddalena et al. (1986).

A seleção de objetos jovens associados a Mon R2, com propriedades consistentes com regiões de formação estelar, possibilita a investigação de suas características e permite expandir o conhecimento acerca da evolução de objetos protoestelares nesta nuvem. 
Tabela 3.1 - Estrelas do tipo espectral B, localizadas na nuvem molecular Mon R2. Identificações de Herbst \& Racine (1976). Tabela adaptada de Carpenter e Hodapp (2008).

\begin{tabular}{cccccccc}
\hline \hline ID HR & ID Óptica & $\begin{array}{c}1 \\
\left.{ }^{\circ}\right)\end{array}$ & $\begin{array}{c}\text { b } \\
\left({ }^{\circ}\right)\end{array}$ & $\begin{array}{c}\text { A.R. }(\mathrm{J} 2000) \\
(\mathrm{h} \mathrm{m} \mathrm{s})\end{array}$ & $\begin{array}{c}\text { Dec }(\mathrm{J} 2000) \\
\left({ }^{\circ}, "\right)\end{array}$ & $\begin{array}{c}\text { Tipo Espectral } \\
\mathrm{m}_{V} \\
(\mathrm{mag})\end{array}$ \\
\hline HR 4 & GSC 04795-01779 & 213.4125 & -12.3950 & $06: 07: 32$ & $-6: 24: 03$ & B1 V & 10.4 \\
HR 8b & GSC 04795-01778 & 213.7182 & -12.5233 & $06: 08: 05$ & $-6: 21: 34$ & B2.5 V & 9.63 \\
HR 9b & HD 42004 & 213.5927 & -12.4656 & $06: 08: 04$ & $-6: 13: 38$ & B1.5 V & 9.6 \\
HR 10 & GSC 04791-02696 & 212.8229 & -11.9886 & $06: 08: 26$ & $-5: 20: 19$ & B1 V & 8.12 \\
HR 13 & GSC 04795-01776 & 213.8482 & -12.1883 & $06: 09: 31$ & $-6: 19: 36$ & B3 V & 9.29 \\
HR 14 & GSC 04795-01462 & 213.8585 & -12.1312 & $06: 09: 45$ & $-6: 18: 35$ & B5 V & 10.36 \\
HR 15 & Mon R2-15 & 213.5327 & -12.0417 & $06: 10: 01$ & $-6: 18: 42$ & B8 V & 11.40 \\
HR 22 & GSC 04795-00509 & 213.9221 & -11.7853 & $06: 11: 06$ & $-6: 12: 33$ & B8-A0 & $\ldots$ \\
HR 23 & GSC 04795-00401 & 213.9539 & -11.6035 & $06: 11: 49$ & $-6: 09: 22$ & B4 V & 10.9 \\
HR 27 & Mon R2-27 & 214.5031 & -11.0198 & $06: 14: 53$ & $-6: 22: 44$ & B7 V & 10.98 \\
HR 28 & GSC 04796-01931 & 214.6036 & -10.9378 & $06: 15: 21$ & $-6: 25: 50$ & B5 V & 10.70 \\
\hline
\end{tabular}

A Figura 3.2 mostra a localização das nebulosas de reflexão em Mon R2, extraídas dos catálogos GGD (Gyulbudaghian et al., 1978) e DG (Dorschner e Gürtler, 1963). Também indica-se a posição das estrelas com identificação HR (Herbst e Racine, 1976). Nesta figura, baseada no trabalho de Carpenter e Hodapp (2008), também se apresenta uma imagem óptica colorida, para destacar o core principal de Mon R2, bem como as demais partes da nuvem que aparecem como regiões escuras em contraste com as nebulosas de emissão. 

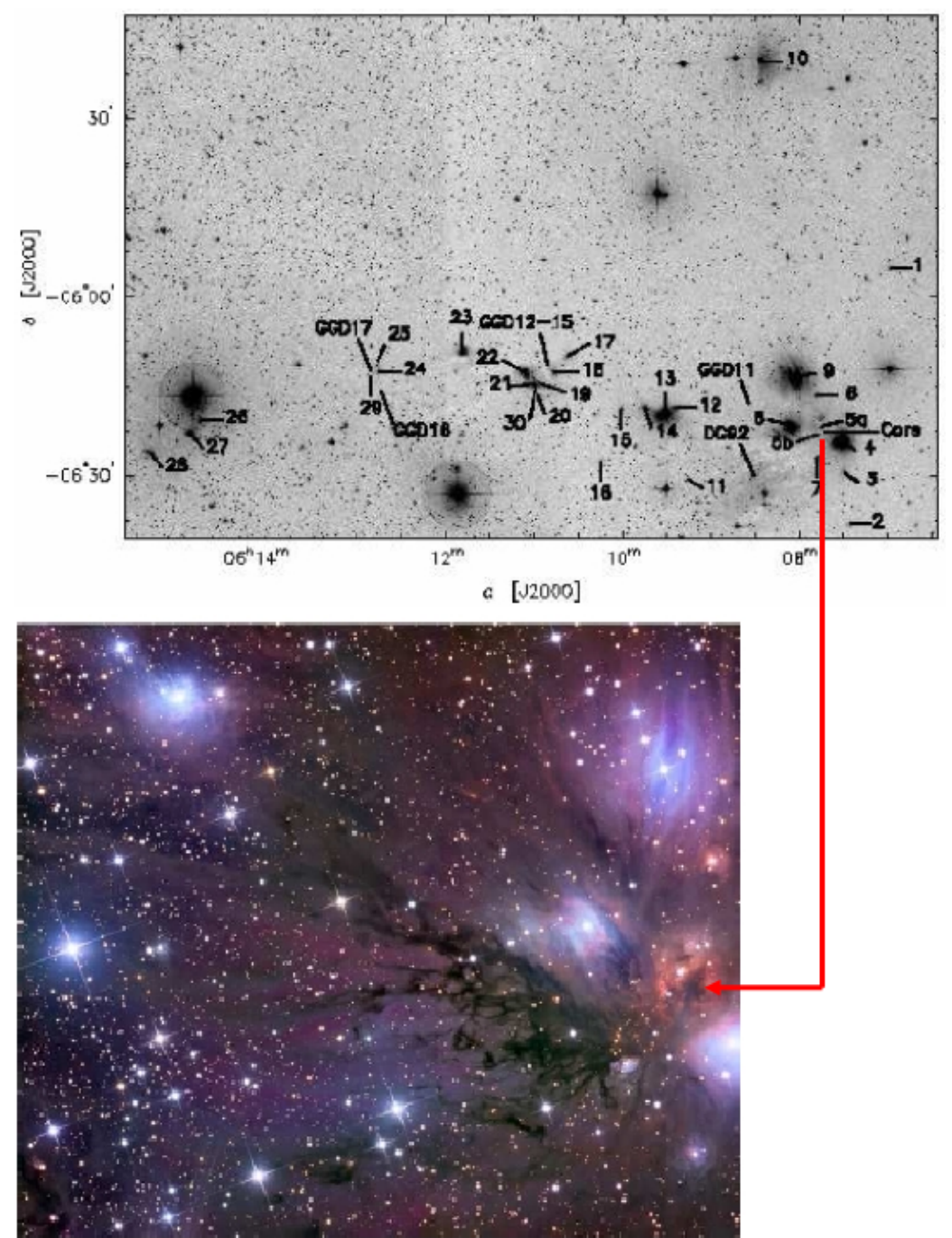

Figura 3.2: No painel superior apresenta-se a imagem óptica (POSS-B) identificando-se as nebulosas ópticas catalogadas por GGD e DG (vide texto) em Mon R2. A posição das estrelas aparece indicada por seu respectivo número HR. A fotografia colorida do painel inferior (cortesia de R. Croman) destaca a posição do core denso de Mon R2 (seta vermelha), bem como as demais nuvens escuras que se encontram na região. Figura adaptada de Carpenter e Hodapp (2008).

\subsection{Detecção de estrelas jovens em Mon $R 2$ por meio de raios- $X$}

O dados do satélite ROSAT permitiram que Gregorio-Hetem et al. (1998) identificassem estrelas jovens de baixa massa em Mon R2. As observações revelaram a presença de dezenas de estrelas $P M S$, e esse estudo mostrou que existe uma correlação entre formação estelar ativa no core de Mon R2 e a presença de fontes de raios-X. Assim como foi feito para a região de Rosette, as observações ROSAT cobriram um campo de $2^{\circ}$ de diâmetro, cen- 
trada no core denso da nuvem, que coincide com a região de alta emissão no infravermelho. Foram detectadas 41 fontes pontuais de raios-X, listadas na Tabela 3.2.

Tabela 3.2 - Fontes pontuais ROSAT associadas a Mon R2. Tabela baseada em Gregorio-Hetem et al. (1998).

\begin{tabular}{|c|c|c|c|c|c|c|c|c|}
\hline $\begin{array}{c}\text { No. } \\
- \\
\end{array}$ & $\begin{array}{c}\text { Nome } \\
\text { RXJ }\end{array}$ & $\begin{array}{c}1 \\
\left({ }^{\circ}\right) \\
\end{array}$ & $\begin{array}{l}\mathrm{b} \\
\left({ }^{\circ}\right) \\
\end{array}$ & $\begin{array}{c}\mathrm{AR} \\
(\mathrm{h} \mathrm{m} \mathrm{s}) \\
\end{array}$ & $\begin{array}{c}\text { Dec } \\
\left({ }^{\circ}, "\right)\end{array}$ & $\begin{array}{c}\text { Cnts/Ks } \\
- \\
\end{array}$ & $\begin{array}{l}\log \mathrm{L}_{X} \\
(\mathrm{erg} / \mathrm{s}) \\
\end{array}$ & $\begin{array}{l}\text { Identificação óptica } \\
\qquad-\end{array}$ \\
\hline 1 & $0605.8-0617$ & 213.59 & -12.98 & $6: 05: 52.7$ & $-6: 17: 03$ & $5.7 \pm 1.0$ & $30.62 \pm 0.10$ & 4794-00481 \\
\hline 2 & $0606.3-0614$ & 213.40 & -12.85 & $6: 06: 20.4$ & $-6: 14: 11$ & $9.0 \pm 1.1$ & $30.82 \pm 0.08$ & 4794-01329 \\
\hline 3 & 0606.6-0631 & 213.70 & -12.93 & $6: 06: 36.3$ & $-6: 31: 41$ & $14.1 \pm 1.4$ & $31.02 \pm 0.07$ & 4794-01261 \\
\hline 4 & 0606.6-0617 & 213.49 & -12.79 & $6: 06: 41.8$ & $-6: 17: 17$ & $3.6 \pm 0.7$ & $30.42 \pm 0.11$ & $\ldots$ \\
\hline 5 & 0606.7-0634 & 213.75 & -12.92 & $6: 06: 42.2$ & $-6: 34: 15$ & $2.4 \pm 0.6$ & $30.25 \pm 0.13$ & $\ldots$ \\
\hline 6 & 0606.7-0649 & 213.99 & -13.02 & $6: 06: 46.1$ & $-6: 49: 42$ & $4.5 \pm 0.8$ & $30.52 \pm 0.10$ & $\ldots$ \\
\hline 7 & 0606.9-0633 & 213.77 & -12.86 & $6: 06: 57.0$ & $-6: 33: 18$ & $1.4 \pm 0.4$ & $30.01 \pm 0.15$ & $\ldots$ \\
\hline 8 & $0607.3-0623$ & 213.66 & -12.71 & $6: 07: 18.7$ & $-6: 23: 41$ & $1.8 \pm 0.5$ & $30.12 \pm 0.14$ & (1) \\
\hline 9 & 0607.3-0615 & 213.54 & -12.65 & $6: 07: 19.7$ & $-6: 15: 39$ & $2.7 \pm 0.6$ & $30.30 \pm 0.12$ & 4794-00213 \\
\hline 10 & 0607.3-0549 & 213.14 & -12.45 & $6: 07: 20.8$ & $-5: 49: 27$ & $11.4 \pm 1.4$ & $30.93 \pm 0.08$ & 4794-00633 \\
\hline 11 & 0607.3-0626 & 213.71 & -12.72 & $6: 07: 21.3$ & $-6: 26: 48$ & $2.2 \pm 0.5$ & $30.21 \pm 0.12$ & $\ldots$ \\
\hline 12 & 0607.4-0638 & 213.89 & -12.79 & $6: 07: 26.5$ & $-6: 38: 06$ & $7.9 \pm 1.0$ & $30.77 \pm 0.08$ & 4794-00775 \\
\hline 13 & 0607.4-0607 & 213.43 & -12.56 & $6: 07: 27.7$ & $-6: 07: 12$ & $1.7 \pm 0.5$ & $30.10 \pm 0.15$ & $\ldots$ \\
\hline 14 & 0607.5-0620 & 213.63 & -12.64 & $6: 07: 30.4$ & $-6: 20: 02$ & $2.0 \pm 0.5$ & $30.17 \pm 0.13$ & $\ldots$ \\
\hline 15 & 0607.5-0621 & 213.66 & -12.65 & $6: 07: 30.7$ & $-6: 21: 56$ & $1.4 \pm 0.4$ & $30.01 \pm 0.15$ & $\ldots$ \\
\hline 16 & 0607.5-0636 & 213.88 & -12.76 & $6: 07: 31.1$ & $-6: 36: 38$ & $1.8 \pm 0.5$ & $30.12 \pm 0.14$ & $\ldots$ \\
\hline 17 & 0607.5-0632 & 213.83 & -12.72 & $6: 07: 33.0$ & $-6: 32: 43$ & $3.5 \pm 0.6$ & $30.41 \pm 0.10$ & $\ldots$ \\
\hline 18 & 0607.6-0626 & 213.75 & -12.65 & $6: 07: 41.1$ & $-6: 26: 57$ & $2.3 \pm 0.5$ & $30.23 \pm 0.12$ & $\ldots$ \\
\hline 19 & 0607.7-0629 & 213.80 & -12.65 & $6: 07: 45.7$ & $-6: 29: 33$ & $2.4 \pm 0.5$ & $30.25 \pm 0.11$ & $\ldots$ \\
\hline 20 & 0607.7-0621 & 213.68 & -12.59 & $6: 07: 45.9$ & $-6: 21: 46$ & $3.0 \pm 0.7$ & $30.35 \pm 0.13$ & $4795-01568$ \\
\hline 21 & 0607.7-0625 & 213.75 & -12.62 & $6: 07: 47.2$ & $-6: 25: 49$ & $1.5 \pm 0.4$ & $30.04 \pm 0.14$ & $\ldots$ \\
\hline 22 & 0607.7-0622 & 213.70 & -12.59 & $6: 07: 47.8$ & $-6: 22: 52$ & $1.6 \pm 0.5$ & $30.08 \pm 0.16$ & 4795-00995 \\
\hline 23 & $0607.8-0635$ & 213.89 & -12.68 & $6: 07: 49.0$ & $-6: 35: 01$ & $2.3 \pm 0.5$ & $30.23 \pm 0.12$ & $\ldots$ \\
\hline 24 & 0607.8-0619 & 213.66 & -12.57 & $6: 07: 49.4$ & $-6: 19: 35$ & $0.8 \pm 0.3$ & $29.77 \pm 0.19$ & $\ldots$ \\
\hline 25 & $0607.8-0625$ & 213.75 & -12.61 & $6: 07: 50.9$ & $-6: 25: 51$ & $2.2 \pm 0.5$ & $30.21 \pm 0.12$ & $\cdots$ \\
\hline 26 & 0607.9-0616 & 213.63 & -12.52 & $6: 07: 55.1$ & $-6: 16: 53$ & $3.5 \pm 0.6$ & $30.41 \pm 0.10$ & $\ldots$ \\
\hline 27 & 0607.9-0635 & 213.91 & -12.66 & $6: 07: 55.7$ & $-6: 35: 05$ & $1.7 \pm 0.4$ & $30.10 \pm 0.13$ & $\ldots$ \\
\hline 28 & 0607.9-0620 & 213.69 & -12.55 & $6: 07: 55.9$ & $-6: 20: 54$ & $1.2 \pm 0.5$ & $29.94 \pm 0.20$ & $\ldots$ \\
\hline 29 & 0608.0-0616 & 213.63 & -12.51 & 6:08:00.1 & $-6: 16: 54$ & $4.2 \pm 0.7$ & $30.49 \pm 0.10$ & $\ldots$ \\
\hline 30 & 0608.0-0632 & 213.87 & -12.62 & $6: 08: 00.7$ & $-6: 32: 22$ & $2.2 \pm 0.5$ & $30.21 \pm 0.12$ & $\ldots$ \\
\hline 31 & 0608.0-0613 & 213.59 & -12.47 & $6: 08: 03.5$ & $-6: 13: 49$ & $1.0 \pm 0.3$ & $29.87 \pm 0.15$ & (2) \\
\hline 32 & 0608.0-0636 & 213.95 & -12.64 & $6: 08: 04.0$ & $-6: 36: 55$ & $0.8 \pm 0.3$ & $29.77 \pm 0.19$ & $\ldots$ \\
\hline 33 & $0608.2-0611$ & 213.58 & -12.41 & $6: 08: 15.2$ & $-6: 11: 32$ & $2.1 \pm 0.5$ & $30.19 \pm 0.13$ & (3) \\
\hline 34 & 0608.4-0631 & 213.91 & -12.53 & $6: 08: 24.1$ & $-6: 31: 36$ & $2.0 \pm 0.5$ & $30.17 \pm 0.13$ & $\cdots$ \\
\hline 35 & 0608.5-0628 & 213.88 & -12.46 & $6: 08: 34.7$ & $-6: 28: 37$ & $2.1 \pm 0.5$ & $30.19 \pm 0.13$ & $\ldots$ \\
\hline 36 & 0608.5-0633 & 213.96 & -12.49 & $6: 08: 35.7$ & $-6: 33: 37$ & $2.9 \pm 0.8$ & $30.33 \pm 0.14$ & $\ldots$ \\
\hline 37 & 0608.6-0610 & 213.61 & -12.31 & $6: 08: 40.5$ & $-6: 10: 29$ & $3.7 \pm 0.9$ & $30.44 \pm 0.13$ & $(4)$ \\
\hline 38 & $0608.7-0638$ & 214.04 & -12.51 & $6: 08: 42.4$ & $-6: 38: 23$ & $3.2 \pm 0.7$ & $30.37 \pm 0.12$ & $4795-01339$ \\
\hline 39 & 0608.7-0649 & 214.22 & -12.57 & $6: 08: 48.2$ & $-6: 49: 32$ & $41.2 \pm 2.5$ & $31.48 \pm 0.05$ & $4795-01744^{(6)}$ \\
\hline 40 & 0609.5-0619 & 213.85 & -12.17 & $6: 09: 34.7$ & $-6: 19: 11$ & $15.9 \pm 1.6$ & $31.07 \pm 0.07$ & $\ldots$ \\
\hline 41 & 0609.5-0544 & 213.31 & -11.91 & 6:09:34.7 & $-5: 44: 06$ & $22.4 \pm 2.1$ & $31.22 \pm 0.07$ & $4795-01090^{(7)}$ \\
\hline
\end{tabular}

A exemplo da Figura 2.2, apresentamos na Figura 3.3 a distribuição das fontes ROSAT sobrepostas à imagem óptica. Os resultados sugerem que a maior parte das fontes detectadas são identificadas como estrelas Herbig Ae/Be e estrelas T Tauri. Emissão extensa e difusa também foi detectada e pode ser devida à contribuição total de várias fontes pun- 
tiformes, tratando-se de emissão não resolvida na nuvem Mon R2. Como objetos estelares jovens de alta massa geralmente estão localizados em cores densos de $G M C$, raios-X moles (associados a energias mais baixas) podem ser fortemente absorvidos, portanto instrumentos de imageamento de raios-X duros (associados a energias mais altas) são essencialmente necessários.

Para comparação com os resultados ROSAT, o painel da direita da Figura 3.3 apresenta uma imagem Chandra obtida por Kohno et al. (2002), na direção de objetos estelares jovens de alta massa localizados na nuvem; foram detectadas 154 fontes, sendo $85 \%$ delas identificadas com contrapartidas infravermelhas.

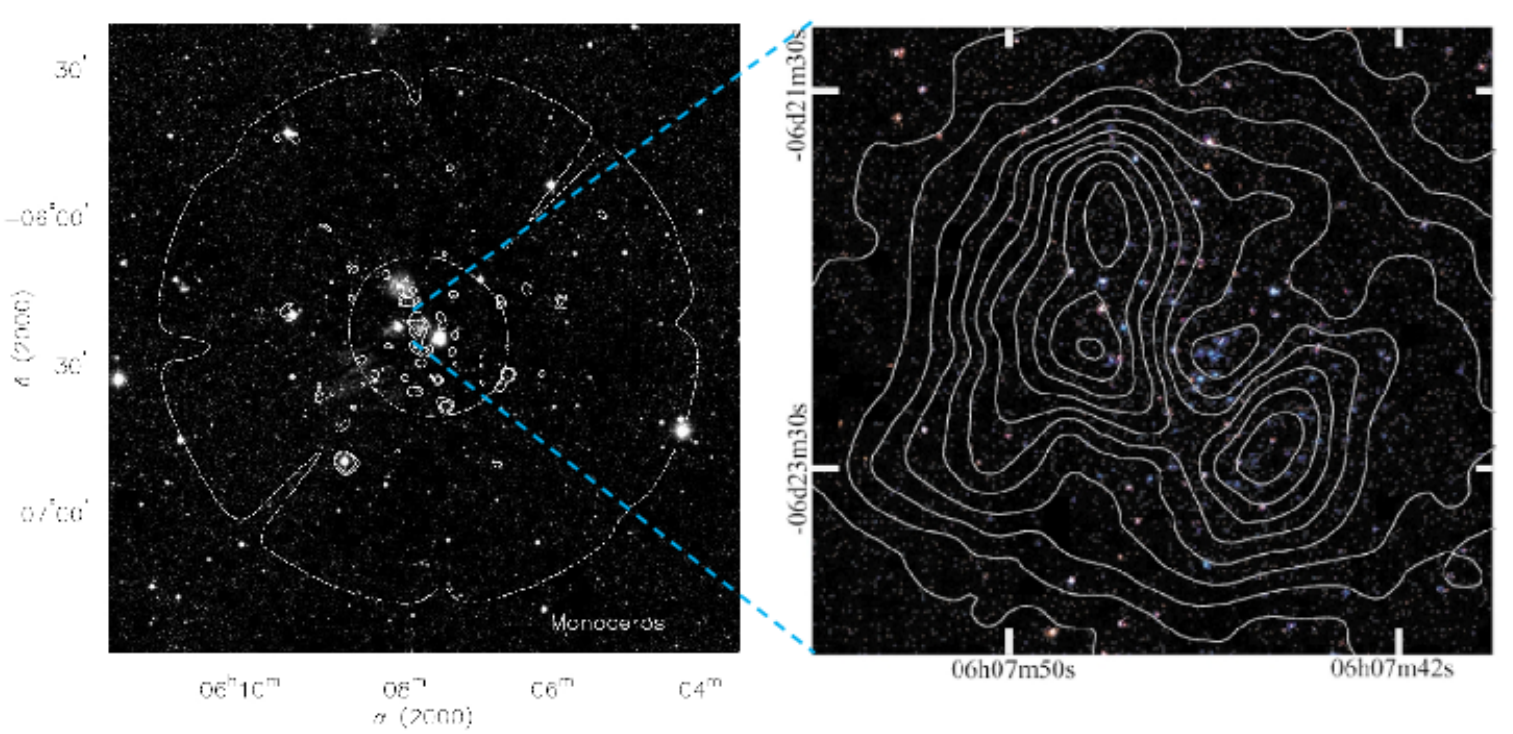

Figura 3.3: O painel da esquerda mostra o campo de $2^{\circ} \mathrm{x} 2^{\circ}$ observado pelo $R O S A T$ (linha branca pontilhada), sobreposta à imagem óptica digitalizada na nuvem de Mon R2. Os contornos em branco mostram a emissão das fontes-X (figura adaptada de Gregorio-Hetem et al. 1998). O painel da direita mostra, em um campo de 3'.2 x 3'.2, contornos de emissão CS (linhas brancas) e a posição das fontes-X detectadas pelo Chandra. Figura adaptada de Kohno et al. (2002).

Posteriormente, Nakajima et al. (2003) também utilizaram o satélite Chandra para estudar a região central de Mon R2 e detectaram 368 fontes de raios-X. Cerca de $80 \%$ dessas fontes foram identificadas com contrapartidas no infravermelho próximo. As magnitudes nas bandas JHK do $2 M A S S$ foram usadas para estimar a fase evolutiva (estrelas T Tauri clássicas e T Tauri de linhas fracas) e a massa das fontes emissoras de raios-X. Além disso, as propriedades dos raios-X foram analisadas como função da idade e da massa da fonte. 


\subsection{Caracterização de aglomerados jovens, com base no infravermelho próximo}

Estrelas de menor massa, contidas na nuvem de Mon R2 foram investigadas por Carpenter (2000), que usou o catálogo de fontes pontuais 2MASS para identificar aglomerados estelares. Os resultados sugerem que uma quantidade considerável das estrelas em Mon R2 está distribuída em apenas dois dos aglomerados, mas uma população isolada também pode existir.

Com base no aumento de densidade superficial de estrelas, relativa à distribuição de estrelas no campo, quatro aglomerados foram encontrados e são mostrados na Figura 3.4, a qual identifica a posição dos aglomerados associados ao core de Mon R2 (que contém 371 estrelas), GGD 12-15 (134 estrelas), GGD 17 (23 estrelas) e IRAS 06046-0603 (15 estrelas). Esta última está associada a um objeto Herbig-Haro (HH 886), cuja presença confirma as condições típicas de estrelas em formação, envolvendo jatos.

Carpenter e Hodapp (2008) estudaram o pequeno grupo de nebulosas de reflexão denominado GGD12-15, que faz parte de um core denso no qual também se encontra uma fonte maser de água, associada a uma região HII. A Figura 3.5 mostra, no painel da esquerda, uma imagem obtida a partir do $P O S S$ e o painel da direita traz uma imagem 2MASS, na banda $\mathrm{K}$, ambas indicando a posição destes interessantes objetos que se encontram próximos a GGD 12-15.

\subsection{Distribuição de gás molecular e associação com fontes IRAS}

De acordo com Carpenter e Hodapp (2008), os primeiros resultados da detecção de CO na região de Mon R2 foram apresentados por Loren et al. (1974) e Kutner e Tucker (1975), este em particular mostrando que pelo menos 5 das nebulosas de reflexão estão associadas com picos nos mapas de ${ }^{12} \mathrm{CO}$. Posteriormente, Maddalena et al. (1986) apresentaram um mapa de $\mathrm{CO}$ em grande escala, abrangendo todo o Complexo Orion-Monoceros, como foi apresentado na Figura 3.1. Trabalhos mais recentes, e com melhores sensibilidade e resolução angular, apresentam imagens da emissão J=1-0 de ${ }^{12} \mathrm{CO}$ (Xie e Goldsmith, 1994) e ${ }^{13} \mathrm{CO}$ (Miesch et al., 1999), que reproduzimos na Figura 3.6.

Para comparação com o infravermelho distante, o painel à direita da Figura 3.6 apre- 


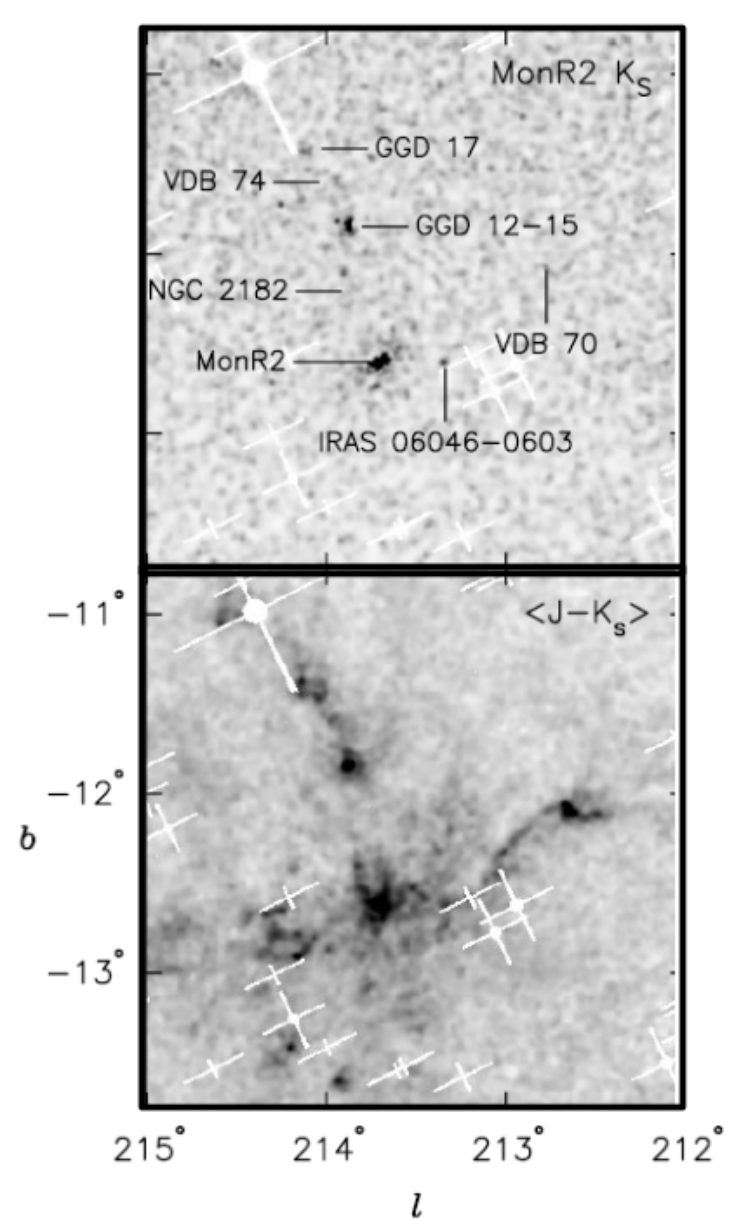

Figura 3.4: O painel acima mostra um mapa de densidade superficial de estrelas na banda $\mathrm{K}$, em Mon R2, para estrelas com magnitudes de $6.0 \mathrm{mag} \leq m(K s) \leq 14.3 \mathrm{mag}$. O painel de baixo traz uma imagem da cor média $\mathrm{J}-\mathrm{K}$ para estrelas observadas pelo $2 M A S S$; as cruzes brancas indicam regiões ao redor de estrelas brilhantes. Os tons mais escuros representam intensidades maiores. Figuras extraídas de Carpenter (2000).

senta a imagem em $60 \mu \mathrm{m}$, no qual se verifica a correspondência da emissão IRAS mais intensa bem correlacionada com os máximos de intensidade da emissão CO (painéis da esquerda), bem como a distribuição do excesso de cor no infravermelho próximo (J-K) mostrada na Figura 3.4, de acordo com Carpenter (2000).

A partir de características relacionadas com o fluxo da emissão em cada banda do infravermelho distante, Xie (1992) selecionou uma amostra de fontes pontuais IRAS (Tabela 3.3), na nuvem Monoceros $R 2$, que possuem $\mathrm{S}_{\nu}(25 \mu \mathrm{m}) \geq \mathrm{S}_{\nu}(60 \mu \mathrm{m})$. Esse estudo forneceu um levantamento sensível das estrelas embebidas contidas em toda a nuvem molecular e comparou a posição das fontes em relação ao gás presente na nuvem. 


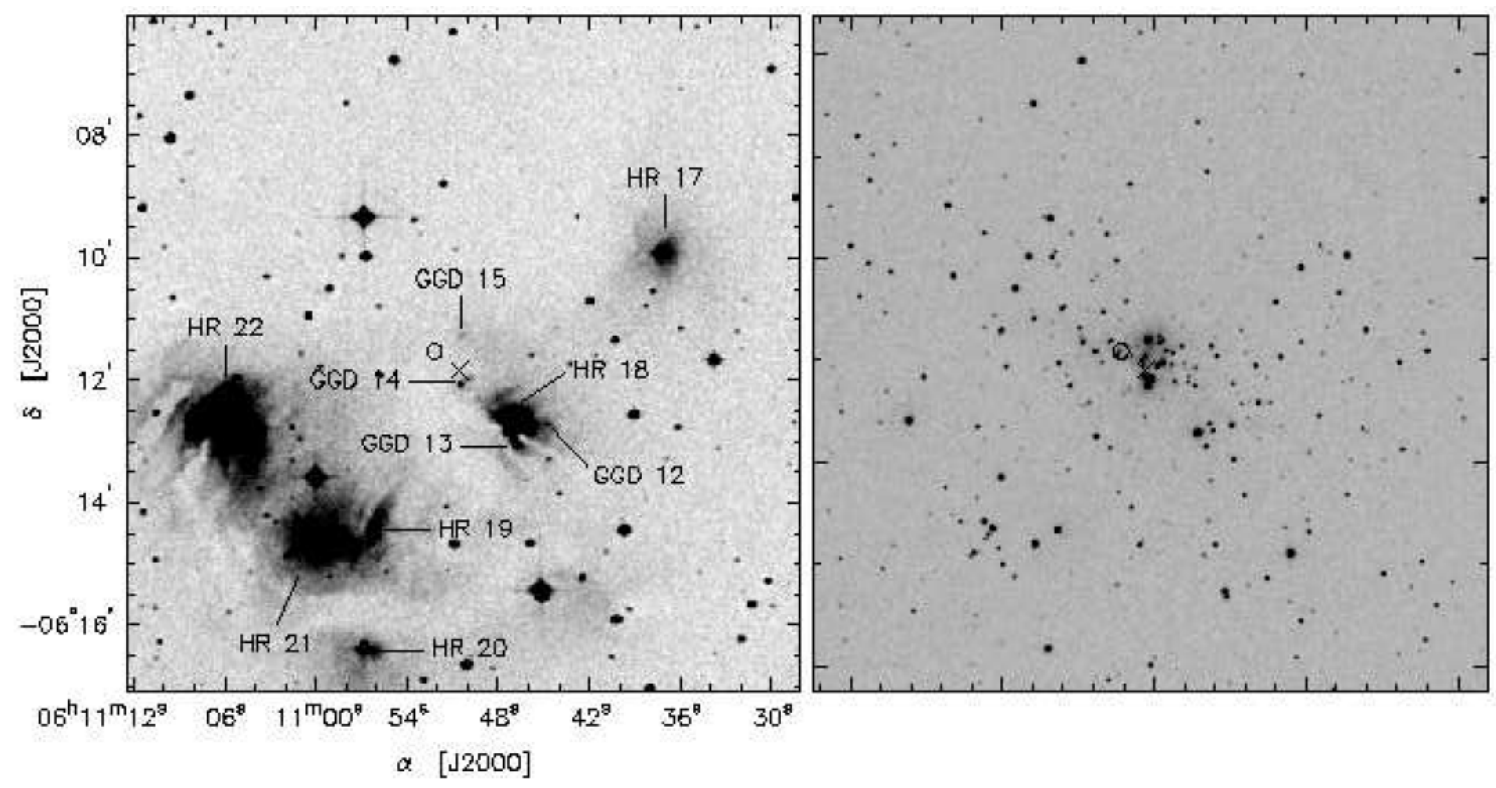

Figura 3.5: Imagens da região GGD 12-15, em Monoceros R2. A cruz indica a posição da região HII e o círculo aberto indica a posição do maser de água, em ambos painéis. Figura de Carpenter e Hodapp (2008).

Dado que altas extinções estão geralmente associadas com regiões de formação estelar profundamente embebidas, podem existir aglomerados ricos ( $\geq 100$ estrelas) que ainda não foram detectados nos comprimentos de onda na faixa do infravermelho. Aglomerados assim podem aparecer em imagens IRAS na banda de $60 \mu \mathrm{m}$, que sondam mais profundamente as nuvens moleculares do que observações na banda K. Em Mon R2, três fontes IRAS brilhantes, correspondentes a nebulosas de reflexão, não possuem aglomerados aparentemente associados, identificados com base nos dados $2 M A S S$.

A amostra selecionada por Xie (1992) contém 36 fontes pontuais IRAS contidas em Mon R2, que possuem propriedades características de objetos estelares jovens. A Tabela 3.3 lista o fluxo infravermelho obtido para cada fonte, nas quatro bandas do $I R A S$, e a luminosidade infravermelha total, calculada por meio da Equação 2.1. Nessa amostra, as luminosidades $I R A S$ variam entre $1.5 L_{\odot}$ e 26,000 $L_{\odot}$. É interessante ressaltar que as duas fontes IRAS mais luminosas estão associadas com o core Mon R2 $\left(L_{I R} \sim 26000 L_{\odot}\right)$ e o aglomerado jovem embebido GGD 12-15 $\left(L_{I R} \sim 5700 L_{\odot}\right)$.

A Tabela 3.4 lista as coordenadas centrais das sub-regiões selecionadas em Mon R2. Como foi salientado na Seção 3.3, essas áreas foram escolhidas para serem estudadas em 
Tabela 3.3 - Fontes pontuais IRAS associadas com a nuvem molecular Monoceros R2. Tabela baseada em Xie (1992).

\begin{tabular}{|c|c|c|c|c|c|c|c|}
\hline \multirow[b]{2}{*}{$\begin{array}{c}\text { IRAS PSC } \\
-\end{array}$} & \multirow[b]{2}{*}{$\begin{array}{l}\text { A.R. (J2000) } \\
\qquad(\mathrm{h} \mathrm{m} \mathrm{s})\end{array}$} & \multirow[b]{2}{*}{$\begin{array}{c}\operatorname{Dec}(\mathrm{J} 2000) \\
\left({ }^{\circ}, "\right)\end{array}$} & \multicolumn{4}{|c|}{$S_{\nu}$} & \multirow[b]{2}{*}{$\begin{array}{l}L_{I R} \\
\left(L_{\odot}\right)\end{array}$} \\
\hline & & & $\begin{array}{c}12 \mu \mathrm{m} \\
(\mathrm{Jy})\end{array}$ & $\begin{array}{c}25 \mu \mathrm{m} \\
(\mathrm{Jy})\end{array}$ & $\begin{array}{c}60 \mu \mathrm{m} \\
(\mathrm{Jy})\end{array}$ & $\begin{array}{c}100 \mu \mathrm{m} \\
(\mathrm{Jy})\end{array}$ & \\
\hline 06027-0714 & $06: 05: 08.2$ & $-07: 14: 42$ & $<0.25$ & 0.96 & 4.93 & 6.35 & $>7.7$ \\
\hline 06045-0554 & $06: 06: 58.5$ & $-05: 55: 08$ & 0.76 & 0.99 & 0.98 & 4.96 & 7.1 \\
\hline 06046-0536 & 06:07:06.7 & $-05: 37: 24$ & $<0.25$ & 0.25 & 3.42 & $<9.49$ & $>3.2$ \\
\hline 06046-0603 & $06: 07: 08.5$ & $-06: 03: 47$ & $<0.25$ & 2.24 & 15.3 & 28.3 & $>25.6$ \\
\hline 06047-0546 & $06: 07: 11.1$ & $-05: 47: 21$ & $<0.25$ & 1.66 & 4.83 & 3.96 & $>8.0$ \\
\hline 06049-0541 & $06: 07: 21.4$ & $-05: 41: 38$ & 0.36 & 1.84 & 4.47 & $<9.49$ & $>8.2$ \\
\hline 06049-0504 & $06: 07: 23.4$ & $-05: 04: 54$ & $<0.29$ & 0.36 & 1.16 & $<10.3$ & $>1.5$ \\
\hline 06050-0623 & $06: 07: 27.5$ & $-06: 23: 47$ & 11.8 & 119 & $<442$ & $<20190$ & $>240$ \\
\hline 06050-0509 & $06: 07: 31.6$ & $-05: 10: 21$ & $<0.39$ & 1.48 & 4.18 & 10.3 & $>9.2$ \\
\hline 06051-0653 & $06: 07: 33.5$ & $-06: 54: 26$ & $<0.25$ & 0.25 & 2.18 & $<5.21$ & $>2.2$ \\
\hline 06052-0533 & $06: 07: 43.5$ & $-05: 34: 17$ & $<0.25$ & 0.60 & 13.1 & 22.3 & $>19.1$ \\
\hline 06052-0512 & $06: 07: 45.0$ & $-05: 12: 40$ & $<0.25$ & 0.68 & 2.50 & $<15.9$ & $>3.2$ \\
\hline 06053-0622 & 06:07:46.7 & $-06: 23: 00$ & 470 & 4095 & 13070 & 20190 & 26008 \\
\hline 06053-0614 & $06: 07: 48.9$ & $-06: 14: 44$ & 0.78 & 1.07 & $<6.05$ & $<81.8$ & $>4.9$ \\
\hline 06054-0515 & $06: 07: 52.7$ & $-05: 16: 04$ & 0.84 & 1.37 & $<2.41$ & $<34.0$ & $>5.7$ \\
\hline 06055-0524 & 06:07:58.9 & $-05: 25: 03$ & $<0.45$ & 0.44 & 4.80 & $<10.4$ & $>4.7$ \\
\hline $06055-0653$ & $06: 07: 58.0$ & $-06: 53: 45$ & 0.52 & 2.11 & 3.85 & 4.76 & 10.3 \\
\hline 06056-0621 & $06: 08: 03.8$ & $-06: 21: 38$ & 7.07 & 29.3 & $<13070$ & $<20190$ & $>76.4$ \\
\hline 06056-0700 & $06: 08: 04.2$ & $-07: 00: 38$ & $<0.26$ & 0.69 & 2.24 & $<11.2$ & $>3.0$ \\
\hline 06058-0615 & $06: 08: 14.8$ & $-06: 15: 33$ & 0.71 & 0.83 & 18.4 & $<155$ & $>19.5$ \\
\hline 06059-0632 & $06: 08: 23.6$ & $-06: 33: 02$ & 0.38 & 4.42 & 9.64 & 6.52 & 18.9 \\
\hline 06060-0657 & $06: 08: 27.7$ & $-06: 57: 42$ & 0.92 & 1.57 & $<3.59$ & $<9.74$ & $>6.3$ \\
\hline 06060-0617 & $06: 08: 29.5$ & $-06: 18: 26$ & 0.28 & 1.23 & $<18.4$ & 39.0 & $>15.9$ \\
\hline 06068-0643 & $06: 09: 14.5$ & $-06: 43: 57$ & 0.73 & 1.35 & 1.91 & $<8.47$ & $>6.8$ \\
\hline 06068-0641 & $06: 09: 19.8$ & $-06: 41: 55$ & 0.98 & 2.00 & $<1.91$ & $<8.47$ & $>7.2$ \\
\hline 06070-0619 & $06: 09: 30.0$ & $-06: 19: 40$ & 2.95 & 15.2 & 178 & 314 & 287 \\
\hline 06084-0611 & $06: 10: 51.0$ & $-06: 11: 54$ & 27.1 & 604 & 3613 & 4876 & 5682 \\
\hline 06085-0613 & $06: 10: 57.8$ & $-06: 14: 37$ & 2.99 & 3.27 & $<3613$ & $<4876$ & $>17.5$ \\
\hline 06086-0611 & $06: 11: 07.5$ & $-06: 12: 32$ & 2.38 & 2.57 & $<3613$ & $<4876$ & $>13.9$ \\
\hline 06093-0608 & $06: 11: 48.6$ & $-06: 09: 30$ & 6.60 & 12.3 & 141 & 249 & 245 \\
\hline 06094-0628 & $06: 11: 53.3$ & $-06: 29: 20$ & $<0.25$ & 0.32 & 7.03 & 25.2 & $>14.6$ \\
\hline 06103-0612 & $06: 12: 48.3$ & $-06: 13: 19$ & 4.02 & 20.8 & 70.3 & 123 & 149 \\
\hline 06111-0624 & $06: 13: 36.2$ & $-06: 25: 01$ & 0.44 & 0.48 & 0.69 & $<11.2$ & $>3.1$ \\
\hline 06123-0619 & $06: 14: 44.9$ & $-06: 20: 24$ & 0.36 & 0.42 & $<1.87$ & 21.2 & $>9.1$ \\
\hline 06124-0621 & $06: 14: 53.1$ & $-06: 22: 43$ & 1.82 & 2.07 & 15.6 & 53.8 & 41.4 \\
\hline 06125-0658 & $06: 15: 00.8$ & $-06: 59: 15$ & $<0.25$ & 0.19 & $<0.51$ & 4.12 & $>1.6$ \\
\hline
\end{tabular}




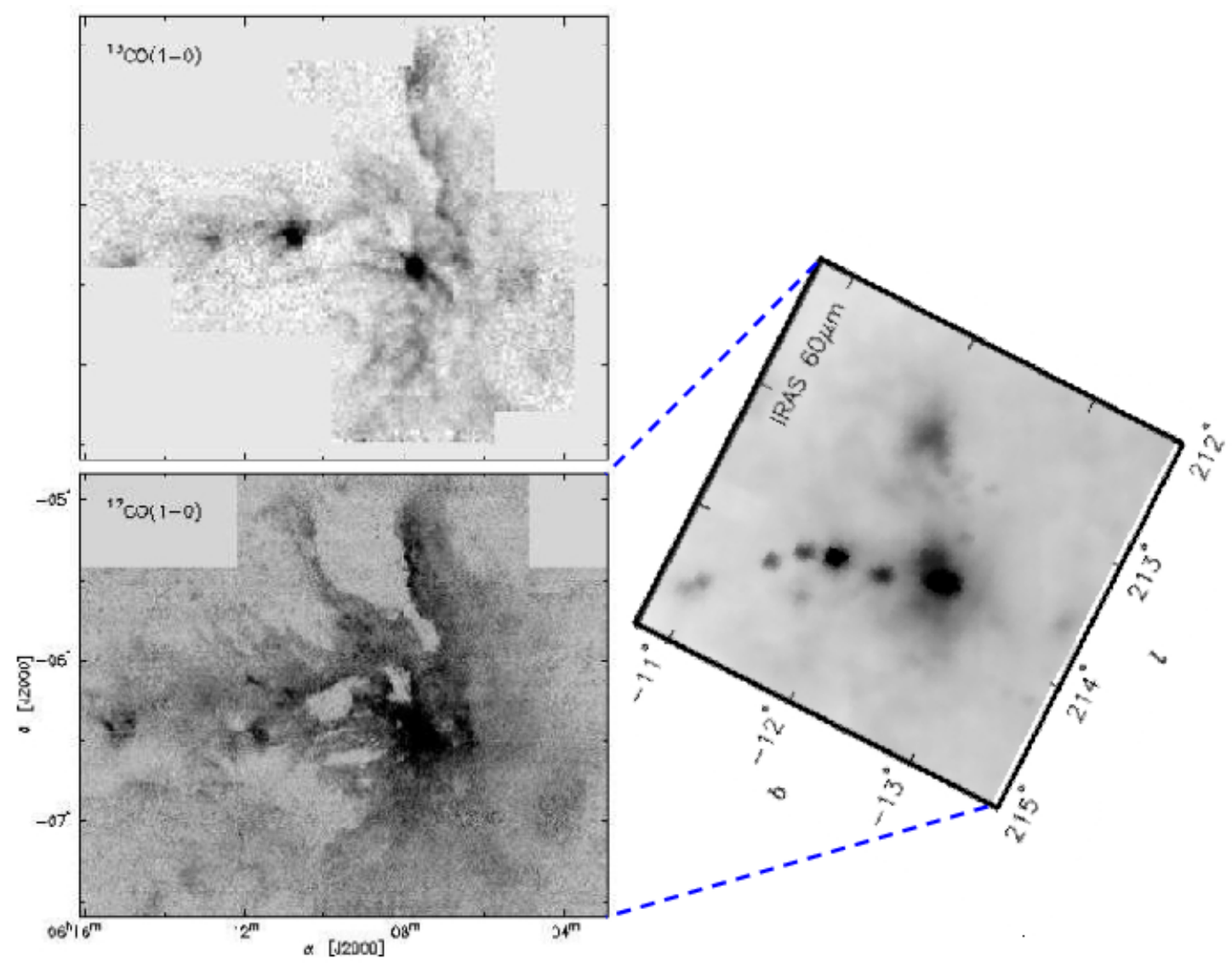

Figura 3.6: À esquerda são mostradas as distribuições de gás molecular em Mon R2, apresentando um mapa do pico de temperatura de antena medido para ${ }^{12} \mathrm{CO}$ (Xie, 1992), no painel inferior, e intensidade da emissão ${ }^{13} \mathrm{CO}$ (Miesch et al., 1999), no painel superior. O painel à direita apresenta um mapa (em coordenadas galácticas) da emissão em $60 \mu$ m medida com o IRAS, inclinado adequadamente para mostrar (de forma aproximada) sua relação com o mapa de $\mathrm{CO}$ (coordenadas equatoriais).

detalhe, pela coincidência espacial com regiões com maior concentração de indícios de formação estelar:

- Core de Mon R2: região central da nuvem Monoceros R2, contendo um grande número de objetos embebidos;

- GGD 12-15: algomerado estelar que possui associação com região HII compacta;

- GGD 16-17: região que abriga objetos jovens embebidos e interage com a estrela Bretz 4, uma T Tauri clássica (Carballo e Eiroa, 1992);

- HH866: objeto Herbig-Haro que está associado com a fonte IRAS 06046-0603, em região de baixa densidade estelar. 
Os quatro aglomerados possuem propriedades avaliadas por Carpenter (2000) e detalhadas na Tabela 3.4.

Finalmente, para completar nossa base de dados para Mon R2, a Tabela 3.5 traz as características dos masers de água detectados na região de nosso interesse, associados a fontes IRAS, os quais foram compilados por Codella et al. (1995) e Sunada et al. (2007), da mesma forma que em RMC. Assim como foi ressaltado para a Tabela 2.7, o trabalho de Codella et al. (1995) detecta apenas limites superiores nas medidas de fluxo da amostra. 
Tabela 3.4 - Características dos aglomerados estelares embebidos, presentes na nuvem Mon R2: coordenadas, número de membros e raio efetivo fornecidos por Carpenter (2000); extinção visual calculada por meio do programa KVIS.

\begin{tabular}{|c|c|c|c|c|c|c|c|c|c|}
\hline \multirow{3}{*}{ Região } & \multicolumn{4}{|c|}{ Coordenadas Centrais } & \multirow{3}{*}{$\mathrm{N}^{\circ}$ de estrelas } & \multirow{3}{*}{$\begin{array}{c}\text { Raio Efetivo } \\
\text { (pc) }\end{array}$} & \multicolumn{3}{|c|}{ Extinção Visual } \\
\hline & \multicolumn{2}{|c|}{ Galácticas } & \multicolumn{2}{|c|}{ Equatoriais (J2000) } & & & Mín & Máx & Méd \\
\hline & $1\left(^{\circ}\right)$ & $\mathrm{b}\left({ }^{\circ}\right)$ & A.R. (h m s) & $\operatorname{Dec}\left({ }^{\circ}, "\right)$ & & & (mag) & (mag) & (mag) \\
\hline Mon R2 & 213.69 & -12.59 & $06: 07: 47.8$ & $-06: 22: 20$ & 371 & 1.85 & 1.31 & 2.69 & 1.91 \\
\hline GGD $12-15$ & 213.87 & -11.84 & $06: 10: 49.1$ & $-06: 11: 38$ & 134 & 1.13 & 0.83 & 1.99 & 1.59 \\
\hline GGD $16-17$ & 214.13 & -11.42 & $06: 12: 48.0$ & $-06: 13: 56$ & 23 & 0.61 & 0.93 & 2.03 & 1.56 \\
\hline HH866 & 213.34 & -12.60 & $06: 07: 08.1$ & $-06: 03: 53$ & 15 & 0.41 & 1.78 & 2.32 & 2.08 \\
\hline
\end{tabular}

Tabela 3.5 - Masers de água detectados em Mon R2.

\begin{tabular}{cccccccc}
\hline \hline IRAS PSC & $\mathrm{l}$ & $\mathrm{b}$ & $\begin{array}{c}\text { A.R. (J2000) } \\
(\mathrm{h} \mathrm{m} \mathrm{s})\end{array}$ & $\begin{array}{c}\text { Dec }(\mathrm{J} 2000) \\
\left({ }^{\circ},{ }^{\prime}\right)\end{array}$ & $\begin{array}{c}\mathrm{A}_{V} \\
(\mathrm{mag})\end{array}$ & $\begin{array}{c}\mathrm{F}_{\text {pico }} \\
(\mathrm{Jy})\end{array}$ & Referência \\
\hline 06343-1036 & 220.81 & -08.07 & $06: 36: 43.1$ & $-10: 38: 44$ & 1.14 & $<3.0$ & Codella et al. (1995) \\
$06057-0923$ & 216.52 & -13.86 & $06: 08: 05.1$ & $-09: 23: 52$ & 0.67 & $<5.7$ & Codella et al. (1995) \\
$06010-0943$ & 216.32 & -15.03 & $06: 03: 28.3$ & $-09: 43: 40$ & 0.8 & $<5.4$ & Codella et al. (1995) \\
$05598-0906$ & 215.61 & -15.03 & $06: 02: 16.3$ & $-09: 06: 32$ & 0.43 & $<5.7$ & Codella et al. (1995) \\
$06056-0700$ & 214.31 & -12.82 & $06: 08: 04.2$ & $-07: 00: 38$ & 1.73 & $<5.4$ & Codella et al. (1995) \\
$06103-0612$ & 214.12 & -11.41 & $06: 12: 50.4$ & $-06: 13: 11$ & 1.25 & $<6.0$ & Codella et al. (1995) \\
$06070-0619$ & 213.85 & -12.19 & $06: 09: 30.0$ & $-06: 19: 40$ & 1.06 & $<4.5$ & Codella et al. (1995) \\
$06060-0617$ & 213.71 & -12.41 & $06: 08: 29.5$ & $-06: 18: 26$ & 1.5 & $<6.6$ & Codella et al. (1995) \\
$06046-0603$ & 213.34 & -12.60 & $06: 07: 08.4$ & $-06: 03: 47$ & 1.83 & $<6.3$ & Codella et al. (1995) \\
$06050-0509$ & 212.57 & -12.12 & $06: 07: 31.6$ & $-05: 10: 20$ & 1.26 & $<4.8$ & Codella et al. (1995) \\
$06052-0533$ & 212.95 & -12.25 & $06: 07: 43.5$ & $-05: 34: 17$ & 1.24 & 1.09 & Sunada et al. (2007) \\
$06053-0622$ & 213.70 & -12.60 & $06: 07: 46.7$ & $-06: 23: 00$ & 1.86 & 100.56 & Sunada et al. (2007) \\
$06084-0611$ & 213.88 & -11.83 & $06: 10: 51.5$ & $-06: 11: 27$ & 1.4 & 9.04 & Sunada et al. (2007) \\
$06103-0612$ & 214.13 & -11.41 & $06: 12: 48.3$ & $-06: 13: 19$ & 1.25 & 46.07 & Sunada et al. (2007) \\
\hline
\end{tabular}


Capítulo 4

\section{Metodologia e Análise Comparativa}

O objetivo do presente capítulo é buscar por correlações entre as características levantadas nos Capítulos 2 e 3 para as regiões estudadas por nós. Descrevemos inicialmente a metodologia adotada, que envolve a construção de mapas nos quais se combinam diferentes bandas espectrais. Em seguida, a distribuição espacial de alguns objetos selecionados é confrontada com os mapas, buscando verificar diferenças e semelhanças na associação nuvem-objeto jovem.

O levantamento de objetos associados à nuvem, cujas propriedades são consistentes com regiões de formação estelar, nos permite explorar sua natureza e conhecer melhor o cenário de evolução de protoestrelas presentes em cada uma das nuvens, primeiramente em uma escala global, para posteriormente nos aprofundarmos em uma escala mais local.

O esquema mostrado na Figura 4.1, que se trata de uma sobreposição das Figuras 2.1 e 3.1, ilustra em grande escala como se distribuem espacialmente as principais regiões estudadas por nós. Todos os mapas produzidos no presente trabalho, visando comparar as características estimadas em diferentes comprimentos de onda, referem-se ao zoom que se aplica para as regiões e objetos de interesse, como descrevemos a seguir.

No contexto em grande escala do Complexo de Monoceros, nossa coleta de informações se restringiu à região denominada OB2, $\left(\delta_{1950}<8^{\circ}\right)$. Mais ao norte, nas proximidades do plano Galáctico, encontra-se RMC abrangendo uma região de $3^{\circ} \times 3^{\circ}$, que pode ser tratada em "grande escala" com relação às demais. Entre várias sub-regiões de interesse em RMC destacam-se a Nebulosa Rosette (centrada no aglomerado NGC2244), além de outros grupos estelares identificados no infravermelho, bem como muitos starless cores, ou clumps, associados a estrelas massivas, os quais são tratados em "pequena escala" (5' x 5') 


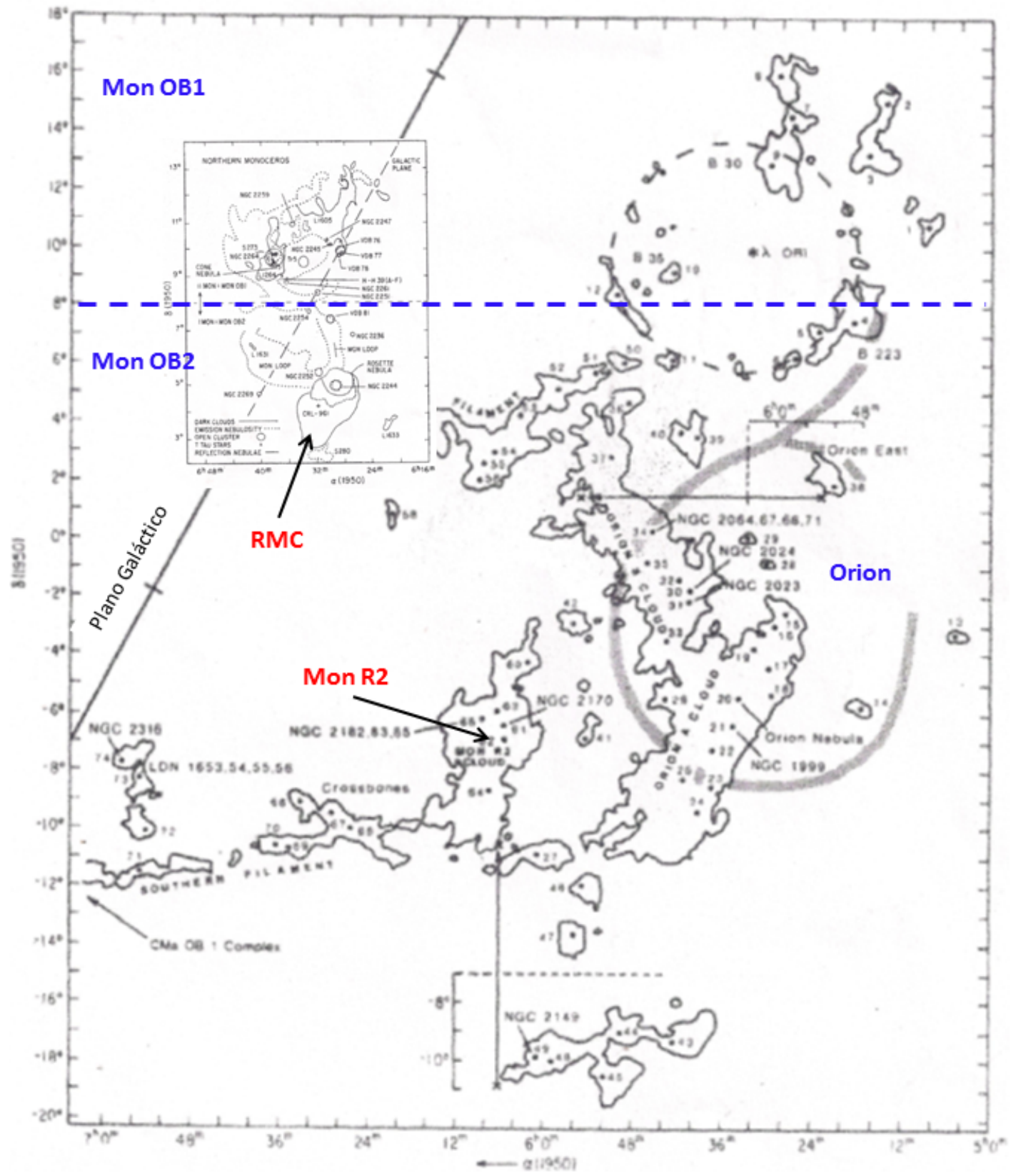

Figura 4.1: Sobreposição dos esquemas apresentados individualmente nas Figuras 2.1 e 3.1, para ilustrar e comparar a distribuição espacial, em grande escala, das nuvens RMC e Mon $\mathrm{R} 2$.

em nosso estudo comparativo.

A nuvem localizada mais ao sul, a cerca de $12^{\circ}$ do plano Galático, contém várias nebulosas de reflexão e denomina-se Mon R2, contendo um core denso principal, e pelo menos três outros objetos interessantes a serem estudados em menor escala.

A Tabela 4.1 estabelece a denominação adotada para cada região ou sub-região e separa 
as diferentes escalas a na nossa metodologia de estudo. Além disso, são informados os surveys e catálogos utilizados para diferentes fins específicos, em termos de traçadores da formação estelar e/ou deteç̧ão da presença de estrelas jovens.

Tabela 4.1 - Descrição dos mapas construídos no presente trabalho, em função das diferentes escalas e características observadas a partir dos surveys e catálogos adotados.

\begin{tabular}{|c|c|c|c|c|}
\hline \multicolumn{2}{|c|}{ Região } & Escala & Catálogo & Mapeamento \\
\hline \multicolumn{2}{|c|}{ Complexo Mon OB2 } & $25^{\circ} \times 25^{\circ}$ & $\mathrm{A}_{V}$ e CO & Nuvens escuras \\
\hline RMC & Mon R2 & $3^{\circ} \times 3^{\circ}$ & CO e IRAS & Gás e poeira \\
\hline Nebulosa Rosette & Mon R2 central & $1^{\circ} \times 1^{\circ}$ & IRAS e DSS & Nebulosidades \\
\hline Clusters A, B, C e D & $\begin{array}{c}\text { Mon R2 core, GGD 12-15, } \\
\text { GGD 16-17, HH866 }\end{array}$ & $5^{\prime} \times 5^{\prime}$ & $2 M A S S$ & $\begin{array}{c}\text { Aglomerados } \\
\text { estelares }\end{array}$ \\
\hline
\end{tabular}

Após a construção dos mapas, em diferentes bandas espectrais e diferentes escalas, foi avaliada a distribuição espacial dos objetos estelares selecionados (listados nas tabelas apresentadas nos Capítulos 2 e 3). Os processos energéticos, indicados pela presença de estrelas massivas detectadas em raios-X, configuram-se como ponto de partida na busca de alvos a serem selecionados para o estudo das nuvens no Complexo Monoceros. Resultados das informações combinadas entre raios-X, óptico, infravermelho e rádio podem contribuir para avanços no conhecimento de objetos pré-estelares e suas condições de formação e evolução.

\subsection{Distribuição das nuvens: mapas de extinção, gás molecular e poeira}

Apresentamos aqui os mapas construídos em maior escala, combinando dados ópticos, que revelam a extinção causada pelas nuvens escuras, com os dados da emissão CO e a distribuição da poeira fria, revelada pela emissão em $100 \mu \mathrm{m}$, medida pelo IRAS.

A Figura 4.2 ilustra a posição das nuvens RMC e Mon R2 no contexto do Complexo de Monoceros e mostra a posição de CMa R1, M 42 (em Orion) e NGC2264 (em RMC) para referência na localização espacial. Essa combinação de informações nos fornece indícios sobre a relação entre a distribuição da poeira (inferida pela magnitude da extinção visual - $\mathrm{A}_{V}$ ) e do gás (pela distribuição da emissão de $\mathrm{CO}$ ). O mapa de $\mathrm{A}_{V}$ foi obtido de Dobashi et al. (2005) e o mapa de CO foi fornecido por Dame et al. (2001).

Em um contexto mais específico, as Figuras 4.3 e 4.4 mostram para RMC e Mon R2, respectivamente, a distribuição de gás em tons de cinza, correlacionada com a emissão 


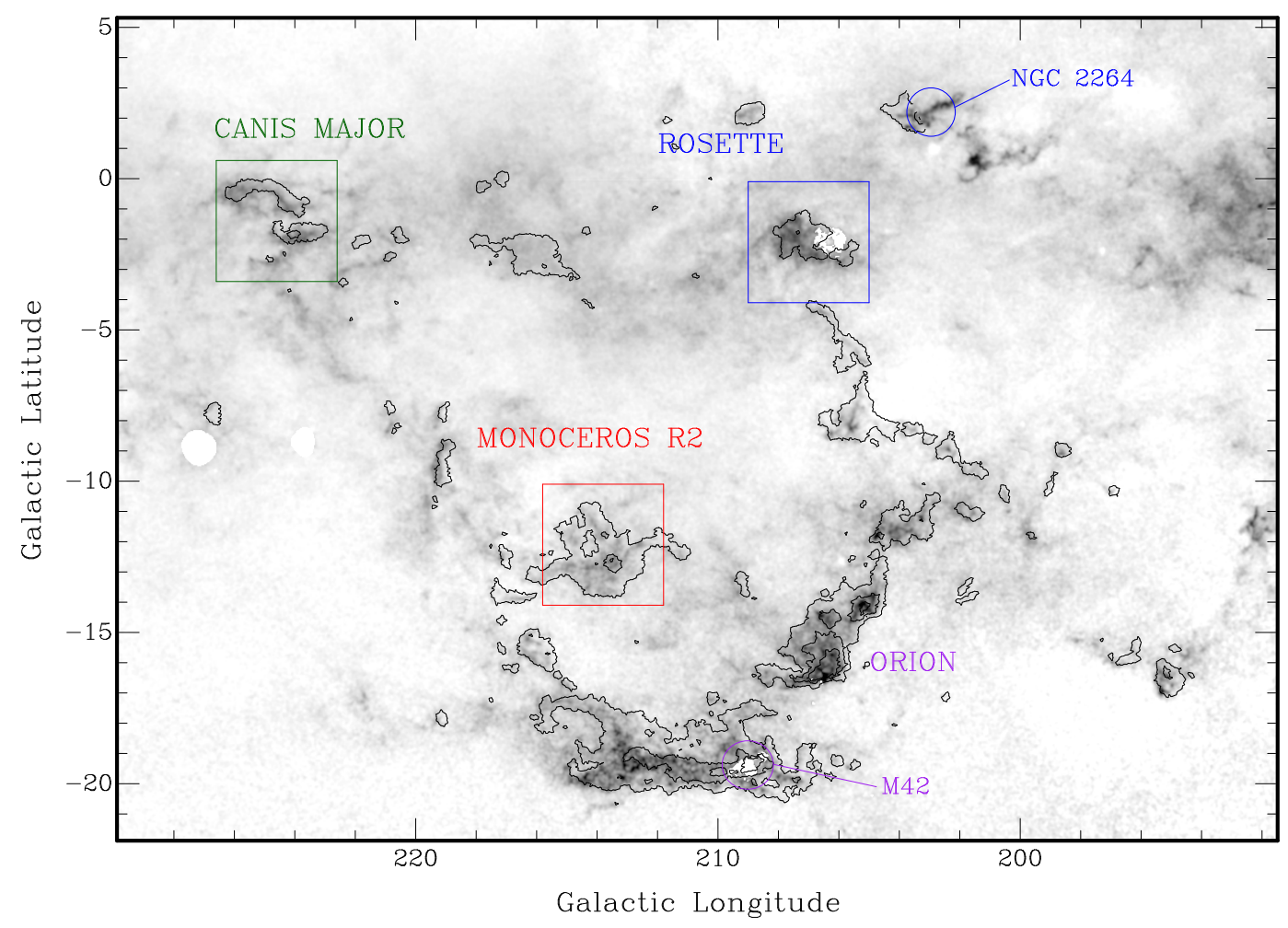

Figura 4.2: Complexo de Monoceros: mapa espacial de nuvens moleculares, exibindo contornos de CO (Dame et al., 2001), em 10 e $50 \mathrm{Kkm}^{-1}$, sobre mapa de $\mathrm{A}_{V}$ (Dobashi et al., 2005). Em tons de cinza, regiões mais escuras representam altos níveis de extinção $\left(\mathrm{A}_{V}>\right.$ $3.0 \mathrm{mag}$ ). Os píxeis em branco correspondem a regiões de $A_{V}$ indeterminado (presença de estrelas brilhantes).

no infravermelho distante apresentada em contornos. Dessa forma, é possível conhecer a distribuição do gás em relação às regiões de alta concentração de poeira revelada pela emissão em $100 \mu \mathrm{m}$.

\subsection{Comparação do conteúdo estelar com as regiões densas}

Bloemen et al. (1990) comparam os dados CO com a emissão em 60 e $100 \mu \mathrm{m}$, medida pelo IRAS em toda a Galáxia, para separar as componentes associadas com o gás atômico e o molecular. Eles utilizam a cor dada pela razão de fluxos $\mathrm{F}_{60} / \mathrm{F}_{100}$ para identificar a emissão no infravermelho distante, que é dominada pela poeira morna associada a regiões 


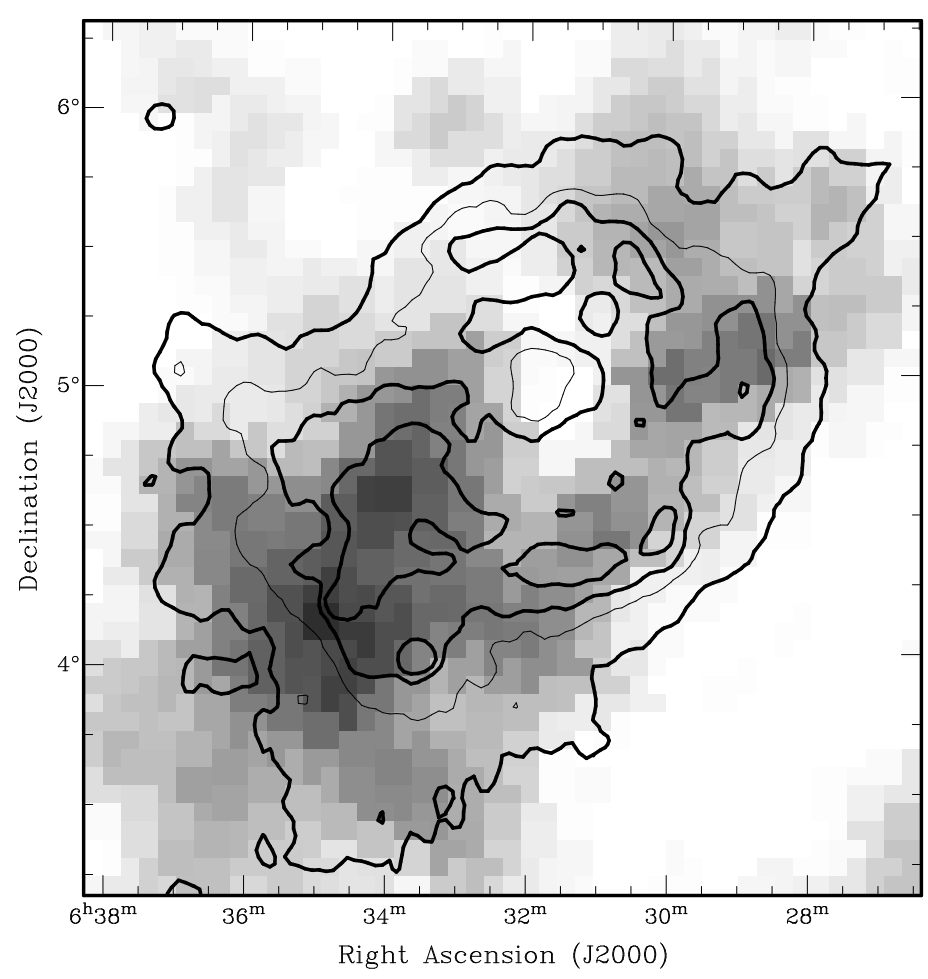

Figura 4.3: Nuvem molecular Rosette. Contornos de 50, 95, 150 e $250 \mathrm{MJy} / \mathrm{sr}$ em $100 \mu \mathrm{m}$ (IRAS) sobrepostos a imagem de CO de Dame et al. (2001). Em tons de cinza, regiões mais escuras do mapa representam alta densidade do gás.

HII. Com base na mesma idéia, de destacar a emissão infravermelha devida a presença do conteúdo estelar contrastando-a com a emissão fria devida aos cores densos sem a presença de estrelas, elaboramos um mapa que corresponde à razão entre fluxos $\mathrm{F}_{60} / \mathrm{F}_{100}$. Para isso, a imagem obtida pelo IRAS na banda de $100 \mu \mathrm{m}$ foi dividida pela equivalente (mesma região) em $60 \mu \mathrm{m}$.

A Figura 4.5 mostra o mapa de extinção visual sobreposto pelos contornos da razão de fluxos $\mathrm{F}_{60} / \mathrm{F}_{100}$, obtido em maior escala, para o Complexo de Monoceros. Nota-se uma boa correlação com a distribuição das nuvens escuras, porém em algumas áreas de maior concentração de poeira (maior $A_{V}$ ) a razão $\mathrm{F}_{60} / \mathrm{F}_{100}$, aparece menos intensa, indicando a ausência de emissão estelar nesses casos.

Os contornos da emissão em $100 \mu$ m também foram sobrepostos à imagem óptica obtida pelo DSS (Digitized Sky Survey), na banda azul $(\lambda=4500-4950 \AA)$. Dessa forma, é possível comparar na Figura 4.6 a posição das estrelas e a região de concentração de poeira que revela altas densidades da nuvem em RMC. Por meio da Figura 4.7, que mostra a imagem 


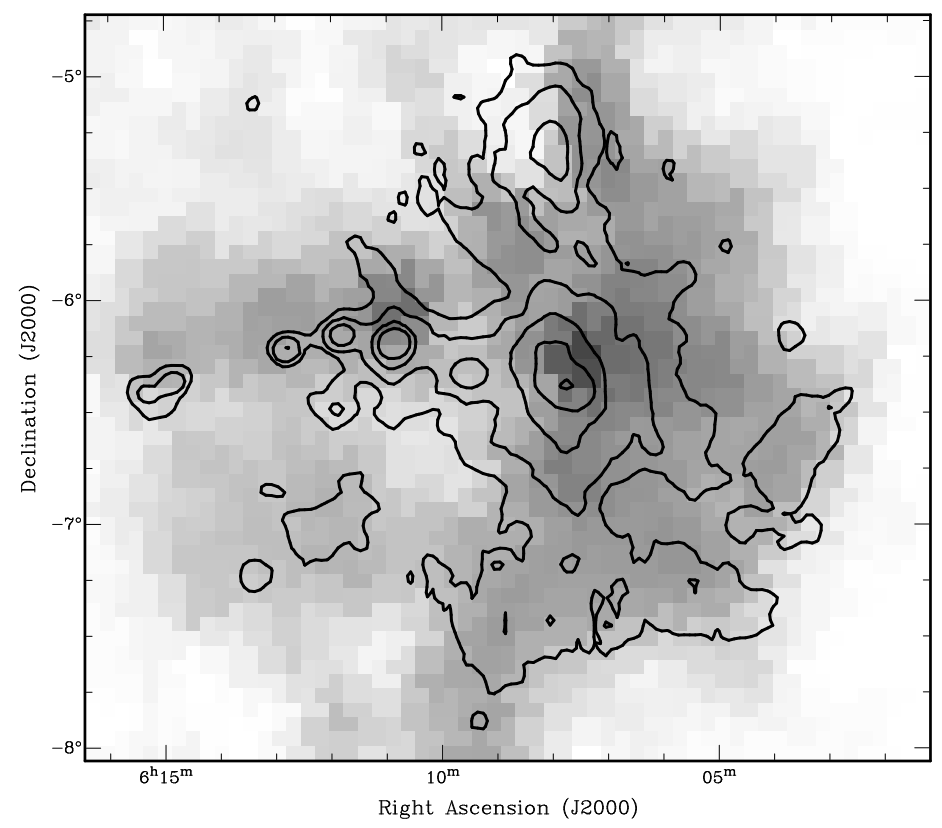

Figura 4.4: Nuvem Mon R2. Idem à Fig. 4.3, exceto pelos níveis de contornos IRAS que neste caso correspondem a 20,60, 40 e $80 \mathrm{MJy} / \mathrm{sr}$.

$D S S$ para Mon R2, podemos verificar a localização das sub-regiões de interesse, que foram selecionadas de acordo com a descrição a seguir. 


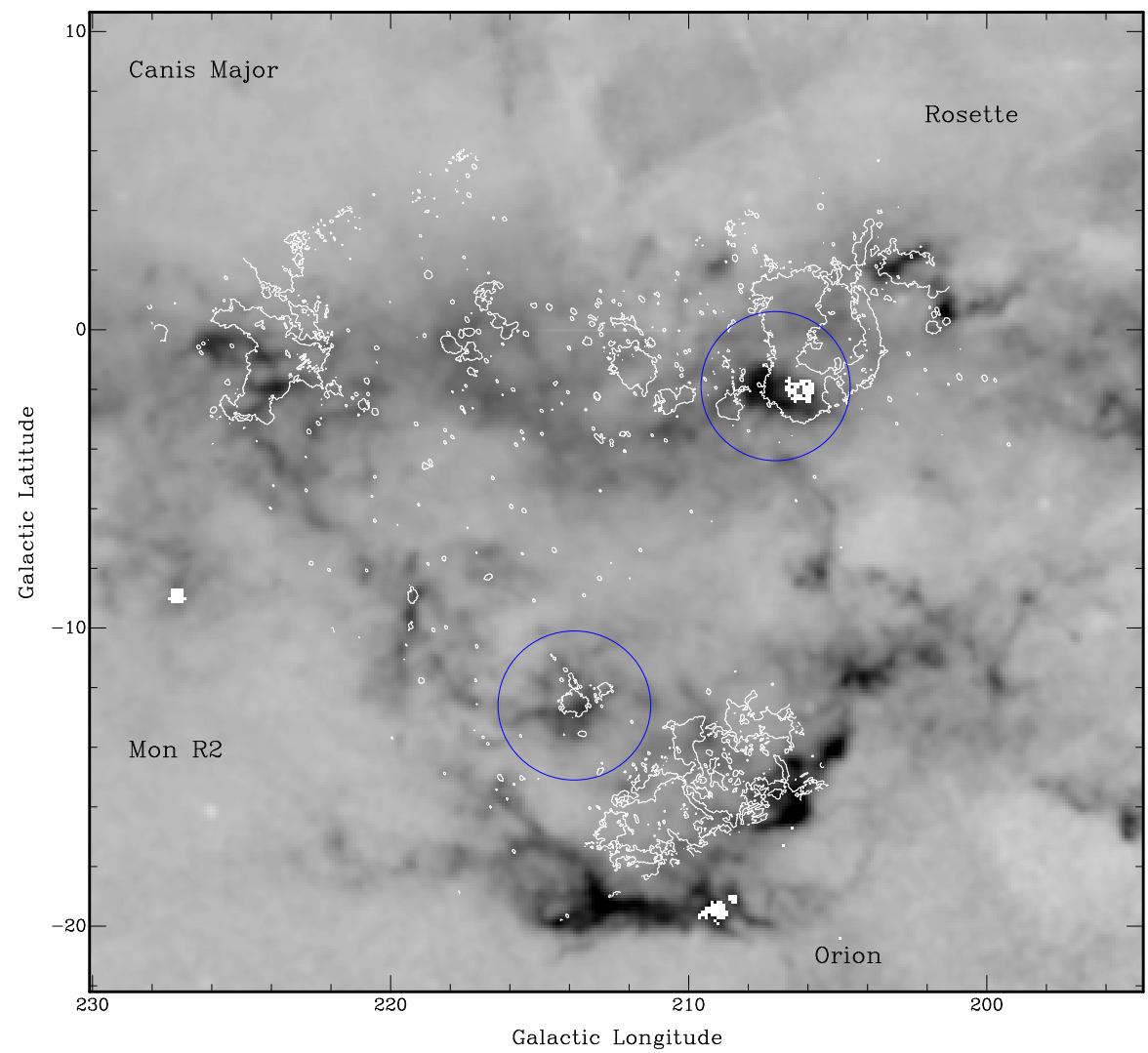

Figura 4.5: Mapa de extinção visual sobreposto pelo resultado da razão $\mathrm{F}_{60} / \mathrm{F}_{100}$ obtida a partir das imagens IRAS. O nível do contorno indicado é de 0.27 , valor adotado por Bloemen et al. (1990) para regiões galácticas de poeira misturada com gás.

\subsection{Seleção das sub-regiões de interesse}

A metodologia para a identificação dos candidatos a objetos pré-estelares associados a RMC consistiu em uma inspeção visual em imagens ( $D S S$ ) buscando os objetos nas proximidades das estrelas massivas presentes na região. A partir das características de proplyds (objetos com disco protoplanetário) detectados em Orion (Shuping et al., 2006; De Marco et al., 2006), foi realizado um estudo comparativo buscando estimar a dimensão esperada para os clumps localizados em RMC, que se encontra a cerca de 1600 pc. Adotando-se uma dimensão típica de 500 UA para a estrutura circunstelar, o tamanho angular dos clumps seria de aproximadamente 0.3 arcmin.

Como resultado, foram selecionados cerca de 70 candidatos a objetos pré-estelares em 


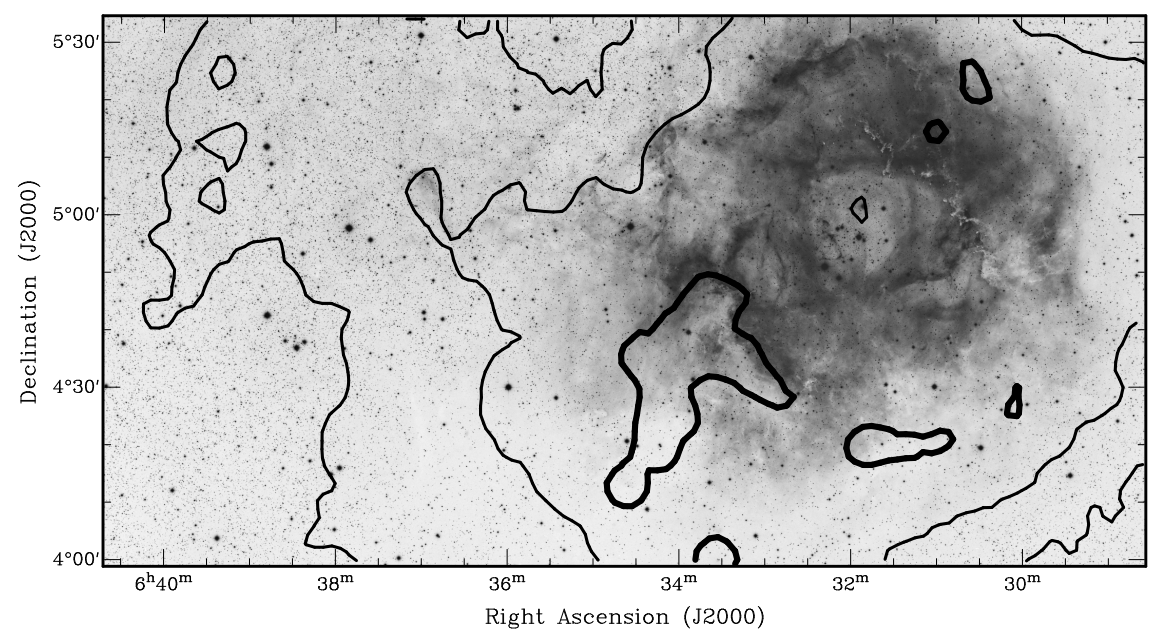

Figura 4.6: Contornos de 20, 30, 75, e $300 \mathrm{MJy} / \mathrm{sr}$ em $100 \mu \mathrm{m}$ (IRAS) sobre imagem óptica $(D S S)$ na banda azul, cobrindo a nuvem molecular Rosette. Em tons de cinza, regiões mais escuras correspondem a fluxos mais altos.

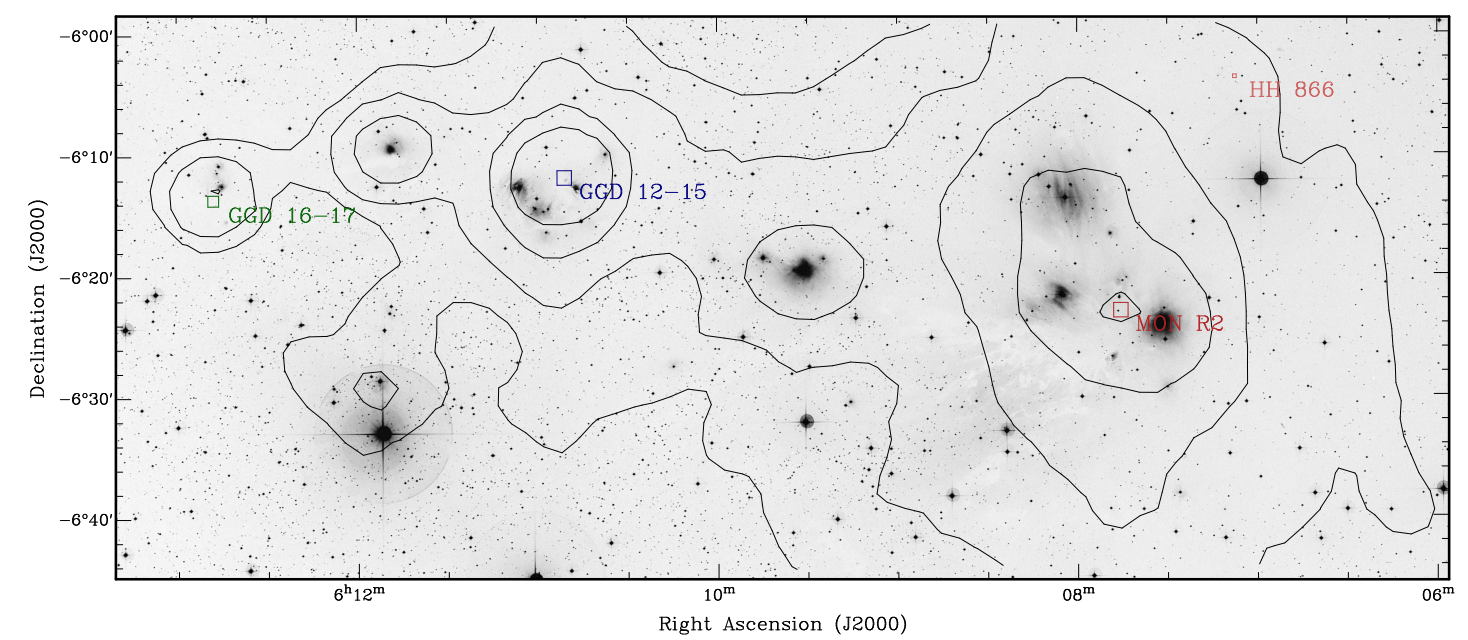

Figura 4.7: Contornos de 20,30, 75 e $300 \mathrm{MJy} / \mathrm{sr}$ em $100 \mu \mathrm{m}$ (IRAS) sobre imagem óptica $(D S S)$ na banda azul, cobrindo a nuvem Monoceros R2. Em tons de cinza, regiões mais escuras correspondem a fluxos mais altos. Destaque para as sub-regiões: core Mon R2, GGD 12-15, GGD 16-17 e HH866. 
RMC (Williams et al., 1995), para os quais foram analisadas as imagens no óptico e no infravermelho distante. Gregorio-Hetem et al. (1998) apresentam 21 fontes de raios-X detectados pelo ROSAT, das quais 14 fontes possuem contrapartida óptica. O satélite Chandra permitiu maior sensibilidade de detecção de fontes-X, como mostra o trabalho de Wang et al. (2008), que infere a presença de mais de 1700 YSOs em RMC. A posição dos clumps foi comparada com a posição de fontes pontuais IRAS associadas a RMC e encontramos clumps aparentemente associados a fontes ROSAT.

Como mencionado no Capítulo 3, a lista de objetos interessantes em Mon R2 foi baseada em três trabalhos anteriores. Carpenter (2000) utilizou observações 2MASS (infravermelho próximo), para identificar 4 aglomerados embebidos (totalizando 543 estrelas) e descrevêlos em termos de população estelar. Xie (1992) estudou objetos de baixa temperatura e pouca luminosidade, detectados pelo survey IRAS, e selecionou 36 fontes infravermelhas de acordo com os critérios descritos na Seção 3.4. A emissão de raios-X foi estudada por Gregorio-Hetem et al. (1998), que confirmaram 41 fontes-X detectadas pelo satélite ROSAT, e por Nakajima et al. (2003), que utilizaram dados Chandra para identificar 368 fontes, concluindo que as fontes X correlacionam-se com a formação estelar ativa no core da nuvem.

Desta forma, verifica-se que centenas de fontes pontuais identificadas pelo Chandra correspondem a apenas algumas poucas fontes detectadas pelo ROSAT como sendo extensas. Isso é devido às diferenças na resolução espacial e na sensibilidade dos dois surveys realizados em Mon R2. Considerando que não estamos interessados em um detalhamento individual das fontes com emissão-X, mas sim uma visão geral e em maior escala das regiões de interesse, optamos por adotar as coordenadas das fontes ROSAT como indicativos da posição dos aglomerados de fontes-X detectadas pelo Chandra, em vez de utilizar as coordenadas de todas fontes-X pontuais conhecidas na região, tanto para Mon R2 como para RMC.

Em complemento à lista de candidatas, incluimos as 7 fontes maser de $\mathrm{H}_{2} \mathrm{O}$ encontradas na região de RMC e 14 masers em Mon R2.

Para avaliar a distribuição de candidatos a objetos jovens na região, correlacionamos a posição dos objetos selecionados, sobrepondo-os inicialmente ao mapa IRAS da emissão em $100 \mu \mathrm{m}$, que tem uma boa correspondência com o mapa de extinção visual, bem como 
com a distribuição de gás molecular (vide Figuras 4.3 e 4.4).

Os resultados da comparação com os mapas IRAS são mostrados nas Figuras 4.8 e 4.9, respectivamente para RMC e Mon R2. Nem todas estrelas massivas da Tabela 2.1 foram inseridas no gráfico que corresponde à distribuição de objetos em RMC. Os motivos são: (i) a grande concentração de estrelas tipo O no centro da Nebulosa Rosette (NGC2244), não trazendo informações adicionais sobre sua distribuição e correlação com outros objetos, e (ii) não há em Mon R2 um número de estrelas massivas comparável àquelas associadas a NGC2244. Desta forma, optamos por utilizar na Figura 4.8 apenas as estrelas tipo B com $\mathrm{m}_{V}>10$ mag para ilustrar sua distribuição espacial, limitando a amostra de RMC com características semelhantes às de Mon R2. 


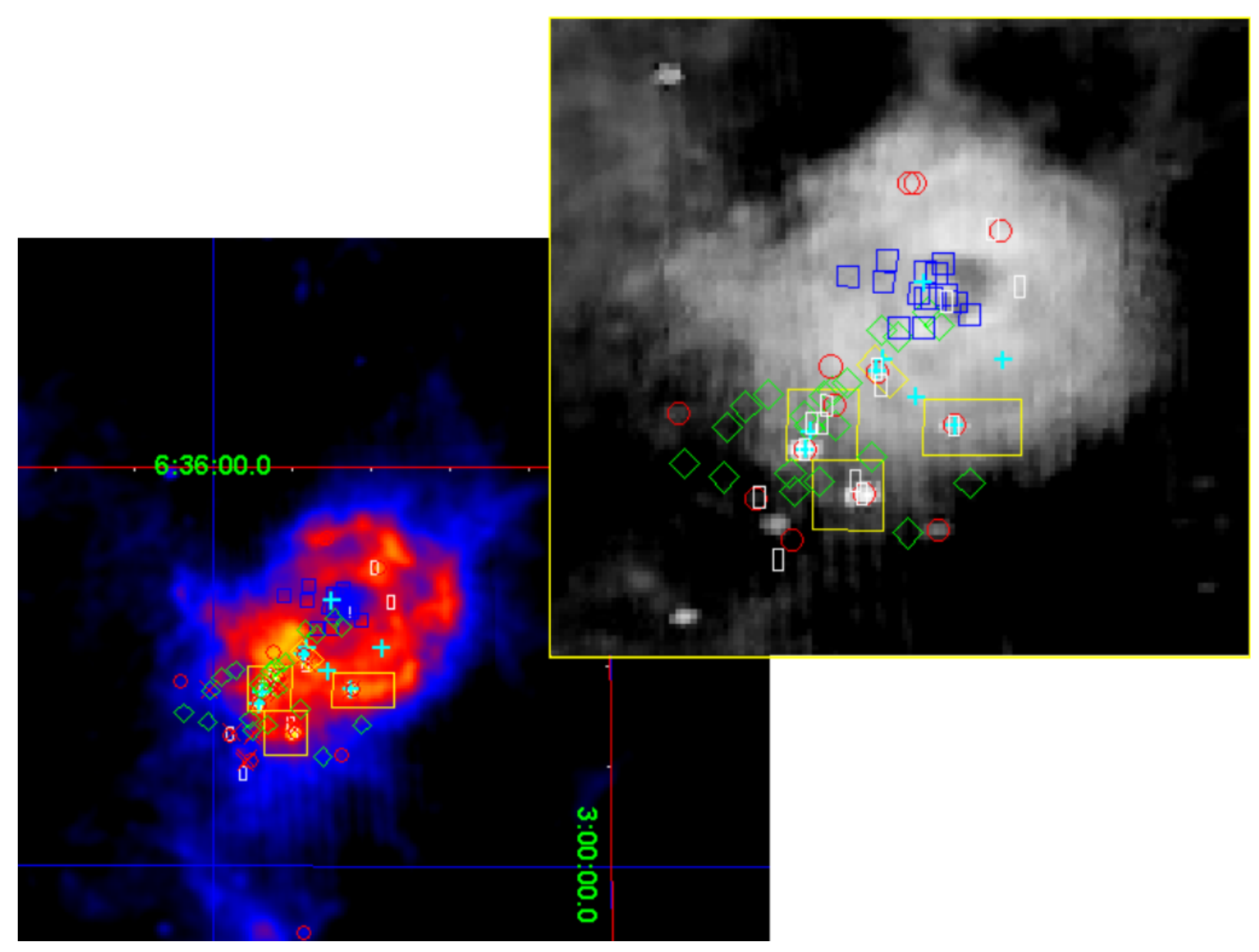

Figura 4.8: Distribuição de objetos associados a RMC. A imagem colorida mostra em maior escala o detalhamento da emissão em $100 \mu \mathrm{m}$ e a grade de coordenadas com separação de $3^{\circ}$ entre as linhas. A imagem em níveis de cinza mostra um zoom da região central, com maior detalhamento para a posição das fontes. Além das 4 sub-regiões principais de nosso interesse (retângulos amarelos), são indicadas algumas das estrelas tipo B (quadrados azuis), as fontes IRAS (círculos vermelhos); as fontes ROSAT (losângulos verdes), as fontes maser (cruzes), e os clusters de fontes 2MASS (retângulos brancos). Alguns dos clumps da Tabela 2.6, selecionados pela coincidência de posição com outros objetos de interesse, são indicados por "X" em vermelho, na imagem à esquerda.

Considerando que a distribuição espacial dos 70 clumps identificados em RMC por Williams et al. (1995) não apresentou correlação com emissão em $100 \mu \mathrm{m}$, decidimos não apresentar todos eles na Figura 4.8. Além disso, não há uma amostra similar identificada em Mon R2, impossibilitando a análise comparativa baseada nesse tipo de informação. Desta forma, optamos por incluir no gráfico da Figura 4.8 apenas alguns clumps coincidentes com a posição dos demais objetos selecionados na RMC. 


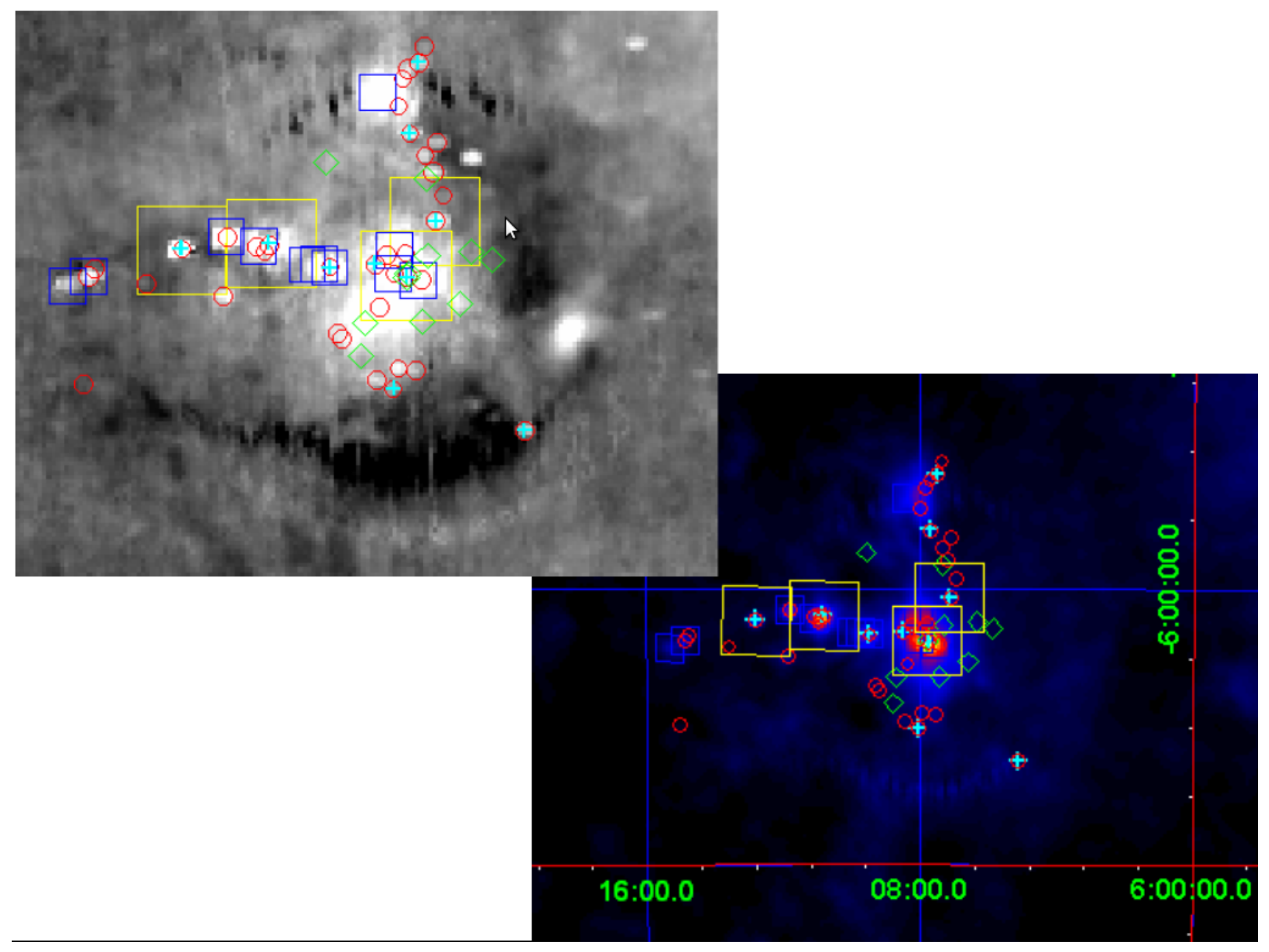

Figura 4.9: Distribuição de objetos associados a Mon R2, sobrepostos à imagem IRAS. Idem à Figura 4.8, porém com separação de $2^{\circ}$ entre as linhas da grade de coordenadas. Além das 4 sub-regiões principais de nosso interesse (quadrados amarelos), são indicadas as estrelas tipo B (quadrados azuis), as fontes IRAS (círculos vermelhos); as fontes ROSAT (losângulos verdes), e as fontes maser (cruzes).

A Tabela 4.2 traz a lista de objetos identificados em RMC, para os quais foi verificada coincidência na posição entre diferentes tipos de fontes. Notamos que a maior concentração desses objetos ocorre nas regiões A, B, C e D, definidas por Li e Smith (2005), com base nas maiores aglomerações de fontes $2 M A S S$ (vide Tabela 2.4). Mais especificamente, as 4 sub-regiões escolhidas para serem estudadas detalhadamente em RMC contém 9 dos aglomerados jovens listados na Tabela 2.2, identificados por: PL01 (sub-região A), PL02 e PouC (sub-região B), PL04, PL05, PL06, e REFL08 (sub-região C), e PL03 e PouD (sub-região D). 
Tabela 4.2 - Objetos selecionados em RMC, que se apresentam associadas a diferentes tipos de fontes.

\begin{tabular}{|c|c|c|c|c|c|c|}
\hline Região $^{1}$ & IRAS & Estrela $^{2}$ & $R X J^{3}$ & Maser ${ }^{4}$ & Clump ${ }^{5}$ & Cluster $^{6}$ \\
\hline $\mathrm{A}$ & $06291+0420$ & $\ldots$ & $\ldots$ & $206.84,-2.38$ & 11 & PL01 \\
\hline \multirow[t]{3}{*}{$\mathrm{B}$} & $06306+0437$ & $\ldots$ & $\ldots$ & $206.78,-1.94$ & 18 & PL02 \\
\hline & $06305+0440$ & $\operatorname{HD} 259532^{a}$ & $\ldots$ & $206.72,-1.94$ & $\ldots$ & $\ldots$ \\
\hline & $\ldots$ & $\ldots$ & $\operatorname{RMCX} 70^{(g)}$ & $\ldots$ & $\ldots$ & PouC \\
\hline \multirow[t]{5}{*}{$\mathrm{C}$} & $06314+0427$ & GSC 00154-00020 & $0634.1+0429$ & $\ldots$ & 01 & PL04 \\
\hline & $06318+0420$ & $\ldots$ & $0634.5+0419$ & $207.18,-1.79$ & 06 & PL05 \\
\hline & $06321+0424$ & $\ldots$ & $0634.6+0422$ & $\ldots$ & $\ldots$ & PL05 \\
\hline & $06314+0421$ & $\ldots$ & $0634.0+0419$ & $\ldots$ & 19 & REFLO \\
\hline & $06319+0415$ & $\ldots$ & $\ldots$ & $207.27,-1.81$ & 02 & PL06 \\
\hline \multirow[t]{2}{*}{$\mathrm{D}$} & $06308+0402$ & $\ldots$ & $\ldots$ & $\ldots$ & 07 & PL03/PouD \\
\hline & $\ldots$ & GCS 00154-00622 & $0634.3+0403$ & $\ldots$ & 16 & $\ldots$ \\
\hline \multirow[t]{9}{*}{ Outras } & $06329+0401$ & $\ldots$ & $\ldots$ & $\ldots$ & 03 & PL07 \\
\hline & $\ldots$ & $\ldots$ & $\ldots$ & $\ldots$ & 05 & REFL09 \\
\hline & $06298+0430$ & GSC $00154-00304^{b}$ & $\ldots$ & $206.8,-2.15$ & $\ldots$ & $\ldots$ \\
\hline & $06312+0434$ & $\mathrm{HD} 46485^{c}$ & $0633.8+0431$ & $\ldots$ & $\ldots$ & $\ldots$ \\
\hline & $\ldots$ & GSC 00154-01628 & $0633.6+0418$ & $\ldots$ & 12 & $\ldots$ \\
\hline & $06322+0349$ & $\ldots$ & $\ldots$ & $\ldots$ & 05 & $\ldots$ \\
\hline & $06282+0440$ & HD $35891^{d}$ & $\ldots$ & $206.46,-2.44$ & $\ldots$ & $\ldots$ \\
\hline & $\ldots$ & $\mathrm{HD} 46301^{e}$ & $0632.6+0349$ & $\ldots$ & $\ldots$ & $\ldots$ \\
\hline & $\ldots$ & HD $259512^{e}$ & $0633.2+0446$ & $\ldots$ & $\ldots$ & $\ldots$ \\
\hline
\end{tabular}

Identificações: (1) De acordo com as identificações de Li e Smith (2005); (2) Candidata a contrapartida óptica, indicando tipo espectral e magnitude visual, (quando disponível); (3) Fontes detectadas pelo ROSAT, publicadas por Gregorio-Hetem et al. (1998); (4) Posição do maser de $\mathrm{H}_{2} \mathrm{O}$ em coordenadas galácticas; (5) Identificações de Williams et al. (1995); (6) Identificações de Phelps e Lada (1997), Román-Zúñiga (2006) e Poulton et al. (2008). Notas: (a) A0, $\mathrm{m}_{V}=9.9$; (b) $\mathrm{m}_{V}=11.4$; (c) $\mathrm{O} 8, \mathrm{~m}_{V}=8.2$; (d) $\mathrm{F} 2, \mathrm{~m}_{V}=9.4$; (e) $\mathrm{F} 5, \mathrm{~m}_{V}=7.6$; (f) $\mathrm{F} 6, \mathrm{~m}_{V}=$ 10.2; (g) Fonte com emissão em raios-X, do catálogo de Wang et al. (2009).

A correlação com os clumps identificados por Williams et al. (1995) é também destacada na Tabela 4.2. Nota-se que $1 / 3$ dos clumps selecionados aparecem fora das sub-regiões escolhidas em RMC. 


\subsection{Características das sub-regiões}

Cada uma das regiões selecionadas foi analisada de forma mais aprofundada através de imagens no infravermelho próximo, por meio de uma composição de imagens nas bandas $\mathrm{J}(1.25 \mu \mathrm{m}), \mathrm{H}(1.65 \mu \mathrm{m})$ e $\mathrm{K}(2.16 \mu \mathrm{m})$ do $2 M A S S$ dessas regiões, visando ilustrar como os YSOs se encontram associados com nebulosidades.

\subsubsection{Análise no infravermelho próximo para $R M C$}

As Figuras 4.10, 4.11, 4.12 e 4.13 mostram os resultados da composição das imagens nas bandas JHK, com campo de $\sim 17^{\prime} \times 8.5^{\prime}$, adotando-se níveis de cinza para melhor destacar as nebulosidades associadas às quatro regiões estudadas em RMC. Por outro lado, as imagens coloridas em RGB ( $\mathrm{J}$ - blue, H - green, $\mathrm{K}$ - red) mostradas nas Figuras 4.14, 4.15, 4.16 e 4.17 não permitem a visualização ressaltada de tais nebulosidades, dado seu menor contraste, porém são bastante úteis para revelar as características do conteúdo estelar. Neste caso, a emissão que aparece em vermelho indica a presença de material circunstelar (excesso na banda $\mathrm{K}$ ), enquanto que os objetos mais azulados representam a emissão estelar (maior emissão na banda J).

\subsubsection{Análise no infravermelho próximo para Mon R2}

As Figuras 4.18, 4.19, 4.20 e 4.21 apresentam as versões em tons de cinza, e as Figuras 4.22, 4.23, 4.24 e 4.25 são as composições RGB das imagens combinadas JHK, para Mon R2. Nota-se que o core de Mon R2 mostra claramente na Figura 4.18 subestruturas, enquanto na Figura 4.20 podemos ver a luz espalhada pelo disco de uma estrela T Tauri associada com a sub-região. É possivel verificar também que todas as imagens revelam excessos de cor típicos de associações jovens.

No próximo capítulo discutiremos as correlações encontradas na comparação entre a distribuição espacial dos objetos selecionados e suas características. 


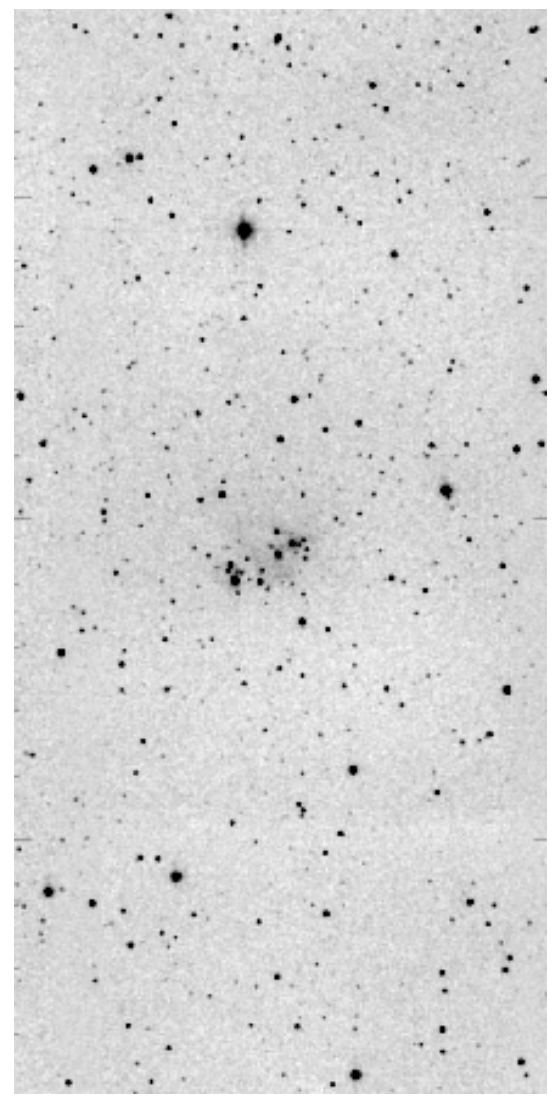

Figura 4.10: Composição das imagens JHK da sub-região A, em RMC, centrada em $\alpha=06: 31: 28$ e $\delta=04: 19: 06$.

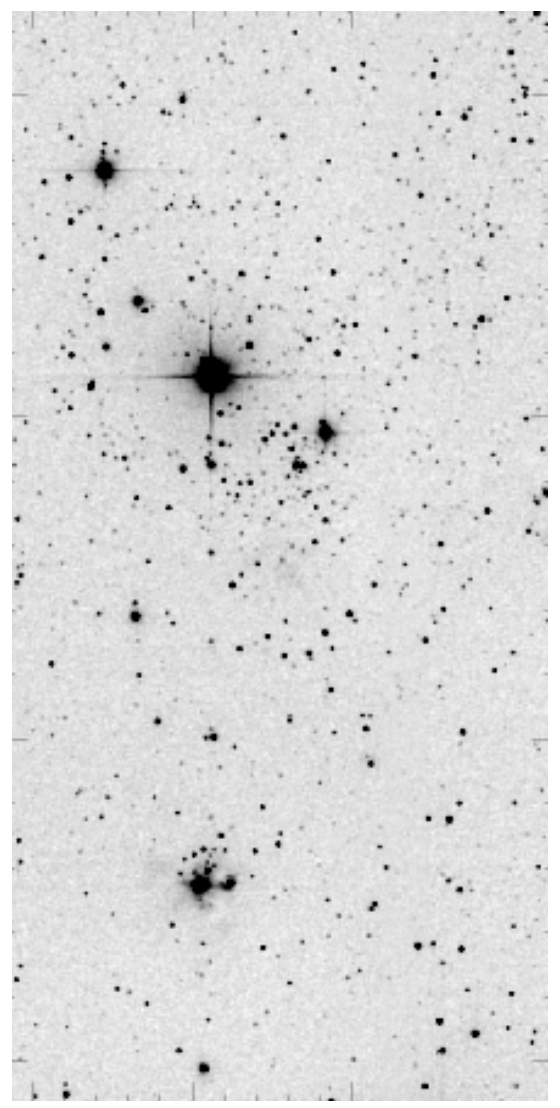

Figura 4.12: Idem à Figura 4.10, para a sub-região C, centrada em $\alpha=06: 34: 18$ e $\delta=04: 19: 52$.

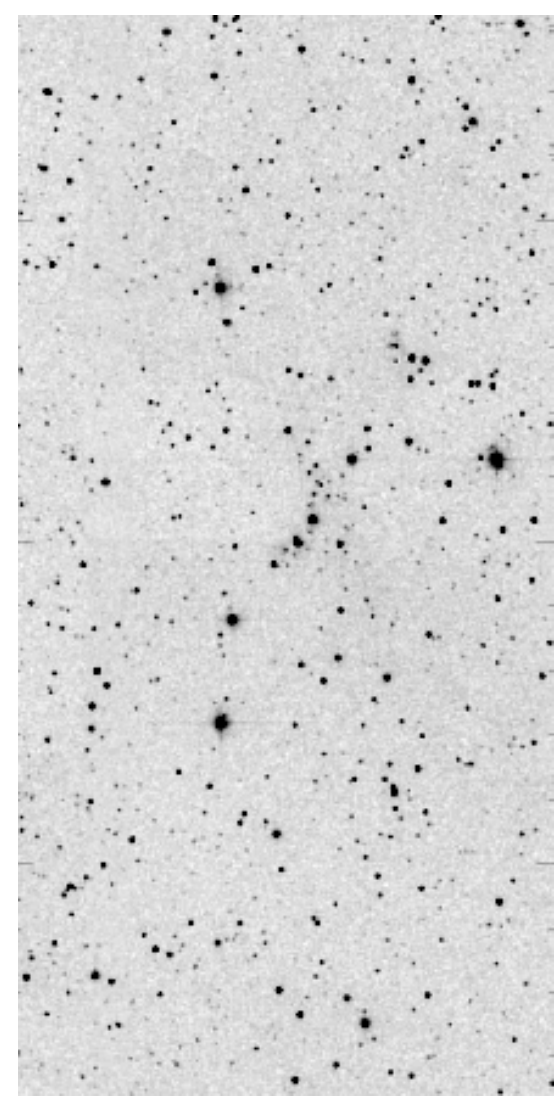

Figura 4.11: Idem à Figura 4.10, para a subregião $\mathrm{B}$, centrada em $\alpha=06: 33: 11$ e $\delta=04: 34: 41$.

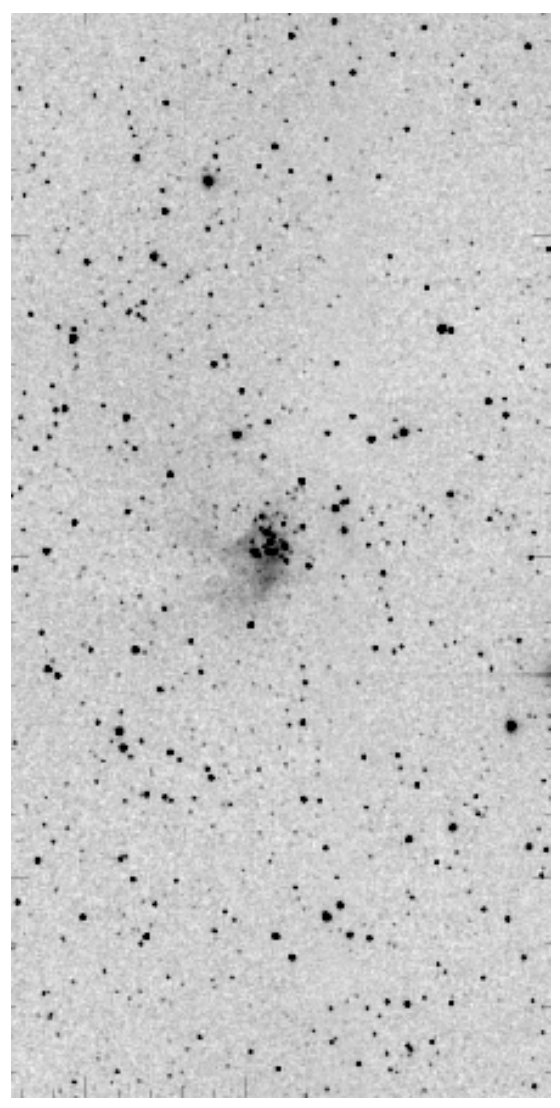

Figura 4.13: Idem à Figura 4.10, para a sub-região $\mathrm{D}$, centrada em $\alpha=06: 33: 49$ e $\delta=04: 30: 05$. 


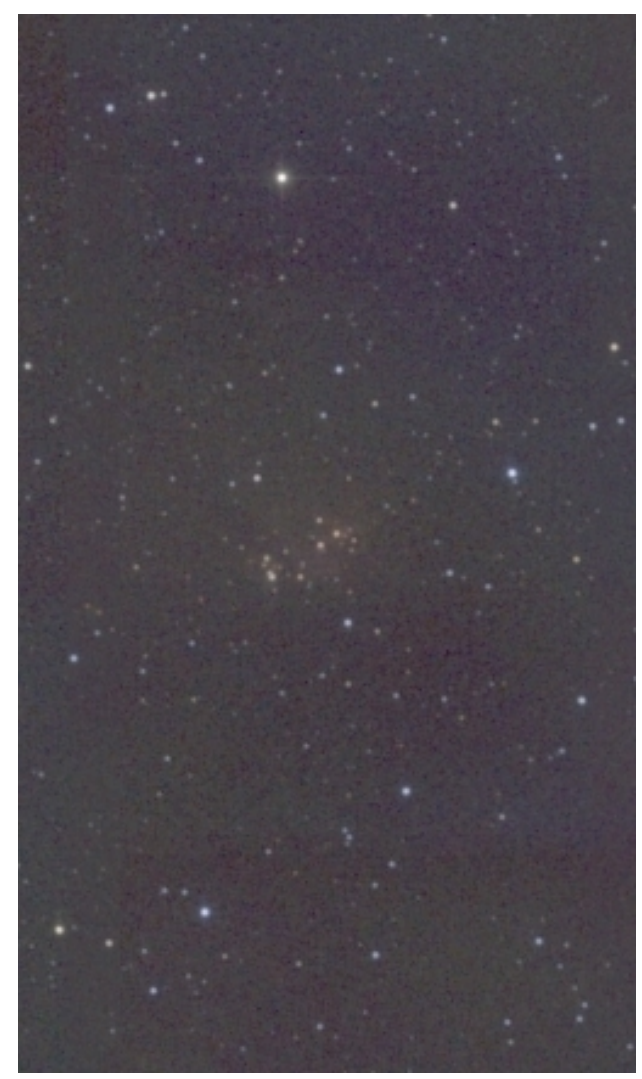

Figura 4.14: Composição RGB das bandas JHK da sub-região A, em RMC.

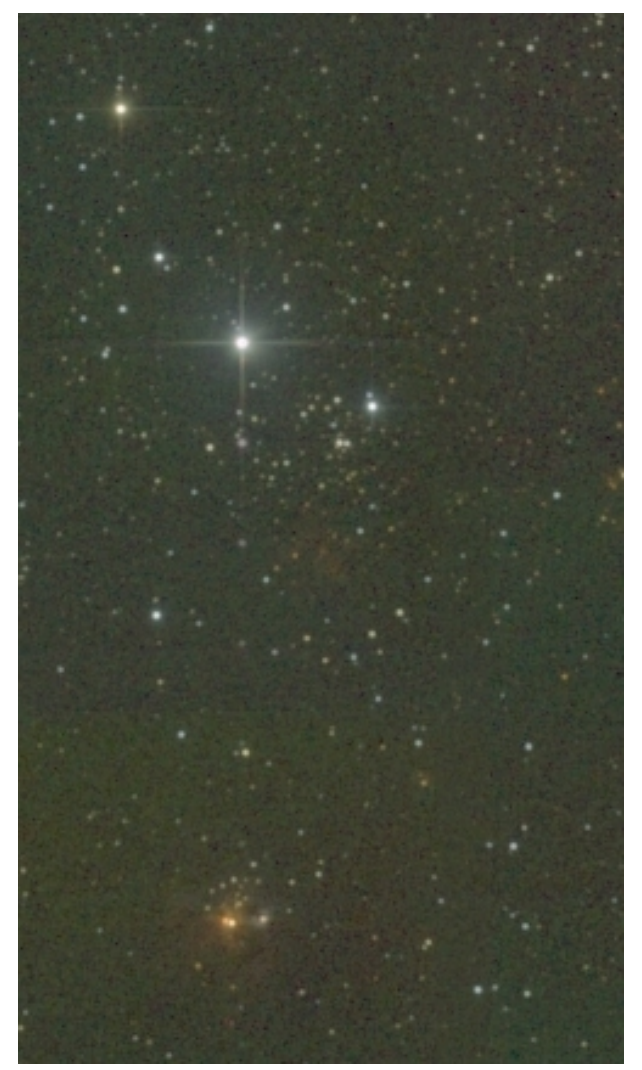

Figura 4.16: Idem à Fig. 4.14, para a sub-região C.

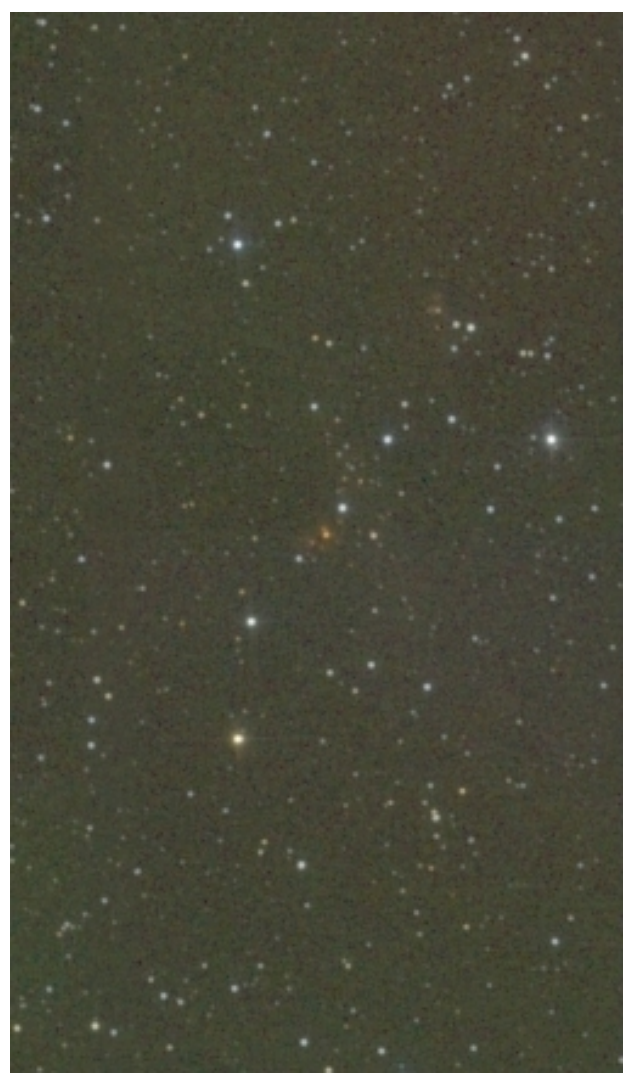

Figura 4.15: Idem à Fig. 4.14, para a sub-região B.

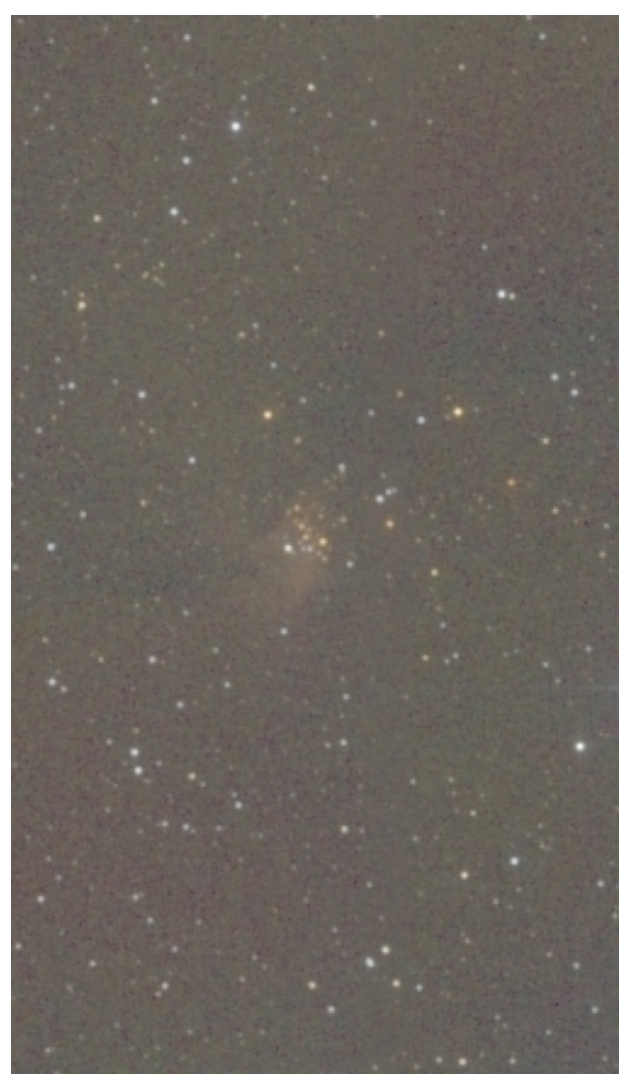

Figura 4.17: Idem à Fig. 4.14, para a sub-região D. 
Figura 4.18: Composição das imagens JHK do Core de Mon R2, centrado em $\alpha=06: 07: 48 \mathrm{e}$ $\delta=-06: 22: 20$, com campo de $\sim 5^{\prime} \times 5^{\prime}$.

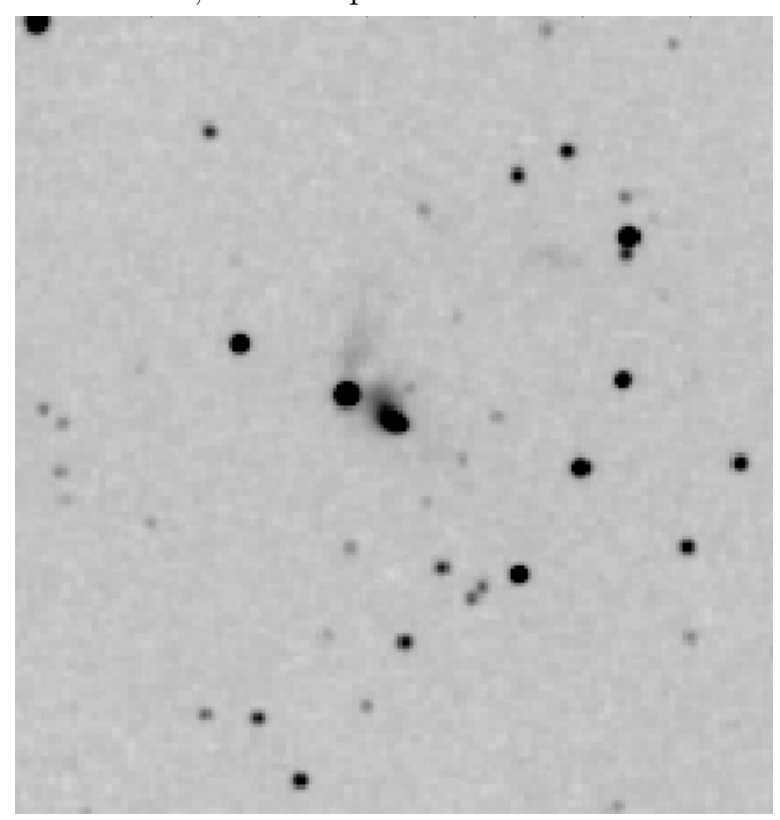

Figura 4.20: Idem à Figura 4.18, para GGD 16-17, centrada em $\alpha=06: 12: 48$ e $\delta=-06: 13: 56$, com campo de $\sim 4^{\prime} \times 4^{\prime}$.

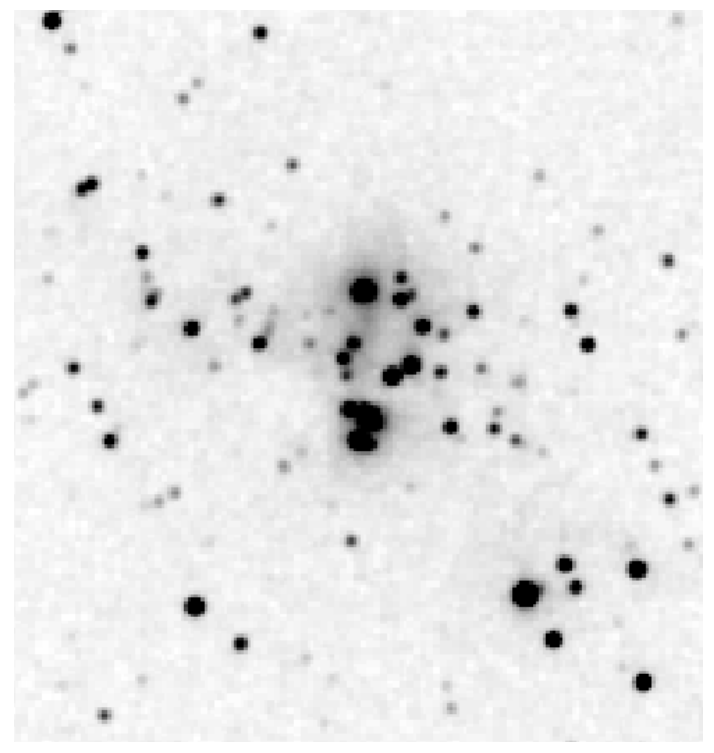

Figura 4.19: Idem à Figura 4.18, para GGD 12-15, centrada em $\alpha=06: 10: 49$ e $\delta=-06: 11: 38$, com campo de $\sim 4^{\prime} \times 4^{\prime}$.

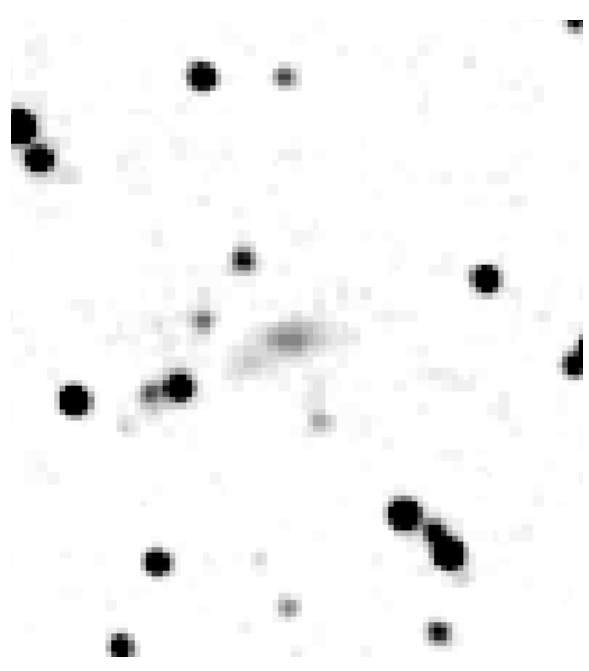

Figura 4.21: Idem à Figura 4.18, para HH866, centrada em $\alpha=06: 07: 08$ e $\delta=-06: 03: 53$, com campo de $\sim 2^{\prime} \times 2^{\prime}$. 


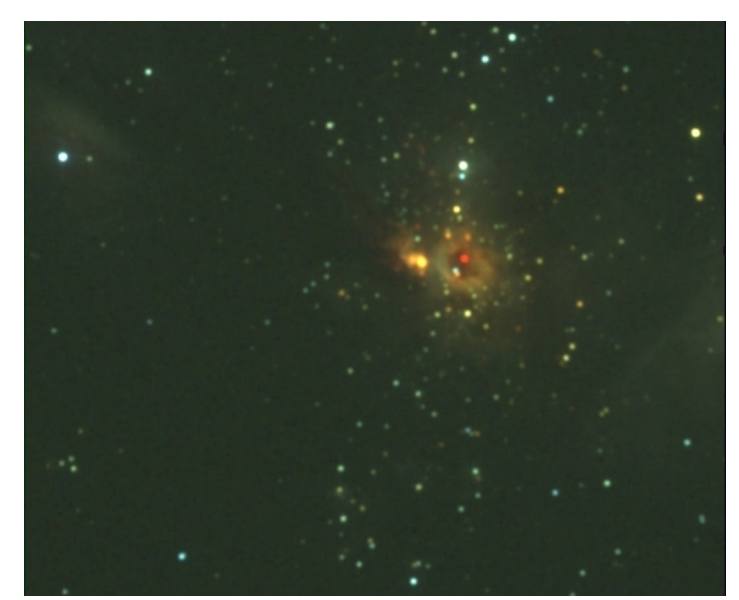

Figura 4.22: Composição RGB das bandas JHK do core central de Mon R2.

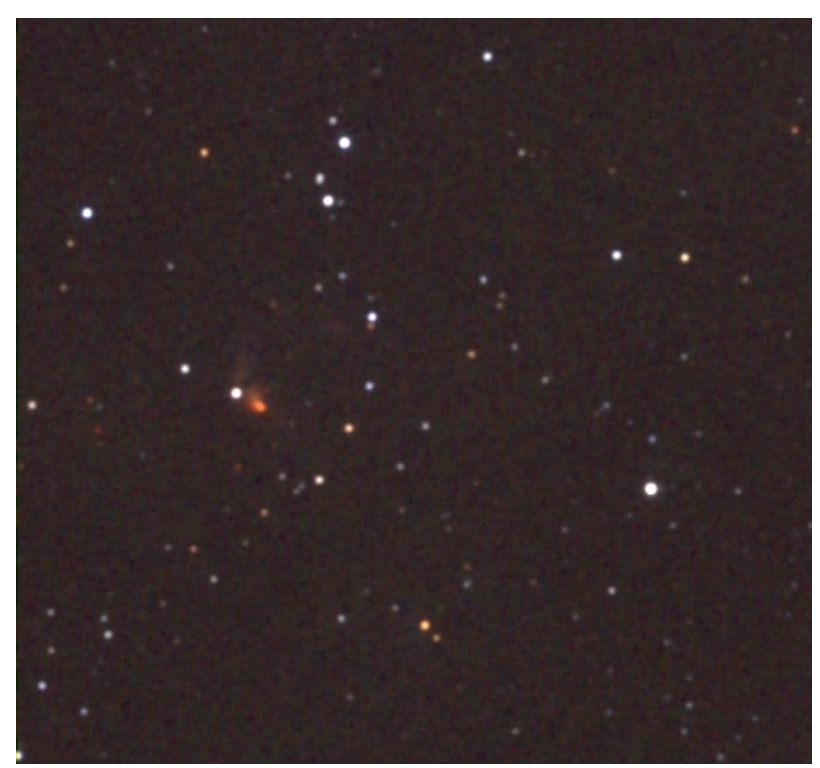

Figura 4.24: Idem à Fig. 4.22, para GGD 16-17.

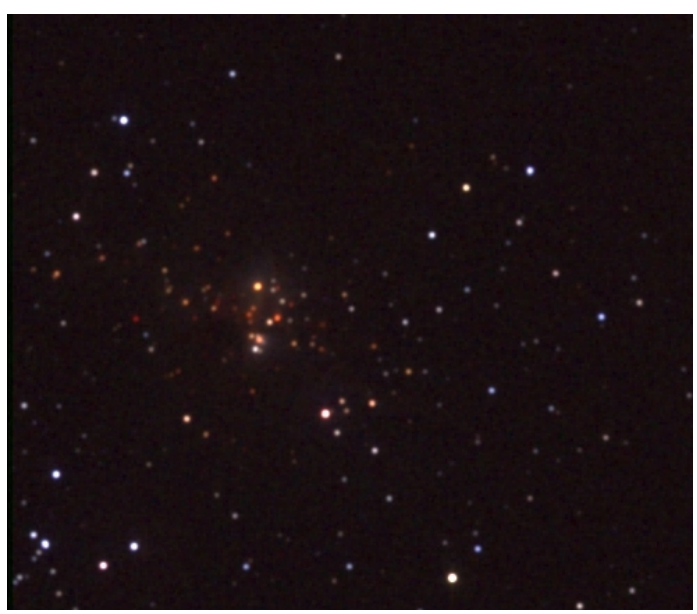

Figura 4.23: Idem à Fig. 4.22, para GGD 1215.

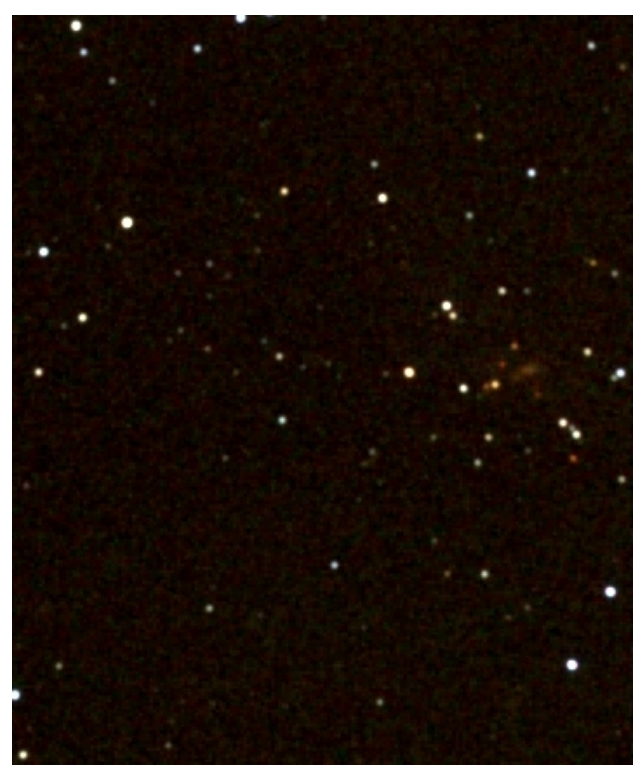

Figura 4.25: Idem à Fig. 4.22, para HH866. 
Capítulo 5

\section{Discussão dos Resultados}

As Figuras 5.1 e 5.2 apresentam, respectivamente para RMC e Mon R2, o quadro global de todos os mapas obtidos no presente trabalho e destacam as sub-regiões avaliadas desde as maiores escalas (mapa de CO comparado ao mapa de extinção), passando para média escala (contornos de fluxo IRAS em $100 \mu \mathrm{m}$, combinados com emissão de CO e imagem óptica), chegando a escalas menores, apresentadas nas imagens $2 M A S S$, compostas nas bandas JHK, para as sub-regiões de interesse.

Neste capítulo, inicialmente discutimos e comparamos as semelhanças e diferenças encontradas entre as nuvens aqui estudadas. Ao final, confrontamos as características de nossos alvos com as demais GMCs encontradas no Complexo de Monoceros e sua contribuição para melhor compreender o cenário de formação estelar nessa região. 


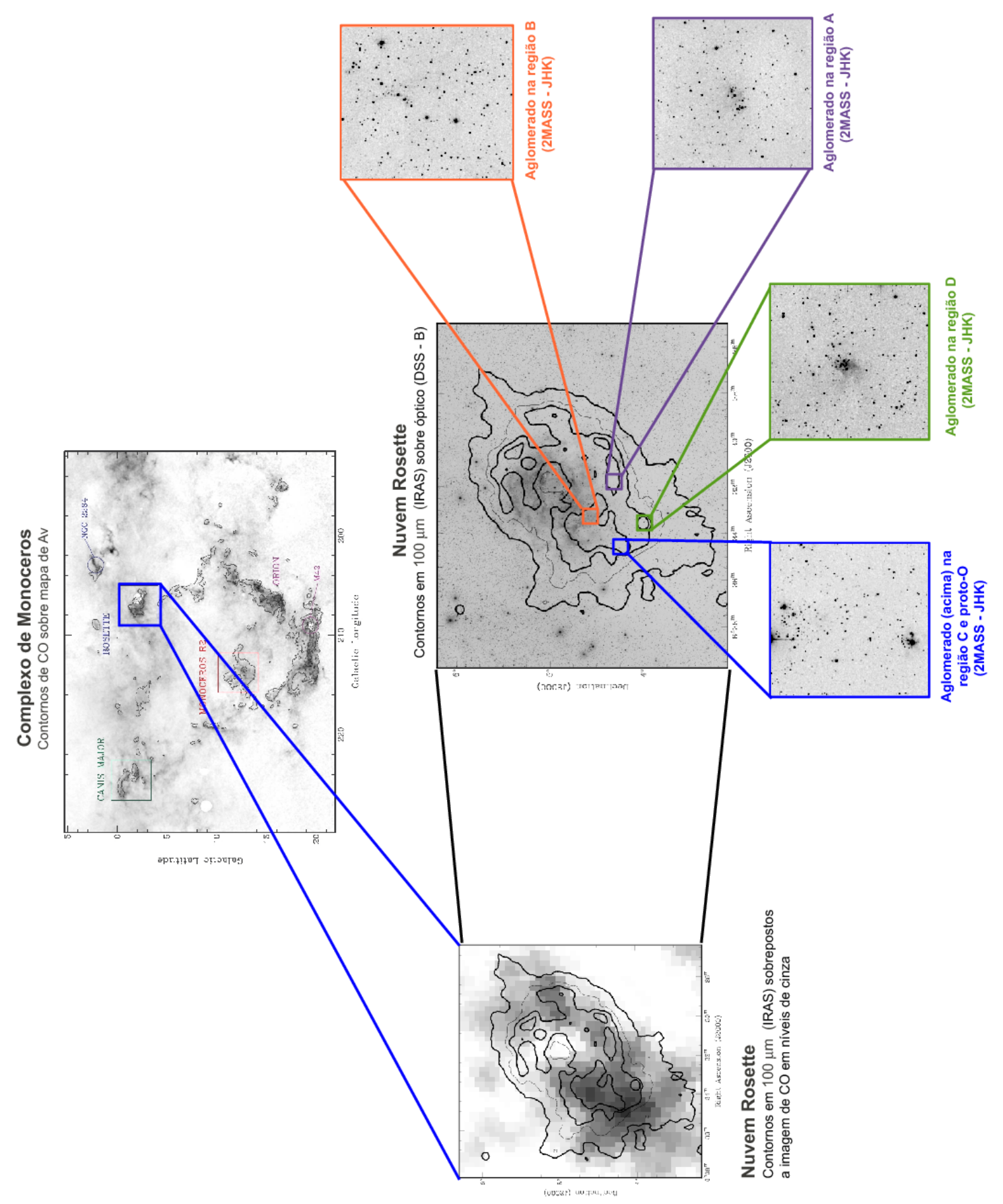

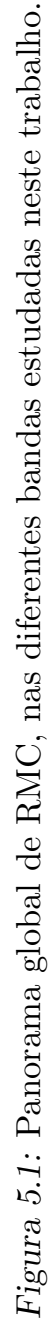




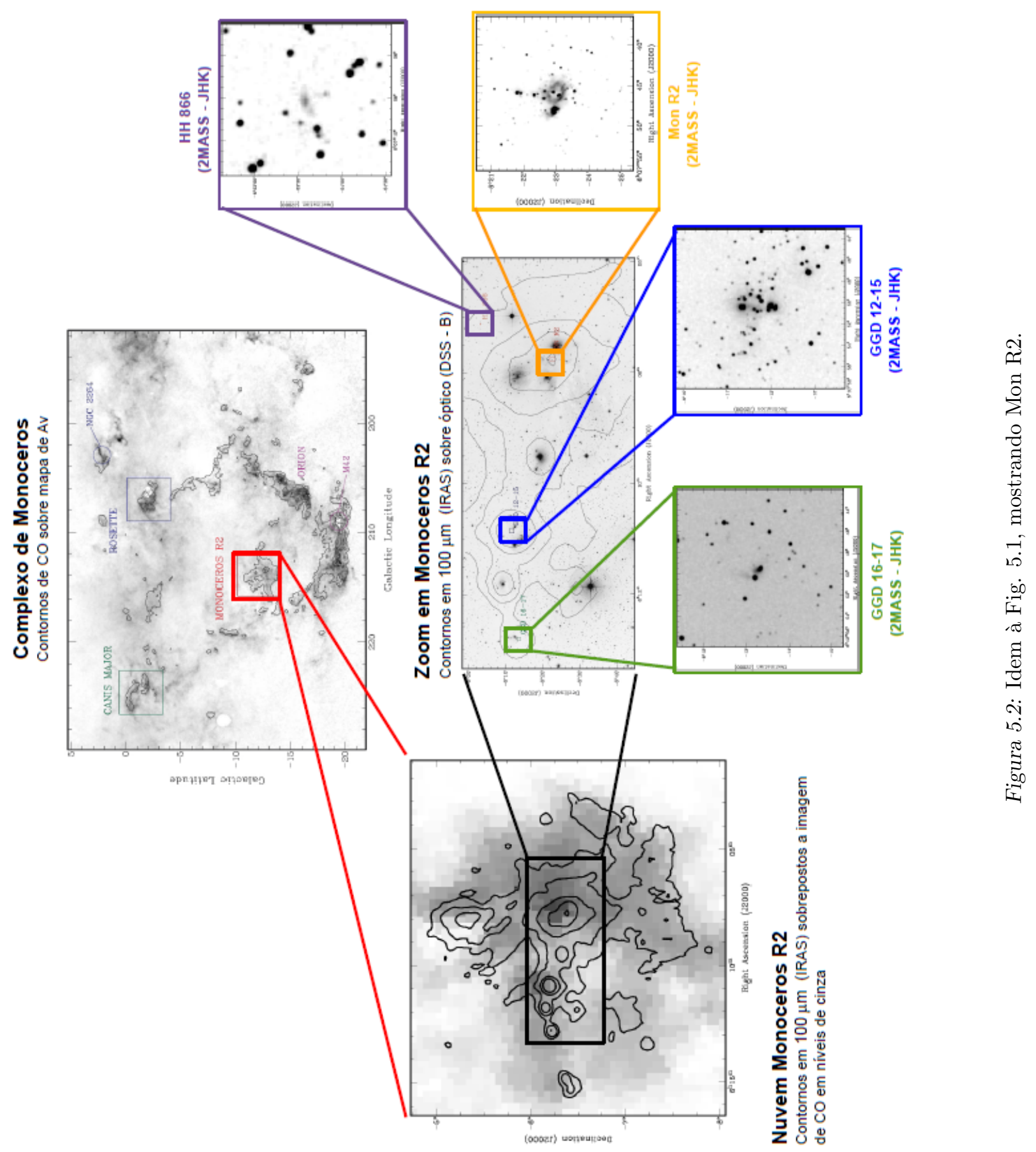




\subsection{Estrutura das nuvens}

A primeira semelhança que se nota ao comparar a distribuição de gás e poeira das nuvens estudadas é a coincidência entre os picos de intensidades dos diversos traçadores. Os contornos de $\mathrm{CO}$ apresentados na Figura 4.2 indicam uma boa correlação com a imagem de fundo, que revela as regiões com maior concentração de poeira, indicadas pelo mapa de extinção. Por outro lado, enquanto RMC apresenta uma distribuição aproximadamente uniforme de $\mathrm{A}_{V}$, Mon R2 mostra uma estrutura mais filamentária, semelhante ao que é observado em CMa R1, e também muito parecida com a distribuição espacial da extinção em Orion.

De uma forma geral, os níveis de extinção - bem como os picos de densidade CO são muito mais elevados em Orion do que nas outras regiões, exceto pelo core de Mon R2. Apenas uma sub-região entre as demais estudadas pode ser considerada semelhante às distribuições de gás e poeira encontradas em Orion.

Os mapas no infravermelho distante também mostram-se bem correlacionados com a distribuição de gás molecular, medida pela emissão de $\mathrm{CO}$ em ambas as nuvens, como pode ser observado nas Figuras 4.3 e 4.4. Nota-se, nessas imagens sobrepostas, a ausência de uma melhor resolução espacial nos mapas de CO disponíveis para nossas regiões. No entanto, a boa correlação indicada com a emissão em $100 \mu \mathrm{m}$, pelo menos nas regiões centrais de RMC e Mon R2, propicia um estudo indireto da distribuição do gás por meio dos mapas nas bandas $I R A S$, que tem uma resolução um pouco melhor (1.5 arcmin/pixel) que a dos mapas de $\mathrm{CO}(\sim 5 \mathrm{arcmin} / \mathrm{pixel})$.

Nesse sentido, optamos por explorar a distribuição da razão de fluxos IRAS nas duas bandas que permitem separar a emissão estelar da interestelar, ou seja em 60 e $100 \mu \mathrm{m}$ (Bloemen et al., 1990). Ao se comparar a distribuição de nuvens escuras (mapa de $\mathrm{A}_{V}$ ) com a razão de fluxos $\mathrm{F}_{60} / \mathrm{F}_{100}$ na imagem da Figura 4.5, nota-se que a emissão estelar tende a estar próxima das nuvens, porém não necessariamente projetada contra as nuvens escuras de fundo. Isso pode ser visto pela distribuição dos níveis de contornos sobrepostos ao mapa de extinção correspondem a $\mathrm{F}_{60} / \mathrm{F}_{100}=0.25$, o limite que adotamos para separar a emissão interestelar $\left(\mathrm{F}_{100}>4 \mathrm{~F}_{60}\right)$, de acordo com o verificado para as estrelas coincidentes com as regiões estudadas (vide Sec. 5.3).

Desta forma, podemos diferenciar a emissão estelar de objetos associados às nuvens 
(pela correlação entre valores de $\mathrm{A}_{V}$ e $\mathrm{F}_{60} / \mathrm{F}_{100}$ ) e separar os objetos não associados. Portanto, a emissão estelar nem sempre coincide com as áreas de maior extinção (objetos altamente embebidos, ou ausência de formação estelar nessas nuvens), assim como ocorre emissão estelar em regiões de baixa extinção (estrelas recém-formadas que consumiram o material a seu redor, ou eventuais estrelas de campo). Para demonstrar essas diferenças e semelhanças, na Figura 5.3 apresentamos um gráfico que correlaciona a extinção visual medida nos mapas de Dobashi et al. (2005) com a razão de fluxos $I R A S \mathrm{~F}_{60} / \mathrm{F}_{100}$, obtida para os masers encontrados nas regiões de nosso interesse. Em RMC nota-se que os masers tendem a apresentar maiores valores de extinção $\left(\mathrm{A}_{V}>1.5 \mathrm{mag}\right)$ enquanto que em Mon $\mathrm{R} 2$, os valores de $\mathrm{A}_{V}$ são levemente menores. Por outro lado, não se verifica uma tendência particular quando se compara a extinção com a razão de fluxos IRAS. Uma anticorrelação entre $A_{V}$ e $F_{60} / F_{100}$ seria esperada para representar a predominância da emissão interestelar, porém em nossa amostra de masers somente um dos objetos (IRAS 06056-0700) apresenta essa característica $\left(\mathrm{A}_{V}>1.5 \mathrm{mag}\right.$ e $\left.\mathrm{F}_{60} / \mathrm{F}_{100}<0.25\right)$, indicando um grau de embebimento possivelmente maior do que nos outros casos.

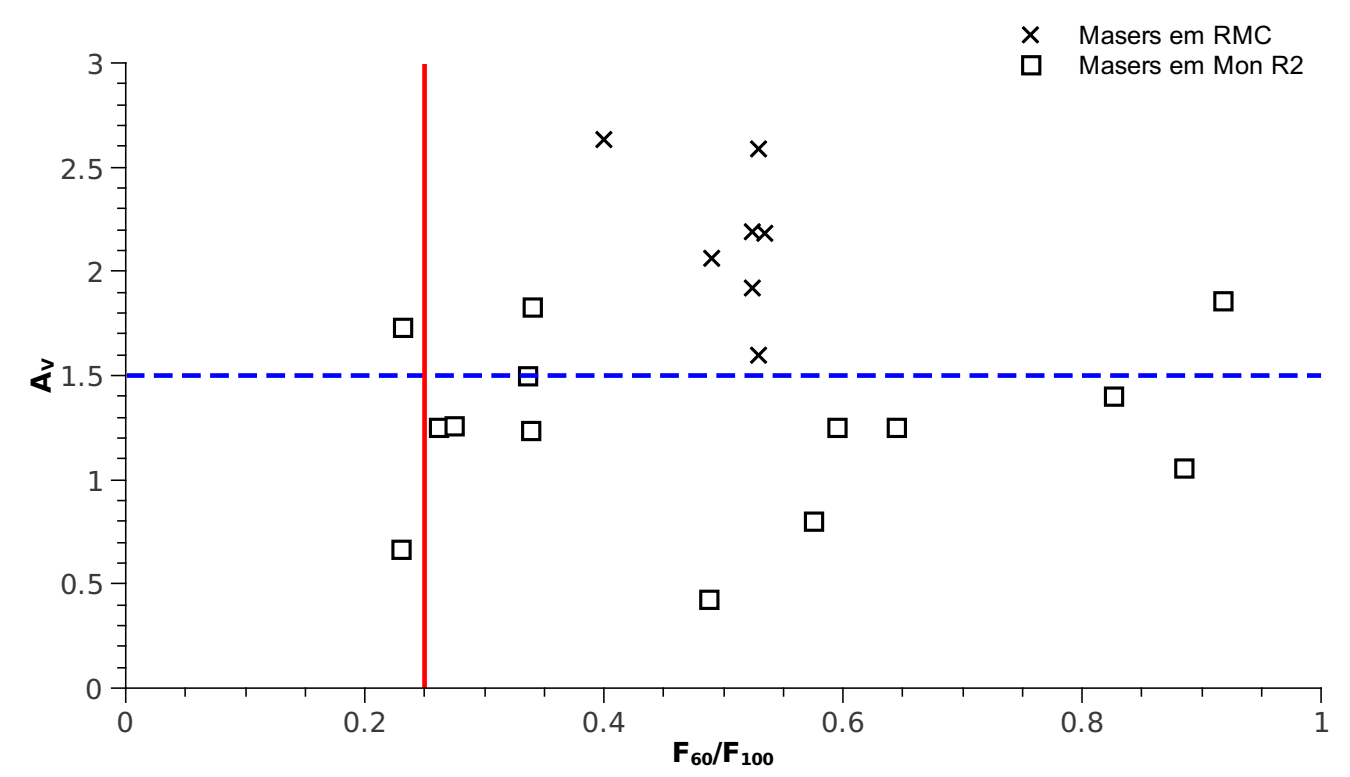

Figura 5.3: Extinção visual medida nos mapas de (Dobashi et al., 2005) comparada com a razão de fluxos IRAS $\mathrm{F}_{60} / \mathrm{F}_{100}$ obtida para os masers encontrados em RMC e Mon R2. A linha azul tracejada delimita altas extinções $\left(\mathrm{A}_{V}>1.5\right)$ e a linha vermelha indica predominância de emissão estelar $\left(\mathrm{F}_{60} / \mathrm{F}_{100}<0.25\right)$.

Ao se comparar a intensidade da emissão maser $\mathrm{H}_{2} \mathrm{O}$ com a razão de fluxos IRAS 


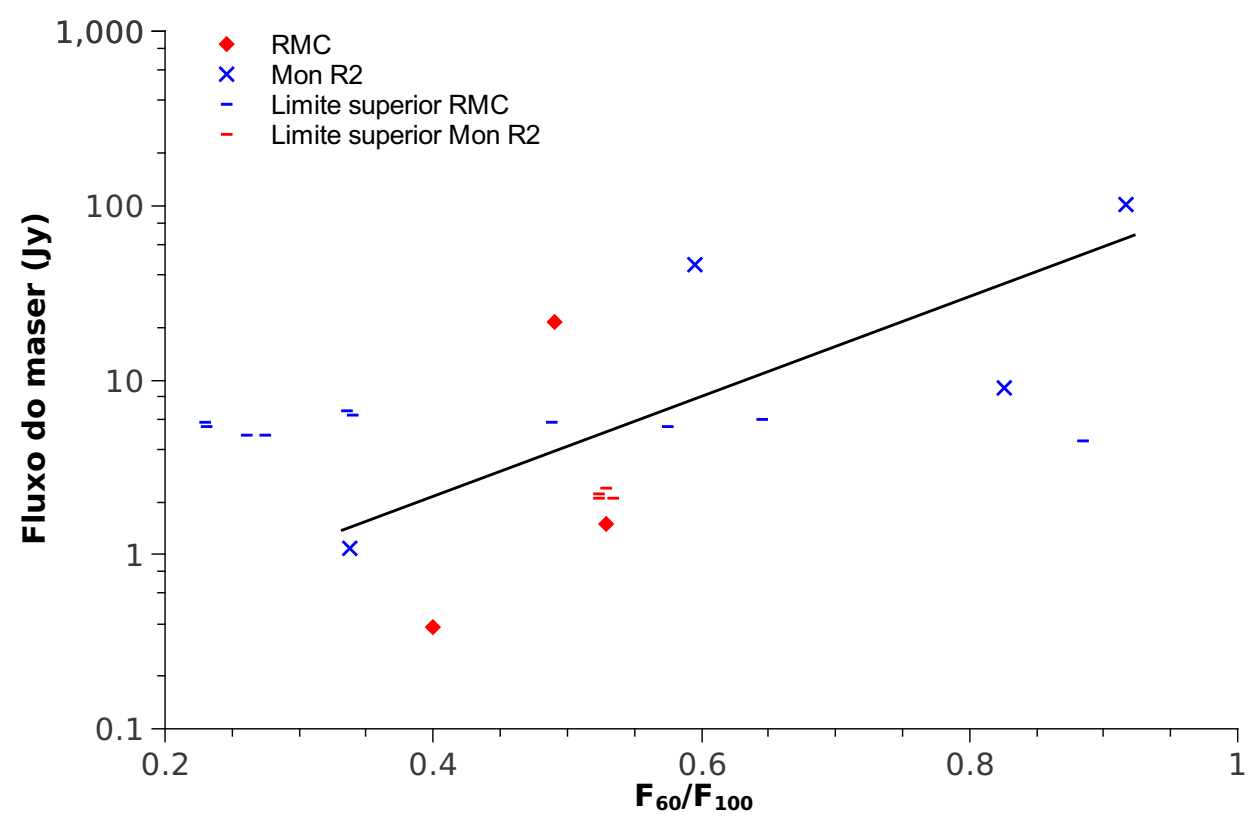

Figura 5.4: Comparação do fluxo de emissão maser $\mathrm{H}_{2} \mathrm{O}$ com a razão de fluxos IRAS $\mathrm{F}_{60} / \mathrm{F}_{100}$, obtida para objetos encontrados em RMC e Mon R2.

$\mathrm{F}_{60} / \mathrm{F}_{100}$ notamos uma tendência, pelo menos para os fluxos com melhor qualidade de medida (indicada pela linha cheia na Fig. 5.4). Ou seja, os masers melhor detectados e mais intensos tendem a apresentar uma maior razão $\mathrm{F}_{60} / \mathrm{F}_{100}$. No entanto, são poucas as medidas utilizadas para verificar essa tendência, já que os outros obje- tos estudados (com limite superior na medida do fluxo maser) apresentam-se distribuídos aleatoriamente no diagrama ilustrado na Figura 5.4. 


\subsection{Características dos clumps}

Com base nos dados fornecidos na Tabela 2.5 foi possível avaliar algumas características relacionadas à distribuição de densidades em RMC. Resultados interessantes foram obtidos na comparação entre extinção visual e parâmetros físicos dos clumps, tais como dimensão e temperatura, por exemplo, como descrevemos a seguir.

- Extinção visual em função da dimensão do clump: Apesar da alta dispersão, notase uma tendência de aumento da extinção com o aumento da dimensão do clump, como mostra a Figura 5.5. A extinção traça a distribuição de poeira e, dessa forma, fornece uma estimativa da densidade colunar do hidrogênio. A distribuição de poeira também traz informações sobre o processo de fragmentação, que pode ser responsável pela forma da função inicial de massa através de movimentos turbulentos no meio interestelar (Cambrésy et al., 2002).

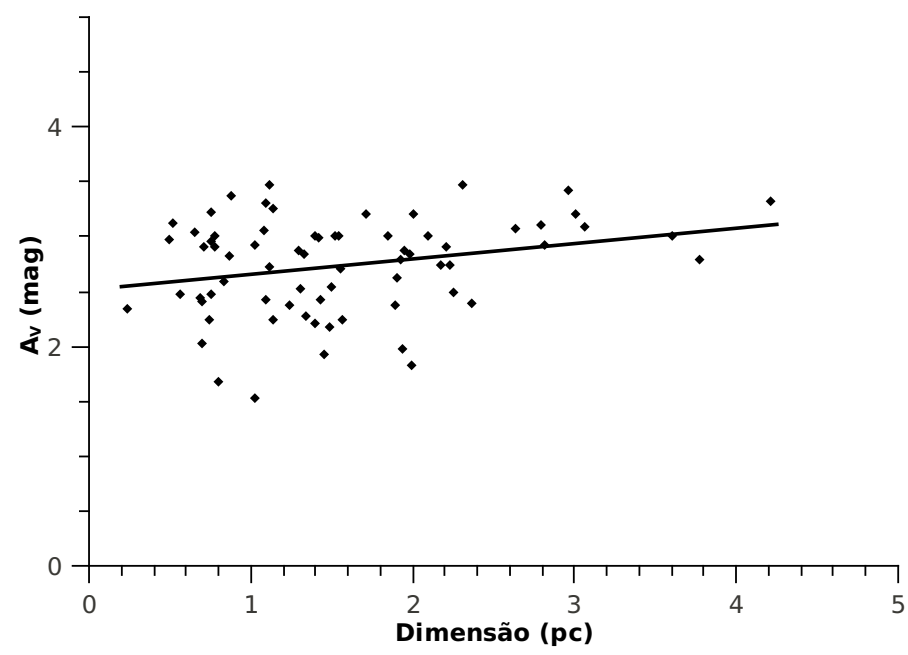

Figura 5.5: Extinção visual dos clumps localizados em RMC em função de sua dimensão. A linha cheia indica a tendência aproximada.

- Extinção visual em função da temperatura externa do clump: Essa correlação é devida ao processo de condensação do fragmento pois, quanto maior a extinção do clump, mais concentrado ele é e menor será sua temperatura devido à concentração de poeira (fria). Como podemos verificar na Figura 5.6, a extinção visual tende a decrescer com o aumento da temperatura externa do clump. 


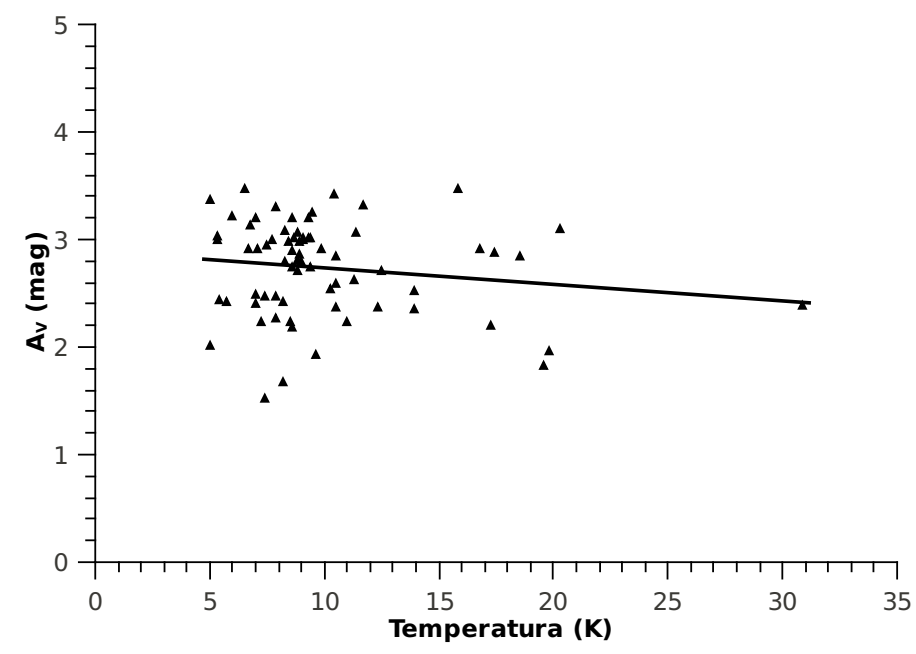

Figura 5.6: Idem à Fig. 5.5, mostrando extinção visual em função da temperatura externa do clump.

- Temperatura externa do clump em função de sua dimensão: Na Figura 5.7 notase uma tendência de clumps de maior dimensão apresentarem maiores temperaturas. Porém, como nos casos anteriores, a alta dispersão não permite resultados conclusivos a respeito da correlação entre as características dos clumps.

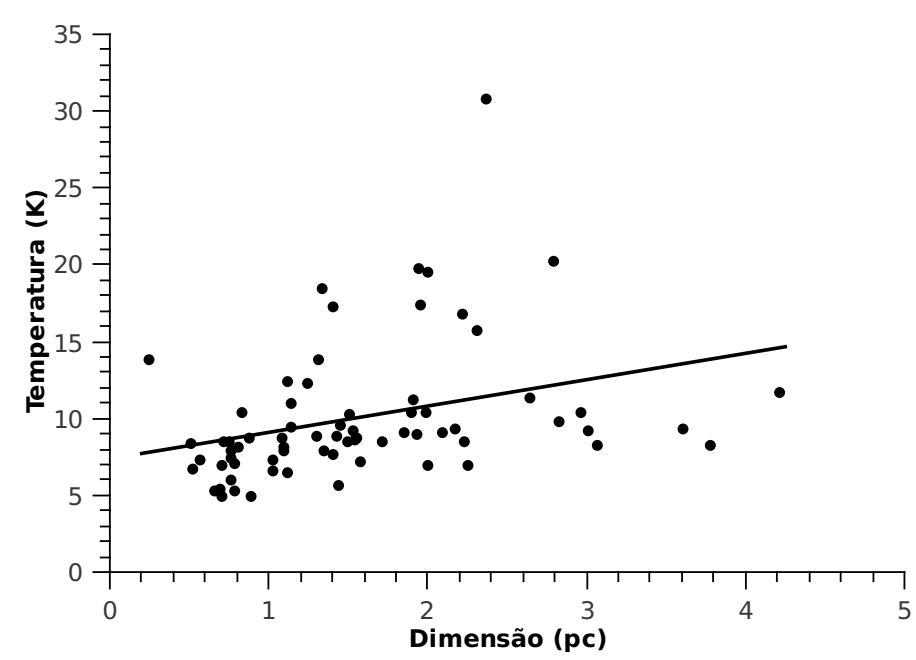

Figura 5.7: Idem à Fig. 5.5, mostrando temperatura externa do clump em função de sua dimensão. 
Infelizmente não há para Mon R2 a disponibilidade dos mesmos dados, para realizarmos uma comparação entre nuvens. Porém, Tafalla et al. (1994) detectaram alguns clumps na parte central de Mon R2. As características desses fragmentos de nuvem estão apresentadas na Tabela 5.1, sendo identificados como componentes primárias de velocidade (B, C e G, por exemplo) e componentes secundárias (B', C' e G', nesse caso), sugerindo a presença de mais de um clump na mesma posição. A localização dos clumps é descrita em relação ao máximo no contínuo de rádio, em $6 \mathrm{~cm}$.

Tabela 5.1 - Parâmetros de clumps localizados em Mon R2. Tabela baseada em Tafalla et al. (1994).

\begin{tabular}{cccccc}
\hline \hline Clump & $\begin{array}{c}\Delta \alpha(1) \\
(\operatorname{arcsec})\end{array}$ & $\begin{array}{c}\Delta \delta^{(1)} \\
(\operatorname{arcsec})\end{array}$ & $\begin{array}{c}\mathrm{V}_{L S R} \\
\left(\mathrm{~km} \mathrm{~s}^{-1}\right)\end{array}$ & $\begin{array}{c}\mathrm{n}_{H_{2}} \\
\left(\mathrm{~cm}^{-3}\right)\end{array}$ & $\begin{array}{c}\mathrm{M} \\
\left(\mathrm{M}_{\odot}\right)\end{array}$ \\
\hline $\mathrm{A}$ & -120 & 100 & 6.5 & $6.0 \times 10^{5}$ & 3.7 \\
$\mathrm{~B}$ & -140 & 40 & 4.5 & $7.5 \times 10^{5}$ & 11.2 \\
$\mathrm{~B}$, & -140 & 40 & 8.2 & $1.3 \times 10^{5}$ & 23.9 \\
$\mathrm{C}$ & -20 & 20 & 7.2 & $2.5 \times 10^{5}$ & 21.1 \\
$\mathrm{C}$ & 0 & 40 & 8.4 & $6.5 \times 10^{5}$ & 5.7 \\
$\mathrm{D}$ & -120 & -20 & 9.0 & $2.7 \times 10^{5}$ & 9.1 \\
$\mathrm{E}$ & -100 & -40 & 13.1 & $4.7 \times 10^{5}$ & 4.3 \\
$\mathrm{~F}$ & -40 & -140 & 13.4 & $2.8 \times 10^{5}$ & 11.2 \\
$\mathrm{G}$ & 60 & -80 & 15.6 & $2.1 \times 10^{5}$ & 6.0 \\
$\mathrm{G}$ & 20 & -60 & 13.0 & $3.3 \times 10^{5}$ & 2.3 \\
$\mathrm{H}$ & -40 & -80 & 13.2 & $1.8 \times 10^{5}$ & $\ldots$ \\
\hline (1) Offset & referente a $\alpha(1950)=06^{h} 05^{m} 20^{s}, \delta=-06^{\circ} 22^{\prime} 39.4^{\prime \prime}$.
\end{tabular}




\subsection{Distribuição espacial da população estelar}

A presença de estrelas massivas (tipo O) em RMC, concentradas no centro da nebulosa NGC2244, é uma importante diferença com relação a Mon R2, que tem apenas estrelas do tipo B, cuja distribuição segue a estrutura filamentária observada em $\mathrm{CO}$, enquanto que em RMC as estrelas B aparecem distribuídas uniformemente.

As Figuras 4.6 e 4.7 mostram imagens ópticas sobrepostas por contornos dos mapas IRAS, que permitem comparar a posição das estrelas, associadas às nuvens, com relação à distribuição de poeira (emissão em $100 \mu \mathrm{m})$.

Exceto pela notável Nebulosa Rosette, não ocorrem outras nebulosidades de destaque em RMC (Fig. 4.6), sendo que todas as estrelas massivas encontram-se na parte central da região HII (NGC2244). Por outro lado, em Mon R2 várias nebulosas de reflexão são encontradas, sempre associadas a regiões de maior concentração de poeira. Na Figura 4.7 notam-se seis picos de emissão em $100 \mu \mathrm{m}$, nos quais encontram-se pelo menos oito nebulosidades, entre elas destacam-se três das regiões estudadas por nós em maior detalhe (core de Mon R2, GGD12-15 e GGD16-17). Neste caso, a região onde se encontra o objeto HH866 é a única que não contém nebulosidades e nem coincide com alta concentração de poeira.

Nas Tabelas 4.2 e 4.3 correlacionamos os objetos listados em diferentes catálogos e que se apresentam aproximadamente coincidentes em termos de distribuição espacial. Essa distribuição é ilustrada nas Figuras 4.8 e 4.9 , respectivamente para RMC e Mon R2. O painel com a imagem em maior escala (mapa em $100 \mu \mathrm{m}$ ) nessas figuras mostra uma distribuição da população que segue a mesma estrutura filamentária da nuvem em Mon R2, semelhante ao que foi discutido na Sec. 5.1 com relação ao mapa de CO. O painel em zoom, da Figura 4.9 apresenta a emissão estelar (mapa $\mathrm{F}_{60} / \mathrm{F}_{100}$ ), com uma razoável coincidência na posição entre fontes IRAS, masers, raios-X e suas contrapartidas ópticas. São listadas 25 coincidências para Mon R2 na Tabela 4.3 , enquanto que para RMC esse número cai para 18. Em termos de densidade superficial de objetos tais diferenças são ainda maiores, considerando a proporcionalidade das áreas envolvidas, que no caso de Mon R2 é cerca de duas vezes menor que RMC. Desta forma, a densidade de objetos selecionados em Mon R2 é cerca de cinco vezes maior que em RMC, apesar de os critérios de seleção terem sido os mesmos. Isso indica para Mon R2 uma população jovem mais rica que em RMC, porém não 
necessariamente mais jovem. Uma forma de comparar as características das populações estelares dessas regiões é por meio da função de luminosidade em raios-X $\left(\mathrm{L}_{X}\right)$, obtida com dados ROSAT por Gregorio-Hetem et al. (1998). As Figuras 5.8 e 5.9 apresentam a distribuição integrada (para Mon $\mathrm{R} 2$ e RMC, respectivamente) de $\mathrm{N}\left(\mathrm{L}_{X}\right)$ vs. $\mathrm{L}_{X}$, onde $\mathrm{N}\left(\mathrm{L}_{X}\right)$ corresponde ao número total (normalizado) de fontes com luminosidade maior que $\mathrm{L}_{X}$. Esses diagramas foram apresentados por Gregorio-Hetem (2003) visando comparar as características das fontes-X detectadas em Mon R2 e RMC, com aquelas detectadas nas nuvens moleculares Cha 1, que contém objetos com idades da faixa de $10^{7}$ anos, e $\rho$ Oph cujos membros têm idades da ordem de $10^{6}$ anos.

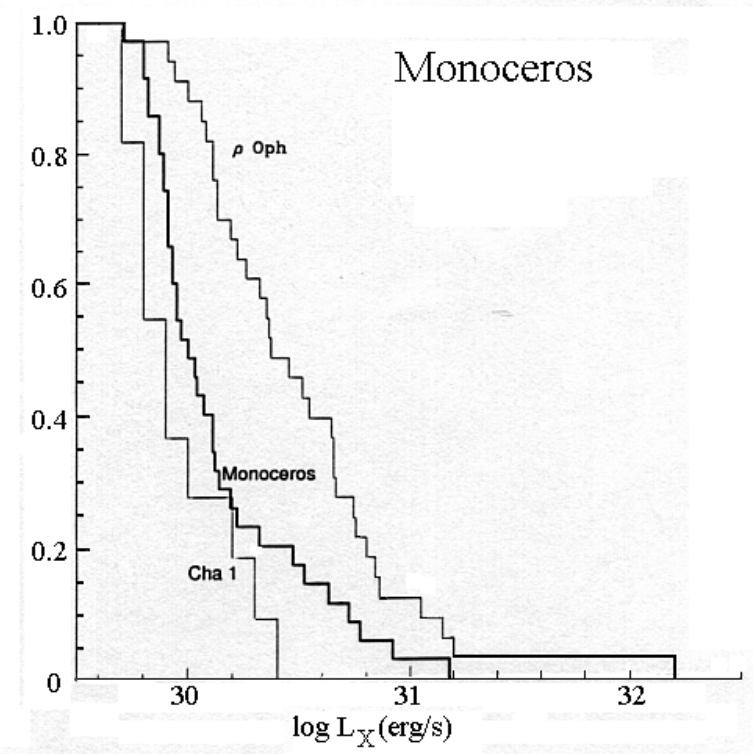

Figura 5.8: Função de luminosidade X integrada e normalizada para Mon R2, comparada com as nuvens Cha 1 e $\rho$ Oph. Figura de Gregorio-Hetem (2003).

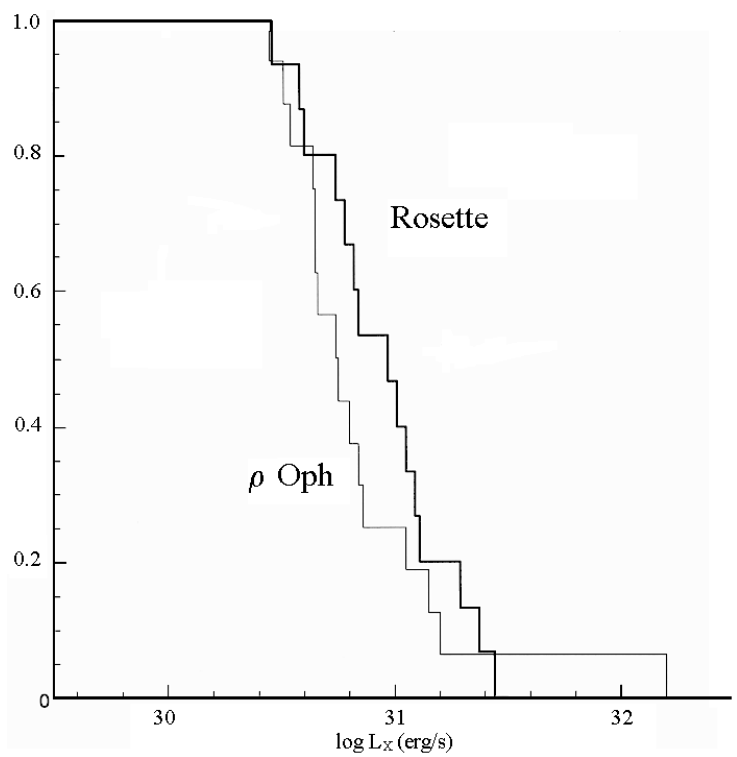

Figura 5.9: Idem à Fig. 5.8. No caso de Rosette, a comparação é feita somente com a nuvem $\rho$ Oph, mostrando-se bastante diferente da $\mathrm{F}\left(\mathrm{L}_{X}\right)$ de Mon R2.

O resultado encontrado para Mon R2 é de uma função de luminosidade X intermediária entre as duas nuvens de comparação, mas com tendência mais próxima à de Cha 1. No caso de RMC, a função de luminosidade tem uma distribuição superior à de $\rho$ Oph, o que pode ser um indicativo de uma maior quantidade de objetos mais jovens. No entanto, as amostras detectadas pelo ROSAT não são tão completas, em particular para objetos de baixas massas, quanto aquelas detectadas pelo XMM e pelo Chandra, por exemplo. Assim, a comparação das funções de luminosidade-X deve ser avaliada com cautela, servindo apenas como um indicativo das diferenças entre as nuvens, quando se trata de uma análise 
sobre uma mesma base de dados, neste caso, resultados do ROSAT obtidos para as quatro nuvens apresentados nas Fig. 5.8 e 5.9.

\subsection{Estágio evolutivo das fontes IRAS}

Codella e colaboradores desenvolveram uma série de trabalhos relacionados à natureza de protoestrelas massivas e o estágio evolutivo de masers de $\mathrm{H}_{2} \mathrm{O}$ (Codella e Felli, 1995; Codella et al., 1995; Codella e Palla, 1995). Eles utilizam as cores IRAS [25-12] e [6012], definidas como $[\mathrm{i}-\mathrm{j}]=\log \left[\mathrm{F}_{i} / \mathrm{F}_{j}\right]$, para selecionar candidatas a UC HII (regiões HII ultra compactas) e separar objetos em diferentes fases evolutivas, identificando possíveis protoestrelas massivas (precursoras de UCHII).

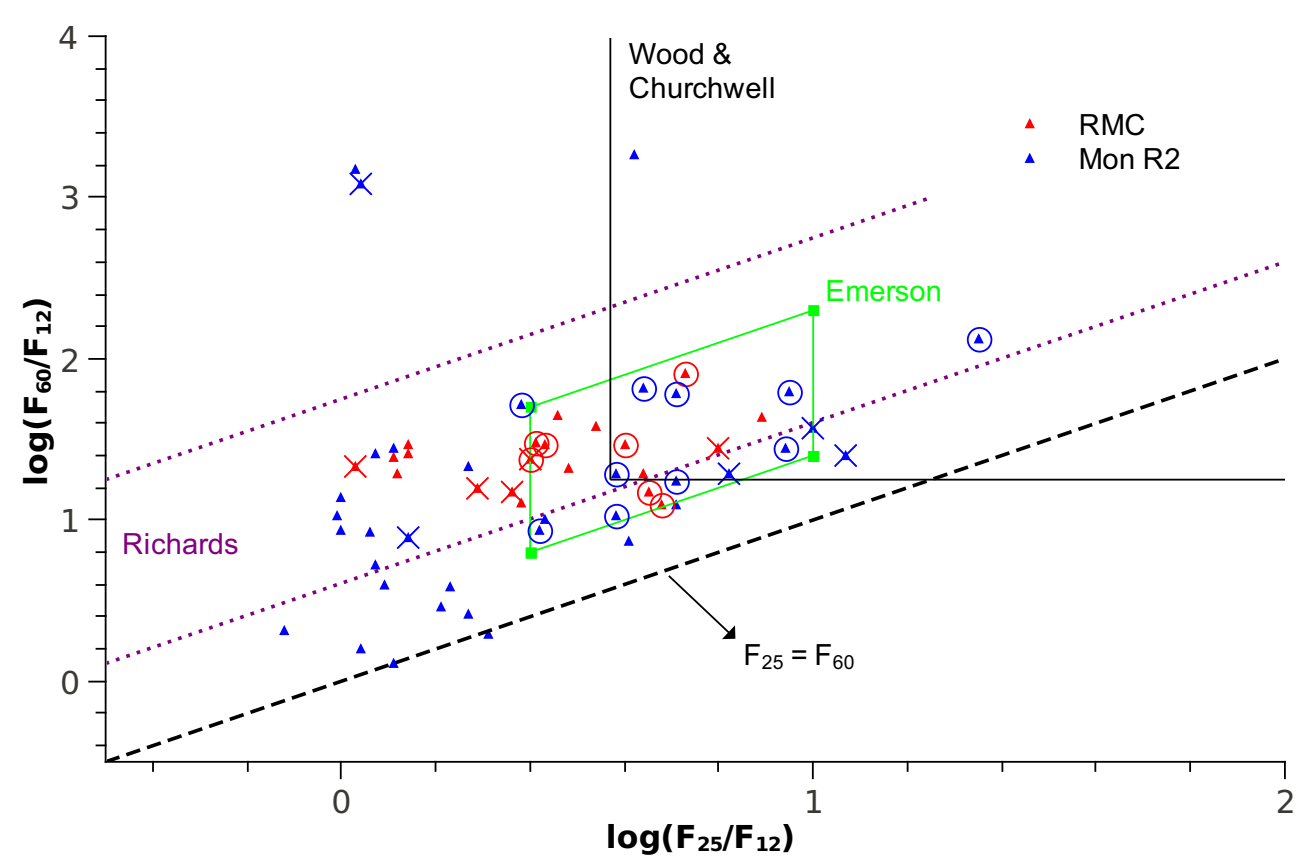

Figura 5.10: Distribuição das fontes IRAS associadas RMC (triângulos vermelhos) e Mon R2 (triângulos azuis) no diagrama de cores [60-12] x [25-12], que indica as regiões sugeridas por Wood e Churchwell (1989), para separar as candidatas a regiões UC HII (acima das linhas cheias, em preto), e por Richards et al. (1987) para selecionar clumps densos (entre as linhas pontilhadas, em roxo). A linha tracejada, em preto, mostra $\mathrm{F}_{25}=\mathrm{F}_{60}$. As fontes de raios-X são indicada por "X". Círculos indicam a coincidência com masers, os quais ocupam preferencialmente a região central, definida por Emerson (1987) (linhas cheias verdes).

Codella e Palla (1995) utilizaram os critérios de cores IRAS ilustrados na Figura 5.10 para selecionar candidatos a masers de $\mathrm{H}_{2} \mathrm{O}$ e estudar a natureza de protoestrelas massivas. 
Eles verificaram uma taxa de detecção muito baixa (11 masers em 140 candidatas) e encontraram uma forte dependência da densidade de fluxo em $60 \mu \mathrm{m}$. As fontes mais brilhantes $\left(\mathrm{F}_{60}>100 \mathrm{Jy}\right)$ são as mais prováveis de apresentar emissão maser e que deve ser pouco provável que fontes com $[25-12]<0.57$ estejam associadas a formação estelar de alta massa. No caso de nossa amostra, a maioria das fontes $\operatorname{IRAS}$ apresenta as cores típicas de objetos selecionados por Codella e Palla (1995), que aparecem entre as linhas pontilhadas. Todos os masers encontram-se no locus sugerido por Codella et al. (1995), exceto IRAS 06084-0611, uma fonte muito brilhante, que apresenta cores IRAS superiores às estudadas por aqueles autores. Em RMC, todos os 7 masers associados a fontes IRAS coincidem com a região esperada para os cores moleculares (Richards et al., 1987), mas apenas duas se encontram na região das UC HII (Wood e Churchwell, 1989), não sendo consideradas fontes brilhantes em $60 \mu \mathrm{m}$. Por outro lado, em Mon R2 dos 10 masers associados com fontes $I R A S, 7$ aparecem na região das $U C H I I$, sendo apenas 3 delas consideradas brilhantes $\left(\mathrm{F}_{60}>100 \mathrm{Jy}\right)$. Ou seja, cerca de 8/17 de fontes $I R A S$ de nossa amostra não são consideradas candidatas a apresentarem masers associados com UC HII.

De fato, Codella e Felli (1995) argumentam que uma boa fração dos masers de $\mathrm{H}_{2} \mathrm{O}$ não está associada a emissão difusa de regiões HII, sugerindo que esta subclasse de objetos representaria uma fase evolutiva anterior, na qual a região HII ainda não se formou. No que se refere ao status evolutivo de nossa amostra, é interessante notar a falta de correlação entre as fontes maser e as fontes de raios-X, sugerindo que as primeiras indicam candidatas para a fase protoestelar, enquanto que as últimas representam a fase $P M S$.

\subsection{Aglomerados Jovens}

As Figuras 4.8 e 4.9 mostram as coincidências na posição das fontes listadas nas Tabelas 4.2 e 4.3 para RMC e Mon R2 respectivamente. É interessante notar que a distribuição dos objetos selecionados em RMC segue uma linha aproximadamente paralela ao plano galáctico, que coincide com as regiões mais densas (vide Figura 2.5). Em Mon R2 os objetos acompanham a estrutura filamentária da nuvem, a qual tem uma inclinação de aproximadamente $-60^{\circ}$ com relação ao plano, que pode ser considerada uma relevante diferença entre as duas regiões estudadas e pode estar ligada ao cenário de formação estelar de ambas, como discutiremos na Seção 5.6. 
Somente no caso de RMC foi possível correlacionar as fontes com clumps e aglomerados jovens. Como pode ser visto na Figura 4.8, a maior concentração de objetos ocorre para a sub-região C, que contém 4 clumps, 5 clusters, 2 masers e 4 fontes-X.

Os aglomerados jovens também foram avaliados por meio das composições de imagens 2MASS. Estudos nessa linha podem contribuir para a compreensão de formação estelar dentro de nuvens moleculares, indicando pistas sobre a formação de estrelas isoladas ou em aglomerados, sobre mecanismos de formação desencadeada ou espontânea e, até mesmo, sob quais ambientes estrelas de massas diferentes se formam. Nesse contexto, Phelps e Lada (1997) realizaram uma inspeção visual, destacando 7 aglomerados jovens embebidos, localizados em RMC e associados à fontes IRAS.

Nesse estudo, os autores localizaram os algomerados sobre o mapa de ${ }^{13} \mathrm{CO}$ apresentado por Blitz e Stark (1986), como mostra a Figura 5.11. Quatro desses aglomerados foram estudados por nós neste tabalho e são destacados em quadrados: vermelho (sub-região A), verde (sub-região B), magenta (sub-região C) e azul (sub-região D). A posição dos aglomerados parece se relacionar com a distribuição de ${ }^{13} \mathrm{CO}$.

As Figuras 4.22, 4.23, 4.24 e 4.25 nos remete a uma análise mais aprofundada dessas sub-regiões presentes em Mon R2, uma vez que uma composição RGB das bandas JHK fornece informações a respeito dos excessos no infravermelho característicos de aglomerados jovens, podendo revelar informações sobre a população estelar embebida em clumps densos aos quais encontram-se associados. Como exemplo, podemos citar Higuchi et al. (2009), que utilizaram dados 2MASS para obter informações acerca de diversas regiões, incluindo o core central de Mon R2 (Figura 5.12), por eles classificado como UC HII.

A estrutura brilhante e avermelhada, no centro da imagem, revela excesso na banda K. As nebulosas de reflexão se destacam pela coloração azulada, pois são iluminadas por estrelas B.

Também interessados em investigar aglomerados em Mon R2 utilizando observações no infravermelho, Maaskant et al. (2011) realizaram um estudo para examinar o conteúdo estelar da região GGD 12-15, Dados do ESO New Technology Telescope (NTT/SOFI), 2MASS e Spitzer/IRAC permitiram determinar o tipo espectral da maior parte das estrelas luminosas do aglomerado. Muitos objetos apresentam excesso no infravermelho, sendo 7 deles classificados como Classe 0/I e 5 objetos como Classe II. Uma imagem $\operatorname{IRAC}(4.5 \mu \mathrm{m})$ 


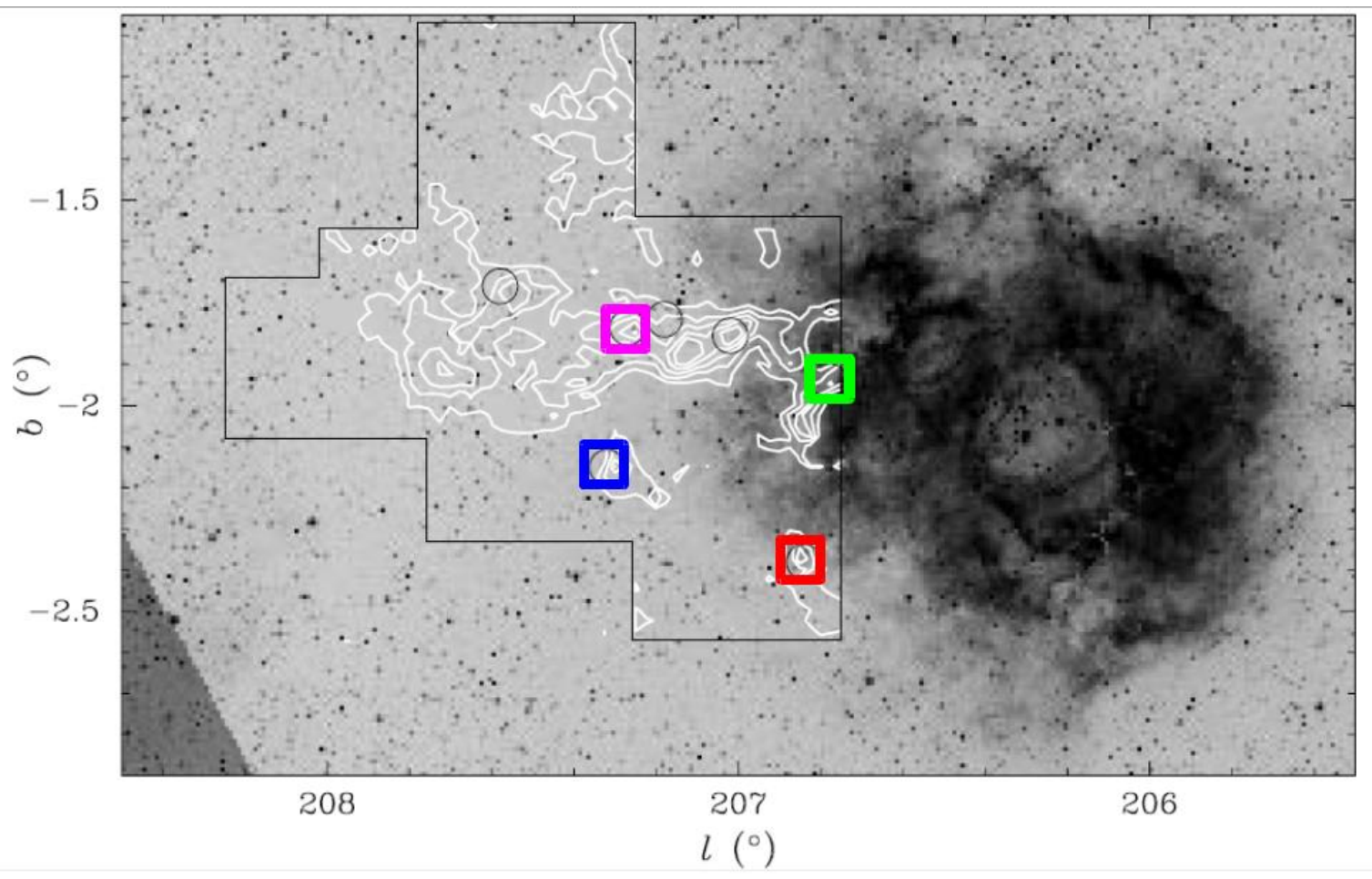

Figura 5.11: Imagem $D S S$ de RMC. Os círculos pretos indicam os algomerados embebidos. Os quadrados (que também contêm círculos pretos) indicam os aglomerados apresentados na Tabela 2.2 (PL01-07). O mapa de contornos em ${ }^{13} \mathrm{CO}$ (em branco) foram obtidos de Blitz e Stark (1986). Figura adaptada de Phelps e Lada (1997).

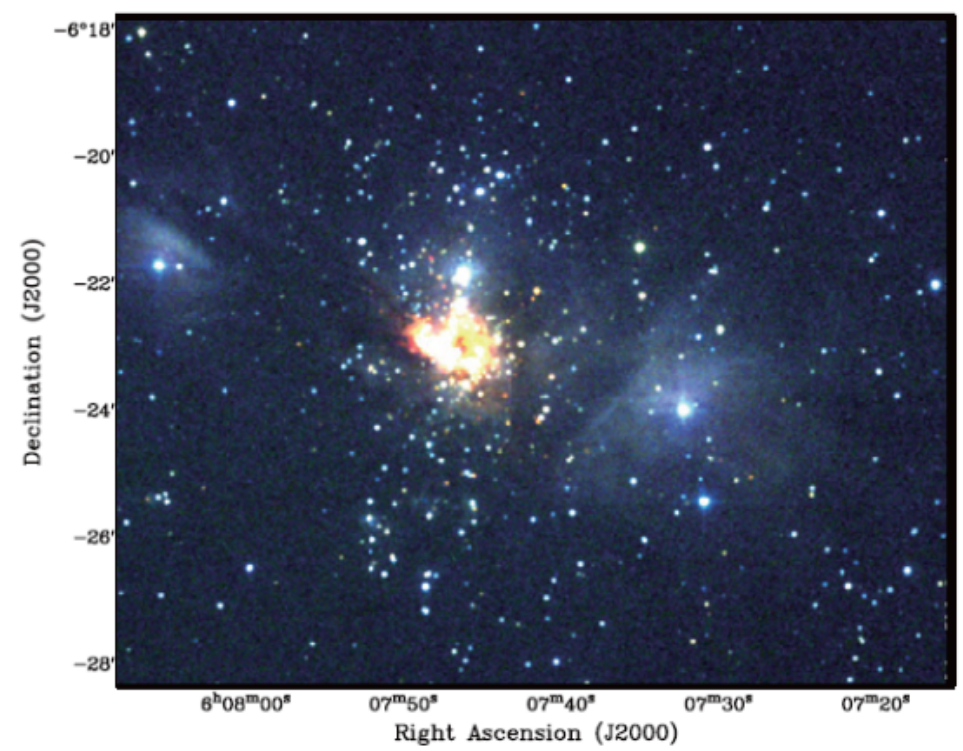

Figura 5.12: Composição RGB das bandas JHK do 2MASS do core central de Mon R2. Figura adaptada de Higuchi et al. (2009). 
mostra a região em questão, ressaltando as nebulosidades do aglomerado, que se destaca na parte central da Figura 5.13.

A Figura 5.14 mostra a mesma região, desta vez incluindo a posição de objetos evoluídos, estudados anteriormente por Lada (1987). A distribuição espacial desses objetos indica que os Classe 0/I estão mais centralmente localizados se comparados com os objetos Classe II, que são mais evoluídos.

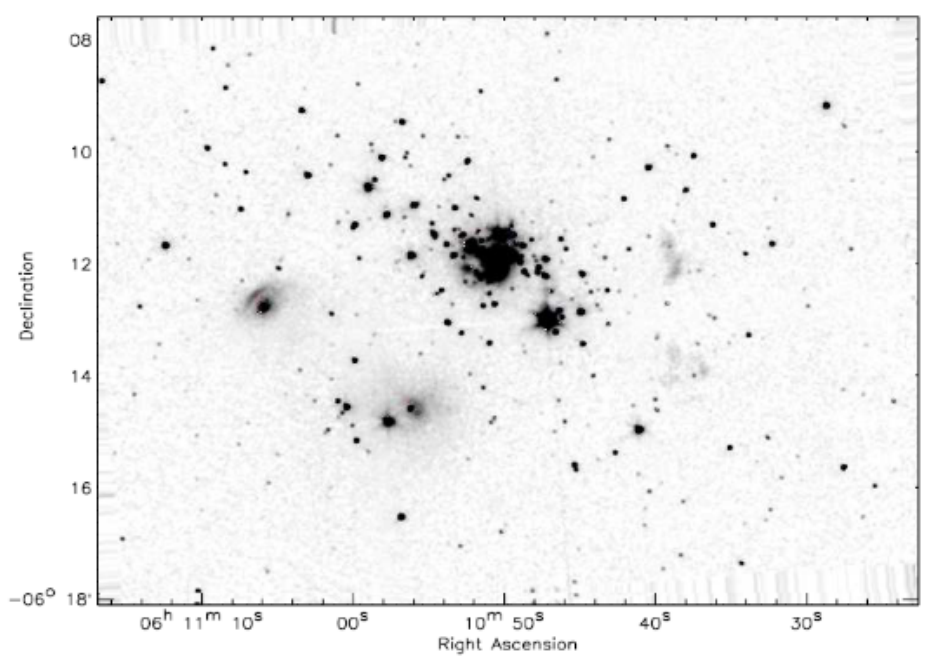

Figura 5.13: Imagem, no infravermelho próximo, da região GGD 12-15, localizada em Mon R2. Figura adaptada de Maaskant et al. (2011).

A presença de uma região $U C H I I$ e objetos altamente avermelhados nessa região sugere uma idade bastante jovem para o aglomerado $(<1 \mathrm{Myr})$. No caso da estrela T Tauri Bretz 4, citada anteriormente, é um objeto interessante, também identificado como HR 29 (Herbig e Kameswara Rao, 1972). Um estudo óptico realizado por Carballo e Eiroa (1992) mostrou que esse objeto está associado com GGD 17 e a nebulosidade próxima à estrela mostra a morfologia típica de luz espalhada. A Figura 5.15 mostra o sistema Bretz 4/GGD 17 que, na imagem [SII], apresenta um jato brilhante e altamente colimado.

Com efeito, a estrutura filamentária é observada na Figura 4.20. A mesma estrutura pode ser observada, com menos destaque, na Figura 4.24 e o excesso na banda K aparece como uma componente avermelhada ao lado da estrela Bretz 4, mostrando conexão morfológica com a poeira presente em GGD 17. 


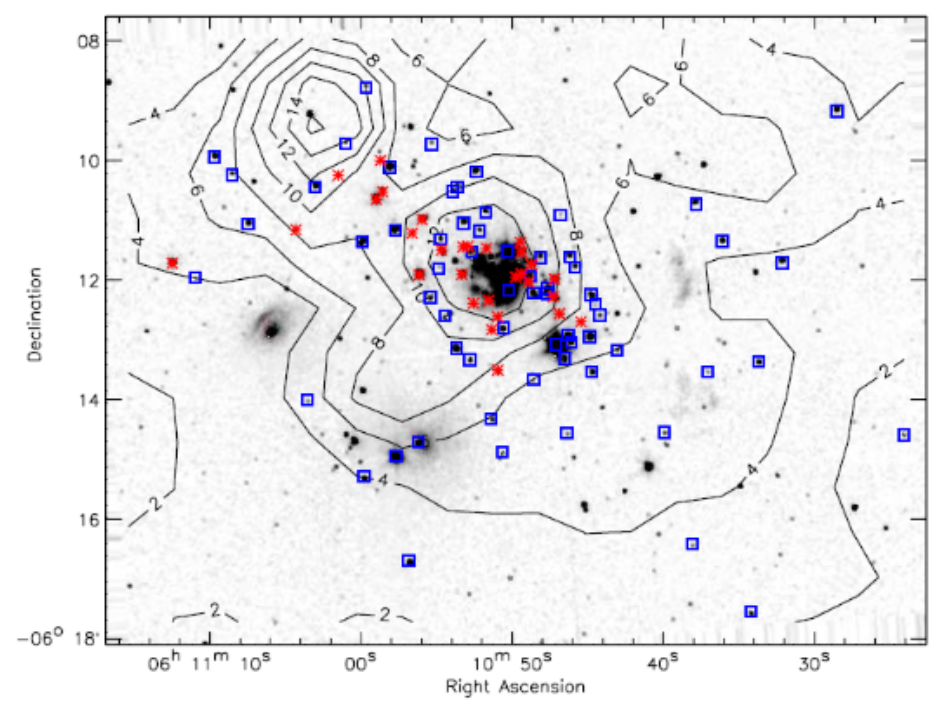

Figura 5.14: Posição dos objetos Classe 0/I (asteriscos vermelhos) e Classe II (quadrados azuis), sobrepostos em mapa IRAC na banda $4.5 \mu \mathrm{m}$. Contornos representam níveis de $\mathrm{A}_{V}$. Figura de Maaskant et al. (2011).

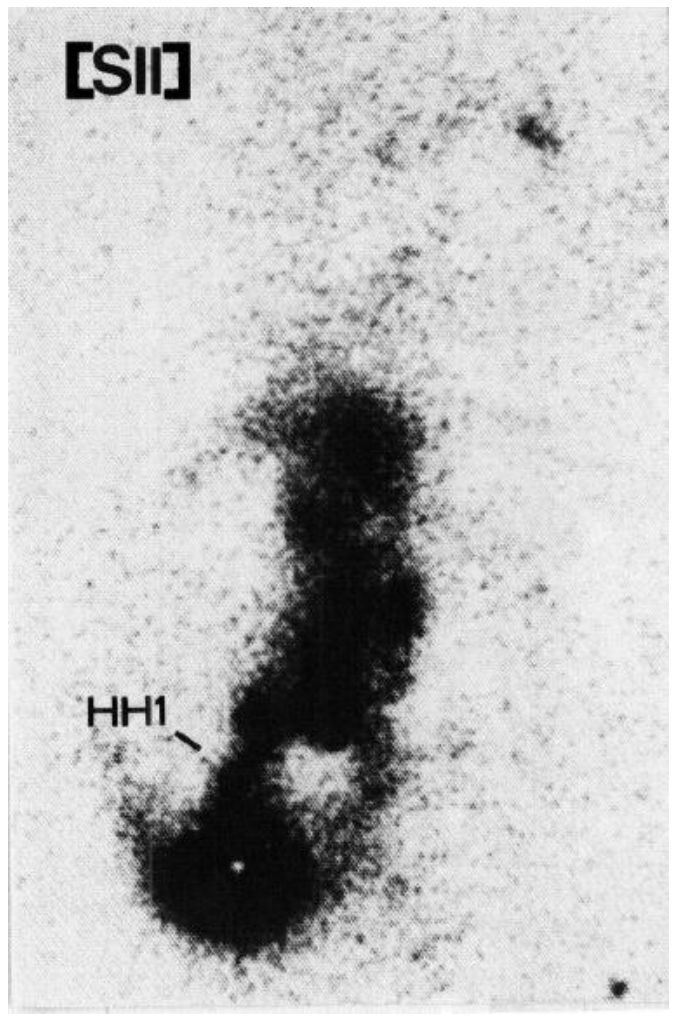

Figura 5.15: Imagem [SII] do sistema Bretz 4/GGD 17. O campo corresponde a $0^{\prime} .8 \times 1^{\prime} .2$. Figura de Carballo e Eiroa (1992). 


\subsection{O cenário de formação estelar no Complexo de Monoceros}

Para concluir nossa análise, confrontamos aqui as características levantadas para os objetos estudados, em termos de distribuição espacial, com a hipótese de formação estelar provocada pela passagem de HVCs atravessando o plano galáctico, proposta originalmente por Mirabel e Franco (1981) e posteriormente avaliada em mais detalhes por Lépine e Duvert (1994) e Hetem e Lépine (1993). De acordo com o mencionado no Capítulo 1, simulações numéricas realizadas nesses trabalhos tiveram sucesso em reproduzir a distribuição de gás ao longo da passagem de uma nuvem hipoteticamente esférica em queda inclinada. A Figura 1.6 mostra a simulação da passagem de uma $H V C$ que arrastou o gás do plano galáctico para baixas latitudes, possivelmente produzindo a distribuição de nuvens que vemos hoje no complexo de Orion-Monoceros. As limitações de tal modelo (distribuição uniforme de gás, tanto no plano Galáctico como na $H V C$ ) e a visão projetada, não permitiram simular a estrutura de Mon R2. No entanto, as características filamentárias desta nuvem, com semelhante inclinação com toda a estrutura que poderia ter sido gerada pela passagem da $H V C$, sugere a possível origem comum para todo o conjunto de nuvens, porém com condições iniciais mais complexas do que aquelas supostas no trabalho de Hetem (1996), por exemplo.

Apesar de se encontrar fora da região onde provavelmente ocorreu o arrasto de material, RMC também poderia ter sofrido os efeitos da passagem da $H V C$, porém com uma compressão no sentido do plano galáctico, sem provocar substancial deslocamento e/ou mistura do gás das nuvens em choque. Essa diferença no sentido de compressão do gás poderia ser responsável pelas diferenças nas distribuições de objetos jovens, quando se compara RMC com Mon R2. Apesar das coincidências encontradas em ambas as nuvens, tanto para a distribuição de objetos jovens quanto para distribuição de gás e poeira, as quais corroboram a proposta das $H V C s$, tal hipótese não é exclusiva para explicar o cenário de formação estelar no Complexo Orion-Monoceros. Como discutiremos no Cap. 6, outras propostas mais recentes, relacionadas com a dinâmica da Galáxia, também devem ser levadas em conta.

A menor quantidade de masers encontrados em RMC também pode revelar uma menor atividade de formação estelar.

Em RMC, as duas fontes mais luminosas em $160 \mu \mathrm{m}$ detectadas pelo satélite Herschel e 
apresentadas por Motte et al. (2010) (vide Figura 1.4) são AFGL 961 e IRAS 06308+0402, classificadas como cores protoestelares densos de alta massa, brilhantes no infravermelho. Essas fontes possuem massa da ordem de $22 \mathrm{M}_{\odot}$, temperatura da ordem de $30 \mathrm{~K}$, luminosidade que varia de 1 a 4 x $10^{3} \mathrm{~L}_{\odot}$ e são consideradas precursoras de estrelas OB (Cox et al., 1990). Outras quatro fontes são igualmente massivas, porém menos brilhantes (15 a 75 $\left.\mathrm{L}_{\odot}\right)$ e mais frias $(15 \mathrm{~K})$. Embora estas sejam consideradas boas candidatas para hospedar protoestrelas massivas em sua fase inicial, são necessárias outras observações que indiquem jatos, cores quentes, e/ou emissão maser.

Uma fonte-X peculiar também estudada com interesse em RMC é IRAS 06306+0437, identificada por Wang et al. (2009) como RMCX \#89. Trata-se de uma protoestrela massiva, localizada no clump \#2 de Phelps e Lada (1997) (PL02, vide Tabela 2.2), que exibe um excesso incomum na banda $\mathrm{K}$ do infravermelho próximo, apresentando forte avermelhamento (da ordem de $\mathrm{A}_{V} \sim 30$ ). Wang et al. (2009) destacam que IRAS 06306+0437 é uma conhecida fonte maser de água, que está em um ambiente empoeirado. Outra candidata a protoestrela, RMCX \#72, está próxima de RMCX \#89 em uma condensação secundária de poeira.

Algumas fontes IRAS altamente luminosas podem ser destacadas em RMC. IRAS 06319+0415, por exemplo, possui $\mathrm{L}_{I R} \sim 11000 \mathrm{~L}_{\odot}$, localizada em um dos maiores clusters da região, PL06. Esta fonte infravermelha não é tão intensa quanto IRAS 06053-0622 (presente em Mon R2) mas ainda assim a mais luminosa das fontes IRAS em RMC. A fonte $\operatorname{IRAS} 06308+0402$ tem $\mathrm{L}_{I R} \sim 2500 \mathrm{~L}_{\odot}$, destacando-se na sub-região A.

Diversas fontes IRAS detectadas em Mon $\mathrm{R} 2$ apresentam $\mathrm{L}_{I R}$ na faixa de unidades a centenas de $\mathrm{L}_{\odot}$, porém dois objetos destacam-se pela alta luminosidade infravermelha: $\operatorname{IRAS} 06053-0622\left(\mathrm{~L}_{I R} \sim 26000 \mathrm{~L}_{\odot}\right)$ e $I R A S 06084-0611\left(\mathrm{~L}_{I R} \sim 5600 \mathrm{~L}_{\odot}\right)$. A primeira fonte faz parte do core central de Mon R2, associada a um aglomerado com $\sim 300$ estrelas. É uma UC HII que possui uma fonte de maser de água bastante intensa ( $\left.\mathrm{F}_{\text {pico }} \sim 100 \mathrm{Jy}\right)$ - o maser mais intenso de Mon R2. A segunda fonte citada, IRAS 06084-0611, está localizada na rica região GGD 12-15 (vide Fig. 5.13). A fonte IRAS 06103-0612 também se sobressai por possuir $\mathrm{F}_{\text {pico }} \sim 46 \mathrm{Jy}$; esta fonte tem destaque na região GGD 16-17.

A fonte IRAS 06306+0437 merece atenção especial por ser a fonte maser mais intensa de RMC, apresentando $\mathrm{F}_{\text {pico }} \sim 21 \mathrm{Jy}$. 
Os algomerados jovens estudados por Carpenter (2000) na banda do infravermelho próximo e identificados por meio da densidade superficial de estrelas apontam uma preferência na distribuição de objetos em Mon R2. As quatro regiões destacadas pelos autores se mostram alinhadas com a estrutura filamentária de gás molecular, que correlaciona-se também com a distribuição de poeira na nuvem (vide Figura 4.7). 
Capítulo 6

\section{Considerações Finais}

\subsection{Conclusões}

A metodologia utilizada para a comparação das duas nuvens de interesse envolveu estudo em diferentes bandas espectrais. O levantamento de objetos nessas regiões e a análise de mapas nos permitiu verificar algumas semelhanças entre RMC e Mon R2, mas também revelou diferenças interessantes.

Quando comparamos a distribuição de gás $(\mathrm{CO})$ e poeira $\left(\mathrm{A}_{V}\right)$, ambas nuvens apresentam boa correlação entre os picos de intensidade. Entretanto, RMC possui uma distribuição de poeira mais homogênea, se comparada com Mon R2, que apresenta estruturas mais filamentárias. Notamos também que a extinção e os picos de emissão de CO são mais elevados no core de Mon R2, em níveis comparáveis com os de Orion, superiores aos encontrados para as demais sub-regiões aqui estudadas. Embora a emissão em $100 \mu \mathrm{m}$ tenha boa correlação com a distribuição de gás molecular, os mapas de CO apresentam baixa resolução espacial; assim, optamos por explorar a distribuição da razão $\mathrm{F}_{60} / \mathrm{F}_{100}$ para distinguir emissão estelar da interestelar. Ao confrontar a razão de fluxos com a extinção visual, observamos que a emissão estelar nem sempre coincide com as áreas de maior $\mathrm{A}_{V}$ e, além disso, ocorre emissão interestelar em locais de baixa extinção. Uma forma de verificar tais correspondências foi comparar a extinção com a razão $\mathrm{F}_{60} / \mathrm{F}_{100}$ obtida para os masers das regiões, porém não encontramos uma tendência em particular. Já se compararmos a intensidade da emissão maser com a razão $\mathrm{F}_{60} / \mathrm{F}_{100}$, encontramos uma correlação aproximada, na qual masers melhor detectados e mais intensos tendem a apresentar maiores valores para a razão de fluxos $\mathrm{F}_{60} / \mathrm{F}_{100}$. Ou seja, quanto maior a emissão estelar, maior a emissão maser. 
Ao estudar os clumps presentes em RMC, a riqueza de informações permitiu comparar alguns parâmetros físicos como extinção visual, dimensão do fragmento e tempe- ratura. De forma geral, clumps maiores apresentam maior extinção e menor temperatura, enquanto clumps mais densos possuem menor temperatura devido à alta concentração de poeira fria. Clumps também foram observados em Mon R2, mas a falta de informações não permitiram um estudo comparativo.

Em relação à população estelar, a maior diferença entre as nuvens é a presença de estrelas massivas do tipo $\mathrm{O}$ em RMC, distribuídas uniformemente, mas ainda assim concentradas em um aglomerado aberto, ao passo que Mon R2 apresenta estrelas B distribuídas ao longo de um filamento de gás molecular $(\mathrm{CO})$. Rosette apresenta uma grande nebulosa de destaque, enquanto Mon R2 abriga diversas pequenas nebulosas de reflexão associadas a regiões com maior quantidade de poeira. Objetos de diferentes catálogos parecem ser espacialmente coincidentes, embora Mon R2 apresente um número ligeiramente maior de fontes coincidentes em relação à RMC. Em termos de densidade superficial de objetos, notamos que Mon R2 tem uma população jovem mais rica do que em Rosette, porém não necessariamente mais jovem. Ao confrontarmos características de fontes-X detectadas em ambas as nuvens, encontramos para Mon R2 uma função de luminosidade X próxima à de Cha 1, que possui objetos com idades da ordem de $10^{7}$ anos; por outro lado, no caso de RMC a distribuição é superior à de $\rho$ Oph, que possui membros com $\sim 10^{6}$ anos. Ou seja, as fontes-X de RMC tendem a ser mais jovens.

O estudo de estágios evolutivos de fontes IRAS envolveu a identificação de possíveis protoestrelas através da comparação entre as cores $\operatorname{IRAS}\left(\mathrm{F}_{25} / \mathrm{F}_{12}\right.$ e $\left.\mathrm{F}_{60} / \mathrm{F}_{12}\right)$ e emissão maser de $\mathrm{H}_{2} \mathrm{O}$, selecionando assim candidatas a $U C$ HII. Os masers por nós estudados apresentam características típicas de protoestrelas massivas. No caso de nossa amostra, não há uma correlação clara entre fontes maser e fontes-X, sugerindo que emissão maser pode indicar fase protoestelar, enquanto fontes-X representam a fase $P M S$.

Os aglomerados jovens selecionados na nuvem Rosette distribuem-se paralelamente ao plano da Galáxia, que coincide com regiões mais densas. Em Mon R2 esses aglomerados localizam-se em uma estrutura filamentária inclinada em $-60^{\circ}$ com relação ao plano galáctico. Essa diferença entre as duas regiões se mostrou relevante e pode estar relacionada com o cenário de formação estelar em cada uma delas. Em RMC, os aglomerados 
se encontraram bem relacionados com a distribuição molecular observada no mapa de ${ }^{13} \mathrm{CO}$ e mostraram ter excessos no infravermelho típicos de população jovem embebida. Em Mon R2, o aglomerado GGD 12-15 mereceu destaque por apresentar objetos Classe 0/I centralmente localizados e boa correspondência com o pico de extinção visual.

Os algomerados jovens foram avaliados por meio de imagens $2 M A S S$, o que não permitiu a extração de uma grande quantidade de informações e precisão na análise. Alguns resultados obtidos por outros autores foram apresentados com a finalidade de mostrar a necessidade de utilizar dados de melhor qualidade, como Spitzer/IRAC por exemplo.

Concluindo a análise, confrontamos características dos objetos estudados com a hipótese de formação estelar provocada pela passagem de $H V C$ pelo o plano Galáctico. Verificamos que nossos resultados corroboram as simulações numéricas que reproduzem a distribuição de gás observada. Limitações nesse modelo, entretanto, não permitiram simular toda a estrutura, porém a hipótese sugere que todo o conjunto de nuvens possa ter origem comum, ainda que em condições mais complexas do que as apresentadas nas simulações. RMC pode ter sofrido com a passagem de $H V C$, mas a compressão não teria provocado deslocamento da nuvem para fora do plano, mas teria ocorrido no sentido do plano da Galáxia. Mon R2 encontra-se na região onde ocorreu o arrasto de material, o que sugere que a nuvem foi levada para fora do plano galáctico e o gás teria sido comprimido em um sentido inclinado. Tal distinção poderia ser a causa das diferenças nas distribuições dos YSOs ao compararmos RMC com Mon R2.

\subsection{Perspectivas}

As perspectivas de continuação desse trabalho podem se basear em:

- aplicar o estudo comparativo para outras nuvens moleculares, como CMa R1, que também se encontra no Complexo de Monoceros;

- estudar a função de luminosidade-X com dados Chandra (Getman et al., 2005; Kohno et al., 2002; Wang et al., 2009) e XMM (Jeffries et al., 2009; Guedel et al., 2007).

- utilizar os métodos aqui adotados para confrontar com outros cenários de formação estelar, como por exemplo modelos de formação estelar sequencial (Blaauw, 1991) e 
o modelo de Gould Belt (Olano e Poeppel, 1987), além da interpretação de braços espirais (Sartori et al., 2003);

- dar continuidade às observações realizadas no ROI, para a possível detecção de mais masers de $\mathrm{H}_{2} \mathrm{O}$, e posteriormente reduzir e analisar os dados obtidos, de modo à apresentá-los de forma sistemática e conclusiva (Monfredini, 2009; Almeida, 2006);

- levantar características de YSOs para correlacioná-las com fontes observadas em diferentes comprimentos de onda, selecionando outros alvos para possível detecção de maser em rádiofrequencias. 


\section{Referências Bibliográficas}

Alcalá J. M., Krautter J., Schmitt J. H. M. M., Covino E., Wichmann R., Mundt R., A study of the Chamaeleon star forming region from the ROSAT all-sky survey. I. X-ray observations and optical identifications., A\&AS, 1995, vol. 114, p. 109

Almeida U. B., Observações em Radiofrequências do Complexo de Regiões HII Compactas em RCW 95, São Paulo: Universidade de São Paulo - USP, 2006, Dissertação de Mestrado

André P., Belloche A., Motte F., Peretto N., The initial conditions of star formation in the Ophiuchus main cloud: Kinematics of the protocluster condensations, A\&A, 2007, vol. 472 , p. 519

André P., Montmerle T., From T Tauri stars to protostars: Circumstellar material and young stellar objects in the rho Ophiuchi cloud, ApJ, 1994, vol. 420, p. 837

André P., Ward-Thompson D., Barsony M., Submillimeter continuum observations of Rho Ophiuchi A - The candidate protostar VLA 1623 and prestellar clumps, ApJ, 1993, vol. 406 , p. 122

André P., Ward-Thompson D., Barsony M., From Prestellar Cores to Protostars: the Initial Conditions of Star Formation, Protostars and Planets IV, 2000, p. 59

Beckwith S. V. W., Sargent A. I., Chini R. S., Guesten R., A survey for circumstellar disks around young stellar objects, AJ, 1990, vol. 99, p. 924 
Beichman C. A., Myers P. C., Emerson J. P., Harris S., Mathieu R., Benson P. J., Jennings R. E., Candidate solar-type protostars in nearby molecular cloud cores, ApJ, 1986, vol. 307 , p. 337

Berghöefer T. W., Schmitt J. H. M. M., Danner R., Cassinelli J. P., X-ray properties of bright OB-type stars detected in the ROSAT all-sky survey., A\&A, 1997, vol. 322, p. 167

Bergin E. A., van Dishoeck E. F., Water in star- and planet-forming regions, Royal Society of London Philosophical Transactions Series A, 2012, vol. 370, p. 2778

Blaauw A., OB Associations and the Fossil Record of Star Formation, 1991, p. 125

Blitz L., Stark A. A., Detection of clump and interclump gas in the Rosette Molecular Cloud Complex, ApJ, 1986, vol. 300, p. L89

Blitz L., Thaddeus P., Giant molecular complexes and OB associations. I - The Rosette molecular complex, ApJ, 1980, vol. 241, p. 676

Bloemen J. B. G. M., Deul E. R., Thaddeus P., Decomposition of the FIR Milky Way observed by IRAS, A\&A, 1990, vol. 233, p. 437

Bonnell I. A., Vine S. G., Bate M. R., Massive star formation: nurture, not nature, MNRAS, 2004, vol. 349, p. 735

Breen S. L., Caswell J. L., Ellingsen S. P., Phillips C. J., Water masers accompanying OH and methanol masers in star formation regions, MNRAS, 2010, vol. 406, p. 1487

Breen S. L., Ellingsen S. P., Johnston-Hollitt M., A search for 22-GHz water masers within the giant molecular cloud associated with RCW 106, MNRAS, 2007, vol. 377, p. 491

Cambrésy L., Beichman C. A., Jarrett T. H., Cutri R. M., Extinction with 2MASS: Star Counts and Reddening toward the North America and Pelican Nebulae, AJ, 2002, vol. 123 , p. 2559

Carballo R., Eiroa C., A Herbig-Haro flow associated with the T Tauri star Bretz 4 in GGD 17, A\&A, 1992, vol. 262, p. 295 
Carpenter J. M., 2MASS Observations of the Perseus, Orion A, Orion B, and Monoceros R2 Molecular Clouds, AJ, 2000, vol. 120, p. 3139

Carpenter J. M., Hodapp K. W., , 2008 The Monoceros R2 Molecular Cloud. p. 899

Casoli F., Combes F., Dupraz C., Gerin M., Boulanger F., (C-13)O and (C-12)O observations of cold IRAS unidentified point sources in the Galaxy, A\&A, 1986, vol. 169, p. 281

Celnik W. E., The Rosette nebula. I - an absolutely calibrated photoelectric H-alpha surface photometry, A\&AS, 1983, vol. 53, p. 403

Cheung A. C., Rank D. M., Townes C. H., Detection of Water in Interstellar Regions by its Microwave Radiation, Nature, 1969, vol. 221, p. 626

Codella C., Felli M., H_2_O masers without associated diffuse HII regions: an earlier evolutionary phase?, A\&A, 1995, vol. 302, p. 521

Codella C., Palla F., The nature of massive protostellar candidates. I. A search for water masers towards color-selected IRAS sources., A\&A, 1995, vol. 302, p. 528

Codella C., Palumbo G. G. C., Pareschi G., Scappini F., Caselli P., Attolini M. R., IRASselected Galactic star-forming regions - II. Water maser detections in the extended sample, MNRAS, 1995, vol. 276, p. 57

Cox P., Deharveng L., Leene A., IRAS observations of the Rosette nebula complex, A\&A, 1990, vol. 230, p. 181

Dame T. M., Hartmann D., Thaddeus P., The Milky Way in Molecular Clouds: A New Complete CO Survey, ApJ, 2001, vol. 547, p. 792

De Marco O., O’Dell C. R., Gelfond P., Rubin R. H., Glover S. C. O., Cloud Fragmentation and Proplyd-like Features in H II Regions Imaged by the Hubble Space Telescope, AJ, 2006, vol. 131, p. 2580

Dobashi K., Uehara H., Kandori R., Sakurai T., Kaiden M., Umemoto T., Sato F., Atlas and Catalog of Dark Clouds, VizieR Online Data Catalog, 2005, vol. 7244, p. 0 
Dolan C. J., Mathieu R. D., A Photometric Study of the Young Stellar Population throughout the $\lambda$ Orionis Star-Forming Region, AJ, 2002, vol. 123, p. 387

Dorschner J., Gürtler J., Untersuchungen über Reflexionsnebel am Palomar Sky Survey I. Verzeichnis von Reflexionsnebeln, Astronomische Nachrichten, 1963, vol. 287, p. 257

Elitzur M., Astronomical Masers, ARA\&A, 1992, vol. 30, p. 75

Emerson J. P., IRAS and star formation in dark clouds. In Star Forming Regions, vol. 115 of IAU Symposium, 1987, p. 19

Feigelson E. D., Decampli W. M., Observations of X-ray emission from T Tauri stars, ApJ, 1981, vol. 243, p. L89

Feigelson E. D., Montmerle T., High-Energy Processes in Young Stellar Objects, ARA\&A, 1999, vol. 37, p. 363

Franco J., Tenorio-Tagle G., Bodenheimer P., Rozyczka M., Mirabel I. F., On the origin of the Orion and Monoceros molecular cloud complexes, ApJ, 1988, vol. 333, p. 826

Getman K. V., Feigelson E. D., Grosso N., McCaughrean M. J., Micela G., Broos P., Garmire G., Townsley L., Membership of the Orion Nebula Population from the Chandra Orion Ultradeep Project, ApJS, 2005, vol. 160, p. 353

Gregorio-Hetem J., , 2003 Tese de Livre Docência

Gregorio-Hetem J., Montmerle T., Casanova S., Feigelson E. D., X-rays and star formation: ROSAT observations of the Monoceros and Rosette molecular clouds, A\&A, 1998, vol. 331, p. 193

Gregorio-Hetem J., Montmerle T., Rodrigues C. V., Marciotto E., Preibisch T., Zinnecker H., Star formation history of Canis Major R1. I. Wide-Field X-ray study of the young stellar population, A\&A, 2009, vol. 506, p. 711

Guedel M., Briggs K. R., Arzner K., Audard M., Bouvier J., Feigelson E. D., Franciosini E., Glauser A., Grosso N., Telleschi A., XMM-Newton Extended Survey of Taurus (Guedel+, 2007), VizieR Online Data Catalog, 2007, vol. 346, p. 80353 
Gyulbudaghian A. L., Glushkov Y. I., Denisyuk E. K., New Herbig-Haro objects, ApJ, 1978, vol. 224, p. L137

Herbig G. H., Kameswara Rao N., Second Catalog of Emission-Line Stars of the Orion Population, ApJ, 1972, vol. 174, p. 401

Herbst W., Racine R., R-associations. V. Monoceros R2., AJ, 1976, vol. 81, p. 840

Hetem A., Estudo da estrutura de nuvens moleculares, São Paulo: Universidade de São Paulo - USP, 1996, Tese de Doutorado

Hetem Jr. A., Shocks of High Velocity Clouds and the Galactic Disk: 3D Numerical Simulations, Ap\&SS, 1995, vol. 233, p. 181

Hetem Jr. A., Lépine J. R. D., Numerical Models for Shocks and Wave Propagation in Dense Cloud Cores, Rev. Mexicana Astron. Astrofis., 1993, vol. 26, p. 96

Heyer M. H., Williams J. P., Brunt C. M., Turbulent Gas Flows in the Rosette and G216-2.5 Molecular Clouds: Assessing Turbulent Fragmentation Descriptions of Star Formation, ApJ, 2006, vol. 643, p. 956

Higuchi A. E., Kurono Y., Saito M., Kawabe R., A Mapping Survey of Dense Clumps Associated with Embedded Clusters: Evolutionary Stages of Cluster-forming Clumps, ApJ, 2009, vol. 705, p. 468

Jeffries R. D., Naylor T., Walter F. M., Pozzo M. P., Devey C. R., The stellar association around Gamma Velorum and its relationship with Vela OB2, MNRAS, 2009, vol. 393, p. 538

Kohno M., Koyama K., Hamaguchi K., Chandra Observations of High-Mass Young Stellar Objects in the Monoceros R2 Molecular Cloud, ApJ, 2002, vol. 567, p. 423

Kutner M. L., Tucker K. D., CO, CS, and HCN in a clustering of reflection nebulae in Monoceros, ApJ, 1975, vol. 199, p. 79

Kwok S., Physics and Chemistry of the Interstellar Medium, 2007 
Lada C. J., Star formation - From OB associations to protostars. In Star Forming Regions , vol. 115 of IAU Symposium, 1987, p. 1

Lasker B. M., Russell J. L., Jenkner H., Sturch C. R., McLean B. J., Shara M. M., The HST Guide Star Catalog, Version 1.1 (Lasker+ 1992), VizieR Online Data Catalog, 1996, vol. 1220 , p. 0

Li J. Z., Smith M. D., Discovery of Multiseeded Multimode Formation of Embedded Clusters in the Rosette Molecular Complex, ApJ, 2005, vol. 620, p. 816

López-Santiago J., Caballero J. A., New deep XMM-Newton observations to the west of the $\sigma$ Orionis cluster, A\&A, 2008, vol. 491, p. 961

Loren R. B., Peters W. L., Vanden Bout P. A., Collapsing molecular clouds, ApJ, 1974, vol. 194, p. L103

Lucy L. B., White R. L., X-ray emission from the winds of hot stars, ApJ, 1980, vol. 241, p. 300

Lépine J., A Via Láctea, Nossa Ilha no Universo. São Paulo: EDUSP, 2008, 288 p.

Lépine J. R. D., Duvert G., Star formation by infall of high velocity clouds on the galactic disk, A\&A, 1994, vol. 286, p. 60

Maaskant K. M., Bik A., Waters L. B. F. M., Kaper L., Henning T., Puga E., Horrobin M., Kainulainen J., Sequential star formation in IRAS 06084-0611 (GGD 12-15). From intermediate-mass to high-mass stars, A\&A, 2011, vol. 531, p. A27

Maciel J. W., Astrofísica do Meio Interestelar. São Paulo: EDUSP, 2002, 360 p.

Maddalena R. J., Morris M., Moscowitz J., Thaddeus P., The large system of molecular clouds in Orion and Monoceros, ApJ, 1986, vol. 303, p. 375

McKee C. F., Tan J. C., The Formation of Massive Stars from Turbulent Cores, ApJ, 2003, vol. 585 , p. 850

Miesch M. S., Scalo J., Bally J., Velocity Field Statistics in Star-forming Regions. I. Centroid Velocity Observations, ApJ, 1999, vol. 524, p. 895 
Minier V., Burton M. G., T. H., et al. Star-forming protoclusters associated with methanol masers, A\&A, 2005, vol. 429, p. 945

Mirabel I. F., Franco M., Estudio de una Pequeña Estructura del Hidrógeno Neutro con Alta Velocidad Expulsada del Núcleo de la Galaxia., Boletin de la Asociacion Argentina de Astronomia La Plata Argentina, 1981, vol. 20, p. 175

Monfredini T., Formação de água e emissão maser em IRAS 16293-2422, São José dos Campos: Instituto Nacional de Pesquisas Espaciais - INPE, 2009, Dissertação de Mestrado

Montmerle T., Koch-Miramond L., Falgarone E., Grindlay J. E., Einstein observations of the Rho Ophiuchi dark cloud - an X-ray Christmas tree, ApJ, 1983, vol. 269, p. 182

Motte F., Zavagno A., Bontemps S., Schneider N., Hennemann M., di Francesco J., André P., Saraceno P., Griffin M., Marston A., Ward-Thompson D., et al Initial highlights of the HOBYS key program, the Herschel imaging survey of OB young stellar objects, A\&A, 2010, vol. 518, p. L77

Nakajima H., Imanishi K., Takagi S.-I., Koyama K., Tsujimoto M., X-Ray Observation on the Monoceros R2 Star-Forming Region with the Chandra ACIS-I Array, PASJ, 2003, vol. 55 , p. 635

Olano C. A., Poeppel W. G. L., Kinematical origin of the dark clouds in Taurus and of some nearby galactic clusters, A\&A, 1987, vol. 179, p. 202

Parker N. D., Infrared properties of IRAS sources associated with nearby dark molecular clouds, MNRAS, 1991, vol. 251, p. 63

Parker R. J., Goodwin S. P., Do O-stars form in isolation?, MNRAS, 2007, vol. 380, p. 1271

Pereyra A., Girart J. M., Magalhães A. M., Rodrigues C. V., de Araújo F. X., Near infrared polarimetry of a sample of YSOs, A\&A, 2009, vol. 501, p. 595

Pereyra A., Magalhães A. M., Polarimetry toward the Musca Dark Cloud. I. The Catalog, ApJ, 2004, vol. 603, p. 584 
Pereyra A., Rodrigues C. V., Magalhães A. M., Polarimetry of the binary PDS 144, A\&A, 2012, vol. 538, p. A59

Pérez M. R., The young open clusters NGC 2244 and NGC 2264, revisited., Rev. Mexicana Astron. Astrofis., 1991, vol. 22, p. 99

Perkins F., Gold T., Salpeter E. E., Maser Action in Interstellar OH, ApJ, 1966, vol. 145, p. 361

Phelps R. L., Lada E. A., Spatial Distribution of Embedded Clusters in the Rosette Molecular Cloud: Implications for Cluster Formation, ApJ, 1997, vol. 477, p. 176

Poulton C. J., Robitaille T. P., Greaves J. S., Bonnell I. A., Williams J. P., Heyer M. H., A Spitzer survey of young stellar objects in the Rosette Molecular Cloud, MNRAS, 2008, vol. 384 , p. 1249

Preibisch T., Zinnecker H., The History of Low-Mass Star Formation in the Upper Scorpius OB Association, AJ, 1999, vol. 117, p. 2381

Price S., Cohen M., Walker R., Hackwell J., Henry R., Moshir M., Paxton L., Tedesco E., Walker R., Witteborn F., Astronomy With the Midcourse Space Experiment (MSX) Satellite. In American Astronomical Society Meeting Abstracts, vol. 26 of Bulletin of the American Astronomical Society, 1994, p. 1323

Reid M. J., Moran J. M., Masers, ARA\&A, 1981, vol. 19, p. 231

Richards P. J., Little L. T., Heaton B. D., Toriseva M., HCO(+) survey of unassociated compact molecular clouds in the IRAS Point Source Catalog, MNRAS, 1987, vol. 228, p. 43

Rodrigues C. V., Sartori M. J., Gregorio-Hetem J., Magalhães A. M., The Alignment of the Polarization of Herbig Ae/Be Stars with the Interstellar Magnetic Field , vol. 698, 2009, p. 2031

Román-Zúñiga C. G., Near infrared study of the star-forming properties of the Rosette Complex, University of Florida, 2006, Tese de Doutorado

Román-Zúñiga C. G., Lada E. A., , 2008 Star Formation in the Rosette Complex. p. 928 
Sartori M. J., Lépine J. R. D., Dias W. S., Formation scenarios for the young stellar associations between galactic longitudes $1=280 \mathrm{degr}-360 \mathrm{degr}$, A\&A, 2003, vol. 404, p. 913

Shuping R. Y., Kassis M., Morris M., Smith N., Bally J., Silicate Emission Profiles from Low-Mass Protostellar Disks in the Orion Nebula: Evidence for Growth and Thermal Processing of Grains, ApJ, 2006, vol. 644, p. L71

Skrutskie M. F., Cutri R. M., Stiening et al. The Two Micron All Sky Survey (2MASS), AJ, 2006, vol. 131, p. 1163

Stahler S. W., Shu F. H., Taam R. E., The evolution of protostars. II - The hydrostatic core, ApJ, 1980

Sunada K., Nakazato T., Ikeda N., Hongo S., Kitamura Y., Yang J., Water Maser and Ammonia Survey toward IRAS Sources in the Galaxy I. $\mathrm{H}_{2} \mathrm{O}$ Maser Data, PASJ, 2007, vol. 59 , p. 1185

Tafalla M., Bachiller R., Wright M. C. H., Dense clumps in the Monoceros R2 outflow, ApJ, 1994, vol. 432, p. L127

Tielens A. G. G. M., The Physics and Chemistry of the Interestellar Medium. United Kingdom: Cambridge University Press, 2005, 495 p.

Townsley L. K., Feigelson E. D., Montmerle T., Broos P. S., Chu Y.-H., Garmire G. P., 10 MK Gas in M17 and the Rosette Nebula: X-Ray Flows in Galactic H II Regions, ApJ, 2003, vol. 593, p. 874

Wang J., Feigelson E. D., Townsley L. K., Román-Zúñiga C. G., Lada E., Garmire G., A Chandra Study of the Rosette Star-Forming Complex. II. Clusters in the Rosette Molecular Cloud, ApJ, 2009, vol. 696, p. 47

Wang J., Townsley L. K., Feigelson E. D., Broos P. S., Getman K. V., Román-Zúñiga C. G., Lada E., A Chandra Study of the Rosette Star-forming Complex. I. The Stellar Population and Structure of the Young Open Cluster NGC 2244, ApJ, 2008, vol. 675, p. 464 
Ward-Thompson D., Di Francesco J., Hatchell J., Hogerheijde M. R., Nutter D., Bastien P., Wouterloot J., Yates J., Zhu M., The James Clerk Maxwell Telescope Legacy Survey of Nearby Star-forming Regions in the Gould Belt, PASP, 2007, vol. 119, p. 855

Williams J. P., Blitz L., Stark A. A., The Density Structure in the Rosette Molecular Cloud: Signposts of Evolution, ApJ, 1995, vol. 451, p. 252

Wood D. O. S., Churchwell E., The morphologies and physical properties of ultracompact H II regions, ApJS, 1989, vol. 69, p. 831

Xie T., Shells, outflows and star formation in the giant molecular cloud Monoceros R2, Massachusetts Univ., Boston., 1992, Tese de Doutorado

Xie T., Goldsmith P. F., The giant molecular cloud Monoceros R2. 1: Shell structure, ApJ, 1994, vol. 430, p. 252

Zinnecker H., Yorke H. W., Toward Understanding Massive Star Formation, ARA\&A, 2007, vol. 45, p. 481 
Apêndice 

Apêndice A

\section{Masers}

Linhas de emissão de transições moleculares são observadas em regiões de formação estelar. Uma emissão, em particular, é chamada de maser. O termo maser vem da sigla em inglês para "Microwave Amplification by Stimulated Emission of Radiation", ou seja, "Amplificação de Microondas por Emissão Estimulada de Radiação". O processo que produz o maser é análoga ao laser, mas ocorre para frequências compreendidas na faixa rádio - nesse caso, as microondas (Elitzur, 1992). Simplificadamente, considerando um sistema de dois níveis para a transição (nível superior e nível inferior), três processos podem ocorrer: emissão espontânea, absorção e emissão estimulada. O maser pode ser explicado através de um sistema de três níveis de energia, mas ocorrem os mesmos tipos de processo.

A emissão maser tem maior probabilidade de ocorrer quando um número maior de moléculas está em um estado excitado de energia em relação às moléculas que se encontram em um estado de energia inferior. Mas como a população de um determinado nível tende a diminuir com a energia do nível, ocorre o processo conhecido por "inversão de população" (Lépine, 2008).

A Figura A.1 ilustra um esquema de três níveis de energia de moléculas que emitem maser. A transição do nível 1 (de menor energia) para o nível 3 (de maior energia) é causada por um bombeamento. A população do nível 3 terá uma transição espontânea para o nível 2, o que resultará em uma população anômala, pois a transição do nível 2 para o nível 1 é pouco provável. Apenas quando a radiação estimular a população no nível 2, ocorrerá outra transição com emissão de radiação. Portanto, a emissão maser é resultado da amplificação da radiação conforme esta atravessa a região onde ocorre a inversão de 
população.

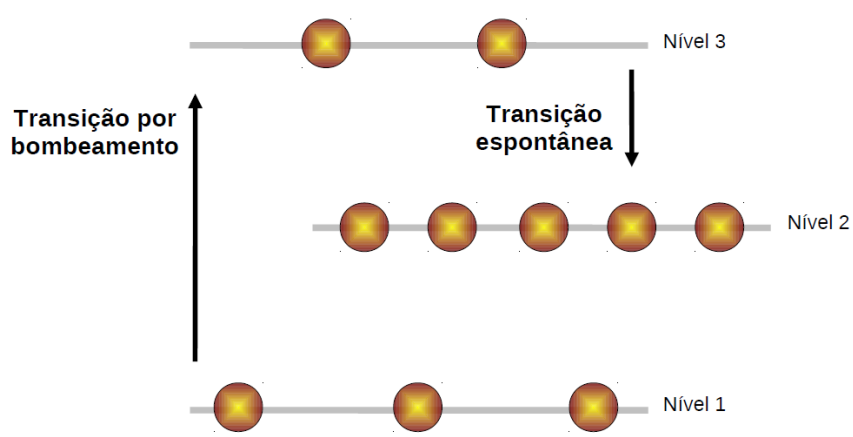

Figura A.1: Ilustração das transições que ocorrem na emissão maser, na qual os círculos representam a população de moléculas que estão presentes nos níveis de energia. Adaptado de Lépine (2008).

A energia emitida pela transição do nível 2 para o nível 1 é somada à energia da radiação incidente, que percorre a mesma direção. A causa da inversão de população seria algum tipo de "bombeamento" de natureza radiativa ou colisional (Maciel, 2002). Dessa maneira, choques dissociativos provindos de estrelas quentes e massivas associados a fragmentos de nuvens moleculares parecem fornecer o ambiente adequado para explicar a observação de masers de água nessas regiões HII (Elitzur, 1992).

As observações têm mostrado que as condições físicas necessárias para a produção da excitação maser de água excedem densidades de $10^{7} \mathrm{~cm}^{-3}$ e ocorrem a baixas temperaturas ( $100 \mathrm{~K})$, como indica o trabalho de Breen et al. (2007). Essas características são vistas em discos circunstelares ao redor de objetos estelares jovens e em regiões de gás que sofrem choques, como sugerem Reid e Moran (1981).

Durante décadas vários estudos vem sendo realizados, com o objetivo de detectar essa amplificação de radiação na direção de regiões de formação estelar.

O maser geralmente se encontra associado ao gás aquecido devido às ondas de choque em regiões HII ou em envoltórios de estrelas nas fases mais primitivas de sua formação. A emissão maser desempenha um papel importante no entendimento dos processos envolvidos na formação de estrelas massivas, pois através dessas intensas linhas de emissão é possível obter informações das condições físicas e dinâmicas do meio interestelar (Reid e Moran, 1981). De fato, a emissão maser é observada no envoltório molecular de estrelas massivas 
em seus estágios iniciais da formação (Breen et al., 2010) e podem ser observados nessa fase de alto embebimento porque são detectados na faixa de radiofrequência.

Perkins, Gold e Salpeter (1966) foram os primeiros a propor a amplificação maser para interpretar a intensa emissão da hidroxila $(\mathrm{OH})$ detectada na direção de regiões HII. A Figura A.2 representa condição física teorizada pelos autores.
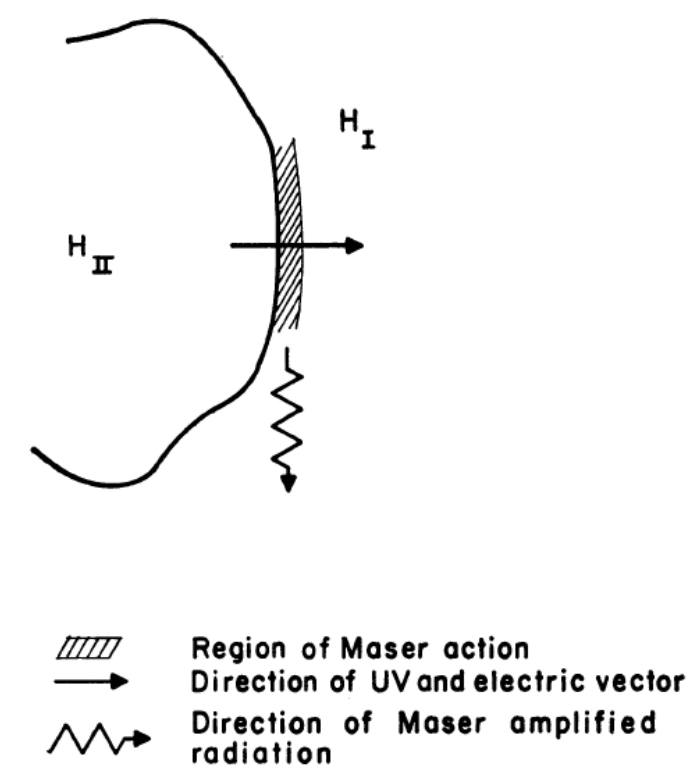

Figura A.2: Geometria sugerida por Perkins, Gold e Salpeter (1966), na qual a ocorrência de maser seria esperada.

A água $\left(\mathrm{H}_{2} \mathrm{O}\right)$ foi a segunda espécie molecular detectada como maser em regiões de formação estelar ativa (Cheung et al., 1969). Posteriormente, outras moléculas como monóxido de silício $(\mathrm{SiO})$ e metanol $\left(\mathrm{CH}_{3} \mathrm{OH}\right)$ foram descobertas na região da nebulosa de Orion.

O processo maser tem sua linha de emissão caracterizada pela alta intensidade e regiões muito compactas. Ela ocorre em regiões nas quais a densidade do gás está entre $10^{5}$ e $10^{11}$ $\mathrm{cm}^{-3}$ (Reid e Moran, 1981), uma densidade maior do que aquela tipicamente encontrada nas nuvens moleculares gigantes $\left(10^{3}\right.$ a $\left.10^{5} \mathrm{~cm}^{-3}\right)$. Emissão maser interestelar, resultante da transição de moléculas de água, hidroxila e metanol, é detectada na direção de diversas regiões de formação estelar na nossa Galáxia (Breen et al., 2010).

Estrelas $\mathrm{O}$ e B, em formação ou recentemente formadas, aquecem e ionizam o ambiente que as abrigam, criando fontes que possuem emissão no infravermelho e em rádio. 
Para os masers associados com tais regiões de formação estelar, essas estrelas quentes e massivas fornecem a energia necessária para que o maser seja produzido. Observa-se que as moléculas que produzem emissão maser estão contidas em condensações de matéria interestelar na proximidade desses objetos jovens e tais masers parecem estar associados às regiões HII (Minier et al., 2005). Como será visto, alguns masers estão relacionados com regiões de choque. Assim, a presença de maser pode traçar a interação entre os ventos de alta velocidade, originados pelas estrelas OB que se encontram no centro da região HII, e o gás denso que a circunda.

A transição rotacional $6_{1,6} \rightarrow 5_{2,3}$ da água, que ocorre em $22 \mathrm{GHz}$, é uma linha espectral muito intensa em comprimentos de onda rádio $(\lambda \simeq 1.3 \mathrm{~cm})$. Essa linha é interessante, em particular, pois traça o gás que está sofrendo choque devido ao vento provindo das estrelas em formação (Breen et al., 2007).

As altas velocidades de certas linhas observadas indicam que os masers estão longe das condensações centrais da região (Elitzur, 1992). Eles se encontram associados a estágios evolutivos nos quais fortes fluxos de material estão presentes. O perfil de linha do espectro de um maser de água será tratado mais adiante.

Breen et al. (2010) observaram emissão rádio na direção de regiões de formação estelar e detectaram masers de hidroxila, água e metanol. Dentro de tais regiões, os masers mostraram se aglomerar em pequenos grupos com diferentes combinações de espécies moleculares, o que é um indicativo de estrelas jovens massivas em diferentes estágios de evolução. 
Apêndice B

\section{Busca de Fontes Maser no ROI}

O Apêndice B destina-se a descrever as técnicas utilizadas nas observações realizadas no Rádio Observatório do Itapetinga (ROI), o tratamento dos espectros obtidos e a seleção de amostras para possível detecção maser. Como referência, nos baseamos em trabalhos como Monfredini (2009), que estudou a emissão maser de água na direção da fonte IRAS 162932422, e Almeida (2006), que estudou o complexo de regiões UC HII RCW 95, mapeando a nuvem no contínuo em $43 \mathrm{GHz}$ e detectando emissão maser nas três fontes IRAS presentes no complexo.

\section{B.1 O rádio telescópio}

O ROI, localizado no município de Atibaia, São Paulo, abriga uma antena de 13.7 metros de diâmetro. Esse rádiotelescópio é adequado para utilizar um receptor que opera na banda de $22 \mathrm{GHz}$, operando na frequência da transição do maser de $\mathrm{H}_{2} \mathrm{O}$.

\section{B.1.1 Rastreio}

O programa de rastreio permite que os parâmetros sejam adequados ao tipo de observação que se pretende realizar. Nesse caso, para observação de linha em $22 \mathrm{GHz}$ (detecção da emissão maser da molécula $\mathrm{H}_{2} \mathrm{O}$ ) foi utilizado uma varredura do tipo On-Off, que consiste em fazer uma varredura na "fonte" e outra no "céu". Esse procedimento permite subtrair a contribuição do "céu" e diminuir o ruído na observação. A integração sobre cada fonte pode ser descrita por uma série de 10. Cada ciclo consiste em captar emissão durante 20 segundos sobre a fonte (que é o tempo de duração da varredura em Azimute). 


\section{B.1.2 Aquisição}

O programa de aquisição de dados ajusta o sintetizador de frequência para a molécula selecionada e permite a calibração em linhas e em velocidade. Depois de realizar a observação das fontes, os dados de saída do programa de aquisição do rádio observatório gera arquivos de texto, no formato *.txt. Porém, para serem interpretados, esses dados precisam ser reduzidos em um software chamado DRAWSPEC. O passo primário é converter os arquivos *.txt para arquivos *.DAT, que é a extensão utilizada pelo programa para gerar os espectros desejados.

\section{B.1.3 Redução dos dados}

A redução de dados pode ser resumida em 5 etapas. A primeira etapa é obter o espectro de cada uma das integrações. No exemplo a seguir, é utilizada uma fonte que apresenta uma linha em emissão. Cada espectro precisa ser avaliado para que nenhuma contaminação ocorra no resultado final da redução. As Figuras B.1, B.2, B.3 e B.4 apresentam espectros da mesma fonte, obtidos na data de 12/10/2011.

A segunda etapa é realizar a soma dos espectros, de modo a se obter um maior tempo de integração sobre a fonte, para atingir uma razão sinal/ruído melhor. O resultado dessa soma está na Figura B.5.

Na sequência, a linha de base do espectro é subtraída. Para isso, são escolhidos dois boxes, um de cada lado da linha em emissão (Figura B.6), para que a linha de base seja calculada e subtraída (Figura B.7). Alguns parâmetros podem ser modificados no header do espectro.

A etapa subsequente é separar o espectro em partes, de forma que menores detalhes possam ser visualizados (Figura B.8). Além disso, fotodiodos centrais são mais sensíveis à radiação, logo as informações laterais são "distorcidas" e podem ser descartadas nessa situação, onde não há características relevantes. Após selecionar a parte de interesse, o procedimento padrão é fazer uma média entre fotodiodos vizinhos (Figura B.9), resultando em uma suavização do sinal, para que nenhuma discrepância altere o resultado final de forma indevida.

Influências ondulatórias ocasionadas pelo caminho da radiação na própria antena e interação com a redoma podem resultar em uma modulação no espectro, que não é da 


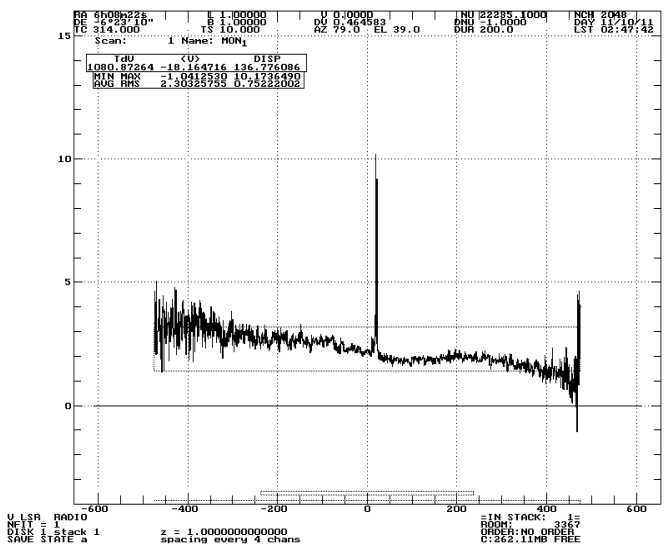

Figura B.1: Espectro 1 de Mon 1 (IRAS 06053-0622). Tempo de integração $=200$ s.

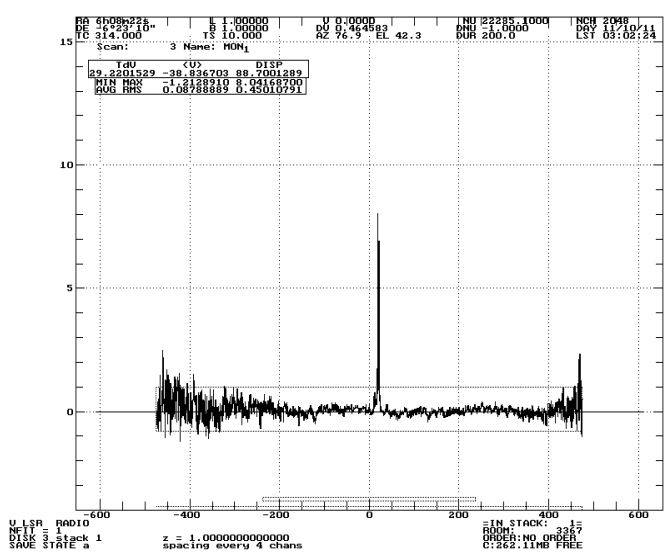

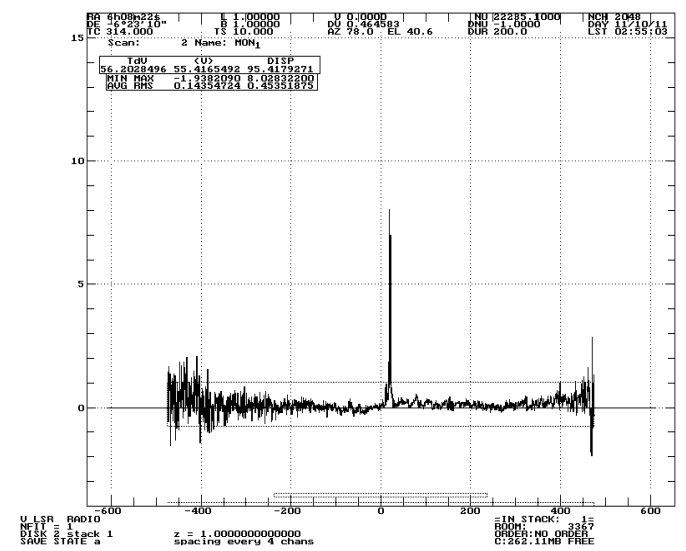

Figura B.2: Espectro 2 de Mon 1. Tempo de integração $=200 \mathrm{~s}$.

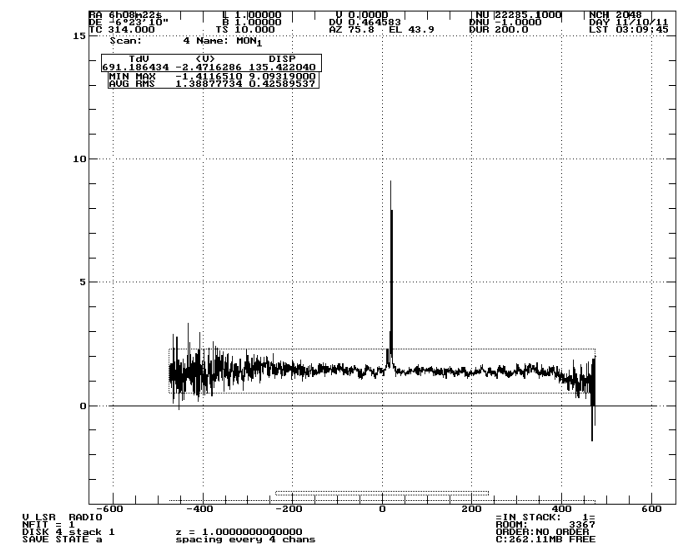

Figura B.3: Espectro 3 de Mon 1. Tempo de integração $=200 \mathrm{~s}$.

Figura B.4: Espectro 4 de Mon 1. Tempo de integração $=200 \mathrm{~s}$.

natureza da fonte. Para retirá-la, ajusta-se uma onda estacionária com amplitude regulável, que é subtraída em seguida (Figura B.10).

Após essas etapas de redução, o espectro pode ser considerado adequado para análise e qualquer ajuste pode ser feito após esses passos, de acordo com a necessidade do usuário ou a peculiaridade dos dados que estão sendo analisados. 


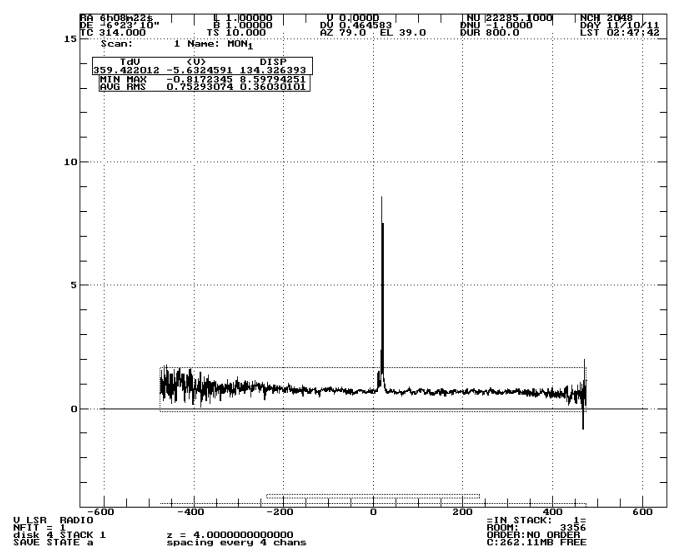

Figura B.5: Espectros 1, 2, 3 e 4 de Mon 1, somados. Tempo total $=800 \mathrm{~s}$.

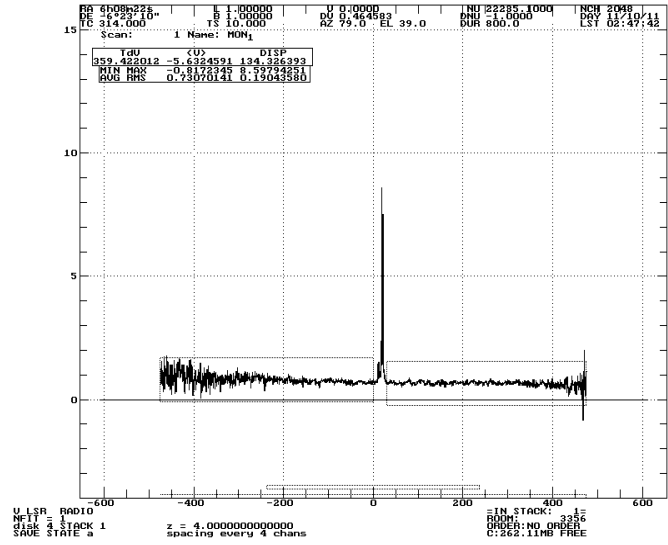

Figura B.6: Selecionando os dois boxes para extrair a linha de base.

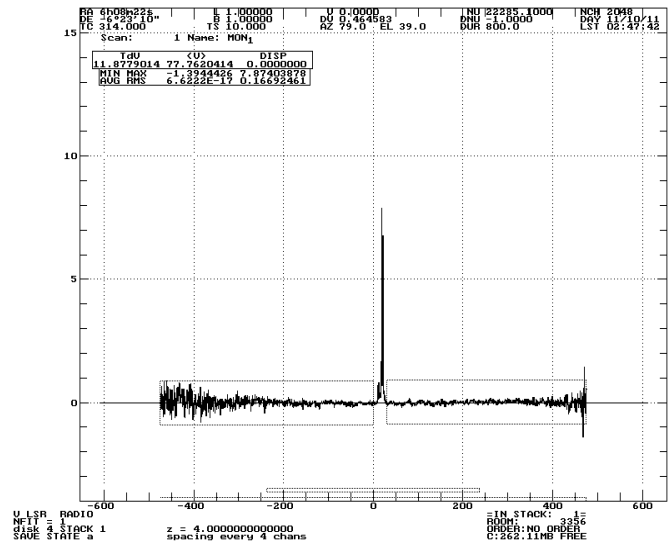

Figura B.7: Linha de base extraída e parâmetros alterados.

\section{B.2 Observações}

As observações de candidatas a fontes maser ocorreu em diferentes épocas entre Maio de 2010 e Dezembro de 2011. Para cada região de interesse foi selecionada uma lista de alvos. Os critérios de seleção da amostra e as características dos alvos serão descritas mais adiante.

\section{B.2.1 Mon R2}

A busca por fontes maser na nuvem Mon R2 baseou-se em uma amostra de fontes a serem observadas ROI, em Atibaia. O critério de seleção de fontes foi estabelecido da 


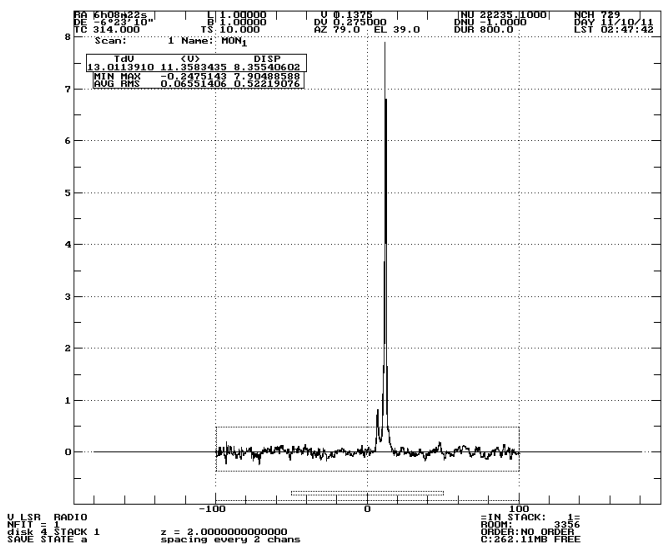

Figura B.8: Espectro após os cortes laterais.

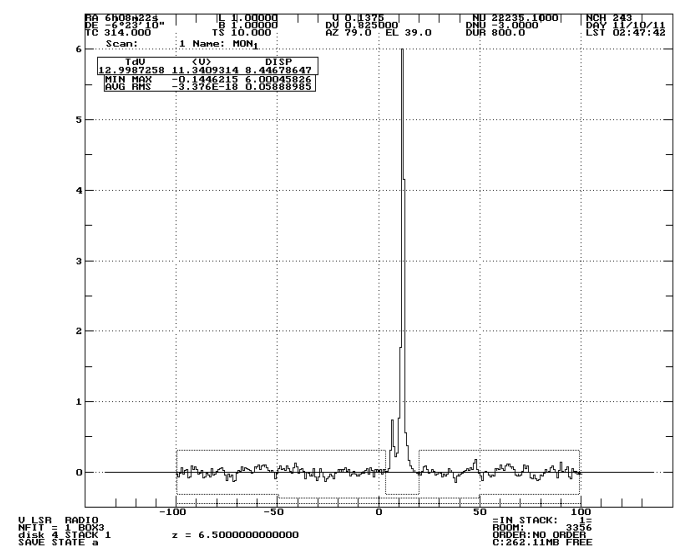

Figura B.9: Após o cálculo da média entre os fotodiodos, o espectro aparece suavizado.

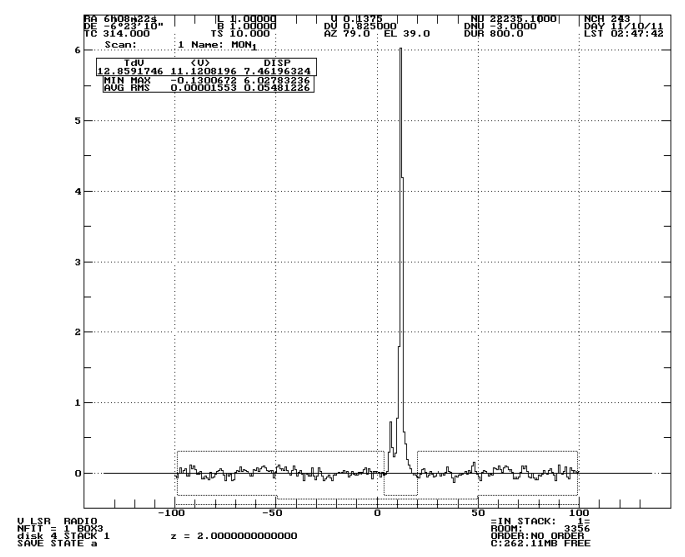

Figura B.10: Onda estacionária removida do espectro.

seguinte forma:

- objetos que apresentam características típicas de associação com regiões de formação estelar: alta emissão em $100 \mu \mathrm{m}$ (fontes IRAS), fontes 2MASS associadas embebidas, fontes de raios-X identificadas como estrela pré-sequência principal de massa intermediária (Gregorio-Hetem et al., 1998) e fontes maser anteriormente detectadas nessa nuvem por Codella et al. (1995); Sunada et al. (2007).

- objetos jovens nas proximidades de estrelas do tipo B (Herbst e Racine, 1976). 
A amostra foi então composta através da seleção de 26 candidatas, das quais: $57 \%$ são fontes 2MASS, $69 \%$ são fontes IRAS, $26 \%$ são fontes de raios-X e $38 \%$ são fontes maser (com confirmação prévia).

As observações consistiram em detectar a transição de maser de água utilizando o receptor de raia de $22 \mathrm{GHz}$, que equivale ao comprimento de onda $\lambda=1.3 \mathrm{~cm}$. A antena de 14 metros do ROI foi utilizada em dois períodos do ano, Maio e Agosto de 2011, para a observação desses objetos. Os períodos correspondem ao tempo concedido para esse projeto, que conta com a colaboração do Prof. José Williams Villas-Boas (INPE/MCT) e Thiago Monfredini (Valongo/UFRJ). Realizamos integrações entre 200 e 2800 segundos por alvo, buscando valores aceitáveis de ruído (minimizando o $\mathrm{rms}$ - root mean square) e procurando obter boa razão sinal/ruído nas observações. A Tabela B.1 traz informações sobre os alvos selecionados para as observações. A primeira coluna mostra a identificação, as segunda e terceira colunas mostram as coordenadas equatoriais dos objetos (obtidas através do banco de dados astronômicos SIMBAD) e as colunas seguintes trazem a identificação $2 M A S S$, IRAS e ROSAT de cada candidato a maser, quando disponível.

A primeira coluna mostra uma identificação proposta por nós, na qual os objetos são enumerados de acordo com a prioridade para observação. Os alvos prioritários são fontes IRAS brilhantes (principais candidatos a YSOs na região); em seguida observamos fontes IRAS associadas com masers de água já antes identificados (buscando possíveis variações em intensidade, já que masers astrofísicos são altamente variáveis no tempo) e, na sequência de prioridade dos alvos, estão as fontes de raios-X com contrapartida 2MASS e que se localizam na região de interesse. A inclusão das fontes Mon_17, Mon_18 e Mon_19 foi baseada na alta magnitude na banda $\mathrm{K}$ que essas fontes IRAS apresentam, na faixa de 12 a 15 mag.

Como resultado das observações, a intensidade da emissão é medida através de um ajuste do perfil da linha observada, que em geral é fornecido em termos de temperatura de antena ( $\mathrm{T}_{A}$, que é proporcional a temperatura de brilho da fonte maser) em função da velocidade radial em relação ao LSR (Local Standard of Rest).

Esses perfis de linha, ou espectro, são então reduzidos com o programa DRAWSPEC e os resultados são exemplificados nas figuras a seguir. A partir de uma pré-análise, destacamos dois exemplos: a Figura B.11 ilustra um resultado no qual não encontramos 
Tabela B.1 - Lista de alvos em Mon R2, para observação em rádiofrequência.

\begin{tabular}{|c|c|c|c|c|c|}
\hline ID & $\begin{array}{c}\operatorname{AR}(2000) \\
(\mathrm{hms})\end{array}$ & $\begin{array}{c}\operatorname{Dec}(2000) \\
\left({ }^{\circ}, "\right)\end{array}$ & 2MASS & IRAS & RXJ \\
\hline Mon_1 & $06: 07: 46.7$ & $-06: 23: 00$ & $\ldots$ & 06053-0622 & $\ldots$ \\
\hline Mon_2 & $06: 07: 27.5$ & $-06: 23: 47$ & 06072549-0623487 & 06050-0623 & $\ldots$ \\
\hline Mon_3 & $06: 10: 51.0$ & $-06: 11: 54$ & $\ldots$ & 06084-0611 & $\ldots$ \\
\hline Mon_4 & $06: 09: 30.0$ & $-06: 19: 40$ & $\ldots$ & 06070-0619 & $\ldots$ \\
\hline Mon_5 & $06: 12: 48.3$ & $-06: 13: 19$ & 06125036-0613114 & 06103-0612 & $\ldots$ \\
\hline Mon_6 & $06: 08: 29.5$ & $-06: 18: 26$ & $\ldots$ & 06060-0617 & $\ldots$ \\
\hline Mon_7 & $06: 07: 06.7$ & $-05: 37: 24$ & $\ldots$ & 06046-0603 & $\ldots$ \\
\hline Mon_8 & $06: 07: 43.5$ & $-05: 34: 17$ & $\ldots$ & $06052-0533$ & $\ldots$ \\
\hline Mon_9 & $06: 07: 31.6$ & $-05: 10: 21$ & $\ldots$ & 06050-0509 & $\ldots$ \\
\hline Mon_10 & $06: 05: 08.2$ & $-07: 14: 42$ & $\ldots$ & 06027-0714 & $\ldots$ \\
\hline Mon_11 & $06: 07: 52.7$ & $-05: 16: 04$ & 06075243-0516036 & 06054-0515 & $\ldots$ \\
\hline Mon_12 & $06: 07: 45.0$ & $-05: 12: 40$ & $\ldots$ & 06052-0512 & $\ldots$ \\
\hline Mon_13 & $06: 08: 04.2$ & $-07: 00: 38$ & $\ldots$ & 06056-0700 & $\ldots$ \\
\hline Mon_14 & $06: 13: 36.2$ & $-06: 25: 01$ & 06133726-0625017 & 06111-0624 & $\ldots$ \\
\hline Mon_15 & $06: 08: 23.6$ & $-06: 33: 02$ & 06082356-0632552 & 06059-0632 & $\ldots$ \\
\hline Mon_16 & $06: 06: 58.5$ & $-05: 55: 08$ & 06065848-0555066 & 06045-0554 & $\ldots$ \\
\hline Mon_17 & $06: 07: 19.5$ & $-06: 17: 40$ & $06071952-0617405$ & $\ldots$ & $\ldots$ \\
\hline Mon_18 & $06: 07: 20: 3$ & $-06: 17: 33$ & 06072037-0617339 & $\ldots$ & $\ldots$ \\
\hline Mon_19 & $06: 07: 21.5$ & $-06: 17: 41$ & 06072156-0617418 & $\cdots$ & $\ldots$ \\
\hline Mon_20 & $06: 07: 18.7$ & $-06: 23: 41$ & 06071830-0623440 & $\ldots$ & 0607.3-0623 \\
\hline Mon_21 & $06: 07: 41.1$ & $-06: 26: 41$ & 06074057-0626554 & $\ldots$ & 0607.6-0626 \\
\hline Mon_22 & $06: 07: 50.9$ & $-06: 25: 51$ & 06075071-0625529 & $\cdots$ & 0607.8-0625 \\
\hline Mon_23 & $06: 07: 55.1$ & $-06: 16: 53$ & 06075493-0616475 & $\ldots$ & 0607.9-0616 \\
\hline Mon_24 & $06: 08: 48.2$ & $-06: 49: 32$ & $\ldots$ & 06063-0648 & 0608.7-0649 \\
\hline Mon_25 & $06: 08: 42.4$ & $-06: 38: 23$ & 06084191-0638193 & $\ldots$ & 0608.7-0638 \\
\hline Mon_26 & $06: 09: 34.7$ & $-05: 44: 06$ & 06093206-0543027 & 06070-0542 & 0609.5-0544 \\
\hline
\end{tabular}


uma linha em emissão clara, pois a razão sinal/ruído é baixa; a Figura B.12 traz um resultado positivo, no qual a linha é observada na velocidade esperada (aproximadamente na velocidade da nuvem) e o rms da obsevação permite uma razão sinal/ruído muito boa para uma análise mais detalhada no futuro. Os dados de todos os espectros obtidos para os alvos da amostra ainda encontram-se em fase de análise.

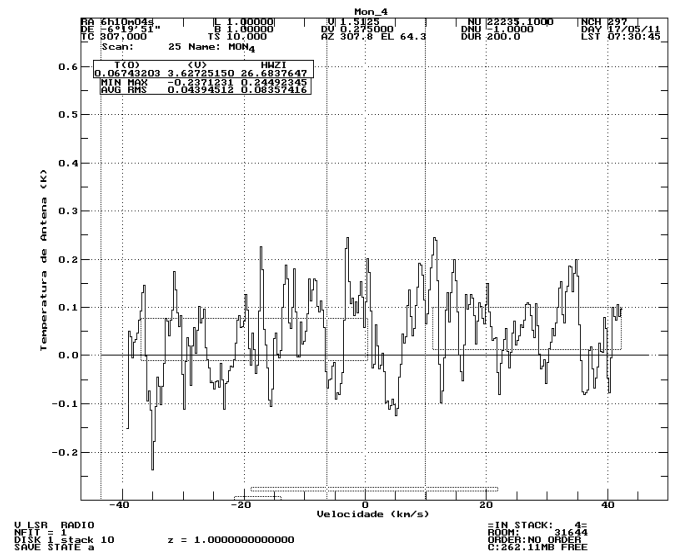

Figura B.11: Espectro da fonte IRAS 06070-0619 (Mon_4), obtido por meio de nossas observações e não apresenta linhas de emissão.

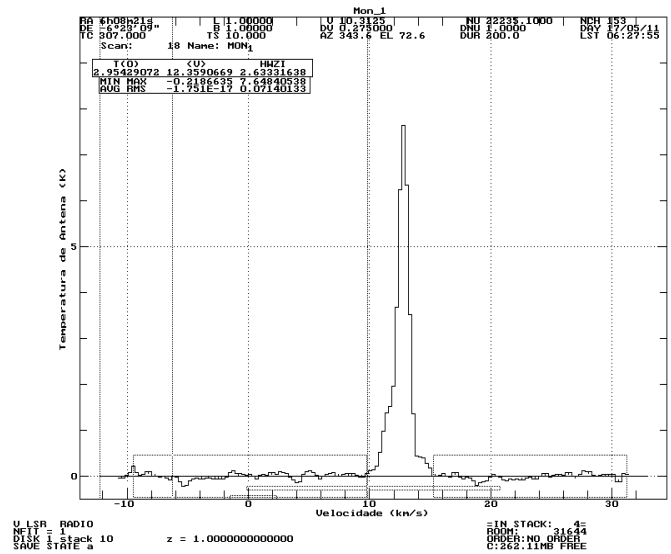

Figura B.12: Espectro da fonte 060530622 (Mon_1), apresentando linha em emissão maser de $\mathrm{H}_{2} \mathrm{O}$ bastante intensa.

\section{B.2.2 RMC}

A amostra selecionada para a busca de fontes maser em $R M C$ teve como ponto inicial os 70 candidatos a objetos pré-estelares de Williams et al. (1995), para os quais foi realizada uma inspeção de imagens ópticas e no infravermelho. Foram associados aos clumps fontes pontuais IRAS e fontes de raios-X detectadas pelo ROSAT. A amostra, composta por 9 fontes, é descritas na Tabela B.2.

A dificuldade em se detectar emissão maser, especificamente nessa região, reside na distância de $R M C$. O rádiotelescópio deveria oferecer maior sensibilidade ou o tempo de integração deveria ser maior, pos a emissão das candidatas pode estar abaixo do limite de detecção. As Figuras B.13 e B.14 ilustram dois exemplos de espectros obtidos para duas das fontes listadas na Tabela B.2. 
Tabela B.2 - Idem à Tabela B.1, para $R M C$.

\begin{tabular}{ccccc}
\hline \hline ID & $\begin{array}{c}\text { AR(2000) } \\
(\mathrm{hms})\end{array}$ & $\begin{array}{c}\operatorname{Dec}(2000) \\
\left({ }^{\circ}, "\right)\end{array}$ & IRAS & RXJ \\
\hline 1_IRAS & $06: 34: 32.8$ & $04: 13: 07$ & $06319+0415$ & $\ldots$ \\
4_IRAS & $06: 34: 04.9$ & $04: 25: 00$ & $06314+0427$ & $\ldots$ \\
13_IRAS & $06: 35: 32.3$ & $03: 58: 52$ & $06329+0401$ & $\ldots$ \\
9_ROSAT & $06: 34: 03.3$ & $04: 19: 40$ & $\ldots$ & $0634.0+0419$ \\
2_IRAS & $06: 33: 31.1$ & $04: 00: 07$ & $06308+0402$ & $\ldots$ \\
3_IRAS & $06: 31: 48.5$ & $04: 19: 30$ & $06291+0421$ & $\ldots$ \\
5_IRAS & $06: 33: 16.2$ & $04: 34: 53$ & $06306+0437$ & $\ldots$ \\
10_IRAS & $06: 34: 53.0$ & $03: 47: 06$ & $06322+0349$ & $\ldots$ \\
11_IRAS & $06: 31: 58.0$ & $04: 01: 35$ & $06293+0403$ & $\ldots$ \\
15_ROSAT & $06: 34: 49.8$ & $04: 00: 45$ & $\ldots$ & $0634.8+0400$ \\
\hline
\end{tabular}

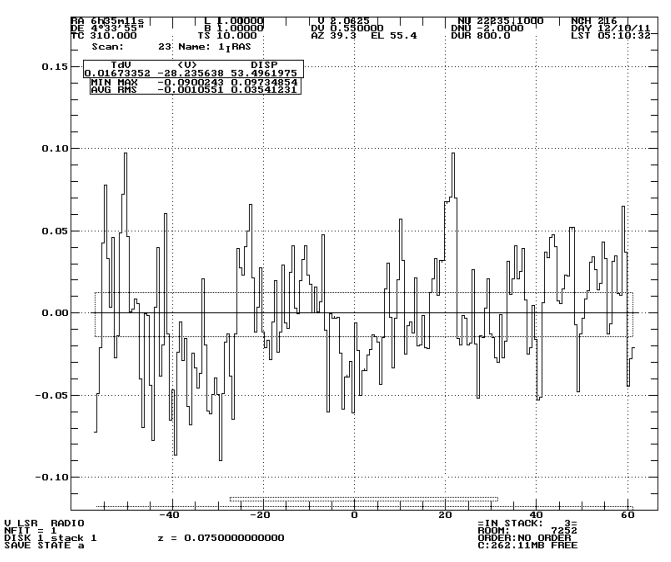

Figura B.13: Espectro da fonte IRAS 06319+0415 (1_IRAS), localizada em $R M C$.

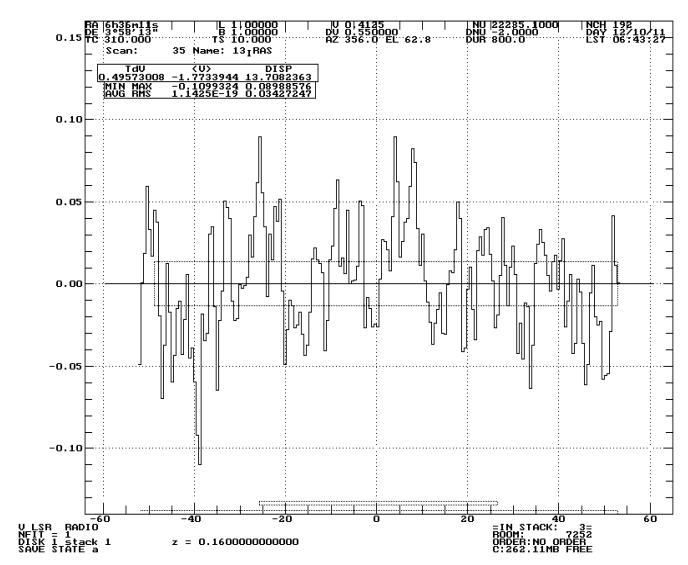

Figura B.14: Espectro da fonte IRAS 06329+0401 (13_IRAS), localizada em $R M C$. 